\title{
ORGANIC-INORGANIC HYBRID CATALYSTS FOR CHEMICAL PROCESSES OF INDUSTRIAL INTEREST
}

\author{
Doctoral Thesis
}

\author{
Candidate \\ ANDREA ERIGONI
}

Supervisor

Urbano Díaz Morales

Valencia, November 2020 



\section{Abstract}

\section{Resumen}

Resum 



\begin{abstract}
The research work described in this Doctoral Thesis was developed within the frame of the MULTI2HYCAT European Project (grant agreement N. 720783) and it is focused on the synthesis and characterization of mono- and multi-functional hybrid catalysts featuring acid, base or redox active sites. Several research lines have been developed in parallel to design multiple hybrid catalysts for different catalytic processes, building upon the needs of the industrial partners.

Due to the collaborative nature of the project, each academic partners mainly focused on one aspect of the whole process. Instituto de Tecnología Química (ITQ-CSIC), where this Thesis was developed, mostly focused on the design and synthesis of the hybrid catalysts. For that, part of the characterization results reported in Chapter 3 have been carried out at Università del Piemonte Orientale (IT), during a one month stay. Some of the catalytic results reported in Chapter 3 and Chapter 5 have been carried out by the University of Southampton (UK).
\end{abstract}

In Chapter 3 the synthesis of two different heterogeneous hybrid catalysts carrying arylsulfonic moieties, in which the aromatic ring was either fluorinated or not, is reported. Two multi-step synthetic approaches were developed, involving the synthesis of the silyl-derivative precursor, template-free one-pot co-condensation (at low temperature and neutral $\mathrm{pH}$ ) and tethering reaction. A multi-technique approach was implemented to characterize the hybrid catalysts. Textural properties, thermal stability and chemical makeup of the materials were studied. Moreover, probe molecules were adsorbed onto the hybrids and the interaction were studied with multi-nuclear NMR and FTIR spectroscopies. The catalytic activity of the two hybrids showed superior performances for the fluoro-aryl-sulfonic acid, compared to the non-fluorinated material, in the acetal formation between benzaldehyde and ethylene glycol. Silanol-capped versions of the hybrids have also been prepared and their properties have been compared with those of hydrophilic hybrids, to study the effect of the polarity of the surface on the overall catalytic activity of the hybrids.

In Chapter 4, the synthesis of hybrid mesoporous organocatalysts, obtained by grafting of commercial and custom-made silyl-derivatives onto MCM-41 supports, is reported. The hybrid catalysts were characterized and tested for different reactions involving C-C bond formation, such as Knoevenagel condensations and Michael addition. Finally, the catalysts were tested in the condensation between furfural and methyl isobutyl ketone and the most performing catalyst was selected for the synthesis of a multi-functional hybrid. Reaction mechanisms have been proposed and the beneficial effect of the surface silanol groups on the catalytic activity was demonstrated.

In Chapter 5, the synthesis of hybrid multi-functional catalysts is reported. Building upon the results reported in Chapter 4, a hybrid catalyst featuring aminopropyl moieties 
and palladium nanoparticles was developed. Structural and textural properties of the catalysts were accessed. Moreover, transmission electron microscopy showed a narrow nanoparticles distribution, centered a value equivalent to the size of the mesoporous channels of the support. The catalyst was tested in a tandem process involving the aldol condensation between furfural and methyl isobutyl ketone followed by hydrogenation of the aldol adduct. The influence of several variables on the activity of the multi-functional catalyst was explored, with the scope of paving the way for more thorough studies to be carried out in flow regime. Lastly, proof-of-concept syntheses of multi-functional hybrid catalysts featuring base sites and supported metal complex are reported. 


\section{Resumen}

El trabajo de investigación descrito en la presente Tesis Doctoral ha sido desarrollado en el marco del proyecto europeo MULTI2HYCAT (grant agreement N. 720783) y se ha centrado en la síntesis y caracterización de materiales mono- y multi-funcionales que presentan sitios catalíticos ácidos, básicos o redox. Diferentes líneas de investigación han sido desarrolladas en paralelo para obtener distintos materiales híbridos que serán empleados en diferentes procesos catalíticos, en línea con las necesidades de los socios industriales del proyecto.

Debido a la naturaleza colaborativa del proyecto, cada miembro académico se ha encentrado en un aspecto del desarrollo de los materiales. Es por ello que el Instituto de Tecnología Química (ITQ-CSIC), donde se ha llevado a cabo esta Tesis Doctoral, se ha centrado en la síntesis de los catalizadores híbridos. Por ello, parte de la caracterización descrita en el Capítulo 3 se ha llevado a cabo en la Università del Piemonte Orientale (IT), durante una estancia de un mes. Ademas, algúnos resultados catalíticos descritos en los Capítulos 3 y 5 han sido obtenidos por la University of Southampton (UK).

En el Capítulo 3, se ha descrito la síntesis de dos catalizadores heterogéneos híbridos que presentan moléculas de ácido aril-sulfónico en su composición. En uno de ellos, el anillo aromático presentará átomos de flúor en posición $2,3,5,6$. Se han llevado a cabo dos estrategias de síntesis multi-etapas, a través de la síntesis de los precursores alkoxisilanos, a través de procesos de condensación junto a un precursor de sílice (en ausencia de agentes directores de estructura, a $\mathrm{pH}$ neutro y temperaturas bajas) y de una reacción de tethering. Los materiales híbridos han sido caracterizados a través de diferentes técnicas. Las propiedades texturales, la estabilidad térmica y la composición química de los catalizadores ha sido estudiada. Además, moléculas sondas han sido adsorbidas en los materiales hibridos y las interacciones entre ellos han sido estudiadas a través de espectroscopias FTIR y RMN multi-nuclear. El catalizador hibrido en que el anillo aromático estaba fluorado resultó ser el más activo catalíticamente en la reacción de formación de acetal entre benzaldehído y etilenglicol. Una versión de los híbridos en que la superficie había sido pasivada con grupos metilos también fue obtenida. Las propiedades de los materiales híbridos pasivados fueron comparadas, para poder estudiar el efecto de la polaridad de la superficie del soporte sobre la actividad catalítica.

En el Capítulo 4 se describe la síntesis de órgano-catalizadores híbridos obtenidos por anclaje de precursores de silicio funcionalizados con grupos básicos sobre un soporte del tipo MCM-41. Los catalizadores han sido caracterizados y empleados en diferentes reacciones de formación de enlaces C-C, como la condensación de Knoevenagel y la adición de Michael. Finalmente, los catalizadores híbridos han sido empleados en la condensación entre furfural y metil isobutil cetona. El catalizador más activo ha sido seleccionado para ser funcionalizado posteriormente con nanoparticulas de paladio y empleado en un proceso catalítico en cascada. Mecanismos de reacción han sido propuesto para cada 
proceso catalítico. El efecto beneficioso debido a la presencia de los grupos silanoles en la superficie del soporte también fue analizado.

En el Capítulo 5, la síntesis de catalizadores híbridos multi-funcionales fue descrita. Basándose en los resultados obtenidos en el Capítulo 4, se ha preparado un catalizador que presente grupos aminopropil- y nanopartículas de paladio. Las propiedades estructurales y texturales han sido estudiadas. Además, a través de la microscopia electrónica de transmisión, la distribución dimensional de las nanoparticulas ha sido estimada, resultando en un tamaño medio equivalente a la dimensión de los canales mesoporosos del soporte, MCM-41. El material ha sido empleado como catalizador multi-funcional en un proceso tándem basados en la condensación entre furfural y metil isobutil cetona y la hidrogenación consecutiva del producto formado. La influencia de distintas variables sobre la actividad catalítica ha sido estudiada. Los resultados de estas pruebas preliminares permitirán planear en el futuro un estudio más amplio sobre el potencial de este catalizador en un reactor de lecho fijo, en continuo. Además, se han descrito resultados preliminares sobre la síntesis de catalizadores multi-functionales que presentan centros catalíticos básicos y complejos organometalicos soportados. 


\section{Resum}

El treball de recerca descrit en aquesta tesi doctoral es va desenvolupar en el marc del projecte europeu MULTI2HYCAT (grant agreement N. 720783) i se centra en la síntesi i la caracterització de catalitzadors híbrids mono i multifuncionals amb àcid, base o redox actius llocs. S'han desenvolupat diverses línies d'investigació en paral·lel per dissenyar múltiples catalitzadors híbrids per a diferents processos catalítics, basant-se en les necessitats dels socis industrials.

A causa del caràcter col-laboratiu del projecte, cada soci acadèmic es va centrar principalment en un aspecte de tot el procés. Institut de Tecnologia Química (ITQ-CSIC), on es va desenvolupar aquesta tesi, està principalment centrat en el disseny i síntesi de catalitzadors híbrids. Per això, part dels resultats de caracterització reportats al Capítol 3 s'han dut a terme a la Università del Piemonte Orientale (IT), durant una estada d'un mes. Alguns dels resultats catalítics reportats al Capítol $3 \mathrm{i}$ al Capítol 5 han estat realitzats per la Universitat de Southampton (Regne Unit).

En el Capítol 3, s'ha descrit la síntesi de dos catalitzadors heterogenis híbrids que presenten molècules d'àcid aril-sulfònic en la composició. En un d'ells, l'anell aromàtic presentarà àtoms de fluor en posició $2,3,5,6$. S'han dut a terme dues estratègies de síntesi multi-etapes, a través de la síntesi dels precursors alkoxi-silans, mitjançant processos de condensació al costat d'un precursor de sílice (en absència d'agents directors d'estructura, a pH neutre i temperatures baixes) i d'una reacció de tethering. Els materials híbrids han estat caracteritzats mitjançant diferents tècniques. Les propietats texturals, l'estabilitat tèrmica i la composició química dels catalitzadors ha sigut estudiada. A més, molècules sondes han estat adsorbides en els materials híbrids i les interaccions entre ells han estat estudiades mitjançant espectroscòpies FTIR i RMN multi-nuclear. El catalitzador híbrid en que l'anell aromàtic estava fluorat va resultar ser el més actiu catalíticament en la reacció de formació d'acetal entre benzaldehid i etilenglicol. Una versió dels híbrids en que la superfície havia estat pasivada amb grups metilos també va ser obtinguda. Les propietats dels materials híbrids passivats van ser comparades, per poder estudiar l'efecte de la polaritat de la superfície del suport sobre l'activitat catalítica.

En el Capítol 4 es descriu la síntesi d'organo-catalitzadors híbrids obtinguts per ancoratge de precursors de silici funcionalitzats amb grups bàsics sobre un suport del tipus MCM-41. Els catalitzadors han estat caracteritzats i empleats en diferents reaccions de formació d'enllaços C-C, com la condensació de Knoevenagel i l'addició de Michael. Finalment, els catalitzadors híbrids han estat emprats en la condensació entre furfural i metil isobutil cetona. El catalitzador més actiu ha estat seleccionat per a ser funcionalitzat posteriorment amb nanoparticules de pal- ladi i emprat en un procés catalític en cascada. Mecanismes de reacció han estat proposat per a cada procés catalític. L'efecte beneficiós a causa de la presència dels grups silanols en la superfície de suport també va ser analitzat. 
En el Capítol 5, la síntesi de catalitzadors híbrids multi-funcionals va ser descrita. Basant-se en els resultats obtinguts en el Capítol 4, s'ha preparat un catalitzador que presenti grups aminopropil- i nanopartícules de pal-ladi. Les propietats estructurals i texturals han estat estudiades. A més, a través de la microscòpia electrònica de transmissió, la distribució dimensional de les nanoparticulas ha estat estimada, resultant en una grandària mitjana equivalent a la dimensió dels canals mesoporosos del suport, MCM41. El material ha estat emprat com a catalitzador multi-funcional en un procés tàndem basats en la condensació entre furfural i metil isobutil cetona i per la hidrogenació consecutiva del producte format. La influència de diferents variables sobre l'activitat catalítica ha sigut estudiada. Els resultats d'aquestes proves preliminars permetran planejar en el futur un estudi més ampli sobre el potencial d'aquest catalitzador en un reactor de llit fix, en continu. A més, s'han descrit resultats preliminars sobre la síntesi de catalitzadors multi-functionals que presentan centres catalítics bàsics i complexos organometàl-lics suportats. 


\section{Acknowledgements}

I come to the end of my $\mathrm{PhD}$ feeling like a Scientist. I've learnt many things and had the chance to work in very stimulating environments, discussing with top-shelf co-workers. I've lived in different countries, gained enriched points of view of several things, learnt a new language and, most importantly, improved as a person. Before turning page to the next chapter of my life, I want to thank the people that have contributed to this success.

First and foremost, I would like to thank Dr. Urbano Diaz for his mentorship. Along these years, he encouraged me to pursue almost every idea I had, always providing great advices whenever needed and pointing out weak points of my reasoning. I deeply thank him for the interest he put into my formation, allowing my attendance to conference and providing me with the opportunity of actively participate in project meetings, which has been highly important for developing my ability to work in a consortium. On a personal level, I thank Urbano for the enthusiasm he showed for our joint research, which gave me the confidence I needed to work at my best. I believe positive feedbacks are essential, in the long run, and I've been lucky to have those. Lastly, I thank Urbano for his support, especially in the last year of my $\mathrm{PhD}$ thesis. His flexibility, encouragement and in general his friendship, helped me pushing through tough times and getting to the end of my Doctorate.

I would like to thank Prof. Fernando Rey for the opportunity he gave me to enroll as $\mathrm{PhD}$ student at ITQ and for making me feel at home there, since the beginning. I always found discussing scientific results with him very inspiring and insightful as well as just fun, whether this happened in his office or randomly in the corridor. I want to thank him for making my stay at MIT possible. It has been one of the significant times of my life and, for this, I truly owe him.

I want to thank all the people in ITQ. Moving to a foreign country can be challenging, but since my very first day at the institute, I found friendly people, ready to help me with anything, from setting up a reaction to discuss chemistry-related doubts to helping me dealing with personal matters in Spanish, whenever my language skills lacked. In addition to the exceptional scientific level of ITQ, from which I greatly benefited and that will be crucial to my future career, I will remember the time in Valencia as one of the best of my life. I want to thank Candela and Chelo, for the time spent working together and for their help, which came every time I needed it. I thank Vicent, Amparo, Andrès and Nuria. The working environment that we developed in Sintesis 1 was truly special and I have to say that I feel really lucky to have ended up in this lab. I want to thank Pili, who is a true friend in the broadest sense of the word. I want to thank Sebas, Edi, Chema, Alejandro, Aidè, Carla, Marcos, Eva, Elena, Ivan, Irene, Josè, for your friendship and for all the good times spent together during coffee-breaks and lunches, at conferences and, not less important, saliendo de fiesta. I want to thank all other people in ITQ from technicians to people I just had the occasional chat in the corridors. Small things such as this did contribute a great deal into how I felt at ITQ. 
In different measure I owe a thank you to all people mentioned above, for teaching me Spanish patiently, one almuerzo (or one cerveza) at the time!

I would like to thank Prof. Enrica Gianotti, for being a mentor and a friend. I applied for a $\mathrm{PhD}$ position at ITQ on her advice, and it has been one of the best choices I've made in my life. Moreover, during my $\mathrm{PhD}$ years, the scientific collaboration with her greatly contributed to the results I've been able to achieve. I thank her hosting me at Università del Piemonte Orientale in 2018. Most importantly, I thank her for the personal guidance and for the straight-to-the-point advices she is always keen to provide, which mean a great deal to me.

I would like to thank Prof. Yuriy Roman-Leshkov for hosting me at the Chemical Engineering Laboratory at the Massachusetts Institute of Technology. In the six months I spent there I learned a lot, both from the scientific and personal points of view.

I would like to thank all members of the MULTI2HYCAT consortium, for the stimulating environment they created, which really taught me a lot. A special thanks goes to Giovanni for clarifying my doubts on organic chemistry, which have been quite a few, along the years.

I would like to thank la Caixa foundation for my $\mathrm{PhD}$ scholarship.

I would like to thank my family, for supporting and encouraging every choice I make and for accepting me as I am, always. The most important things I learnt in my life, I learnt thanks to you!

Finally, I would like to thank Corinna, whose presence is always the half-full part of my glass. 
A Mattia. 



\section{Table of Contents}

1. Introduction to Functional Hybrid Catalysts 1

1.1. Hybrid organic-inorganic catalysts 5

1.2. Metal-Organic Frameworks (MOFs)

1.3. Mesoporous silica-based hybrid materials $\quad 10$

1.3.1. Post-synthetic modification (Grafting) 11

1.3.2. Co-condensation method ("one-pot") 12

1.3.3. Periodic Mesoporous Organosilicas (PMOs) 14

1.4. Sol-gel chemistry and hybrid materials 17

1.4.1. Hydrolysis and condensation of silicon alkoxides 18

1.4.2. Fluoride catalyzed hydrolysis and condensation 20

1.4.3. Surfactant mediated sol-gel synthesis 22

1.4.4. Handling silyl-derivatives 23

1.5. Silica-based hybrids in heterogeneous catalysis 25

1.5.1. Acid catalysts 25

$\begin{array}{lll}\text { 1.5.2. Base catalyst } & 27\end{array}$

1.5.3. Organometallic complexes 30

1.6. Silica-based multi-functional hybrid catalysts 35

1.7. References 41

2. General Objectives $\quad 49$

3. Organosiliceous Hybrid Materials Functionalized with Aryl-Sulfonic Acid Groups $\quad 55$

3.1. Sulfonic acid groups in heterogeneous catalysis 57

3.1.1. Sulfonic acids supported onto mesoporous carbons 58

3.1.2. Sulfonated mesoporous polymers 60

3.1.3. Sulfonic acids supported onto silica-based materials 61

3.2. Scope of this work 68

3.3. Experimental 70

$\begin{array}{lll}\text { 3.3.1. } & \text { Synthesis of the materials } & 70\end{array}$

$\begin{array}{lll}\text { 3.3.2. Catalytic test } & 71\end{array}$

$\begin{array}{ll}\text { 3.3.3. Characterization } & 72\end{array}$ 
3.4. Results and discussion $\quad 74$

3.4.1. Synthesis of the hybrid catalysts $\quad 74$

3.4.2. Characterization 76

3.4.3. Adsorption of probe molecules followed by spectroscopies (FTIR and NMR)

3.4.4. Catalytic activity 89

3.4.5. Hydrophobic $\mathrm{HSO}_{3}-\mathrm{CH}_{3} \mathrm{NOS}-\mathrm{F}$ and $\mathrm{HSO}_{3}-\mathrm{CH}_{3} \mathrm{NOS}-\mathrm{F}$ hybrid materials 91

3.5. Conclusions 98

$\begin{array}{ll}\text { 3.6. References } & 101\end{array}$

\section{Hybrid Mesoporous Silica-Supported Base Organocatalysts for C-C Bond}

Formation

4.1. Amine-silanol cooperative catalysis for C-C bond formation 111

4.2. Scope of this work $\quad 117$

4.3. Experimental 119

4.3.1. Synthesis of the materials 119

4.3.2. Characterization 120

4.3.3. Catalytic tests 121

4.4. Results and discussion 123

4.4.1. Synthesis and characterization of the hybrid base catalysts 123

4.4.2. Catalytic activity 132

4.5. Synthesis of silica-supported phosphazene materials (P1) 146

4.6. Conclusions 150

4.7. References 153

5. Synthesis of Multi-Functional Hybrid Catalysts for Cascade Reactions 157

5.1. Silica-supported palladium nanoparticles 161

5.2. Silica-supported Pd organometallic complexes 165

5.2.1. Pd-based N-Heterocyclic Carbenes (NHCs) 165

5.2.2. Pd-BINAP complexes 167

5.3. Furfural as "green" building block 169

5.4. Scope of this work 171

5.5. Experimental 173

5.5.1. Synthesis of the materials 173

$\begin{array}{ll}\text { 5.5.2. Characterization } & 174\end{array}$

xvi 
5.5.3. Catalysis 174

5.6. Results and discussion 175

5.6.1. Bi-functional catalysts featuring Pd nanoparticles $\quad 175$

5.6.2. Bi-functional catalysts featuring palladium complexes 182

5.7. Conclusions 189

5.8. References 191

6. General Conclusions $\quad 195$

Appendix 1: Characterization Techniques $\quad 203$

Appendix 2: List of Figures and Tables $\quad 213$

$\begin{array}{ll}\text { Scientific Contribution } & 223\end{array}$ 



\section{Chapter 1}

\section{Introduction to Functional Hybrid Catalysts}

The synthesis of hybrid organic-inorganic materials provides powerful tools to incorporate catalytically active functional groups within inorganic frameworks. Silica-based hybrids are especially suitable for this task, due to their mechanical stability, tunable textural properties and reactive surface. In this Chapter, a brief introduction to silica-based hybrid catalysts is reported. Examples of mono-functional catalysts, baring acid, base, organometallic functionalities are reported. Examples regarding multi-functional catalysts are also described with particular focus on the synthetic strategies that different authors developed to incorporate multiple functional groups, often incompatible with each other, through multi-step synthetic pathways. The chemistry of sol-gel synthesis of silica is also reported. Hydrolysis/condensation mechanisms are described, as well as acid, base and fluoride catalyzed sol-gel. 

Catalysis plays a crucial role in the chemical industry since many decades. Approximately, $85-90 \%$ of the products of chemical industry are made in catalytic processes. They are indispensable in the production of transportation fuels, with about 440 oil refineries heavily relying on them (data of the year 2007) and in the production of bulk and fine chemicals in all branches of the chemical industry. They are key players in the prevention of pollution, in the abatement of automotive or industrial exhaust and they provide environmental and economic advantages by enhancing the selectivity toward the desired product, in turn avoiding or limiting the production of unwanted byproducts. Catalysts offer alternative, energetically favorable mechanisms to non-catalyzed reaction, therefore enabling processes to be carried out under industrially feasible conditions of temperature and pressure. Catalysts come in many forms, from atoms and small molecules to macromolecules such as enzymes and crystalline structures such as zeolites and metal oxides. Moreover, they can be employed in different conditions: liquid, gaseous or at the surface of solids. ${ }^{1}$

In heterogeneous catalysis, solid materials catalyze reactions of molecules in gas or liquid phase. They provide several advantages over homogeneous catalysts, such as the ease of separation from the reaction mixture and consequent better recyclability and reuse. In addition, solid frameworks and macromolecules bring about additional properties, compared to homogeneous catalysts, which have been exploited by scientists for several decades. Examples involve the shape selectivity of microporous zeolites that, due to the shape of the cavity in which the active site is located, allows for the catalytic activation of a specific reactant or promote the formation of a specific product by stabilizing the transition state that precedes the desired product. Nature pioneered this strategy with much greater success, specifically with enzymes, in which the way they are folded onto themselves allows for exceptional specificity toward certain reactants and selectivity toward certain products. ${ }^{2-5}$ (Fig. 1.1) Another instance is the synthesis of supported noble metal nanoparticles. Due to their small size, nanoparticles expose a relatively large surface area that, when they are used in homogeneous phase, cause them to aggregate and sinter, in turn lowering the surface area exposed and lowering their free energy. By depositing the nanoparticles onto solid supports (often porous), chemists have been able to limit sintering, allowing the use of these catalysts over multiple cycles and easing their separation from the reaction mixture. ${ }^{6-8}$ Even further, in the last decades scientists have been able to coordinate single atoms of various metals within a solid support, with major advantages in terms of catalytic activity and atom economy. ${ }^{9,10}$

Among the different types of heterogeneous catalysis, hybrid organic-inorganic catalysts provide some unique properties, compared to purely inorganic catalysts (e.g. zeotypes) and purely organic ones (e.g. resins). In fact, they combine the advantages of inorganic solids (high mechanical, thermal, and structural stability) and organic molecules or macromolecules (flexibility and functionality). 
A

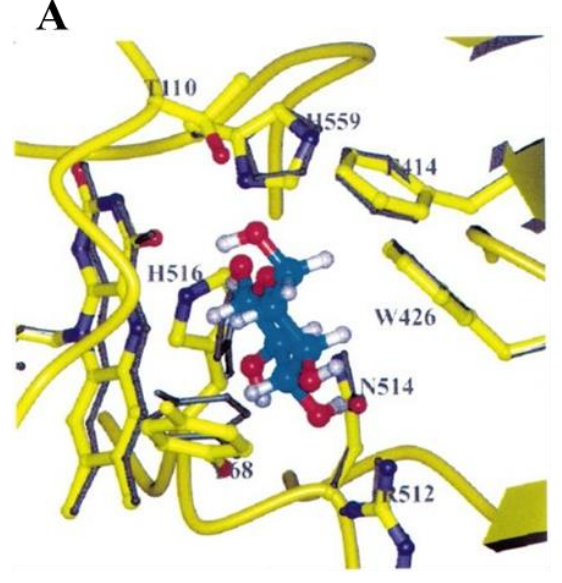

C
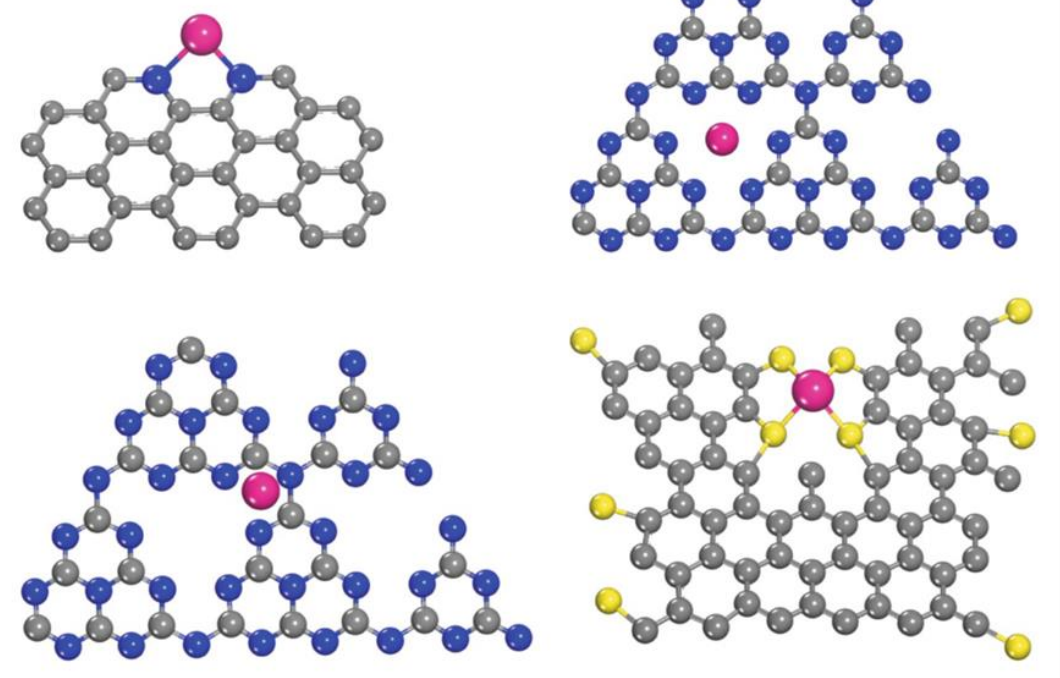

Figure 1.1: Stereoview of the active site of glucose oxidase with the modelled substrate $\beta$-dglucose (adapted from ref. 4). B: The structure of $\gamma$-valerolactone within zeolite pores as obtained using in situ X-ray diffraction data (adapted from ref. 5). C: Single Pt atoms bounded to $\mathrm{N}$ - rich or $\mathrm{S}$ - rich regions in doped carbonaceous supports (adapted from ref. 10). 


\subsection{Hybrid organic-inorganic catalysts}

In general, the concept of hybrid material refers to a solid composed of two types of building blocks, an inorganic one and an organic one, mixed with each other at the nanometric scale. By cleverly combining different organic and inorganic precursors, many authors have been able to guide their self-assembly, achieving hybrids featuring unique properties for applications as advanced materials in several fields. In the last few decades, these types of materials underwent tremendous development, thanks to soft solgel and self-assembling micellar processes (Fig. 1.2). ${ }^{11}$ Hybrid materials can be classified according to the interactions between organic and inorganic building blocks. Quite logically, the strength and the directionality of chemical bonds holding them together are crucial aspects to consider in the pursuit of designing a hybrid with specific properties.

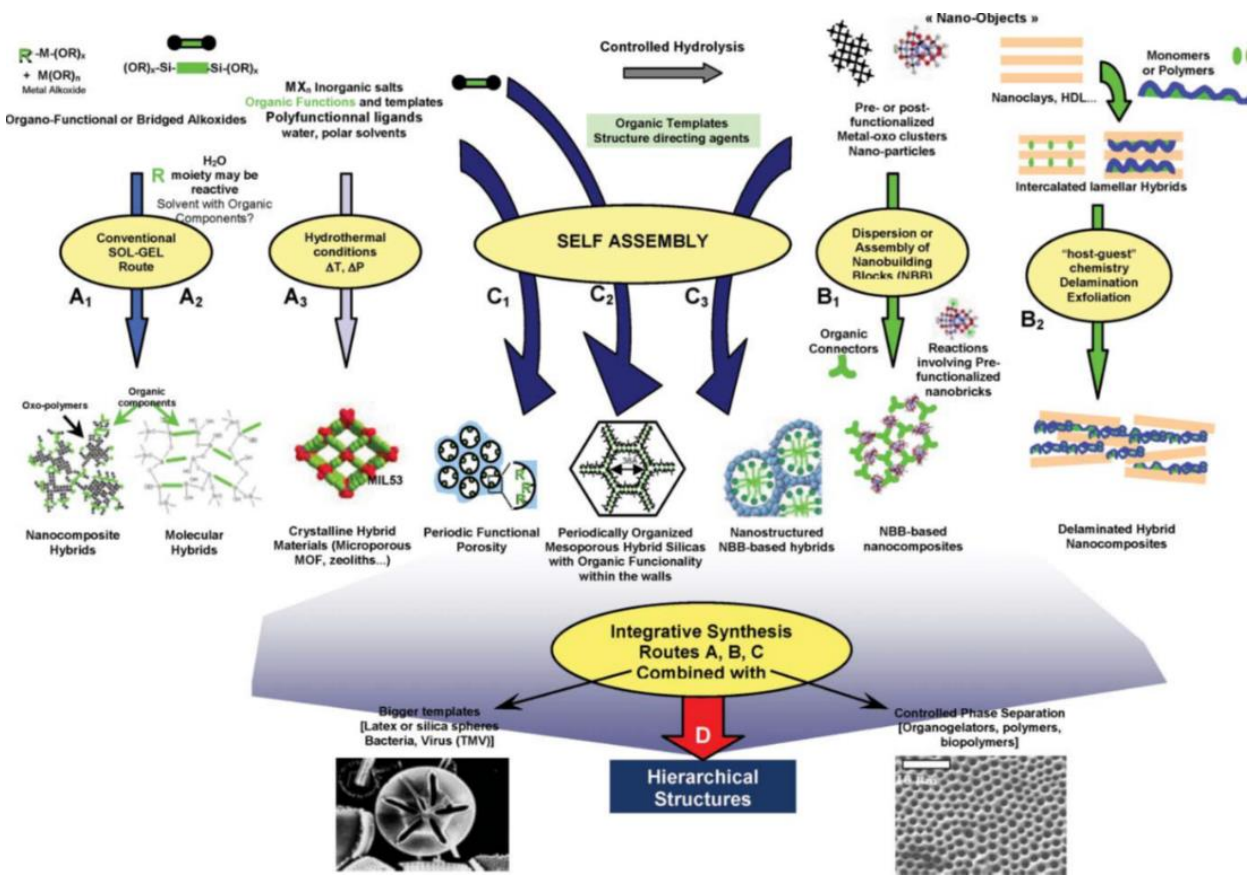

Figure 1.2: Scheme of the main chemical routes for the synthesis of organic-inorganic hybrids. Adapted from ref. 11. 
Sanchez and Ribot classified as Class I hybrids those in which weak bonds (hydrogen or van der Waals bonding) give cohesion to the whole structure (Fig. 1.3). Organic dies have been imbedded in amorphous sol-gel matrixes, in order to improve their stability and avoid the formation of dimers or oligomers. ${ }^{12,13}$ Inorganic fillers have long been imbedded into organic polymeric matrixes in order to improve their mechanical properties. However, traditional filler blending occurs under high viscosity conditions, which lead to partial agglomeration of the inorganic particles, lowering the matrix-filler interactions. By generating the inorganic particles in-situ by sol-gel chemistry, a much better dispersion can be achieved. ${ }^{14,15}$ Wet or dry impregnation have been implemented to obtain Class I hybrids, as well. ${ }^{16}$
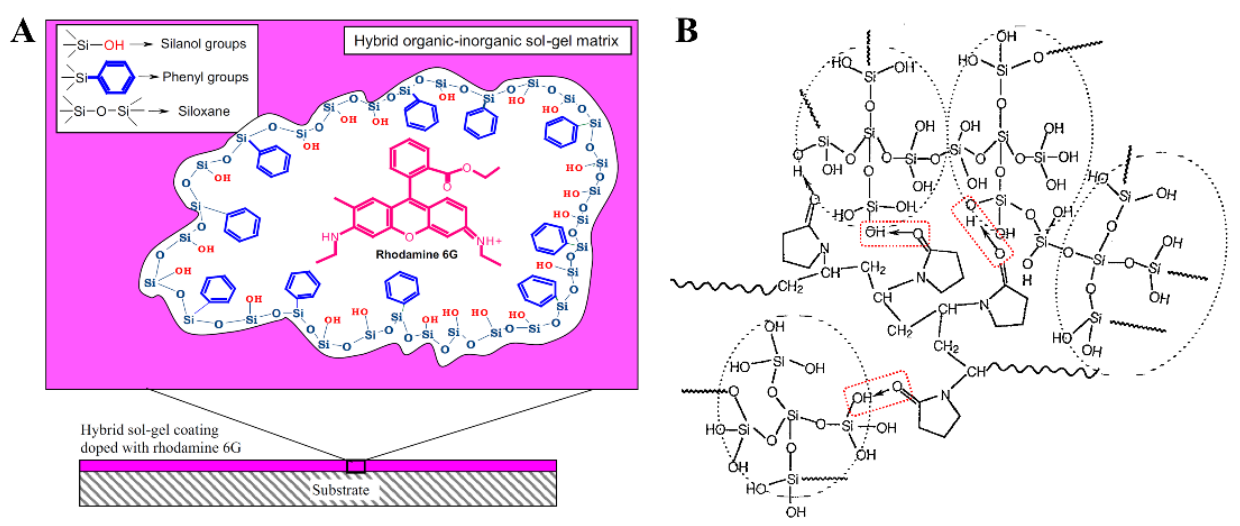

Figure 1.3 A: Schematic representation of the porous structure of a phenyl modified silica matrix doped with Rhodamine 6G molecules (adapted from ref. 13). B: Structure of PVP-silica hybrid material (adapted from ref. 14).

In Class II materials, the two phases are linked together through strong chemical bonds (covalent, coordination or ionic bonding). ${ }^{11,16,17}$ This type of hybrids are of special interest for catalytic applications. In fact, while weak bonds between organic and inorganic building blocks would be susceptible to breakage in the reaction media, with consequent loss of structural properties and catalytic activity, strong bonds would favor the preservation of the hybrid catalysts, allowing for using the catalyst over multiple cycles. There are two main families of hybrid materials belonging to class II that are implemented as heterogeneous catalysts: metal-organic frameworks and mesoporous silica-based hybrids. Other types of hybrid materials have been described, e.g. organozeolites ${ }^{18}$ silicagraphene composites ${ }^{19}$ and laminar metal oxides featuring organocatalysts in the interlaminar space. ${ }^{20}$ However, they are less commonly used in heterogeneous catalysis and less related to the research described in this Thesis. For that, after a brief introduction of MOFs, the discussion will focus on silica-based hybrids. 


\subsection{Metal-Organic Frameworks (MOFs)}

Within the realm of reticular chemistry, metal-organic frameworks (MOFs) are the most prominent class of materials. ${ }^{21}$ Yaghi et al. classified as MOFs those coordination polymers featuring crystalline structure, high surface area, specific pore size distribution and high stability, consequent to the strong metal-ligand interactions. MOFs are composed by inorganic poly-nuclear clusters (Secondary Building Units or SBU) and organic linkers bounded together by strong coordination bonds. ${ }^{22}$ The term SBU was originally used in the description of zeolites, where primary building units $\left(\mathrm{TO}_{4}\right.$ tetrahedra) form larger periodically recurring structural arrangements. ${ }^{23}$ Inorganic clusters act as framework nodes, bounding organic linkers and allowing for the development on mono- bi- and tridimensional structures. Appropriate choices of metals and ligands, together with specific synthetic conditions can promote the formation of one structure or another. Different metals have been successfully implemented as building block in the synthesis of MOFs, both as single atoms or clusters: alkaline, earth alkaline, transition block metals as well as rare earth metals. Under specific conditions, each metallic unit forms a specific SBU featuring coordination vacancies, available to coordination by the organic ligands. Number and spatial position of the coordination vacancies vary between different SBUs, which, in turn, leads to the formation of differently structured materials. ${ }^{24}$ Among the many possible SBUs, two of the most widely used to synthetize MOFs are those present in MOF-5 $5^{25}$ and MIL-88 ${ }^{26}$ (Materials Institute Lavoisier), reported in Fig 1.4.
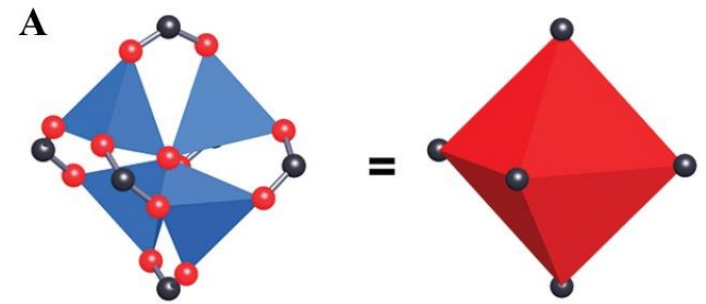

B

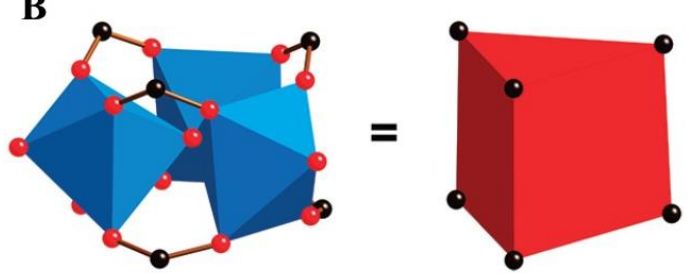

Figure 1.4 A: SBU of MOF-5 $\left(\mathrm{Zn}_{4} \mathrm{O}\right)$. B: SBU of MIL-88 $\left(\mathrm{Cr}_{3} \mathrm{O}\right) .^{27}$ 
Depending on the inorganic precursor, the organic ligand and the synthesis conditions, more complex SBUs can be obtained. Concerning the organic ligands, in recent years, most studies have been focused on organic molecules baring carboxylic acid groups, which can strongly coordinate metal nodes through the oxygen atoms of the carboxylate anion (Fig. 1.5).$^{28,29}$ Relevant examples include rigid aromatic moieties such as terephthalic acid ${ }^{30}$, but also more flexible aliphatic molecules such as succinic acid ${ }^{31}$ and glutaric acid ${ }^{32}$. Others functional groups have been exploited as ligands to coordinate metal nodes for the synthesis of MOFs. Examples include phosphonic acids, pyridine- and imidazole-based moieties. ${ }^{22}$<smiles>O=C(O)c1ccc(C(=O)O)cc1</smiles><smiles>O=C(O)c1cc(C(=O)O)cc(C(=O)O)c1</smiles><smiles>O=C(O)c1cc(C(=O)O)cc(C(=O)O)c1</smiles>

$\mathrm{H}_{3} \mathrm{BTC}$<smiles>Nc1cc(C(=O)O)ccc1C(=O)O</smiles><smiles>O=C(O)c1ccc2cc(C(=O)O)ccc2c1</smiles><smiles>c1cc(-c2ccncc2)ccn1</smiles>

BIPY

$\mathrm{NH}_{2}-\mathrm{H}_{2} \mathrm{BDC}$

2,6- $\mathrm{H}_{2} \mathrm{NDC}$<smiles>O=C(O)c1cc(O)c(C(=O)O)cc1O</smiles><smiles>O=C(O)c1ccc(-c2cc(-c3ccc(C(=O)O)cc3)cc(-c3ccc(C(=O)O)cc3)c2)cc1</smiles>

Figure 1.5: Various carboxylic acid linkers used for MOF synthesis and a common nitrogen linker group BIPY. ${ }^{29}$

After an initial period, in which most publication on MOFs focused on synthesis methods and discovery of new structures, in the last decade more and more studies are being reported on their possible applications. Relevant examples range from gas storage ${ }^{33}$ and heterogeneous catalysis ${ }^{34}$ to applications exploiting luminescent ${ }^{35}$ or magnetic ${ }^{36}$ properties and controlled drug delivery ${ }^{37}$.

MOFs can display excellent textural properties, with specific surface area values reaching up to $7000 \mathrm{~m}^{2} / \mathrm{g}$, in some cases. ${ }^{38}$ That, together with their tunable surface properties, 
make them ideal candidates for adsorption applications. Reports in the scientific literature include selective adsorption of $\mathrm{CO}_{2},{ }^{39} \mathrm{H}_{2},{ }^{40} \mathrm{CH}_{4}{ }^{41}$ as well as selective adsorption of $\mathrm{H}_{2}$ and $\mathrm{O}_{2}$ fed into the reactor mixed with $\mathrm{N}_{2}$ and $\mathrm{CO}$, and separation ${ }^{42}$ of propylene from a propylene/propane mixture ${ }^{43}$. All of these separation processes involve very high quantity of gases and high amount of energy required. Therefore, possible future applications of more effective adsorbent on an industrial scale will have a big impact in the progressive shift to a more environmentally friendly chemical industry.

High surface area, tunable pore size and relatively high chemical and thermal stability make MOFs exploitable as heterogeneous catalysts. Many articles are reported in the scientific literature on reactions such as oxidations, epoxidations, aldol condensations, hydrogenations among others. ${ }^{44} \mathrm{MOFs}$ ' catalysis can be classified based on which part of the hybrid catalyst constitute the active site: intrinsic reactivity of structural metallic sub-domains, coordination vacancies of the metal nodes ${ }^{45}$, metal nanoparticles located in the framework cavities, ${ }^{46}$ and reactive functional groups located on the organic ligands, ${ }^{47}$ mainly (Fig. 1.6).

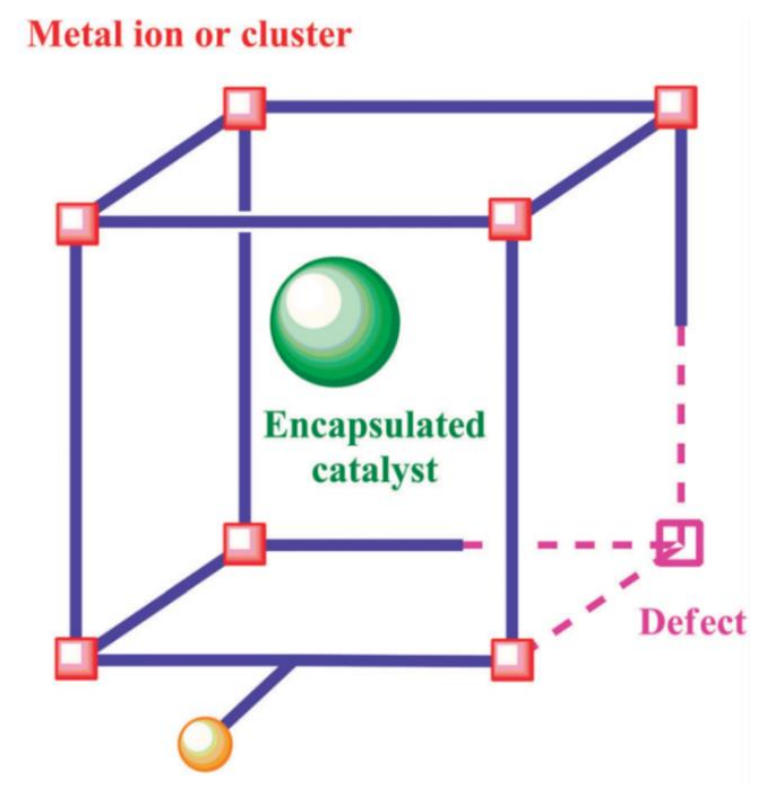

Functionalized linker

Figure 1.6: Different active sites available in MOFs responsible for catalysis. ${ }^{48}$ 


\subsection{Mesoporous silica-based hybrid materials}

Since the development of M41S materials by the Mobil Oil Company in 1992, porous materials with large specific surface areas has been a field of extensive research, particularly with regard to potential applications in areas such as catalysis, adsorption, gas storage, chromatography, controlled drug-delivery and sensor technology. Like microporous crystalline zeolites, this class of materials feature very large specific surface areas, ordered pore systems and well-defined pore size distributions. However, unlike zeolites, M41S materials exhibit amorphous pore walls and their pore diameters is comprised between 2 and $10 \mathrm{~nm}$. The most representative materials belonging to this class include silica solids MCM-41 (with a hexagonal arrangement of the mesopores), MCM48 (with a cubic arrangement of the mesopores), and MCM-50 (with a laminar structure). ${ }^{49}$ (Fig. 1.7)

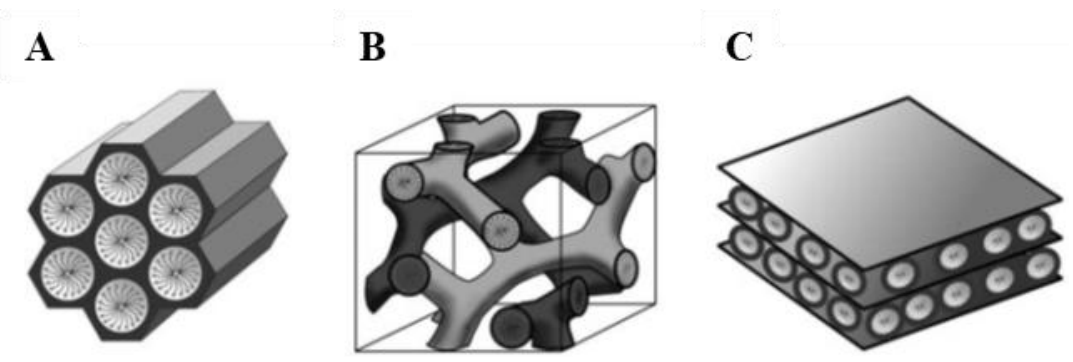

Figure 1.7: Structures of mesoporous M41S materials: MCM-41 (A), MCM-48 (B) and MCM$\mathbf{5 0}(\mathbf{C}){ }^{49}$

Not long after their discovery, researchers began attempting to incorporate organic components within an inorganic silica framework to achieve symbiosis of the properties of both components. The combination of the properties of organic and inorganic building blocks within a single material is highly attractive. In fact, this allows for the possibility of combining the enormous variety of functional groups, typical of organic chemistry, with the advantages of a thermally stable and robust inorganic substrate. This is particularly appealing for applications in heterogeneous catalysis. The symbiosis of organic and inorganic components can lead to materials with considerably different properties from those of their individual, isolated components. Silica-based materials also allow for adjustment of the polarity of the pore surface either by the addition of organic building blocks in the synthesis medium or by post-synthetic reaction of the surface silanol groups with organic siloxanes, that can act as passivating agents. Using the same approaches, it is possible to functionalize the silica matrix with specific organic functionalities such as $\mathrm{C}=\mathrm{C}$ multiple bonds, alcohols, thiols, sulfonic and carboxylic acids, amines. This aspect is central in the frame of this Thesis. Sol-gel chemistry provides a versatile and effective 
way of anchoring organic moieties to a porous solid silica support. As it will be shown below, these organic moieties (e.g. acidic, basic, organometallic complexes) can be catalytically active for a specific reaction and being selective toward the formation of one product, instead of another. Bounding them to a solid support, opens up to the possibility of carrying out reactions under heterogeneous regime, allowing for recovery and reuse of these valuable catalyst over multiple cycles, easing product separation and purification. ${ }^{1,49}$ Silica-based hybrids can be achieved by (i) post-synthetic modification of the pore surface of a purely inorganic silica material (grafting), (ii) co-condensation of a silica precursor and a silyl-derivative baring the functional group of interest ("one-pot" approach), (iii) condensation of bi-silylated precursors featuring the functional group of interest in the linker between the two alkoxy-silyl groups (alone or together with a minimal amount of tetra-alkoxysilyl precursor), leading to a material in which the organic functionalities are imbedded within the pore walls.

\subsubsection{Post-synthetic modification (Grafting)}

Grafting refers to the post-synthesis modification of the surfaces of mesostructured silica phases with organic groups. This process is mainly carried out by reaction of organosilanes of the type $\mathbf{R S i}\left(\mathrm{OR}^{\prime}\right)_{3}$ with the free silanol groups of the pore surfaces. Less frequently chlorosilanes $\mathrm{ClSiR}_{3}$ or silazanes $\mathrm{HN}\left(\mathrm{SiR}_{3}\right)_{2}$ are used. (Fig. 1.8)

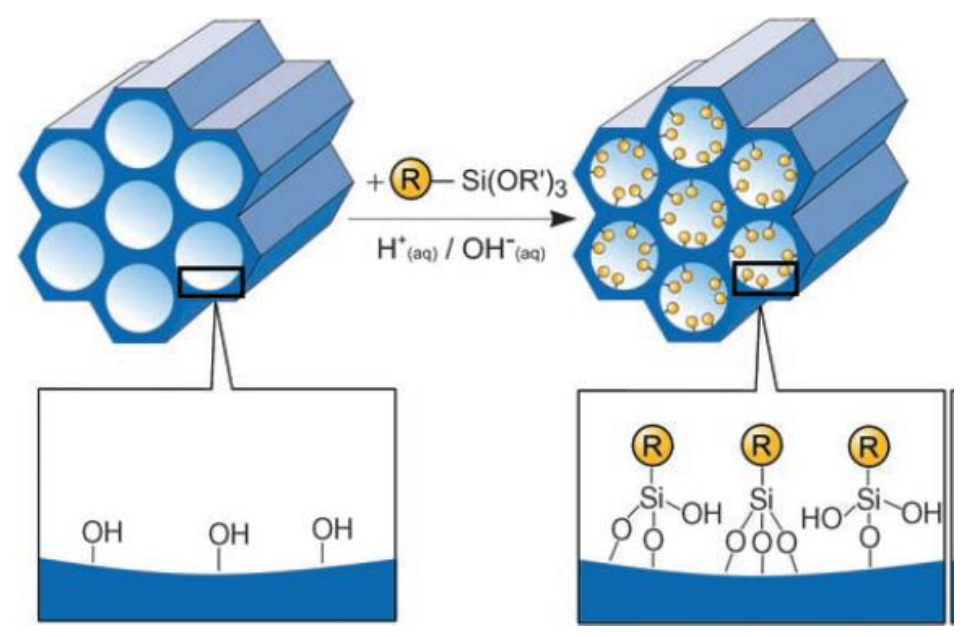

Figure 1.8: Grafting of a generic silyl-derivative $\operatorname{RSi}\left(\mathrm{OR}^{\prime}\right)_{3}$ onto a mesoporous silica pore walls. $^{49}$ 
In principle, by varying of the organic residue $\mathrm{R}$, functionalization with different organic groups can be achieved. By implementing this approach, the mesostructure of the starting silica phase is usually retained. On the other hand, a reduction in the porosity of the hybrid, compared to the parent mesoporous silica, is usually observed, proportionally to the size of the grafted organic moieties. Furthermore, the organic silyl-derivatives might preferentially react with the surface silanol groups that they will encounter first, i.e. those located at the pore mouth or in the initial part of the mesoporous channels. This effect will be modulated by several factor. Firstly, the reactivity of the alkoxy groups bounded to the silicon atom will determine the ease with which hydrolysis/condensation reactions will occur. The diffusion of the silyl-derivative compounds through the mesopores, primarily determined by its size, relative to the pores diameter, and by the chemical affinity between the organic moieties and surface silanol groups. Lastly, while in an initial stage of the grafting, silyl-derivatives are allowed to diffuse through the pore channels before the catalyst (acid or base) is added into the mixture, molecules baring acidic or base functionalities can catalyze their own hydrolysis and condensation before reaching optimal diffusion, leading to a worse distribution of organic moieties throughout the porous framework. A key advantage, unique to this method, is the fact that the templating agent used for generating the mesopores is entirely removed by calcination prior to the grafting procedure. The grafting approach allows for introducing organic functionalities onto a pre-existing mesoporous silica framework. Consequentially, in these hybrids the organic moieties are expected to be pending from the pore walls, partially occupying the mesoporous channels.

\subsubsection{Co-condensation method (“one-pot”)}

An alternative method to synthesize ordered hybrid organic-inorganic mesoporous silica phases is the co-condensation method. It is possible to prepare mesostructured silica phases by the co-condensation of tetra-alkoxysilanes, typically tetraethyl ortosilicate (TEOS) or tetramethyl ortosilicate (TMOS), together with trialkoxy-organosilanes of the type $\mathbf{R S i}\left(\mathrm{OR}^{\prime}\right)_{3}$ in the presence of structure-directing agents, obtaining materials with organic residues pending covalently from the pore walls (Fig. 1.9).

By using structure-directing agents that are typically implemented in the synthesis of pure mesoporous silica phases (e.g. MCM or SBA silica phases), organo-functionalized silica hybrids can be prepared in such a way that the organic linkers, connecting the functional group of interest to the inorganic support, are pending directly from the pore walls, with the silicon atoms deriving from hydrolysis/condensation of the alkoxy-silyl groups being imbedded into the silica pore walls. Since the organic functionalities are direct components of the silica matrix, pore blocking is not a problem in the co-condensation method, although a slight decrease in the pore diameter can be detected. Moreover, the organic units are generally more homogeneously distributed than in hybrid mesoporous materials obtained by grafting process. 


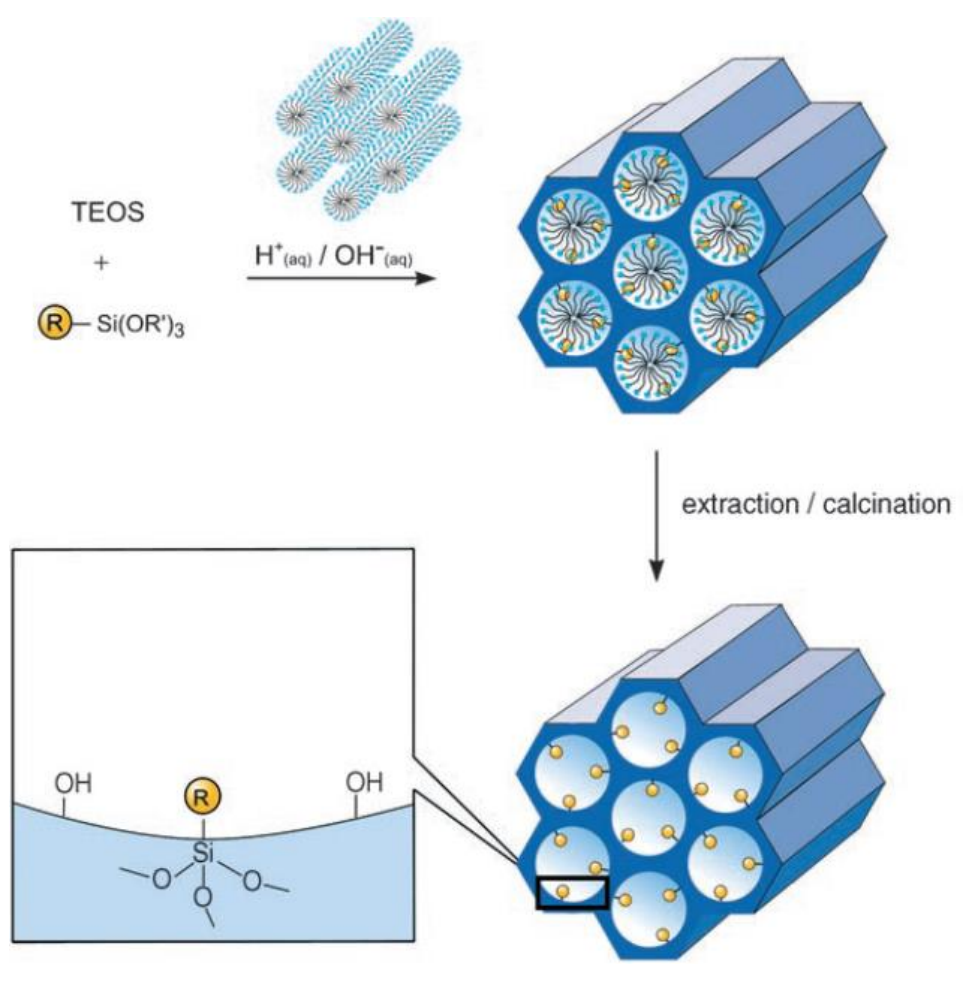

Figure 1.9: Co-condensation between tetraethyl ortosilicate (TEOS) and a generic silyl-derivative compound, $\mathrm{RSi}(\mathrm{OR})_{3}$, followed by calcination/extraction to achieve hybrid organic-inorganic mesoporous silica. ${ }^{49}$

Nevertheless, the co-condensation method presents also a number of disadvantages: the degree of mesoscopic order of the resulting materials tend to decreases with increasing concentration of $\mathrm{RSi}\left(\mathrm{OR}^{\prime}\right)_{3}$ in the synthesis mixture. Consequently, the relative amount of organic moieties in the modified silica phases does not normally exceed $40 \mathrm{~mol} \%$. Furthermore, the yield with which the organic groups can be incorporated into the porewall network is generally not $100 \%$ and it decreases proportionally to the amount of organic silyl-derivative in the synthesis mixture. These observations can be explained by the fact that an increasing proportion of $\mathrm{RSi}\left(\mathrm{OR}^{\prime}\right)_{3}$ in the reaction mixture promotes homocondensation reactions, favoring the formation of oligomers. These phenomena are caused by the different hydrolysis and condensation rates of the different silyl-type precursors. It is a potential problem whenever this synthetic strategy is implemented, as the homogeneous distribution of the organic functionalities in the framework cannot be guaranteed. Moreover, exceeding in the loading of organic groups can lead to a reduction 
in the pore diameter, pore volume, and specific surface areas. From a purely methodological point of view, a higher degree of care needs to be taken in the removal of the surfactant, used to generate the mesopores. Calcination cannot be implemented as it would compromise the integrity of the organic groups Si-R. Consequently, template removal is commonly done by continuous extraction, carried out using a soxhlet apparatus. Although fairly effective, an incomplete removal of the template is usually observed.

An additional tool to incorporate specific organic functionalities within silica-based materials are tethering procedures. For that, firstly, mono-silanes $\mathrm{RSi}\left(\mathrm{OR}^{\prime}\right)_{3}$ are incorporated into a silica support (either by co-condensation or grafting). Afterwards, in a postsynthetic step, an organocatalyst featuring the active site of interest and a specific functional group $\mathrm{R}^{*}$ is reacted with the hybrid material. $\mathrm{R}$ and $\mathrm{R}^{*}$ need to be chosen properly, so that they can react and form a covalent bond that can guarantee the anchoring of the organocatalyst. Example of such R/R* pairs include primary amine and isocyanate, reacting to form urea group; thiol and terminal alkene, reacting in presence of a radical initiator to form a thioether; a halide (Br or I) functioning as good leaving group in a $\mathrm{S}_{\mathrm{N}} 2$ reaction and a strong nucleophile.

\subsubsection{Periodic Mesoporous Organosilicas (PMOs)}

PMOs are a class of silica-based organic-inorganic hybrid materials obtained by hydrolysis and condensation reactions of bridged silsesquioxane precursors of general formula $\left(\mathrm{R}^{\prime} \mathrm{O}\right)_{3} \mathrm{Si}-\mathbf{R}-\mathrm{Si}(\mathrm{OR})_{3}$, used as the only silicon source. ${ }^{50,51}$ In contrast with the two methods previously reported (grafting and direct synthesis), in this case, the organic building blocks bear two alkoxy-silyl groups that can largely improve their incorporation in the three-dimensional network as well as their homogeneous distribution throughout the solid (Fig. 1.10 A). Moreover, a major difference between PMOs and silica-based hybrids obtained by co-condensation and grafting is that in the former, active sites are imbedded into the pore walls, rather than pending from the surface of the support. As in the case of the synthesis of pure silica mesophases, the use of a surfactant in the presence of bi-silylated organosilica precursors allows obtaining materials featuring ordered mesopores, narrow pore size distribution and in which the organic bridges are integral components of the silica network. ${ }^{49}$

Although presenting several advantages over grafting and co-condensation, the synthesis of PMOs also bring about challenging aspects, the most notable being the synthesis of the suitable bi-silylated precursors. In fact, alkoxy-silyl groups are highly reactive species and synthesizing complex molecules baring two of such groups and a specific organic residue $\mathrm{R}$, is often a challenging task. Nevertheless, bridged polysilsesquioxanes featuring a wide range of bridging groups have been prepared. In 1999, three research groups independently reported on mesoporous organosilica hybrids from bi-silylated precursors baring methyl or ethyl groups as organic bridges. ${ }^{50,51,53}$ After that, several PMOs were prepared featuring ethylene, ethenylene, phenylene and thiophene as $\mathrm{R}$ groups. 

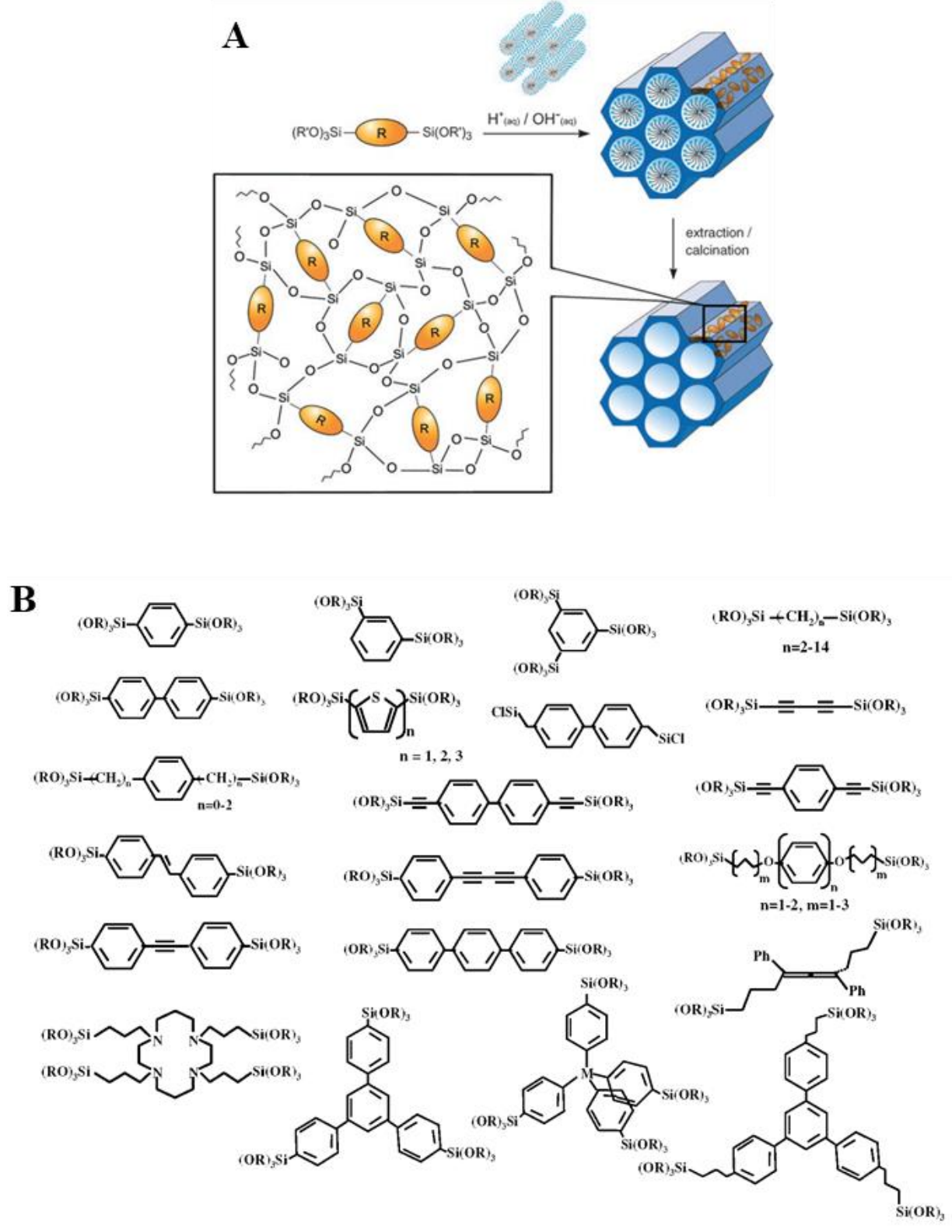

Figure 1.10 A: General synthetic pathway to PMOs. $^{49}$ B: Representative bridged silsesquioxanes. ${ }^{52}$ 
Rigid unsaturated precursors able to form weak interaction, such as $\pi-\pi$, can self-assemble in the synthesis mixture. When hydrolysis/condensation take place, this order can be preserved and layered "crystal-like" structural sub-domains can be included in the final PMO-type materials. The first materials featuring 1,4-phenylene bridges was prepared in 2002. Upon varying the size of the $\mathrm{R}$ residue, materials featuring layers of different width were obtained (5.6 - $11.9 \AA$ А). ${ }^{54}$ Building upon these early studies, a variety of organic groups, ranging from hydrocarbons and heteroaromatics to metal complexes, have been successfully incorporated into PMO hybrids (Fig $1.10 \mathrm{~B}$ ). ${ }^{49,52,55}$ 


\subsection{Sol-gel chemistry and hybrid materials}

In this Section, a description of the sol-gel chemistry of silica is reported, with a specific focus on aspects that are recurrent throughout this Doctoral Thesis and that need to be considered when designing and preparing silica-based hybrid materials. In a general definition, a sol is defined as a colloidal dispersion of particles in a liquid, while a gel consists of a continuous solid skeleton enclosing a continuous liquid phase. The formal definition of sol-gel processing is therefore the growth of colloidal particles and their linking together to form a gel.

Hydrolyzed silica in aqueous solution is constituted by orthosilicic acid, $\mathrm{Si}(\mathrm{OH})_{4}$. Protonation of silanols to form cationic species $\equiv \mathrm{Si}\left(\mathrm{OH}_{2}\right)^{+}$can occur below $\mathrm{pH} 2$, while further hydrolysis involves the deprotonation of a silanol group to form an anionic species $\mathrm{Si}(\mathrm{OH})_{3} \mathrm{O}^{-}$above $\mathrm{pH} 7$, and $\mathrm{Si}(\mathrm{OH})_{2} \mathrm{O}_{2}{ }^{2-}$ above $\mathrm{pH} 12$ (Fig 1.11).<smiles></smiles>

$\mathrm{pH}<2$<smiles>[O-][Si](O)(O)O</smiles>

$\mathrm{pH}>7$<smiles>[O-][Si]([O-])(O)O</smiles>

$\mathrm{pH}>12$

Figure 1.11: Protonation/deprotonation of orthosilicic acid.

Concerning the condensation of silicic units, Iler ${ }^{56}$ divides the polymerization process into three approximate $\mathrm{pH}$ domains: $\mathrm{pH}<2,2-7$, and $>7$. At $\mathrm{pH} 2$, a boundary is present, due the point of zero charge, i.e. surface charge is zero, and the isoelectric point, i.e. electrical mobility of the silica particles, is also zero. A pH of 7, represents another boundary, because both the solubility and dissolution rates are maximized at or above $\mathrm{pH} 7$, and because above $\mathrm{pH} 7$ the silica particles are appreciably ionized. Consequently, particle growth occurs without aggregation or gelation.

Below $\mathrm{pH}$ 2, condensation involves the protonation of a silanol, followed by nucleophilic attack on the silicon atom by a $\equiv \mathrm{SiOH}$ species. Polymerization above the isoelectric point $(\mathrm{pH}>2)$ involves a bi-molecular nucleophilic condensation mechanism $\left(\mathrm{S}_{\mathrm{N}} 2-\mathrm{Si}\right)$, featuring the attack of hydrolyzed, anionic species on neutral species. Due to inductive effects, the most acidic silanols, which are the most likely to be deprotonated, are the most highly condensed species. Consequently, condensation occurs preferentially between more condensed species and less condensed, neutral, species. In other words, monomers and oligomers feed the growth of bigger particles. Specifically, once tetramers are formed, they tend to cyclize due to the proximity of chain ends and further growth occurs by addition of monomer and other low-molecular-weight species to cyclic species to form particles, and by aggregation of particles to form bigger networks. 
Above $\mathrm{pH} 7$, particle growth differs from that below $\mathrm{pH} 7$ by at least two factors. Firstly, while near the isoelectric point no electrostatic repulsion is present, so that growth and aggregation processes occur altogether, at $\mathrm{pH}$ above 7 particles are appreciably charged, which makes particle aggregation unlikely. Secondly, above $\mathrm{pH} 7$ the size dependence of the solubility of silica increases. Consequentially, growth of primary particles continues by Ostwald ripening, a process in which smaller, more soluble particles dissolve and feed the growth on larger, less soluble particles. The growth ceases when the difference in solubility between the largest and smallest particles becomes negligible. ${ }^{57}$

\subsubsection{Hydrolysis and condensation of silicon alkoxides}

Silicate gels are most often synthesized by hydrolyzing the alkoxides dissolved in water and/or their parent alcohols with a mineral acid or base catalyst. ${ }^{58,59}$ Three bimolecular nucleophilic reactions are generally used to describe the sol-gel process:

$$
\begin{aligned}
& \equiv \mathrm{Si}-\mathrm{OR}+\mathrm{H}_{2} \mathrm{O} \longrightarrow \longrightarrow \mathrm{Si}-\mathrm{OH}+\mathrm{ROH} \\
& \equiv \mathrm{Si}-\mathrm{OR}+\equiv \mathrm{Si}-\mathrm{OH} \longrightarrow \mathrm{Si}-\mathrm{O}-\mathrm{Si} \equiv+\mathrm{ROH} \\
& \equiv \mathrm{Si}-\mathrm{OH}+\equiv \mathrm{Si}-\mathrm{OH} \longrightarrow \mathrm{H}_{2} \longrightarrow
\end{aligned}
$$

The first reaction involves the hydrolysis of the alkoxy residues and the release of an alcohol molecule ROH. Reactions (2) and (3) represent nucleophilic substitutions in which the oxygen of a hydroxyl group attacks a silicon atom to form a $\mathrm{Si}-\mathrm{O}-\mathrm{Si}$ species, with release of $\mathrm{ROH}$ or $\mathrm{H}_{2} \mathrm{O}$, respectively. The hydrolysis reaction can be acid or base catalyzed. Acid catalysts protonate the alkoxide group, making a better leaving group $(\mathrm{ROH})$ that does not require a proton transfer in the transition state. Base catalysts contributes to the reaction rate by increasing the concentration of strongly nucleophilic $\mathrm{OH}^{-}$ species.

The condensation reaction is dependent on the acidity of the silicate reactants. At $\mathrm{pH}$ values above 2 , acidic silanols are deprotonated and strong nucleophiles, $\equiv \mathrm{SiO}^{-}$, are created. Below about $\mathrm{pH} 2$, weakly acidic silanols or alkoxides are protonated, so that better leaving groups $\left(\mathrm{H}_{2} \mathrm{O}\right.$ or $\left.\mathrm{ROH}\right)$ are present. Like for the acid-catalyzed hydrolysis, the reaction does not require a proton transfer in the transition state. The rate of siloxane bond ( $\mathrm{Si}-\mathrm{O}-\mathrm{Si}$ ) hydrolysis increases above $\mathrm{pH} 4$ and at very low $\mathrm{pH}$. Siloxane networks are formed under those conditions in which depolymerization is less likely to occur. In this situation, the condensation is irreversible and siloxane bonds cannot be hydrolyzed once they are formed. For example, by replacing water with a $90 \%$ methanol $10 \%$ water mixture, the solubility of silica is reduced by a factor of 28 and hydrolysis of siloxane bond is less likely. In Fig. 1.12, a schematic representation of hydrolysis and condensation reaction of silica, under acid or base catalysis, is reported. 
Acid catalysis<smiles>CC[Si](OC)(OC)[Si](OC)(OC)OC</smiles>

$\mathrm{H}_{2} \mathrm{O}$
Base catalysis

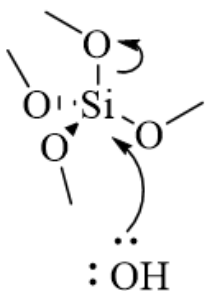

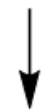
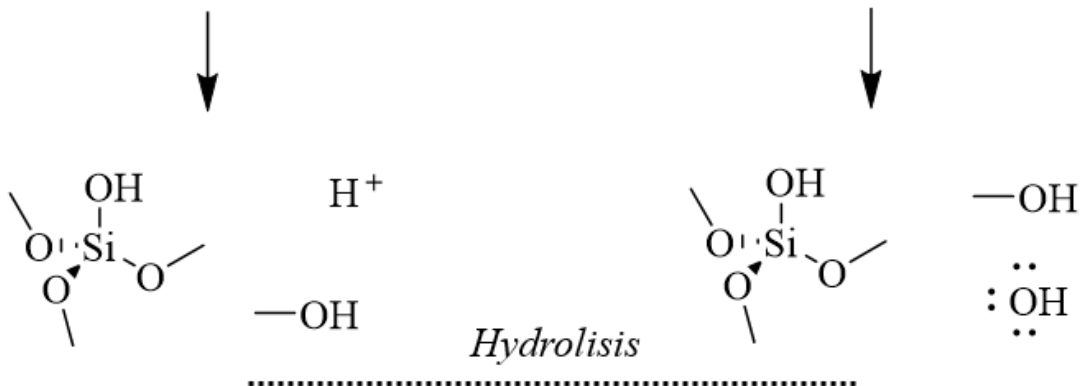

\section{Condensation}<smiles>O[Si](O)(O)C[Si](O)(O)O</smiles><smiles>O[Si](O)(O)[Ge]#[W]CCC[Si](O)(O)(O)(O)O</smiles><smiles>O[Si](O)(O)O[Si](O)(O)O</smiles>

- Particle growth

- Aggregation
$\mathrm{H}_{3} \mathrm{O}^{+}$<smiles>O[Si](O)(O)O[Si](O)(O)O</smiles>

- Ostwald ripening

- Particle growth

Figure 1.12: Schematic representation of hydrolysis/condensation reactions of tetramethyl ortosilicate (TMOS) under acid and base catalysis. 
It has been observed that acid catalyzed sol-gel processes lead to the formation of bigger particles with less defects, while base catalyzed syntheses lead to the formation of smaller particles featuring more defects.

The most implemented precursors for sol-gel synthesis of silica are tetraethyl ortolisicate (TEOS) and tetremethyl ortosilicate (TMOS). Bernards et al. studied the hydrolizability of siloxane featuring alkoxy residues of different length. They found that the hydrolyzability of the silanes in an acidic environment was inversely proportional to the size of the alkoxy residues (TMOS > TEOS > TPOS, Fig. 1.13). They explained that the effect of the alcohols on the hydrolysis rate of each of the silanes was due to differences in degree of dissociation of $\mathrm{HCl}$ in each one of the alcohols (methanol > ethanol, 1-propanol > 2propanol).$^{60}$

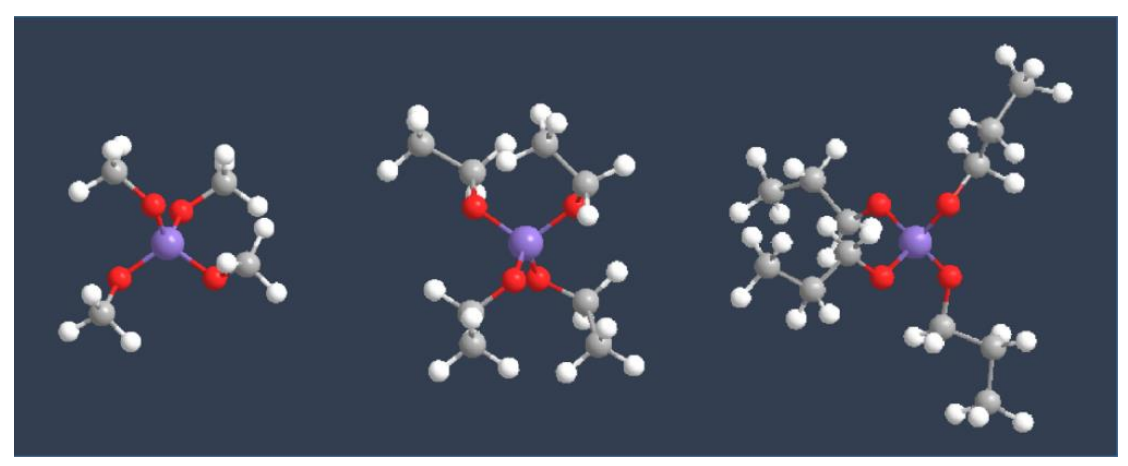

Figure 1.13: Ball and stick representation of tetramethyl ortosilicate (TMOS), tetraethyl ortosilicate (TEOS) and tetrapropyl ortosilicate (TPOS).

\subsubsection{Fluoride catalyzed hydrolysis and condensation}

Besides acid and base catalysis, there is a less common way to catalyze sol-gel reactions. $\mathrm{F}^{-}$ions have been reported to efficiently catalyze hydrolysis/condensation of alkoxy silanes decades ago. ${ }^{61}$ Hydrolysis/condensation reactions follow the pathways reported in Fig. 1.14. In the hydrolysis reaction, a nucleophilic fluorine ion approaches a molecule of alkoxy silane in solution. $\mathrm{A} \mathrm{S}_{\mathrm{N}} 2$ reaction occurs, forming a partially fluorinated silicon alkoxide that in presence of $\mathrm{H}_{3} \mathrm{O}^{+}$, yields water and alcohol as by-products. Due to strong inductive effect, fluorine withdraws electron density from the silicon atom, making it more susceptible to nucleophilic attack from $\mathrm{H}_{2} \mathrm{O}$. Consequentially, a pentacovalent complex is formed in the presence of water. The complex reacts to form a partially hydrated silicon alkoxide and hydrofluoric acid. The condensation process occurs by formation of a hexacovalent intermediate. Iler postulated that the effectiveness of fluorine in the polymerization reaction is due to the smaller ionic radius of the fluorine anion, compared to that of the hydroxyl. 
A

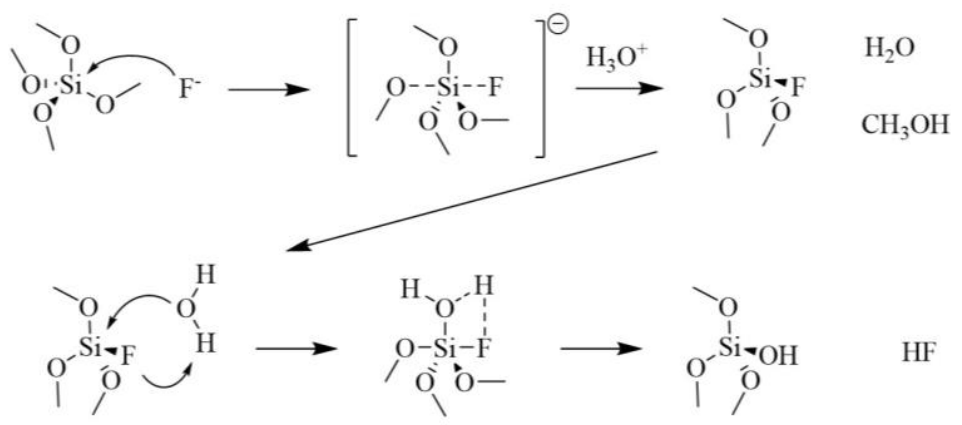

B

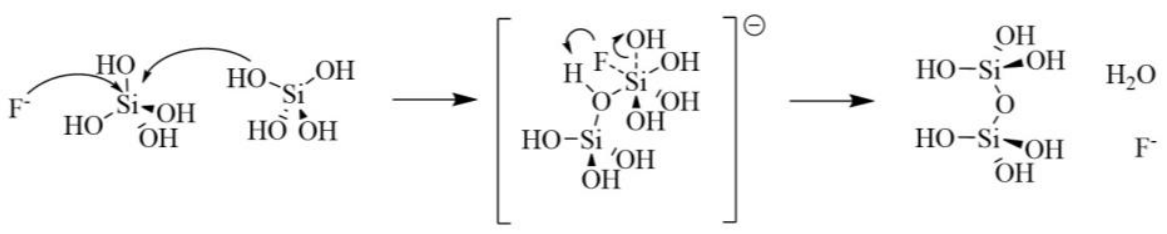

Figure 1.14: Fluoride-catalyzed hydrolysis (A) and condensation (B) of tetramethyl ortosilicate. Adapted from ref. 62.

Other anions, such as $\mathrm{Cl}^{-}, \mathrm{Br}^{-}, \mathrm{I}^{-}, \mathrm{NO}_{3}{ }^{-}, \mathrm{SO}_{4}{ }^{-}$are all bulkier than hydroxyl and for that, are much less effective than hydroxyl in catalyzing these reactions by nucleophilic attack. ${ }^{62}$ Under optimal conditions, fluoride catalyzed sol-gel synthesis can lead to mesoporous materials featuring high surface area and relatively narrow pore size distribution.

Reale et al. studied the influence of $\mathrm{Si} / \mathrm{F}^{-}$ratio on the textural properties of pure silica materials. For that, they used $\mathrm{NH}_{4} \mathrm{~F}$ as fluoride source. $\mathrm{NH}_{4}{ }^{+} / \mathrm{NH}_{3}$ equilibrium in water ensures the presence of protons in the reaction medium, needed for the mechanism described in Fig. 1.14. Varying the $\mathrm{Si} / \mathrm{F}^{-}$ratio between 15 and 320, they were able to obtain materials featuring specific surface area values (BET) ranging from 500 to $1000 \mathrm{~m}^{2} / \mathrm{g}$ and mean pore size $(\mathrm{BJH})$ ranging between 75 and $30 \AA$. The authors explained these variations by considering that the larger the mesoporous diameter, the larger the average silica particle radius. This could denote that the nucleation of silica particles is highly disfavored at high fluoride concentration, which would result in a low concentration of viable nuclei that can grow at the expenses of the silica precursor, in solution. Accordingly, high fluoride concentration would promote the formation silica particles featuring large diameter and large interparticle void.

The authors also studied the effect of the alcohol used as co-solvent by preparing materials using a 1:1 mixture of water and one among methanol, ethanol and 2-propanol. Upon increase of the alcohol size, surface area decreased and mean pore size increased. 
Nucleophilic attack of water to the fluorinated silicon intermediate, which is the rate determining step in the TMOS hydrolysis, is less favored by more polar solvents. Consequentially, a lower amount of nuclei is formed as consequence of the slower hydrolysis kinetics. Coherently, the silica material prepared in 2-butanol resembles that prepared using the highest fluoride concentration. ${ }^{63}$

It is important to highlight that these syntheses are carried out at neutral $\mathrm{pH}, 36^{\circ} \mathrm{C}$, using low amount of solvent and in the absence of templating agents. By tuning the composition of the synthesis mixture, it is possible to achieve highly porous materials, with textural properties that are tuned for a specific application. Moreover, $\mathrm{F}^{-}$sol-gel syntheses allow to incorporate $\mathrm{Al}$ and $\mathrm{Ti}$ within the network as well as organic building blocks, by substituting a fraction of TMOS with an equimolar amount of either an $\mathrm{Al}$ (or Ti) precursor or a silyl-derivative of the type $\mathrm{RSi}\left(\mathrm{OR}^{\prime}\right)_{3}$, baring a functional group $\mathrm{R}$ of interest.

\subsubsection{Surfactant mediated sol-gel synthesis}

As mentioned before, surfactants are widely implemented in sol-gel synthesis to achieve mesoporous silica materials, for applications in several fields. Supramolecular aggregates of ionic surfactants (long-chain alkyltrimethylammonium halides) and non-ionic triblock copolymers have been used as structure-directing agents (SDAs) in many synthetic procedures. In solution, under certain conditions, they form of a lyotropic liquidcrystalline phase, which can guide the assembly of ordered mesostructured materials through hydrolysis and condensation of the silica precursors under basic conditions. The mesoporous materials are obtained by removal of the surfactant by extraction or calcination.

Typically, a lyotropic liquid-crystalline phase is formed above a certain concentration of surfactant. However, a cooperative self-assembly between the SDA and the silica precursors can lead to a liquid-crystal phase with hexagonal, cubic, or laminar arrangement, even at lower concentration of surfactant. An essential condition to ensure this cooperative self-assembly and avoid phase separation is the presence of attractive interactions between the template and the silica precursor. Such interactions vary, depending on the type of surfactant and on the $\mathrm{pH}$ of the solution. If the reaction takes place under basic conditions, silica species will be present in the form of anions. If a cationic quaternary ammonium surfactant is used as the SDA, electrostatic interactions such as those depicted in Fig 1.15 A will be produced. However, if the preparation takes place below $\mathrm{pH}$ 2 , silica species will be positively charged and to produce an interaction with the cationic surfactant, it is necessary to add a counter ion $\mathrm{X}^{-}$(usually a halide, Fig. 1.15 B). Although less commonly, negatively charged surfactants (e.g. long-chain alkyl phosphates) can also be used as the SDA. Compared to positively charged surfactant, a reverse situation is expected, with protonated silicon species interacting with the negatively charged functional groups of the surfactant molecules, at $\mathrm{pH}$ below 2 (Fig. 1.15 C), and a mediating positive ion $\mathrm{M}^{+}$needed under basic conditions (Fig. 1.15D). Lastly, when non-ionic surfactants are used (e.g. long chain amines), attractive interaction between SDA molecules 
and silica precursors are provided by hydrogen bonds. Such interactions can be promoted by adding to the mixture either polar molecules (e.g. polyethylene oxide, depicted as triangles in Fig. 1.15E) or ion pairs (Fig. 1.15F). ${ }^{49}$
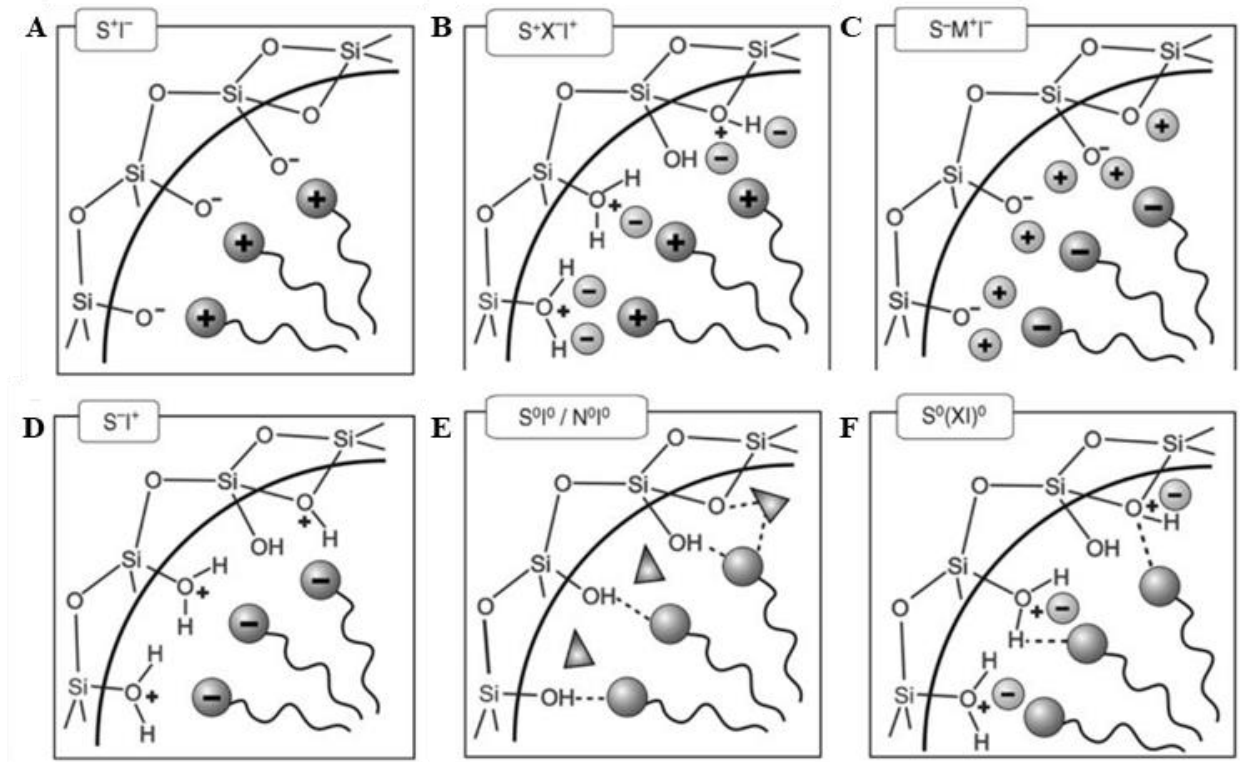

Figure 1.15: Schematic representation of the possible interactions between silica precursors and surfactant molecules. Adapted from ref. 49.

\subsubsection{Handling silyl-derivatives}

Throughout the first part of this Chapter, many silyl-derivatives of different complexity were shown. Considering that most of the research work reported in this Thesis relies on the uses of precursors of the type $\mathrm{RSi}\left(\mathrm{OR}^{\prime}\right)_{3}$, it is worth to briefly discuss these class of molecules. Alkoxy groups are highly reactive species. They can easily undergo partial hydrolysis and condensation leading to oligomers. This is particularly problematic considering that, in many cases, they are used as precursors of materials in which the functional groups R need to be homogeneously dispersed throughout the material (heterogeneous catalysts, adsorbents, sensors etc.). Silyl-derivatives are very moisture-sensitive. For that, the design of synthetic routes usually aims to incorporate the alkoxy-silyl group in the last step of the preparation of the building block. Whenever this is not possible, silyl-derivatives need to be protected from humidity, by storing them under inert atmos- 
phere and by always carrying out reactions that involve them under anhydrous conditions, especially if acid or bases are also present in the reaction mixture. Additional care needs to be taken in the work-up procedure, as distillation at high temperature can cause spontaneous condensation of alkoxy groups. The size of the alkoxy group also affects the kinetic of hydrolysis with TMOS containing synthesis mixture, reaching the sol-gel transition after shorter time, compared to TEOS-based mixtures. This is in line with what can be deduced by observing the mechanism depicted in Fig. 1.12 and the ball and stick structures depicted in Fig. 1.13. Ethoxy substituent are bulkier than methoxy and therefore they partially hinder the approach of the nucleophile to the silicon. ${ }^{58}$ Consequentially, methoxy-silyl groups are expected to be less stable and more reactive than ethoxysilyl groups. 


\subsection{Silica-based hybrids in heterogeneous catalysis}

With the aim of highlighting the broad scope of application of silica-based hybrid catalysts, a general overview of different type of active sites bounded onto silica supports is described in this section, together with few representative examples of their synthesis and applications.

\subsubsection{Acid catalysts}

Many reactions in the chemical industry are carried out under acid catalysis conditions. Purely inorganic microporous zeolites are perhaps the most notable example of heterogeneous catalysts, being them implemented in several chemical processes. ${ }^{64}$ Nevertheless, the small pore size limits their application to certain reactions, while others require catalysts in which the active sites are accessible to more bulky molecules. One solution to this problem is functionalizing mesoporous silica supports with molecules baring Brönsted acid groups. ${ }^{65}$ Heteropolyacids (HPAs) are polyoxometalates constituted by $\mathrm{MO}_{\mathrm{x}}$ polyhedra (usually distorted octahedral) which surround one or more heteroatoms (Fig. 1.16 A). ${ }^{66}$ Several authors reported on silica-supported HPAs for applications in heterogeneous catalysis. Sato et al. supported three types of HPA over differently porous silica by wet impregnation: phosphotungstic acid $\left(\mathrm{H}_{3} \mathrm{PW}_{12} \mathrm{O}_{40} \cdot n \mathrm{H}_{2} \mathrm{O}\right)$, silico-tungstic acid $\left(\mathrm{H}_{4} \mathrm{SiW}_{12} \mathrm{O}_{40} \cdot 24 \mathrm{H}_{2} \mathrm{O}\right)$, and phospho-molybdic acid $\left(\mathrm{H}_{3} \mathrm{PMo}_{12} \mathrm{O}_{40} \cdot n \mathrm{H}_{2} \mathrm{O}\right)$. The catalysts were used in the vapor-phase dehydration of glycerol to acrolein. It was found that silico-tungstic acid, supported on silica materials featuring mesopores of $10 \mathrm{~nm}$, showed the best catalytic activity, with 98 mol\% conversion and an acrolein selectivity higher than $85 \mathrm{~mol} \%$. Moreover, they observed an influence of the meropores size on the catalytic activity, with a very steep deactivation of the catalysts when the HPA were supported onto a silica material having mesopores of $3 \mathrm{~nm} .{ }^{67}$ In another study, Blanco et al. used silica supported $\mathrm{H}_{3} \mathrm{PMo}_{12} \mathrm{O}_{40} \cdot n \mathrm{H}_{2} \mathrm{O}$ and $\mathrm{H}_{3} \mathrm{PW}_{12} \mathrm{O}_{40} \cdot n \mathrm{H}_{2} \mathrm{O}$ for dehydration reactions of different alcohols. Specifically, the dehydration of 1,2-diphenylethanol, 1-3,4dimethoxyphenyl-2-phenylethanol and cholesterol was investigated. The authors found both catalysts to be highly active and selective with no appreciable loss of the catalytic activity over multiple cycles. ${ }^{68}$ Laurenti et al. prepared silica supported $\mathrm{H}_{4} \mathrm{SiW}_{12} \mathrm{O}_{40}$. $n \mathrm{H}_{2} \mathrm{O}$ and $\mathrm{H}_{3} \mathrm{PW}_{12} \mathrm{O}_{40} \cdot n \mathrm{H}_{2} \mathrm{O}$ by wet impregnation and tested them for the alkylation of 3-methylthiophene with 2-methyl-2-butene, used as model reaction for gasoline desulfurization. Their performances were shown to be superior to those of zeolites USY, Beta and MCM-22. Supported $\mathrm{H}_{3} \mathrm{PW}_{12} \mathrm{O}_{40} \cdot n \mathrm{H}_{2} \mathrm{O}$ was also tested in a real feed of FCC gasoline with promising results. ${ }^{69}$

Another class of silica-supported acid catalysts are based on the incorporation of polyphosphoric acid (PPA-SiO ${ }_{2}$ ) that has been used as a protic acid catalyst for organic reactions. PPA is a viscous liquid at room temperature, which makes its handling challenging for scaled-up applications. ${ }^{70}$ To overcome this limitation, supporting PPA onto silica has been proposed and studies have been reported for a number of catalytic applications. For example, Aoyama et al. reported on PPA-SiO 2 for the conversion of carbonyl compounds 
into oxathioacetals and dithioacetals. Under mild conditions (room temperature), they were able to achieve high yields in reactions involving a number of carbonyl compound and either mercaptoethanol or 1,2-ethanedithiol. ${ }^{70}$ In another study, Maleki et al. prepared a magnetic silica-supported acid catalyst, $\mathrm{NiFe}_{2} \mathrm{O}_{4} @ \mathrm{SiO}_{2}-\mathrm{PPA}$, for the synthesis of $N, N^{\prime}$-alkylidene bis-amide derivatives by one-pot condensation of aldehydes and amides (Fig. 1.16 B). NiFe $\mathrm{O}_{4} @ \mathrm{SiO}_{2}-\mathrm{PPA}$ was found to be an efficient catalyst for the reaction of aldehydes with amides, allowing to achieve the corresponding product in yields ranging from high to excellent. Notably, besides the typical advantages of supported catalysts over non-supported ones, such as not being corrosive and presenting lower disposal problems, this magnetic catalyst could be easily isolated from the reaction mixture by simple magnetic separation, and it could be used in six reaction cycles without any significant loss of activity. ${ }^{71}$ Other inorganic acids such as perchloric acid, ${ }^{72}$ fluoroboric $\mathrm{acid}^{73}$ and sulfuric acid ${ }^{74}$ have been supported onto silica. Although effective in overcoming some of the limitations of microporous acid catalysts, these purely inorganic materials present little margin for modifications and fine tuning of their properties for a specific catalyzed reaction.

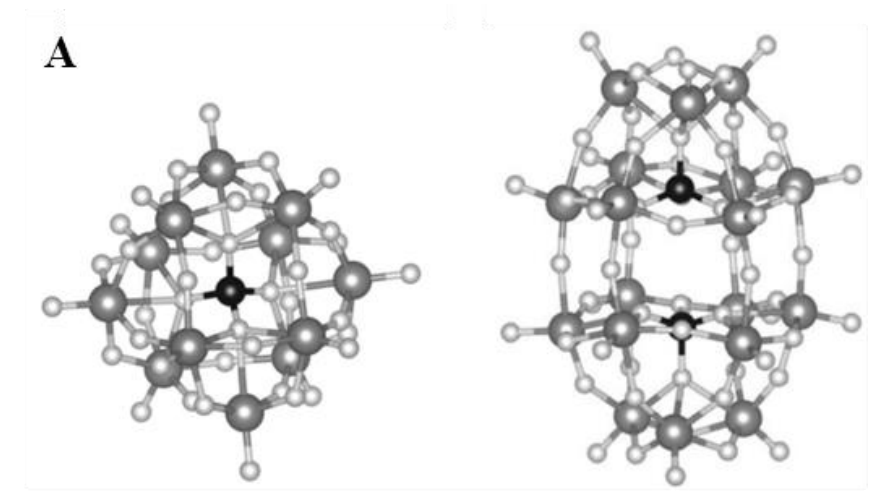

B<smiles>NC(=O)c1ccccc1</smiles>

Figure 1.16 A: Ball and stick representations of two of the most common HPA variants. $P$ (black), Mo (dark grey), $O$ (light grey). Adapted from ref. $66 \mathrm{~B}$ : Model reaction for synthesis of $N, N^{\prime}$-(3-nitroophenylmethylene)dibenzamide. ${ }^{71}$ 
Perhaps, the most versatile way to incorporate strong Brönsted acid sites into mesoporous silica matrixes is the synthesis of supported sulfonic acid groups. The nature of the acidic residues allows for a fine tuning of the acidity of the sulfonic proton by exploiting the versatility of organic synthesis. ${ }^{75}$ The acidity is strongly influenced by the chemical makeup of the sulfonic groups: sulfonic groups were found to be more acidic, for example, when attached to an aromatic ring or to a fluorinated carbon atom (Fig. 1.17). A detailed introduction on the state of the art of silica-supported sulfonic acid groups will be reported at the beginning of Chapter 3 .
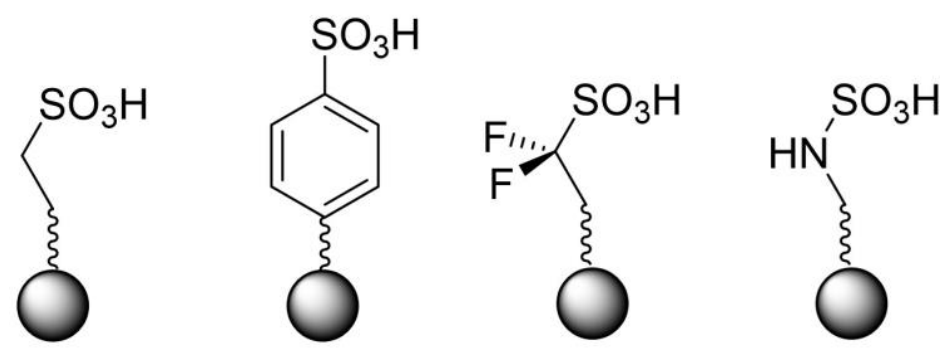

Figure 1.17: Schematic representation of some of the different chemical makeups of sulfonic acid groups. Adapted from ref. 75.

\subsubsection{Base catalyst}

Another important class of catalysts are those carrying base functionalities, able to activate certain functional groups by electron-donation. Within the realm of silica-supported hybrids, the majority of these catalysts consist of N-based compounds (amines and Ncycles of various nature). Brunel et al. compared the catalytic activities of primary and tertiary amino groups bounded to MCM-41 in the Knoevenagel condensation ${ }^{76}$. Bigi et $a l$. indicated a strong correlation between the activity scale of supported amines and that of the corresponding homogeneous counterparts, the order of activity being primary > secondary $>$ tertiary in nitroaldol condensation reactions at $90{ }^{\circ} \mathrm{C} .{ }^{77}$ However, this general trend has not always been confirmed. For example, Kitayama et al. reported on mesoporous silica FSM-16 functionalized with secondary amines being more active for the aldol reaction than FSM-16 functionalized with primary amines. ${ }^{78}$ In another study, Katz et al. found that acid-base synergy between surface silanols and primary amines could also affect chemical reactivity and that such cooperativity enhanced the selectivity to unsaturated products in base-catalyzed reactions, such as the Michael, Henry and Knoevenagel reactions. ${ }^{79}$

Shantz et al. prepared a series of surface-anchored amines by either grafting or tethering reactions and compared their activity in the Henry reaction between nitrobenzaldehyde and nitro-methane (Fig. 1.18 A). The authors found the primary amine (catalyst 1 ) to be 
the less active than secondary and tertiary amine. They also observed differences in selectivity. The primary amine appeared to follow the imine formation mechanism, favoring the formation of the nitrostyrene product. On the other hand, the samples containing secondary amines resulted to be highly selective to the nitroalcohol product. Interestingly, the selectivity to the nitroalcohol product increased proportional to the amine loading. Among the secondary amines, the less sterically hindered one (catalyst 2) appeared to be the most active. This study emphasizes how crucial it is to choose a specific type of amine for a specific reaction, keeping into account basic strength as well as reaction mechanisms, depending on the product of interest.

Wang and co-workers found that silanols-capped hybrids baring amine groups were much less active than non-capped samples. ${ }^{77}$ Silica supported guanidine moieties have been synthesized by several authors and implemented in a number of catalytic reactions. Dupont et al. synthetized a guanidine-based silyl-derivative by reacting 3-aminopropyltrimethoxysilane and dicyclohexylcarbodiimide (Fig. $1.18 \mathrm{~B}$ ). The silyl-derivative was grafted onto silica and the hybrid was tested for the production of biodiesel. Conversions up to $98 \%$ were obtained for soybean oil methanolysis at $353 \mathrm{~K}$ for $3 \mathrm{~h}$. The catalytic system was transposed to a semi-pilot scale in a continuous flow reactor. ${ }^{80} \mathrm{In}$ another study, Sartori et al. used a guanidine-based hybrid catalyst in the cycloaddition of epoxides, to produce cyclic carbonates at $140{ }^{\circ} \mathrm{C}$. Different epoxides were used, with yield at $70 \mathrm{~h}$ ranging between 55 and $95 \%$. The catalyst was less active than the homogeneous counterpart, although it could be reused over three catalytic cycles. ${ }^{81}$

Another relevant class of base hybrid catalysts are supported imidazolium based moieties. In an exemplificative study, Hesemann et al. developed a mesoporous SBA-15 type silica, bearing ionic imidazolium substructures, via post-synthesis grafting reactions using bi-silylated imidazolium precursors (Fig. 1.18 C). Passivated versions of the hybrids were prepared and the four catalysts were tested in Henry reactions and cycloadditions of carbon dioxide to epichlorohydrin. In both types of reaction, the passivated versions showed higher catalytic activity, although to a lesser extent in the case of cycloaddition reactions. The authors speculated a beneficial synergistic effect involving the imidazolium cation, its counter-ion and the hydrophobic groups introduced during the passivation of the material..$^{82}$ Gianotti et al. prepared a hybrid catalyst using proton sponges as organic building blocks. The proton sponges, i.e. diamines that exhibit very high basicity, were reacted to obtain bi-silylated building blocks (Fig. 1.18 E). The resulting precursors were co-condensed with TMOS under fluoride catalyzed conditions. The hybrid was tested in the Knoevenagel condensation between benzaldehyde and ethyl cyanoacetate, showing high activity and recyclability over four catalytic cycles. ${ }^{83}$ 


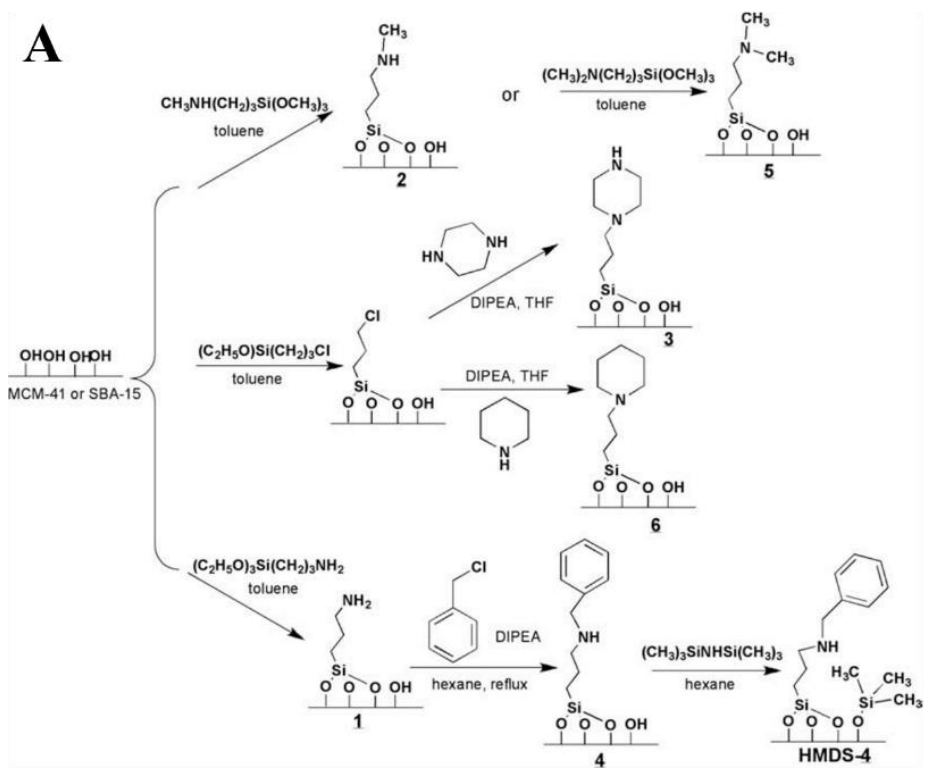

B

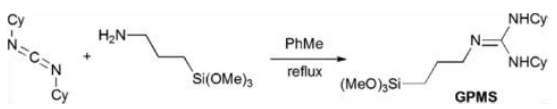

D

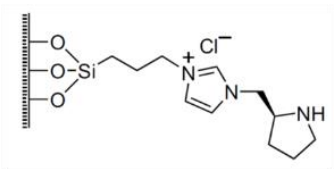

$\mathbf{F}$

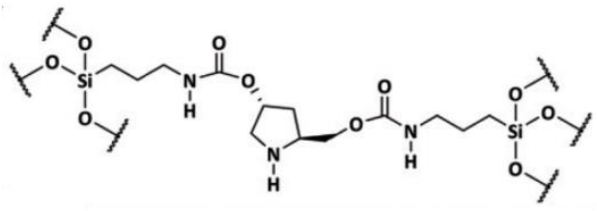

C
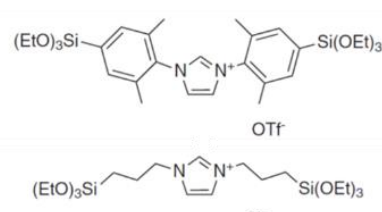

$\mathrm{Cl}$

$\mathbf{E}$

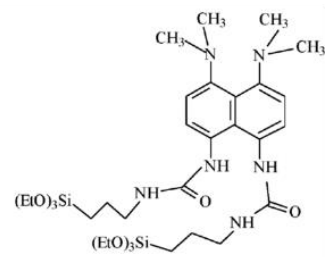

Figure 1.18 A: Schematic representation of the grafted amines reported in ref. ${ }^{77}$ B: Synthesis of guanidine-based silyl-derivative.$^{80} \mathrm{C}$ : Imidazolium-based silyl-derivatives. ${ }^{82} \mathrm{D}$ : Silica-supported pyrrolidine-based chiral ionic liquid. ${ }^{84} \mathrm{E}$ : 1,8-bis(dimethylamino)naphthalene (DMAN)-based silyl-derivative. ${ }^{83} \mathrm{~F}$ : Silica-functionalized pyrrolidine-based chiral hybrid catalyst. ${ }^{85}$ 
The capacity for electron rich atoms, such as $\mathrm{N}$, to coordinate electrophilic atoms, together with the versatility of organic syntheses, allows for the possibility of functionalizing silica supports with chiral amines to carry out asymmetric catalytic reactions. Wang et al. developed a silica supported pyrrolidine-based chiral ionic liquid capable of catalyzing Michael addition reaction of ketones with nitrostyrenes in good yields, excellent enantioselectivities and high diastereoselectivities. The catalyst could be reused up to six times (Fig. 1.18 D) ${ }^{84}$ In another study, Llopis et al. reported on a new chiral mesoporous hybrid material based on pyrrolidine units integrated into an organic-inorganic structure, obtained from a bi-silylated precursor. The mesoporous hybrid was prepared by fluoride sol-gel methodology under soft synthesis conditions and in the absence of structural directing agents. The chiral solid was tested as asymmetric catalyst in the Michael addition reaction between a wide range of linear aldehydes to nitroalkenes with excellent yields as well as high enantioselectivities. The hybrid catalyst was stable, easily separable and could be reused several times (Fig. 1.18 F). ${ }^{85}$

\subsubsection{Organometallic complexes}

The possibility of covalently incorporate organic moieties within inorganic insoluble structures, allowed several research groups to heterogenize organometallic complexes. This is perhaps one of the most promising area of research within the field of hybrid catalysts preparation, as transition metal complexes can catalyze a number of reactions including C-C, C-N, C-O bonding formations, oxidations, reductions, as well as different kind of asymmetric reactions. In this Section, relevant examples involving silica-supported complexes of different metals are shown, with the scope of describing common approaches implemented for supporting organometallic complexes onto silica. In Chapter 5 , studies related to the classes of complexes dealt with in this Thesis will be reported in detail.

Suzuki-Miyaura, Heck and Sonogashira coupling reactions are among the most efficient routes for the formation of $\mathrm{C}-\mathrm{C}$ bonds. Palladium catalysts have been long known to be highly active in these coupling reactions and several types of ligands have been used to support palladium onto silica matrixes ${ }^{86}$ Luo et al. synthesized a mesoporous silica-supported palladium imino-pyridine complex by implementing a multi-step strategy. Firstly, they carried out a nucleophilic substitution reaction between imino-pyridine ligand and (3-chloropropyl)triethoxysilane, achieving a silyl-derivative with coordination capacity. Secondly, they grafted the silyl-derivative onto MCM-41. Lastly, after quantifying the amount of ligand in the solid, they obtained the supported complex by suspending the solid in a palladium acetate solution in acetone, at room temperature (Fig. 1.19). 

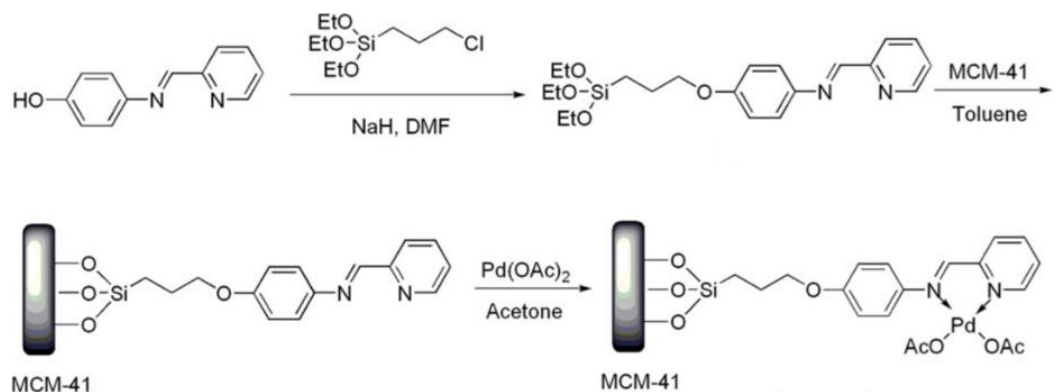

Figure 1.19: Schematic representation of the preparation of MCM-41 supported Pd complex. ${ }^{87}$

The catalyst was found to be an efficient for Suzuki-Miyaura coupling reactions in aqueous medium. The reactions of various aryl bromides with arylboronic acids were carried out, using low Pd loading in the reaction mixture $(0.1 \mathrm{~mol} \%)$, with good to excellent yields and very low Pd leaching $(<0.2 \mathrm{ppm}){ }^{87}$

Sanchez et al. prepared mesoporous silica-supported chiral ruthenium NHC-based catalysts for asymmetric reactions. Neutral unsymmetrical pyridine pincer-type ligands with lateral (S)-prolinamide donor function and a $\mathrm{N}$-heterocyclic carbene (NHC) moiety were prepared. The prolinamide substituents of the $\mathrm{CNN}$-pincer backbone were functionalized with a triethoxysilyl group. The incorporation of ruthenium was achieved by Lin's method of transmetalation from intermediate silver (I) complexes. Lastly, the silyl-derivative $\mathrm{Ru}$ complexes were grafted by heating at reflux in toluene for $24 \mathrm{~h}$ (Fig. 1.20). The heterogenized catalysts were observed to be highly active, selective, and recyclable in the asymmetric hydrogenation of alkenes and showed promising results in the cyclopropanation of styrenes. ${ }^{88}$ 
<smiles></smiles>

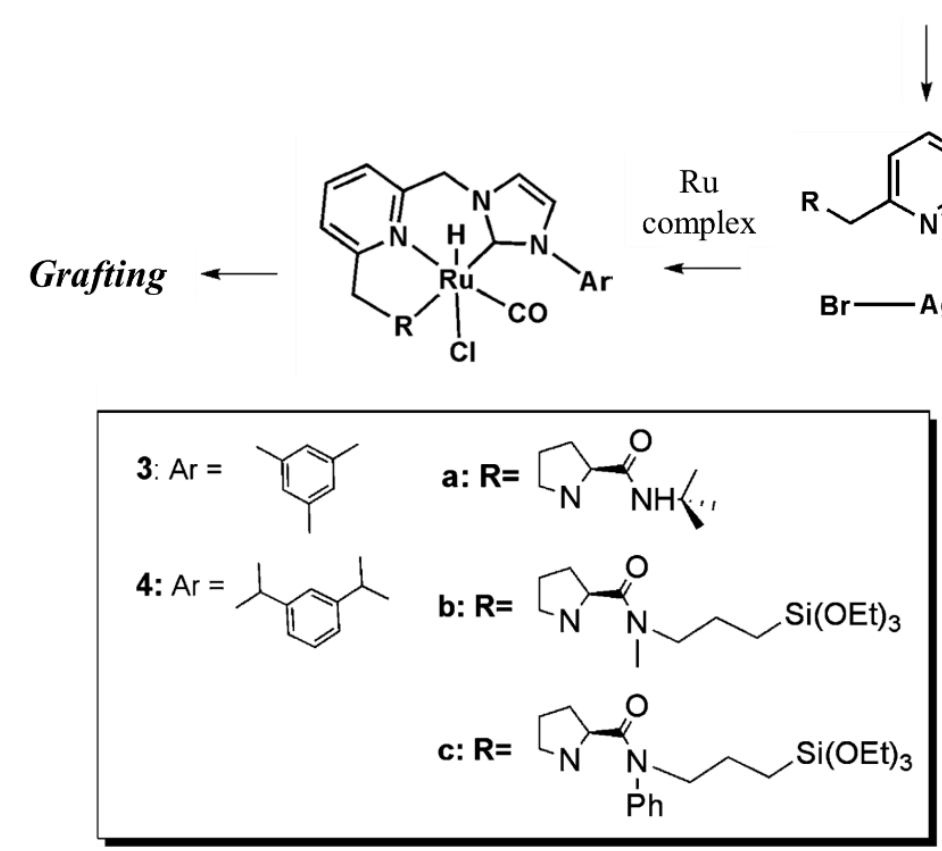

Figure 1.20: Schematic representation of the synthesis of silica-supported chiral Ru complexes. Adapted from ref. 88.

Corma et al. reported on the synthesis of periodic mesoporous organosilicas prepared using a bi-silylated vanadyl Schiff base complexes as building block. The synthesis of the catalysts was carried out following the scheme reported in Fig. 1.21 A. Firstly, a Suzuki coupling between p-vinylphenylboronic acid and 4-bromosalicylaldehyde derivatives was carried out (step b), followed by the diimine formation using ethylenediamine or (1R,2R)-(-)-1,2-diaminocyclohexane (step c). Subsequently, the formation of the vanadyl complex was performed (step d). The authors reported that an alternative strategy in which vanadyl complexation was carried out after the introduction of the trimethoxysilane groups on the ligand led to extensive hydrolysis and the formation of 
untreatable mixtures. Then, radical coupling between 3-mercaptopropyl trimethoxysilane and the p-styryl residues was performed, to achieve the bi-silylated vanadyl complex. Lastly, the organic building blocks were co-condensed with TEOS, in different proportions, to obtain the hybrid PMOs. The templating agent was removed by extraction.

A
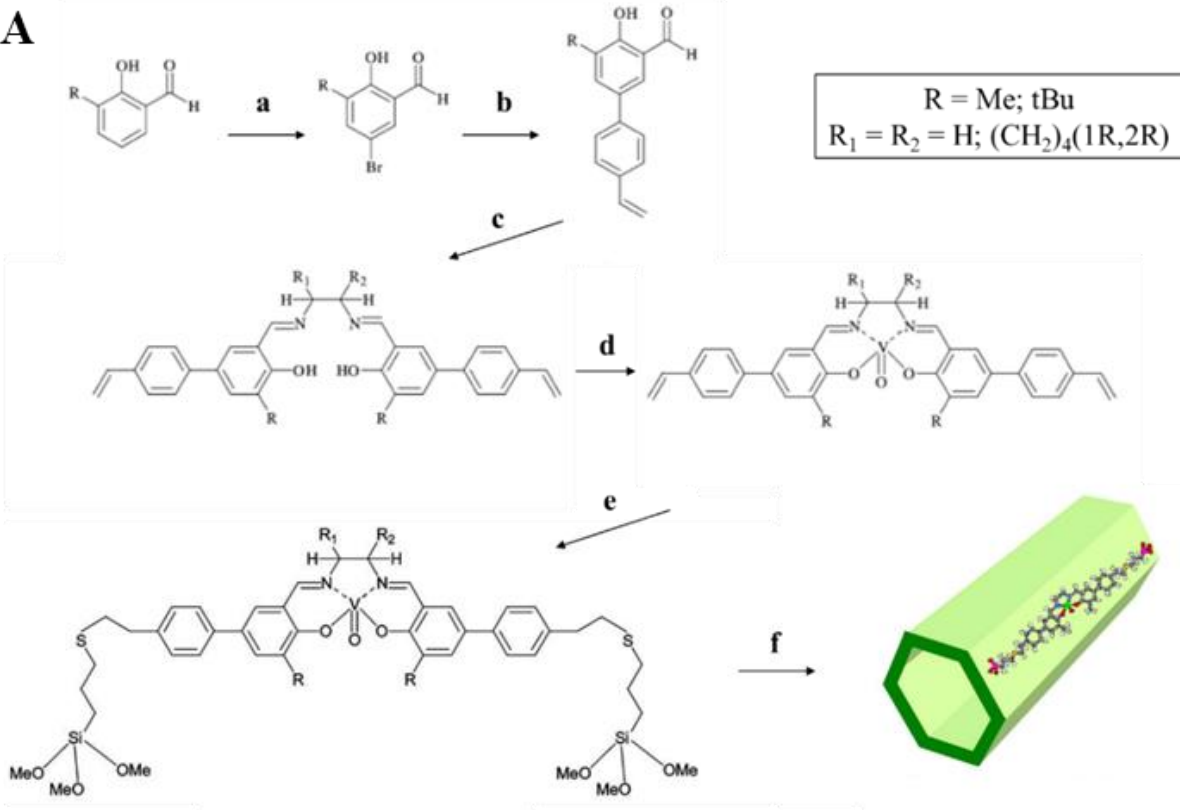

B

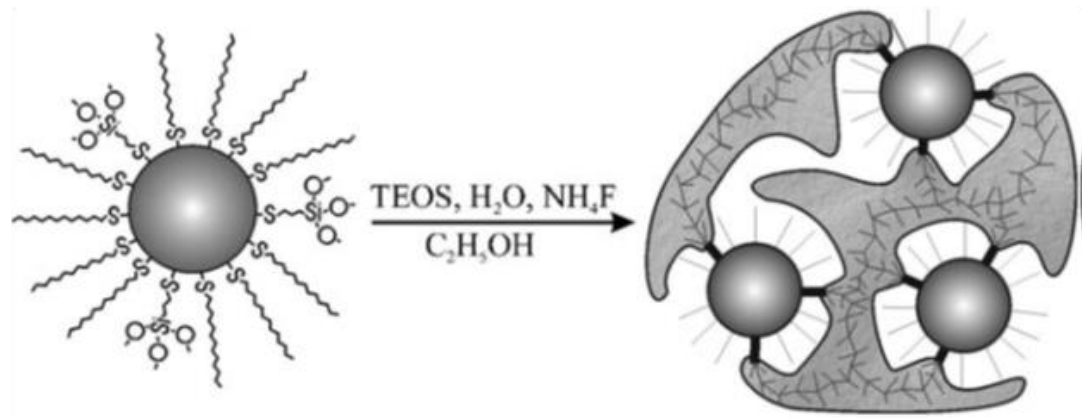

Figure 1.21 A: Schematic representation of the synthesis of PMOs containing vanadyl Schiff base complexes. Adapted from ref. 89. B: Schematic representation of the synthesis of gold NPs imbedded into silica. ${ }^{90}$ 
The catalysts were tested in the room-temperature cyanosilylation of aldehydes in chloroform, using trimethylsilyl cyanide as reagent, showing high conversions and complete selectivities to the desired silylated cyanohydrins. Although ee of $30 \%$ was observed for the cyanosilylation of benzaldehydes, the result was much lower than that observed for the same reaction performed using the vanadyl complex in homogeneous phase. ${ }^{89,91}$

Organic-inorganic solids have also been cleverly implemented to incorporate metal nanoparticles within silica structures. ${ }^{92,93}$ In a representative example, Budroni et al. prepared Au nanoparticles embedded in silica. Firstly, ionic gold was reduced with hydrazine, in the presence of a mixture of 1-dodecanethiol and 3-mercaptopropyl trimethoxysilane (MPMS). Alkanethiols lowers the surface energy of the Au particles and consequently their tendency to aggregate. Alkanethiol-stabilized gold nanoparticles were then dispersed into an ethanol solution, together with TEOS. Hydrolysis and condensation of the alkoxysilyl- groups was carried out using $\mathrm{NH}_{4} \mathrm{~F}$ as catalysts. 1-dodecanethiol molecules act as physical spacers between the particles and the silica shell (Fig. $1.21 \mathrm{~B})$. The method proposes to include the nanoparticles into a shell of silica that will prevent them from aggregation at high temperature, at the same time without limiting the accessibility of the reactant to the catalytic sites. ${ }^{90}$ 


\subsection{Silica-based multi-functional hybrid catalysts}

The capability of incorporating active sites within solid structures, fixing them in specific regions of the material, opens up to the possibility of designing heterogeneous catalysts featuring multiple active sites, to carry out cascade or tandem reactions, using only one recoverable catalytic system. This is no trivial task, considering that catalytically active sites are, by definition, reactive functional groups and that different active sites can interact with each other (or annihilate) in the synthesis mixture preventing their homogeneous distribution or causing their mutual inactivation. To avoid this, clever synthetic strategies are in need. Approaches based on silica are particularly advantageous for this purpose, for various reasons. The versatility of sol-gel chemistry and the reactivity of the surface of silica materials make it possible to introduce different functionalities in subsequent steps (e.g. co-condensation and grafting). Silica supports are also generally cheap and easy to synthetize. Moreover, their thermal and mechanical stability, their high surface area, tunable pore size diameter and the ease with which their surface can be modified in a post-synthesis step (introducing additional functional groups or varying their hydrophilic/hydrophobic properties) are welcomed features for inorganic supports. In this section, representative examples describing some synthetic strategies used for preparing multi-functional silica-based heterogeneous catalysts are reported.

Gianotti et al. prepared bifunctional mesoporous hybrid materials, containing both acid groups and proton sponges. Co-condensation of TMOS, 3-mercaptopropyltriethoxysilane (MPTES) and bi-silylated 1,8-bis(dimethylamino)naphthalene (DMAN-2Sil), was carried out under $\mathrm{NH}_{4} \mathrm{~F}$ catalysis, at low temperature and neutral pH (Fig. 1.22 A). After the synthesis, in order to oxidize thiol groups to sulfonic acids, the hybrid was stirred in $30 \% \mathrm{H}_{2} \mathrm{O}_{2}$ solution. The authors also prepared ordered mesoporous organosilica featuring the same functional groups. They achieved that by implementing a surfactant-mediated one-pot co-condensation of MPTES, disilylated-DMAN and TEOS, in presence of hexadecylamine (HDA). Template removal was performed by ethanol extraction using a Soxhlet apparatus and the thiol groups were oxidized to sulfonic acid groups with a $\mathrm{H}_{2} \mathrm{O}_{2}$ solution. Lastly, the bi-functional hybrids were also prepared by multi-step synthesis (Fig 1.22 B). In this case, firstly, both ordered and non-ordered containing MPTES silicas were synthesized following a template route and a $\mathrm{NH}_{4} \mathrm{~F}$ co-condensation route at room temperature. Secondly, after extraction of the template and oxidation of the thiol groups to sulfonic acids, mono-silylated DMAN (DMAN-1Sil) was grafted both on ordered and non-ordered materials. The hybrid catalysts were tested in the one-pot cascade reaction involving an acid-catalyzed acetal hydrolysis followed by a base-catalyzed Knoevenagel or nitroaldol (Henry) reaction. Interestingly, non-ordered hybrids, prepared by $\mathrm{NH}_{4} \mathrm{~F}$ catalyzed route, showed superior catalytic activity, compared to the other hybrids. This was particularly true when a more demanding methylene compound (such as diethylmalonate or nitromethane) was used as substrate. The authors tentatively ascribed the enhanced activity to the higher structural flexibility of the non-ordered silica network and to the higher amount of silanol groups that can perform a cooperative electrophilic activation. 
A

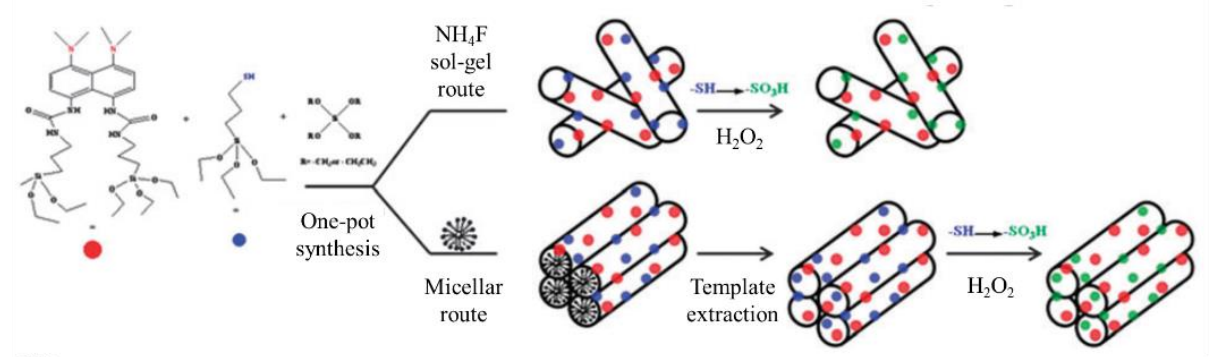

B

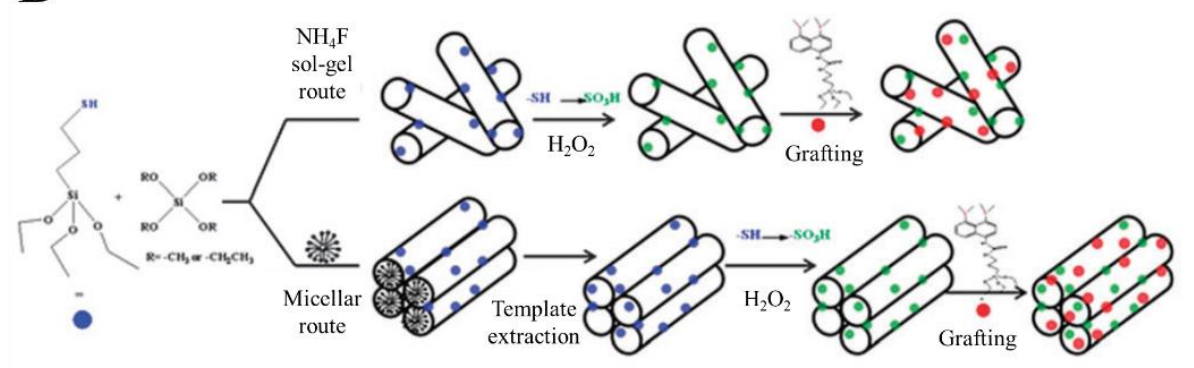

Figure 1.22 A: One-pot synthesis of bi-functional mesoporous hybrids. B: DMAN grafting on mesoporous silicas containing sulfonic acids. Adapted from ref. 94.

In another study, Asefa et al. prepared bi-functional mesoporous organosilicas baring Pd-diamino and propylamino residues. The synthesis was carried out following the scheme reported in Fig. 1.23, using MCM-41 and SBA-15 as supports. For both hybrids, firstly, 3-aminopropyltrimethoxysilane was grafted onto the silica support. Then, a silylfunctionalized Pd complex was prepared by mixing at room temperature $\mathrm{Pd}^{\mathrm{II}}$ acetate and $\mathrm{N}$-(2-aminoethyl)-3-aminopropyltrimethoxysilane (1:1 mol ratio). The silyl-derivative was then grafted onto the amino-functionalized mesoporous silica, in toluene, to achieve the bi-functional hybrid catalyst. The authors attributed great importance to using the appropriate solvent in each of the grafting procedures carried out. Specifically, by using isopropanol, they were able to achieve a more homogenous dispersion of the active sites throughout the surface of the support, at the same time leaving enough free silanol groups to perform a second grafting. For that, they used toluene, which is reportedly a good solvent for grafting processes. The hybrid catalysts were tested in the one-pot tandem Sonogashira-Henry reactions (Fig. 1.23, inset). The catalysts showed to be active in the tandem reaction with a $60 \%$ yield of the Sonogashira-Henry product, after $5 \mathrm{~h}^{95}$ 

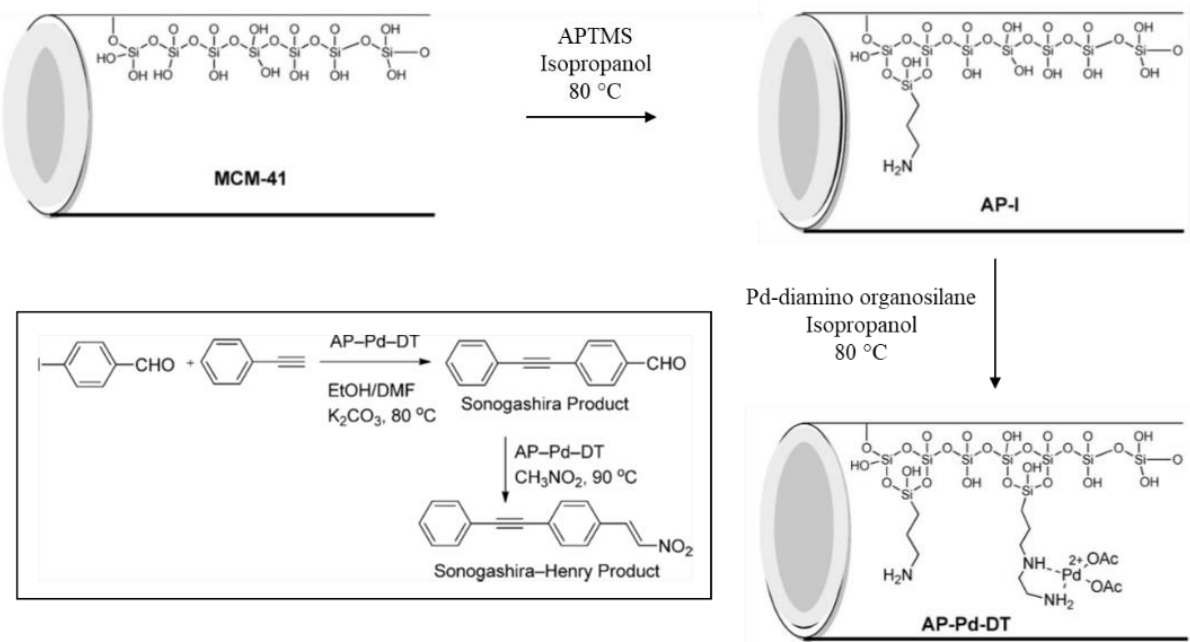

Figure 1.23: Schematic representation of the synthesis of bi-functional hybrid catalyst baring amino functionalities and Pd-diamino complexes. Inset: Sonogashira-Henry tandem reaction used for testing the activity of the hybrid catalyst. Adapted from ref. 95.

Another interesting possibility is the coexistence of acid/base functionalities together with metal nanoparticles, to perform tandem or cascade reactions. In an interesting study, Bäckvall et al. prepared an artificial metalloenzyme with two different modes of reactivity: one exhibited by a nanometallic component and one by an enzyme. The presence of the two functionalities in the same cavity of a mesoporous heterogeneous support created an environment in which these active sites could work in close proximity to one another in a cooperative fashion, to perform tandem catalysis. In this work, siliceous mesocellular foams (MCFs) were used as inorganic supports. MCFs are a class of porous siliceous materials featuring high pore volume and cavities, which make them the ideal platform for supporting macromolecules such as enzymes. The approach used for the multi-step synthesis was based on leaving the incorporation of the enzyme as the last one, as by doing otherwise the macromolecule would have risked to be affected by the strongly basic condition in which the metal nanoparticles could be obtained. An aminofunctionalized MCF was used as support for Pd nanoparticles deposition (1-2 nm in diameter) ${ }^{96}$ The material was then suspended in aqueous glutaraldehyde in phosphate buffer, to react the remaining amine groups, not interacting with the Pd. The enzyme Candida antarctica lipase B (CALB) was then reacted with the pendant aldehyde groups, to achieve the bi-functional catalyst (Fig. 1.24). The catalyst was tested in the dynamic kinetic resolution (DKR) of a primary amine. In this reaction a lipase enzyme selectively transforms only one enantiomer of the starting amine into the corresponding amide. 


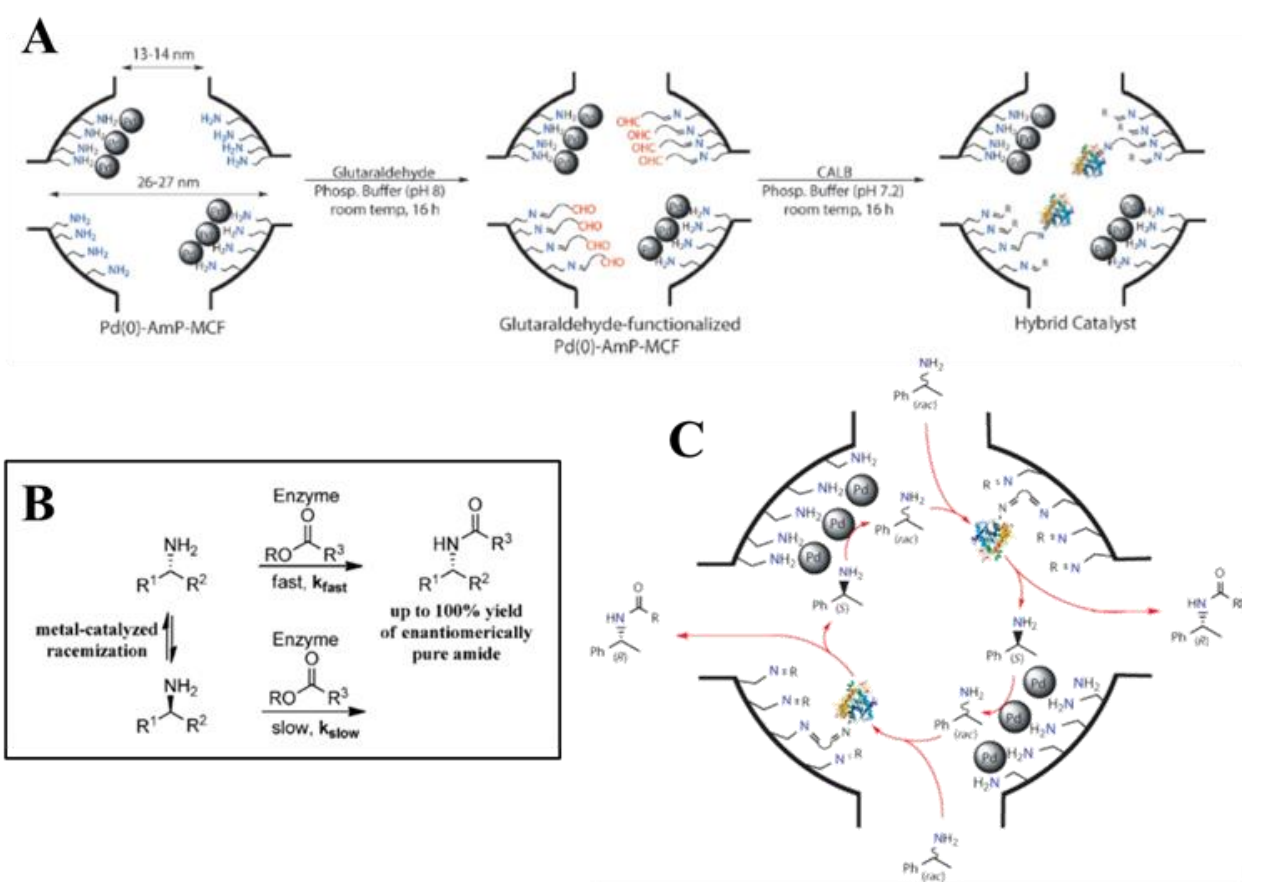

Figure 1.24 A: Synthesis of the bi-functional hybrid catalyst. B: Schematic representation of a general dynamic kinetic resolution (DKR) of a primary amine. C: DKR of an amine with a bifunctional biomimetic catalyst in which Pd nanoparticles and a lipase (CALB) are co-immobilized in the same pore of MCF. ${ }^{96}$

The racemization reaction allows interconversion of the amine enantiomers and continuously feeds the enzyme catalyzed reaction with more reactive enantiomer (Fig. 1.24 B and C). The hybrid catalyst proved to be exceptionally active for the DKR of 1-phenylethylamine and ethyl methoxy acetate with quantitative yield and ee of $99 \%$. Besides these representative examples, many other articles and reviews have been published dealing with silica-based multi-functional catalysts featuring various combinations of active sites. ${ }^{17,97-102}$

Layered structures also provide interesting synthetic alternatives for preparing multifunctional catalysts. Corma et al. prepared a bi-functional acid-base hybrid catalyst starting from laminar MWW zeolite (Fig. 1.25). For that, firstly, they proceeded with swelling of the layered zeolitic precursors, in presence of cetyltrimethylammonium hydroxide (CTMA). The positively charged alkylammonium groups in the surfactant molecules facilitated the interaction with the negative charges of the zeolitic framework. Secondly, 
the swollen material was suspended in a dioxane solution of 1,4-bis(triethoxysilyl) benzene (BTEB). This aryl-bridged silsesquioxane molecules were able to diffuse in the interlayer space between the zeolites layers and to covalently bind them through the alkoxide groups. The swelling agents were removed by acid extraction. X-ray diffraction allowed to observe the presence of dimeric pillaring moieties constituted by two condensed BTEB molecules located in the interlayer space. Lastly, the aromatic bridging moieties were functionalized with amine groups, to obtain the bi-functional catalyst. Specifically, the pillared hybrid MWW-BTEB material was reacted in a $\mathrm{HNO}_{3} / \mathrm{H}_{2} \mathrm{SO}_{4}$ mixture to incorporate $-\mathrm{NO}_{2}$ groups, which were then reduced to $-\mathrm{NH}_{2}$ through reaction with $\mathrm{SnCl}_{2}$. The final material featured a hierarchical system of pores and the presence of the two active sites in different pore frameworks. Specifically, the acid sites were located within the micropores of the zeolitic layers and the basic amino groups were located in the mesopores formed by intercalation of the organic pillaring agents.
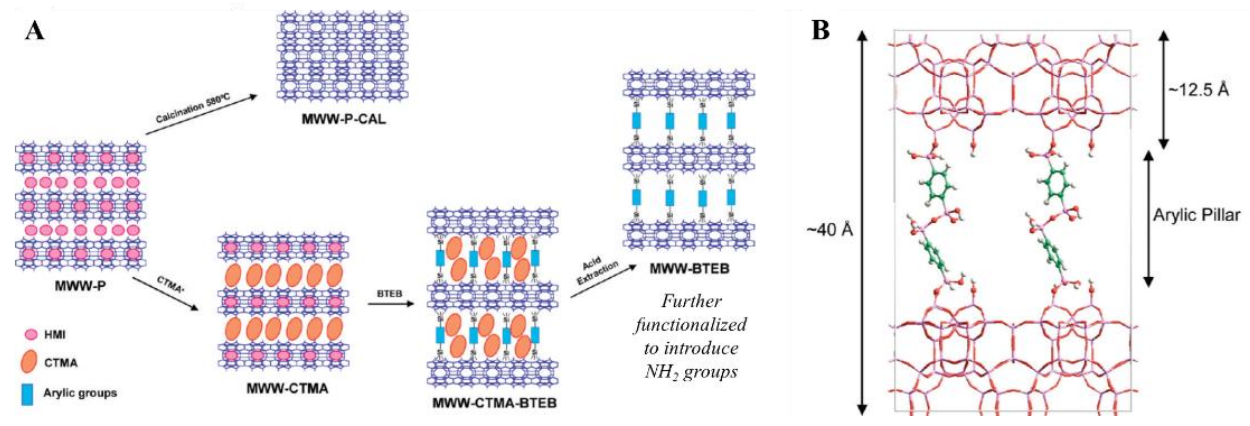

Figure 1.25 A: Synthetic procedure implemented for the synthesis of the pillared hybrid zeolitic materials from MWW precursors. B: Layered hybrid material obtained by pillaring with BTEB silsesquioxane molecules (MWW-BTEB). ${ }^{103}$

The catalyst was tested in a cascade reaction, involving an acetal hydrolysis followed by a Knoevenagel condensation. Specifically, benzaldehyde dimethylacetal was hydrolyzed to produce benzaldehyde, which was reacted with malononitrile to give benzylidene malononitrile. The results showed high activity of the catalyst in the tandem reaction, with a product yield of $96 \%$ after $7.2 \mathrm{~h}$ of reaction and a selectivity of $99 \% .{ }^{103}$

In another study, Gaona et al. prepared functional acid-base hybrid materials based on (organo)aluminosilicate layers prepared through direct hydrothermal synthesis processes in the presence of aluminum-rich alkaline media and two combined types of organosiloxane precursors. They were able to achieve isolated aluminosilicate sheets with amino and/or sulfonic groups homogeneously distributed along their external surface, located 
between the layers. The $\mathrm{NH}_{2}$-functionalized hybrid material was prepared by hydrothermal synthesis in which triethoxymethylsilane (TEMS) and 3-aminopropyl trimethoxysilane (APTMS) were implemented as silicon precursors together with sodium aluminate $\left(\mathrm{NaAlO}_{2}\right)$ and $\mathrm{NaOH}$. The slurry was stirred at room temperature until gelation occurred and it was then transferred into a stainless steel autoclave and heated at $135^{\circ} \mathrm{C}$ for 9 days under autogenous pressure and static conditions. Finally, the solid was washed with distilled water and dried. A catalyst featuring $\mathrm{HSO}_{3}$ moieties was prepared, as well, by using as silicon precursors a mixture of TEMS and 3-mercaptopropyl trimethoxysilane (MPTMS). The thiol groups resulting from the use of the MPTMS were oxidized to sulfonic acids by stirring the material in an acid ethanolic solution $\left(0.02 \mathrm{M} \mathrm{H}_{2} \mathrm{SO}_{4}\right)$ at room temperature for $24 \mathrm{~h}$. Finally, by using TEMS, APTMS and MPTMS as silicon sources, the authors were able to prepare laminar materials featuring both $\mathrm{NH}_{2}$ and $\mathrm{HSO}_{3}$ groups. Individual nanolayers were able to interact with each other through multiple types of interactions, such as Van der Waals, hydrogen or ionic interactions, allowing to obtain layered crystalline materials without using a structure directing agent. Different catalytic reactions were implemented to test the activity of the different hybrids. The condensation of carbonyl compounds with active methylenic substrates, in reactions such as Knoevenagel and Henry was used to probe the activity of $\mathrm{NH}_{2}$-functionalized catalyst, whereas acetalization of benzaldehyde with methanol to 1,1-dimethoxytoluene was used to test the activity of the $\mathrm{HSO}_{3}$-functionalized material. The performances of the bi-functional acid-base catalysts were evaluated in one-pot two-step process involving an acid-catalyzed acetal hydrolysis followed by a base-catalyzed Knoevenagel reaction, with a product yield of $92 \%$. This study provided a valid alternative to the typical mesoporous silica for applications as inorganic supports for the synthesis of multi-functional hybrid organic-inorganic catalysts. ${ }^{104}$ 
Chapter 1

\subsection{References}

(1) Chorkendorff, I.; Niemantsverdriet, J. W. Concepts of Modern Catalysis and Kinetics , 2nd ed.; 2007, Wiley-VCH.

(2) Gallego, E. M.; Paris, C.; Cantín, Á.; Moliner, M.; Corma, A. Chem. Sci. 2019, 80098015 .

(3) Gallego, E. M.; Portilla, M. T.; Paris, C.; León-Escamilla, A.; Boronat, M.; Moliner, M.; Corma, A. Science 2017, 355, 1051-1054.

(4) Wohlfahrt, G.; Witt, S.; Hendle, J.; Schomburg, D.; Kalisz, H. M.; Hecht, H. J. 1999, 55 (5), 969-977.

(5) Donoeva, B.; de Jong, K. P. Nat. Mater. 2020, 19, 5-6.

(6) Kamat, P. V. J. Phys. Chem. B 2002, 106, 7729-7744.

(7) Jain, P. K.; Huang, X.; El-Sayed, I. H.; El-Sayed, M. A. Plasmonics 2007, 2, 107 118.

(8) Liu, L.; Corma, A. Chem. Rev. 2018, 118, 4981-5079.

(9) Qiao, B.; Wang, A.; Yang, X.; Allard, L. F.; Jiang, Z.; Cui, Y.; Liu, J.; Li, J.; Zhang, T. Nat. Chem. 2011, 3, 634-641.

(10) Wang, A.; Li, J.; Zhang, T. Nat. Rev. Chem. 2018, 2, 65-81.

(11) Sanchez, C.; Julián, B.; Belleville, P.; Popall, M. J. Mater. Chem., 2005, 15, 35593592.

(12) Martins Estevão, B.; Miletto, I.; Marchese, L.; Gianotti, E. Phys. Chem. Chem. Phys. 2016, 18, 9042-9052.

(13) Parejo, P. G.; Alvarez-Herrero, A.; Zayat, M.; Levy, D. J. Mater. Sci. 2015, 50, 6677-6687.

(14) Toki, M.; Chow, T. Y.; Ohnaka, T.; Samura, H.; Saegusa, T. Polym. Bull. 1992, 29, 653-660.

(15) Saegusa T; Chujo Y. Polym Prep 1989, 1, 39.

(16) Sanchez, C.; Ribot, F. New J. Chem. 1994, 18, 1007-1047.

(17) Díaz, U.; Brunel, D.; Corma, A. Chem. Soc. Rev. 2013, 42, 4083-4097.

(18) Ghiaci, M.; Abbaspur, A.; Kia, R.; Seyedeyn-Azad, F. Sep. Purif. Technol. 2004, 40, 217-229.

(19) Yang, K.; Chen, B.; Zhu, L. Sci. Rep. 2015, 5, 1-12.

(20) Gaona, A.; Moreno, J. M.; Velty, A.; Díaz, U.; Corma, A. J. Mater. Chem. A 2014, 2, 19360-19375. 
(21) Jose Maria Moreno Rodriguez, Sintesis de Materiales Hybridos Metal-Organicos Estructurados a Partir de Unidades Unidimensionales. Estudio de Sus Propriedades y Aplicaciones, 2018, Doctoral Thesis, Universitat Politecnica de Valencia.

(22) Kalmutzki, M. J.; Hanikel, N.; Yaghi, O. M. Sci. Adv. 2018, 4.

(23) Morris, R. E. J. Mater. Chem. 2005, 15, 931-938.

(24) Tranchemontagne, D. J.; Mendoza-Cortés, J. L.; O’Keeffe, M.; Yaghi, O. M. Chem. Soc. Rev. 2009, 38, 1257-1283.

(25) Hailian, L.; Eddaoudi M.; O'Keeffe M.; Yaghi O. M. Nature 1999, 402, 276-279

(26) Surblé, S.; Serre, C.; Mellot-Draznieks, C.; Millange, F.; Férey, G. Chem. Commun. 2006, 3, 284-286.

(27) O’Keeffe, M.; Yaghi, O. M. Chem. Rev. 2012, 112, 675-702.

(28) Rowsell, J. L. C.; Yaghi, O. M. Microporous Mesoporous Mater. 2004, 73, 3-14.

(29) Konstas, K.; Osl, T.; Yang, Y.; Batten, M.; Burke, N.; Hill, A. J.; Hill, M. R. J. Mater. Chem. 2012, 22, 16698-16708.

(30) Kaye, S. S.; Dailly, A.; Yaghi, O. M.; Long, J. R. J. Am. Chem. Soc. 2007, 129, 14176-14177.

(31) D'vries, R. F.; Camps, I.; Ellena, J. Cryst. Growth Des. 2015, 15, 3015-3023.

(32) Hou, K. L.; Bai, F. Y.; Xing, Y. H.; Wang, J. L.; Shi, Z. Inorganica Chim. Acta 2011, 365, 269-276.

(33) Li, H.; Wang, K.; Sun, Y.; Lollar, C. T.; Li, J.; Zhou, H. C. Mater. Today 2018, 21, 108-121.

(34) García-García, P.; Müller, M.; Corma, A. Chem. Sci. 2014, 5, 2979-3007.

(35) Xin, R.; Yu, X. Y.; Gao, W. P.; Wang, N.; Yang, J. J.; Qu, X. S.; Zhang, X. Inorg. Chem. Commun. 2013, 35, 38-41.

(36) Zhao, X.; Liu, S.; Tang, Z.; Niu, H.; Cai, Y.; Meng, W.; Wu, F.; Giesy, J. P. Sci. Rep. 2015, 5, 1-10.

(37) Wu, M. X.; Yang, Y. W. Adv. Mater. 2017, 29, 1-20.

(38) Farha, O. K.; Eryazici, I.; Jeong, N. C.; Hauser, B. G.; Wilmer, C. E.; Sarjeant, A. A.; Snurr, R. Q.; Nguyen, S. T.; Yazaydin, A. Ö.; Hupp, J. T. J. Am. Chem. Soc. 2012, 134, 15016-15021.

(39) Trickett, C. A.; Helal, A.; Al-Maythalony, B. A.; Yamani, Z. H.; Cordova, K. E.; Yaghi, O. M. Nat. Rev. Mater. 2017, 2, 1-16.

(40) Chen, B.; Ockwig, N. W.; Millward, A. R.; Contreras, D. S.; Yaghi, O. M. Angew. Chemie - Int. Ed. 2005, 44, 4745-4749. 
(41) Peng, Y.; Krungleviciute, V.; Eryazici, I.; Hupp, J. T.; Farha, O. K.; Yildirim, T. J. Am. Chem. Soc. 2013, 135, 11887-11894.

(42) Ma, S.; Wang, X. Sen; Yuan, D.; Zhou, H. C. Angew. Chemie - Int. Ed. 2008, 47, 4130-4133.

(43) Cadiau, A.; Adil, K.; Bhatt, P. M.; Belmabkhout, Y.; Eddaoudi, M. Science 2016, $353,137-140$.

(44) Chughtai, A. H.; Ahmad, N.; Younus, H. A.; Laypkov, A.; Verpoort, F. Chem. Soc. Rev. 2015, 44, 6804-6849.

(45) Alaerts, L.; Séguin, E.; Poelman, H.; Thibault-Starzyk, F.; Jacobs, P. A.; De Vos, D. E. Chem. - A Eur. J. 2006, 12, 7353-7363.

(46) Wu, F.; Qiu, L. G.; Ke, F.; Jiang, X. Inorg. Chem. Commun. 2013, 32, 5-8.

(47) Kleist, W.; Jutz, F.; Maciejewski, M.; Baiker, A. Eur. J. Inorg. Chem. 2009, 24, 3552-3561.

(48) Dhakshinamoorthy, A.; Opanasenko, M.; Čejka, J.; Garcia, H. Catal. Sci. Technol. 2013, 3, 2509-2540.

(49) Hoffmann, F.; Cornelius, M.; Morell, J.; Fröba, M. Angew. Chemie - Int. Ed. 2006, 45, 3216-3251.

(50) Asefa, T.; MacLachlan, M. J.; Coombs, N.; Ozin, G. A. Nature 1999, 402, 867-871.

(51) Inagaki, S.; Guan, S.; Fukushima, Y.; Ohsuna, T.; Terasaki, O. J. Am. Chem. Soc. 1999, 121, 9611-9614.

(52) Fujita, S.; Inagaki, S. Chem. Mater. 2008, 20, 891-908.

(53) Melde, B. J.; Holland, B. T.; Blanford, C. F.; Stein, A. Chem. Mater. 1999, 11, 3302 3308 .

(54) Inagaki, S.; Guan, S.; Ohsuna, T.; Terasaki, O. Nature 2002, 416, 304-307.

(55) Mizoshita, N.; Tani, T.; Inagaki, S. Chem. Soc. Rev. 2011, 40, 789-800.

(56) Iler, R. K. Chem. Silica Solubility, Polym. Colloid Surf. Prop. Biochem. Silica 1979, $172-311$.

(57) Jeffrey Brinker, C. Colloid. Silica Fundam. Appl. 2005, 615-635.

(58) Osterholtz, F. D.; Pohl, E. R. J. Adhes. Sci. Technol. 1992, 6, 127-149.

(59) Schmidt, H.; Scholze, H.; Kaiser, A. J. Non. Cryst. Solids 1984, 63, 1-11.

(60) Bernards, T. N. M.; van Bommel, M. J.; Boonstra, A. H. J. Non. Cryst. Solids 1991, 134, 1-13.

(61) Winter, R.; Chan, J. B.; Frattini, R.; Jonas, J. J. Non. Cryst. Solids 1988, 105, 214 222. 
(62) Pope, E. J. A.; Mackenzie, J. D. J. Non. Cryst. Solids 1986, 87, 185-198.

(63) Reale, E.; Leyva, A.; Corma, A.; Martínez, C.; García, H.; Rey, F. J. Mater. Chem. 2005, 15, 1742-1754.

(64) Yilmaz, B.; Müller, U. Top. Catal. 2009, 52, 888-895.

(65) Kaur, M.; Sharma, S.; Bedi, P. M. S. Chinese J. Catal. 2015, 36, 520-549.

(66) Kourasi, M.; Wills, R. G. A.; Shah, A. A.; Walsh, F. C. Electrochim. Acta 2014, 127, 454-466.

(67) Tsukuda, E.; Sato, S.; Takahashi, R.; Sodesawa, T. Catal. Commun. 2007, 8, 13491353.

(68) Vázquez, P.; Pizzio, L.; Cáceres, C.; Blanco, M.; Thomas, H.; Alesso, E.; Finkielsztein, L.; Lantaño, B.; Moltrasio, G.; Aguirre, J. J. Mol. Catal. A Chem. 2000, $161,223-232$.

(69) Arias, M.; Laurenti, D.; Geantet, C.; Vrinat, M.; Hideyuki, I.; Yoshimura, Y. Catal. Today 2008, 130, 190-194.

(70) Aoyama, T.; Takido, T.; Kodomari, M. Synlett 2004, 2, 2307-2310.

(71) Maleki, B.; Baghayeri, M. RSC Adv. 2015, 5, 79746-79758.

(72) Khatik, G. L.; Sharma, G.; Kumar, R.; Chakraborti, A. K. Tetrahedron 2007, 63, 1200-1210.

(73) Bandgar, B. P.; Patil, A. V.; Chavan, O. S. J. Mol. Catal. A Chem. 2006, 256, 99105.

(74) Rajput, V. K.; Roy, B.; Mukhopadhyay, B. Tetrahedron Lett. 2006, 47, 6987-6991.

(75) Doustkhah, E.; Luque, R.; Kim, J.; Lin, J.; Ide, Y.; Yamauchi, Y.; Rostamnia, S.; Wu, K. C.-W.; Len, C.; Luo, X.; Bando, Y. Chem. - A Eur. J. 2018, 25, 1614-1635.

(76) Cauvel, A.; Renard, G.; Brunel, D. J. Org. Chem. 1997, 62, 749-751.

(77) Wang, Q.; Shantz, D. F. J. Catal. 2010, 271, 170-177.

(78) Shimizu, K.; Suzuki, H.; Hayashi, E.; Kodama, T.; Tsuchiya, Y. Chem. Commun. 2002, 2, 16-17.

(79) Bass, J. D.; Solovyov, A.; Pascall, A. J.; Katz, A. J. Am. Chem. Soc. 2006, 128, 37373747.

(80) Balbino, J. M.; De Menezes, E. W.; Benvenutti, E. V.; Cataluña, R.; Ebeling, G.; Dupont, J. Green Chem. 2011, 13, 3111-3116.

(81) Barbarini, A.; Maggi, R.; Mazzacani, A.; Mori, G.; Sartori, G.; Sartorio, R. Tetrahedron Lett. 2003, 44, 2931-2934.

(82) Motos-Pérez, B.; Roeser, J.; Thomas, A.; Hesemann, P. Appl. Organomet. Chem. 
2013, 27, 290-299.

(83) Gianotti, E.; Diaz, U.; Coluccia, S.; Corma, A. Phys. Chem. Chem. Phys. 2011, 13, 11702-11709.

(84) Li, P.; Wang, L.; Zhang, Y.; Wang, G. Tetrahedron 2008, 64, 7633-7638.

(85) Llopis, S.; García, T.; Cantín, Á.; Velty, A.; Díaz, U.; Corma, A. Catal. Sci. Technol. 2018, 8, 5835-5847.

(86) Zamboulis, A.; Moitra, N.; Moreau, J. J. E.; Cattoën, X.; Wong Chi Man, M. J. Mater. Chem. 2010, 20, 9322-9338.

(87) Zhang, Q.; Su, H.; Luo, J.; Wei, Y. Tetrahedron 2013, 69, 447-454.

(88) Del Pozo, C.; Corma, A.; Iglesias, M.; Sánchez, F. Green Chem. 2011, 13, 2471 2481.

(89) Baleizão, C.; Gigante, B.; Das, D.; Álvaro, M.; Garcia, H.; Corma, A. J. Catal. 2004, 223, 106-113.

(90) Budroni, G.; Corma, A. Angew. Chemie - Int. Ed. 2006, 45, 3328-3331.

(91) Baleizão, C.; Gigante, B.; Das, D.; Alvaro, M.; Garcia, H.; Corma, A. Chem. Commun. 2003, 3, 1860-1861.

(92) Jiang, Y.; Gao, Q. J. Am. Chem. Soc. 2006, 128, 716-717.

(93) Budroni, G.; Corma, A.; García, H.; Primo, A. J. Catal. 2007, 251, 345-353.

(94) Gianotti, E.; Diaz, U.; Velty, A.; Corma, A. Catal. Sci. Technol. 2013, 3, 2677-2688.

(95) Sharma, K. K.; Biradar, A. V.; Das, S.; Asefa, T. Eur. J. Inorg. Chem. 2011, 21, 3174-3182.

(96) Johnston, E. V.; Verho, O.; Kärkäs, M. D.; Shakeri, M.; Tai, C. W.; Palmgren, P.; Eriksson, K.; Oscarsson, S.; Bäckvall, J. E. Chem. - A Eur. J. 2012, 18, 12202-12206.

(97) Zeidan, R. K.; Hwang, S. J.; Davis, M. E.. Angew. Chemie - Int. Ed. 2006, 45, 63326335 .

(98) Huh, S.; Chen, H. T.; Wiench, J. W.; Pruski, M.; Lin, V. S. Y. Angew. Chemie - Int. Ed. 2005, 44, 1826-1830.

(99) Patankar, S. C.; Dodiya, S. K.; Yadav, G. D. J. Mol. Catal. A Chem. 2015, 409, 171182.

(100) Huang, Y.; Trewyn, B. G.; Chen, H. T.; Lin, V. S. Y. New J. Chem. 2008, 32, 1311 1313.

(101) Fernandes, A. E.; Jonas, A. M. Catal. Today 2019, 334, 173-186.

(102) Li, P.; Cao, C. Y.; Liu, H.; Yu, Y.; Song, W. G. J. Mater. Chem. A 2013, 1, 12804 12810. 
Introduction to Functional Hybrid Catalysts

(103) Corma, A.; Díaz, U.; García, T.; Sastre, G.; Velty, A. J. Am. Chem. Soc. 2010, 132, 15011-15021.

(104) Gaona, A.; Díaz, U.; Corma, A. Chem. Mater. 2017, 29, 1599-1612. 


Chapter 2

\section{General Objectives}



As it was highlighted in Chapter 1, the development of synthetic strategies for obtaining hybrid organic-inorganic heterogeneous catalysts featuring multiple active sites are becoming ever more attractive, due to the perspective of developing cascade processes that would represent greener and more environmentally-friendly alternatives for the fine chemicals and pharmaceutical industries. The incorporation of multiple catalytic sites within a single material is not a trivial task and usually require a custom-made synthetic approach for each new organic-inorganic hybrid. Many aspects need to be considered, such as surface and textural properties of the support, the choice of the organic fragments to incorporate into the solid, the impact of a simultaneous (versus sequential) incorporation of several catalytic sites, the interactions between pendant organic moieties and the surface of the support, the hydrophilicity/hydrophobicity of the solid's surface and its impact on the overall catalytic activity, amongst others.

Probably, the most reasonable approach to this complicated task is to first study the properties of a mono-functional catalyst and of the one-step catalytic process in which it is implemented, then proceeding with the incorporation of a second active site and with the development of a multi-step cascade process. This approach is the backbone of this Doctoral Thesis, which describes the development of different classes of hybrid catalysts that were designed upon the needs of the industrial partners of the MULTI2HYCAT European project. For all of the above, the general objectives are the following:

- Mono-functional hybrid organic-inorganic silica-based heterogeneous catalysts featuring new super-acid catalytic sites, specifically (fluoro)aryl-sulfonic acid groups, will be synthesized, following a multi-step synthesis process starting from commercially available substrates.

- The acid hybrid catalysts will be thoroughly characterized and the effect of the fluorinated aromatic ring on its catalytic activity will be studied in the acetal formation reaction between benzaldehyde and ethylene glycol. Moreover, a silanol-capped version of the hybrids will be prepared and the effect of the hydrophilicity of the surface will be studied.

- For the first time, organic probe molecules will be adsorbed onto acid organic-inorganic silica-based hybrid catalysts and will be studied by NMR and FTIR spectroscopies. Probe molecules will include carbon monoxide, ammonia and trimethylphosphine oxide.

- Silica based hybrid catalysts featuring base groups (amine or N-heterocyclic ammonium hydroxides) will be prepared using MCM-41 as support. The catalysts will be tested in three different catalytic reactions involving C-C bond formation, i.e. Knoevenagel condensations between benzaldehyde and substrates featuring different $\mathrm{pKa}$ and the Michael addition between ethyl 2-oxocyclopentane 2-carboxylate and methyl vinyl ketone. Moreover, the catalysts were tested in the aldol condensation between furfural and methyl isobutyl ketone, which will then constitute the first step of a tandem process. The screening of the activity of the hybrids will provide 
information on the most suitable mono-functional base catalyst, onto which a second functionality will be later incorporated.

- A new, versatile synthetic approach to support phosphazenium hydroxide moieties onto silica-based supports will be presented to expand the family of silica-based hybrid catalyst containing active base groups.

- A multi-functional hybrid catalyst featuring a base group and metal nanoparticles will be developed, based upon previous results. The catalyst will be characterized and tested in a cascade catalytic process of industrial interest, i.e. the aldol condensation between furfural and methyl isobutyl ketone followed by the hydrogenation of the aldol adduct. The influence of several variables on the catalytic activity will be explored, in the perspective of more thorough optimization of the process to be carried out in flow regime.

- The synthesis of two multi-functional silica-based catalysts featuring base groups and metal complexes will also be reported. The first one will feature pendant aminopropyl species and palladium-based bis-N-heterocyclic-carbene complex moieties. The second one will be prepared from a bi-functional molecular platform derived from BINAP, provided by a partner of the MULTI2HYCAT consortium. The bi-functional hybrid will feature primary amine groups and Pd-BINAP-based complexes. The synthetic processes for preparing the hybrid catalysts will be described. Preliminary characterization results as well as a proof-of-concept example of catalytic application will also be reported. Specifically, the activity of the catalysts will be demonstrated in a tandem reaction involving a Suzuki coupling between benzeneboronic acid and 4-bromobenzaldehyde, followed by aldol condensation of the product with acetone. 




\section{Chapter 3}

\section{Organosiliceous Hybrid Materials Functionalized with Aryl-Sulfonic Acid Groups}

Two different heterogeneous hybrid catalysts carrying aryl-sulfonic moieties, in which the aromatic ring was either fluorinated or not, were successfully synthesized. The multi-step synthetic approaches implemented involved the synthesis of a silyl-derivative precursor, template-free one-pot co-condensation (at low temperature and neutral $\mathrm{pH}$ ) and tethering reaction. A multitechnique approach was implemented to characterize the hybrid organic-inorganic catalysts. The catalytic activity of the two hybrids showed superior performances for the fluoro-aryl-sulfonic acid, compared to the non-fluorinated material.

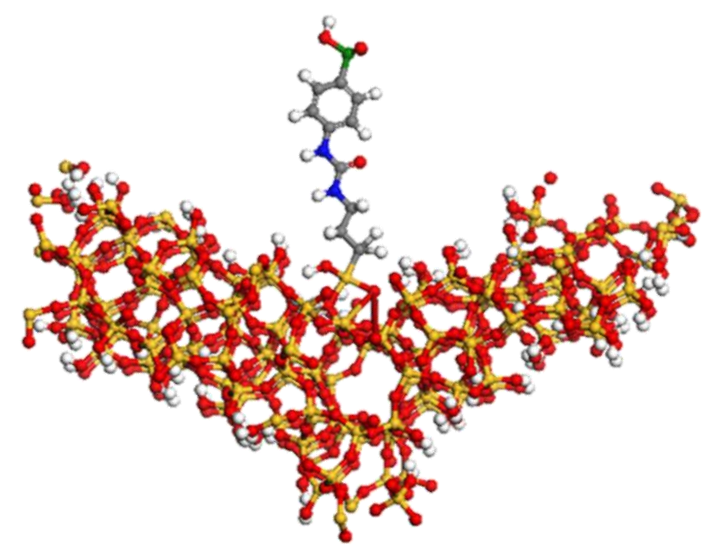





\subsection{Sulfonic acid groups in heterogeneous catalysis}

Sulfonic acids groups are one of the main organic functionalities implemented in the design of hybrid catalysts due to their strong Brönsted acidity and the ease with which they can be synthetized and incorporated into solid materials. The first reports of heterogeneous acid catalysis by resinous exchangers containing sulfonic acid groups date back to the late 1940s. ${ }^{1}$ In the following decades, sulfonic functionalized polymers became of great interest, specifically for catalytic applications in reactions such as ester hydrolysis ${ }^{2,3}$ alkylation of aromatics, ${ }^{4}$ synthesis of bisphenol A. ${ }^{5}$ Nowadays, sulfonated resins are implemented for a number of acid catalyzed reactions aimed to biodiesel production $^{6-12}$ and production of fine chemicals from biomass. ${ }^{13,14}$

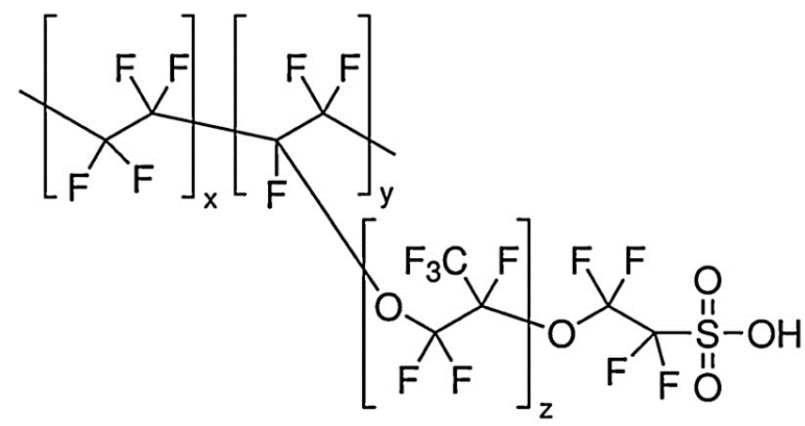

Figure 3.1: Schematic representation of the structure of Nafion polymers.

The most important examples of sulfonic acid catalysts are undoubtedly Nafion ${ }^{15}$ (Fig. 3.1) and Amberlist ${ }^{16}$ resins. Though these catalysts are more expensive than mineral acids, advantages such as lower corrosion and environmental effects related to the acid waste treatment and ease of product separation often compensate for their high costs. Additionally, when compared with other heterogeneous catalysts (e.g. zeolites, metal oxides), they present unique features that make them much more suitable for certain reactions. Firstly, the strong sulfonic acid group confers greater acidity; moreover, the surface properties of the polymer differ from those of inorganic oxides. This feature is beneficial, for example, in the production of biodiesel where the hydrophobicity of the polymer backbone promotes the interaction with the tails of alcohol and fatty acids which, in turn, facilitate enhanced catalytic activity. ${ }^{17}$ Due to the pore-swelling of the resins in organic reaction media, mass transfer limitations are usually minimized with consequently enhanced accessibility of reactant to the acid functionalities. ${ }^{18}$ Nevertheless, this behavior is strongly influenced by the reaction medium. ${ }^{19-22}$ For example, Suarez et al. reported a study on the use of $\mathrm{Sn}$ (3-hydroxy-2-methyl 4-pyrone $)_{2}\left(\mathrm{H}_{2} \mathrm{O}\right)_{2}$ as catalyst for methanolysis that lost its activity when it was immobilized onto ion exchange resin. This was ascribed to the poor swelling properties of the Purolite CT-275 in a polar 
medium such as methanol. On the other hand, resins that had higher degree of swelling due to increased divinylbenzene contents, displayed higher yields of methyl esters. ${ }^{23}$ Although undoubtedly promising for some reactions, resins present some drawbacks that limit their overall applicability as a support for heterogeneous catalysts. Firstly, due to their organic nature, resins present low thermal stability, which narrows the range of applicability to low-boiling mixtures. ${ }^{24}$ Secondly, their swelling behavior must be kept into account for their usage in fixed bed reactors. ${ }^{25}$

Another aspect to consider, more related to the scope of this Thesis, is that the choice of the support on which the active sites are incorporated is a major factor to keep into account while designing a multi-functional catalyst. Catalytically active sites are intrinsically reactive functional groups, which makes their incorporation and co-existence into a single material at the nanoscale not trivial. For all of the above, a class of supports more versatile than resins are required if one wants to incorporate sulfonic acid groups into the design of a multi-functional catalyst. Ideally, the support should be mechanically and thermally stable, its surface should be mildly reactive and its hydrophilic/hydrophobic properties should be tunable. Mesoporous silica supports are one of the primary candidates for such role. Thermal and mechanical stability, compared to that of the organic moieties they would be meant to support, and high surface area are just some of the advantages that they bring about. The highly reactive surface allows for multiple steps of functionalization, which can involve the addition of a second catalytic functionality, the change in the hydrophilic/hydrophobic nature of the surface, the deposition of metal nanoparticles and others.

Before deepening into the state of the art of the research on silica supported sulfonic acids and highlighting the objectives of the work reported in this Chapter, it would be useful to give a brief outlook on the other materials that are being used to support sulfonic acid groups.

\subsubsection{Sulfonic acids supported onto mesoporous carbons}

Mesoporous carbons have recently been developed. They are synthetized by hard- or soft-templating carbonization. Due to their high stability and potential for undergoing sulfonation, mesoporous carbons have attracted great interest for the development of supported sulfonic acid moieties for applications requiring high temperatures. ${ }^{26-28} \mathrm{Wu} e t$ al. pioneered a vapor-phase sulfonation of controllable carbonized CMK-3, in autoclave with pure $\mathrm{H}_{2} \mathrm{SO}_{4}$ followed by treatment with pure $\mathrm{SO}_{3}$ gas. ${ }^{28}$ They also studied the influence of carbonization on the functionalization of CMK-3 at temperatures ranging between 673 and $1173 \mathrm{~K}$, at which a highly ordered mesoporous carbon was obtained (Fig. 3.2 A). In the synthesis of these materials, the presence of polycyclic aromatic rings and their preservation throughout the carbonization process is a crucial parameter, as the sulfonation occurs on these sites. The catalyst was implemented in the Beckman rearrangement of cyclohexanone oxime and in the condensation of bulky aromatic aldehydes. 


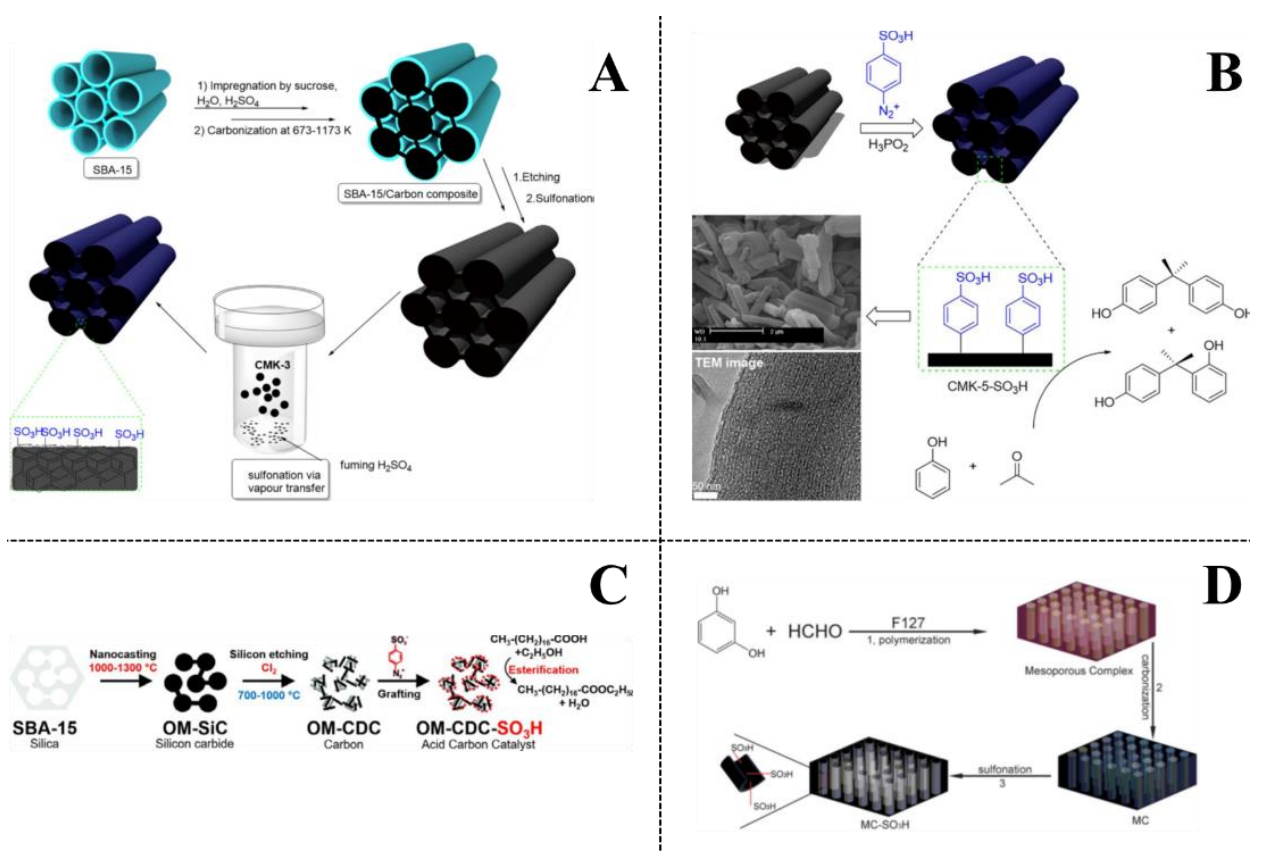

Figure 3.2 A: functionalization of $\mathrm{CMK}-3$ with fuming $\mathrm{H}_{2} \mathrm{SO}_{4}{ }^{28} \mathrm{~B}$ : sulfonation of CMK-5 with benzene para-diazonium sulfonic acid. ${ }^{29} \mathrm{C}$ : synthesis of $\mathrm{SiC}$-derived $\mathrm{SO}_{3} \mathrm{H}$-functionalized mesoporous carbon by hard-templating approach. ${ }^{26} \mathrm{D}$ : Synthesis of $\mathrm{SO}_{3} \mathrm{H}$-functionalized mesoporous carbon by soft-templating approach. ${ }^{27}$

An alternative approach implemented for the preparation of sulfonated CMK-5 involved the reduction of the diazonium salts, containing a sulfonic acid functional group, and hypophosphorous acid. ${ }^{29}$ The final material was tested as catalyst in the production of bisphenols (Fig. 3.2 B). Although, the catalyst resulted to be less active than $\mathrm{H}_{2} \mathrm{SO}_{4}$ the study provided a recoverable, and more reusable solid acid catalyst which offered a greener route for the production of bisphenoles. Li et al. anchored phenylsulfonic acid groups onto the surface of ordered mesoporous carbons by in situ radical polymerization of isoamyl nitrite and sulfanilic acid under ambient conditions. ${ }^{30}$ By using this approach, they reduced the number of synthetic steps to one. The hybrid was then used as support for palladium nanoparticles, achieved by deposition of $\mathrm{PdCl}_{2}$ and subsequent reduction with $\mathrm{NaBH}_{4}$. The catalyst was successfully employed in the electro-oxidation of formic acid. Corma $e t$ al. recently designed a new carbide functionalized with sulfonic acid groups, which was derived from ordered silicon carbide $(\mathrm{SiC})$. The resulting catalyst showed an extraordinarily high specific surface area (up to $2800 \mathrm{~m}^{2} / \mathrm{g}$ ). ${ }^{26}$ The strategy was based upon the selective extraction of silicon from ordered mesoporous $\mathrm{SiC}$, followed by functionalization with sulfonic groups. The hybrid material showed excellent catalytic properties in the esterification of stearic acid to ethyl stearate (Fig. 3.2 C). 
Another strategy recently developed as an alternative to nanocasting and hard templating methods involves the self-assembly of organic molecules and soft templating routes. As an example, Dong et al. prepared ordered mesoporous nanocarbons bearing sulfonic acid groups in a three-step process in which F127 was used as structural directing agent (SDA) (Fig. 3.2 D) ${ }^{27}$ The material obtained was carbonized at various temperatures. Carbonization at $400{ }^{\circ} \mathrm{C}$ was found to be optimal, providing a highly active catalyst towards the esterification of oleic acid with methanol.

\subsubsection{Sulfonated mesoporous polymers}

Organic mesoporous polymers can be designed to work as catalysts, bearing sulfonic acid groups as active sites. ${ }^{31}$ These materials are particularly advantageous due to the ease with which they can be synthesized and modified. Their porosity can be generated either through steric orientation of the building blocks, occurring during the polymerization process (template-free method) ${ }^{32}$ or by addition of a templating agent, during polymerization (templating method). ${ }^{33}$ In spite of their potential, there are not many reports of sulfonated organic mesoporous polymers in the scientific literature. Ryoo et al. implemented a free-radical polymerization approach starting from cross-linkable olefinic monomers and using mesoporous silica KIT- 6 as hard templating agent. ${ }^{34}$ After carrying out the polymerization reaction at $150{ }^{\circ} \mathrm{C}$, the sulfonation step was conducted using sulfuric acid to $90{ }^{\circ} \mathrm{C}$ (Fig. 3.3). The removal of the silica template was achieved by using hydrofluoric acid.

A

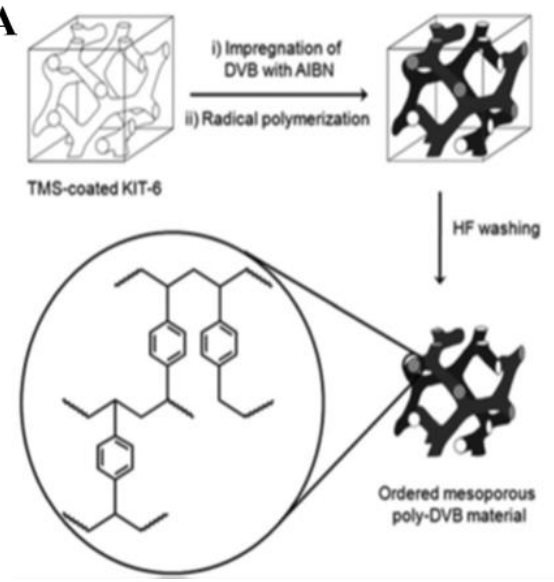

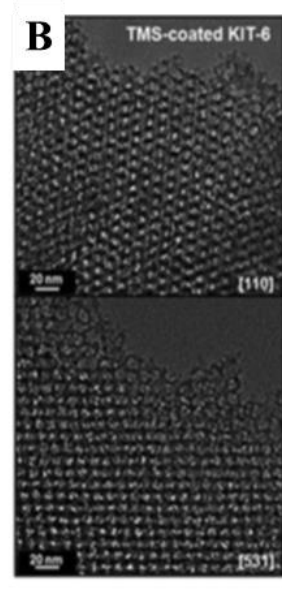

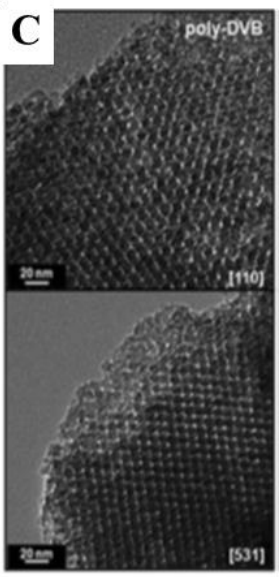

Figure 3.3 A: Synthesis of $\mathrm{SO}_{3} \mathrm{H}$-functionalized ordered mesoporous polymer through poly(divinylbenzene)(poly-DVB) by using trimethoxy silane (TMS)-coated KIT-6 as templating agent. TEM images of TMS-coated KIT-6 (B) and poly-DVB materials(C). AIBN=azobisisobutyronitrile. ${ }^{34}$ 
Under the optimized replication conditions, the material showed a very narrow pore size distribution and a high specific surface area, which is coherent with the textural properties of the mesoporous silica that was used as template. The material was tested as catalyst in the esterification of hexanoic acid with benzyl alcohol, with performances superior to those of commercially available Amberlist- $15 .{ }^{34}$

\subsubsection{Sulfonic acids supported onto silica-based materials}

Since 1998, sulfonic acid groups have been supported onto mesoporous silicas. ${ }^{35,36}$ In the first studies on these hybrid acid catalysts, two approaches were typically implemented: (i) post synthetic grafting of 3-mercaptopropyltrimethoxysilane (MPTMS) followed by oxidation of the thiol groups to sulfonic acid moieties and (ii) co-condensation of MPTMS with TEOS or TMOS, also followed by oxidation. Since their introduction, many advances have been made, such as increasing the MPTMS loading through a coating method, ${ }^{37}$ replacing calcination with extraction methods, ${ }^{38}$ and co-functionalization of MPTMS and octyl substituents to enhance the catalytic activity by increasing the hydrophobicity of the hybrid catalyst. ${ }^{39}$ Supported sulfonic acid catalysts are primarily employed in biomass conversion. ${ }^{37}$ However, their versatility allowed their successful implementation in other catalytic processes such as synthesis of 2,2-bis(5methylfuryl)propane by condensation of acetone with 2-methylfuran, ${ }^{35}$ esterification of d-sorbitol with lauric acid, ${ }^{40}$ synthesis of monolaurin by direct esterification of glycerol with lauric acid, ${ }^{41}$ Fries and Beckmann rearrangements, ${ }^{42,43}$ syntheses of polyhydroquinoline derivatives, ${ }^{44}$ synthesis of $\beta$-amino carbonyls through Mannich reactions, ${ }^{45}$ and others. ${ }^{37}$

\subsubsection{Precursors for sulfonation of silica matrixes}

Several sulfonic acid precursors have been reported for the sulfonation of mesoporousmaterials. From the point of view of the inclusion of sulfonic acid groups into the final hybrid materials, different approaches can be followed: (i) implementation of commercial sulfur-containing organosiloxanes and eventual conversion of the sulfur-based functional groups to sulfonic acid moieties, usually carried out after incorporation into the solid material (e.g. MPTMS); (ii) synthesis of new silyl-derivatives containing sulfonic acid groups; (iii) functionalization of supported organic moieties with sulfuric acid ${ }^{37,46,47}$. In Fig. 3.4, some organic moieties that have been successfully used to synthesize silicasupported sulfonic acid catalysts are reported. Unsurprisingly, using different siloxane precursors influences the properties of the final catalyst, across the board. A few examples are reported below. 


\begin{tabular}{|c|c|c|}
\hline Entry & Structural formula & Properties and applications ${ }^{[a]}$ \\
\hline 1 & & -a famous family of SAPs generated through the oxidation of MPTMS \\
\hline 2 & & $\begin{array}{l}\text {-prepared in one step prior to functionalization and attached to a silica } \\
\text { surface through the siloxane moiety; } \\
\text {-strongly acidic and highly hydrophobic; } \\
\text { expensive to produce }\end{array}$ \\
\hline 3 & & $\begin{array}{l}\text {-can be directly functionalized onto a silica surface; } \\
\text {-strongly acidic due to fluorine atoms; } \\
\text {-higher leaching and more expensive }\end{array}$ \\
\hline 4 & & $\begin{array}{l}\text {-attaches to a silica surface through the siloxane moiety; } \\
\text {-by adding a nucleophile, a wide variety of bifunctionalized SAMs } \\
\text { can be produced }\end{array}$ \\
\hline 5 & & $\begin{array}{l}\text {-can directly attach to a silica surface or undergo ring opening } \\
\text { in the presence of an amine }\end{array}$ \\
\hline 6 & & $\begin{array}{l}\text {-can be prepared in two steps: } \\
\text { 1) functionalization of PTS onto a silica surface; } \\
\text { 2) sulfonation of a phenyl group by } \mathrm{H}_{2} \mathrm{SO}_{4} \text {; } \\
\text {-their acidity is similar to that of commercial sulfonated Amberlyst-15 resins }\end{array}$ \\
\hline 7 & & $\begin{array}{l}\text {-can be easily hydrolyzed to give other } \mathrm{SAPs} \text { and can be used to tailor } \\
\text { surfaces through nucleophile attack of }-\mathrm{SO}_{2} \mathrm{Cl} \text { moieties; } \\
\text {-these kinds of precursors can decrease the pore size and surface area }\end{array}$ \\
\hline 8 & & $\begin{array}{l}\text {-similar to the precursor in entry } 6 \text {, except with more flexibility and a longer length; } \\
\text {-these two SALs are more water tolerant than that of the SAL shown in entry } 1\end{array}$ \\
\hline 9 & $n=2,4$ & $\begin{array}{l}\text {-can be embedded into a silica wall and then oxidized to }-\mathrm{SO}_{3} \mathrm{H} \text { to give } \\
\text { a uniform distribution of sulfonyl groups }\end{array}$ \\
\hline 10 & $\begin{array}{l}\mathrm{RO} \\
\mathrm{RO} \\
\mathrm{RO}\end{array}$ & $\begin{array}{l}\text {-can be prepared from chlorosulfonic acid and APTS; } \\
\text {-converts into a zwitterion in water and slowly hydrolyzes to the amine and } \mathrm{H}_{2} \mathrm{SO}_{4}\end{array}$ \\
\hline 11 & & $\begin{array}{l}\text {-generally used for MCs and functionalized through displacement of the } \\
\text { diazonium }{ }^{[34]} \text { in the presence of } \mathrm{H}_{3} \mathrm{PO}_{2}\end{array}$ \\
\hline
\end{tabular}

Figure 3.4: List of some of the precursors implemented in the synthesis of silica supported sulfonic acids. SAP: sulfonic acid precursors; SAMs: sulfonic acid based mesostructures; PTS: phenyltrialkoxysilane; SALs: sulfonic acid ligands; MCs: mesoporous carbons. Adapted from ref. 37.

An increase in hydrophobicity and consequent improvement in catalyst deactivation by water and mass transfer of hydrophobic compounds was achieved by using phenylsulfonic acid derivatives instead of propylsulfonic acid moieties. ${ }^{37}$ Phenylsulfonic acid siloxane-based precursors have been used by several authors to synthesize supported sulfonic acid materials that have shown superior catalytic properties when compared both with others non-commerial/non-aromatic catalysts and with commercial resins such as Amberlyst-15 (Fig. 3.4, entries 6, 7, 8, 11). ${ }^{26,48-51}$ Lindlar et al. reported the synthesis of MCM-41-Ph-SO $3 \mathrm{H}$ (Fig. 3.4, entry 6) ${ }^{37}$ with pore diameters of up to $6 \mathrm{~nm}$ by implementing swelling agents. Firstly, the phenyl groups were grafted onto the silica surface; subsequently, chlorosulfonic acid was used for sulfonation. However, this latter acid could also react with free silanols. To avoid this phenomenon, capping agents, such 
as trimethoxymethylsilane, were grafted after functionalization with the phenyl precursor. For the sulfonation of mesoporous silica materials, grafting is the most widely used method, due to the advantages over co-condensation method, the main one being SDA removal by calcination rather than by extraction, which implies the use of solvent and it is usually not completely effective. Nevertheless, Gedeona et al. found that after extraction, further calcination with slow heating ramp up to $200{ }^{\circ} \mathrm{C}$ could completely remove P123 from the extracted sample. ${ }^{52}$

The functionalization of fluorinated sulfonic acid precursors inside the mesopores was first reported by Harmer et al. in 1997. ${ }^{53}$ These hybrid mesoporous materials are strongly acidic due to the presence of electronegative fluorine atoms. However, the preparation and stability of these hybrids are major drawbacks as they would often undergo leaching and consequent deactivation. ${ }^{48}$ 1,2,2-Trifluoro-2-hydroxy-1-trifluoromethylethane sulfonic acid sultones (Fig. 3.4, entry 3) could be directly anchored onto silica surfaces through a direct synthetic strategy. ${ }^{54}$ The same authors reported another strategy for the preparation of fluorinated supported sulfonic acids through a platinum-catalyzed hydrosilylation procedure. The resulting silyl-derivative could then be anchored onto the silica surface through a co-condensation reaction or grafting. ${ }^{55}$

Recently, supported N-propylsulfamic acid groups have drawn significant attention in the field. Sulfamic acid based catalysts can be regarded as strong acids that are zwitterionic in the absence of water (Fig. 3.4; entry 10). ${ }^{56,57}$ These catalysts are easily separable from reaction mixtures and can be recycled different times if supported on the surface of mesopores. Under alkaline or neutral $\mathrm{pH}$, sulfamic acid derivatives can be boiled without appreciable hydrolysis. However, they slowly hydrolyze under acid conditions, giving a supported primary amine and releasing a molecule of sulfuric acid. ${ }^{58}$

From this overview, it appears clear that supporting complex sulfonic-functionalized moieties is not a trivial task, especially when green chemistry standards need to be met. Phenyl-sulfonic acid catalysts often require using either corrosive agents for sulfonation of aromatic precursors or expensive pre-sulfonated reactants, to begin with. Even less trivial is the synthesis of fluorinated compounds, with the few silyl-derivatives reported being synthesized through low-yielding steps, starting from expensive reactants. There is room for improvement also in the synthetic strategies implemented to obtain the silicasupported hybrid catalysts. The use of expensive surfactants and of acid/base reactants in the sol-gel processes, as well as the high calcination temperatures, are also aspects that need to be kept into account when designing a hybrid catalyst with potential scaling-up perspectives.

\subsubsection{Sulfonated periodic mesoporous organosilicas (PMOs)}

As mentioned before, many studies have been carried out with the scope of tuning the hydrophilic/hydrophobic nature of supported sulfonic acid materials, due to the influence that this parameter has on their catalytic activity. This is particularly important, because one of the main catalytic applications of supported sulfonic acid moieties involves the 
use of hydrophobic fatty acids as reactants..$^{59,60}$ Silanols-capping with hydrophobic groups and implementation of hydrophobic sulfonated silyl-derivatives are viable alternatives, which nevertheless present disadvantages such as decreasing the pore size and consequent mass transfer limitations in the case of bulky compounds. ${ }^{61}$ Periodic mesoporous organosilicas (PMOs) are often regarded as the next-generation of these catalysts, with sufficient hydrophobicity and crystal-like pore walls. The first sulfonated benzenebridged PMO (Ph-PMO- $\left.\mathrm{SO}_{3} \mathrm{H}\right)$ was synthesized through the condensation of 1,4-bis(triethoxysilyl)benzene (BTEB) in presence of specific SDAs. The white solid obtained was then sulfonated with a $25 \%$ solution of $\mathrm{SO}_{3} / \mathrm{H}_{2} \mathrm{SO}_{4}$. The same authors also carried out a co-condensation of BTEB and MPTMS under basic conditions, in the presence of a SDA, achieving a Ph-PMO with pendant thiol groups, subsequently converted to sulfonic acid by treatment with $\mathrm{HNO}_{3}$ at room temperature for $24 \mathrm{~h}^{62}$

The use of acidic SBA-15-like PMOs, in which the location of the acid sites could be controlled has been proposed and tested in acid-catalyzed reactions in hot water (Fig. 3.5). ${ }^{63}$ The hybrids were prepared either by the condensation of BTEB, followed by sulfonation using chlorosulfonic acid (catalyst 1 ) or by MPTMS condensation and further oxidation with $\mathrm{H}_{2} \mathrm{O}_{2}$ (catalyst 2).

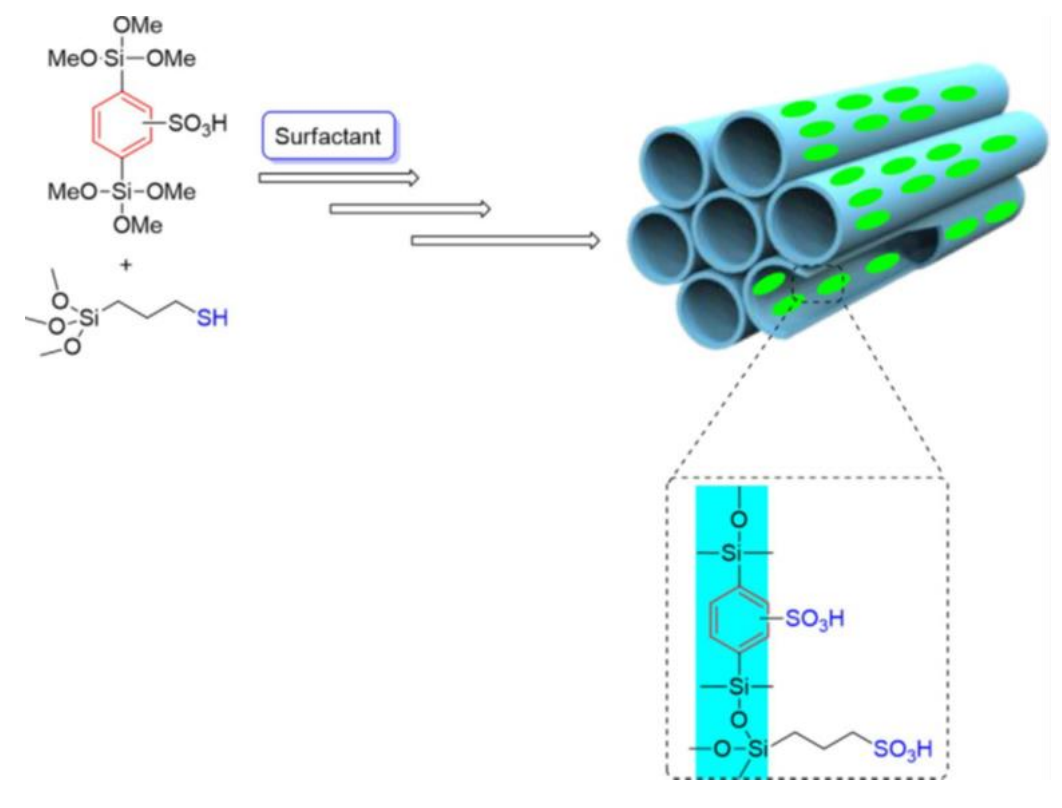

Figure 3.5: Synthesis of double-sulfonated phenylene-bridged $\mathrm{PMO}_{-} \mathrm{SO}_{3} \mathrm{H}^{63}$ 
The acidity of the materials was studied by calorimetric measurements of the adsorption of ammonia. The catalytic activity of the materials was tested in the gas-phase dehydration of isopropanol, used as a model reaction, and compared with the reference acid sulfonated resin, i.e. Amberlyst- 15 .

While the oxidation step of MPTMS thiol groups to sulfonic acid groups in $\mathrm{H}_{2} \mathrm{O}_{2}$ did not affect the mesoscopic structure, the treatment with chlorosulfonic acid, to incorporate sulfonic acid moieties in the structural aromatic units, did affect it. In spite of the partially compromised structure, catalyst 1 (obtained by treatment with chlorosulfonic acid) showed catalytic activity in the gas-phase dehydration of isopropanol equivalent to that of Amberlyst-15, although with progressive partial loss of the activity due to the leaching of sulfur species. On the other hand, SBA-15-like PMOs obtained by $\mathrm{H}_{2} \mathrm{O}_{2}$ oxidation of the thiol groups (catalyst 2) showed lower catalytic activity in the gas-phase dehydration of isopropanol. However, catalyst 2 did not show any significant leaching of sulfur species was observed.

The catalytic performances of these materials were also evaluated in biomass valorization, such as cellobiose hydrolysis in hot water. At $150{ }^{\circ} \mathrm{C}$ the catalysts showed significant leaching of sulfur species with the catalytic activity observed mainly due to species performing in homogeneous phase. Extensive washing of the catalysts prior to the treatment somehow improved their stability and recyclability. The washing step probably acted by removing the labile species that leached in the catalytic tests. Consequently and unsurprisingly, this led to lower catalytic activity in the first cycle. ${ }^{63}$

In another example, Jerome et al. also studied different types of silica supported sulfonic acid materials and monitored the catalytic activities for the aqueous synthesis of bis(indolyl)methanes. ${ }^{64}$ They extensively studied the effect of hydrophobicity on the catalytic activities and found that an increase in hydrophobicity led to better catalytic performances for this reaction. By comparing Ph-PMO-Pr- $\mathrm{SO}_{3} \mathrm{H}$ and SBA-15- $\mathrm{PrSO}_{3}$ catalysts, the former, in which phenyl groups are present within the pore walls, showed higher catalytic activity (Fig. 3.6) ${ }^{65}$ The versatility of silica-based synthesis, allows for the incorporation of multiple functionality either to improve the catalytic efficiency of a specific active site or to carry out multi-step cascade reactions. As an example of the former, in some catalytic processes, the activity of sulfonic acid catalysts benefits from the presence of amine-type functionalities. 


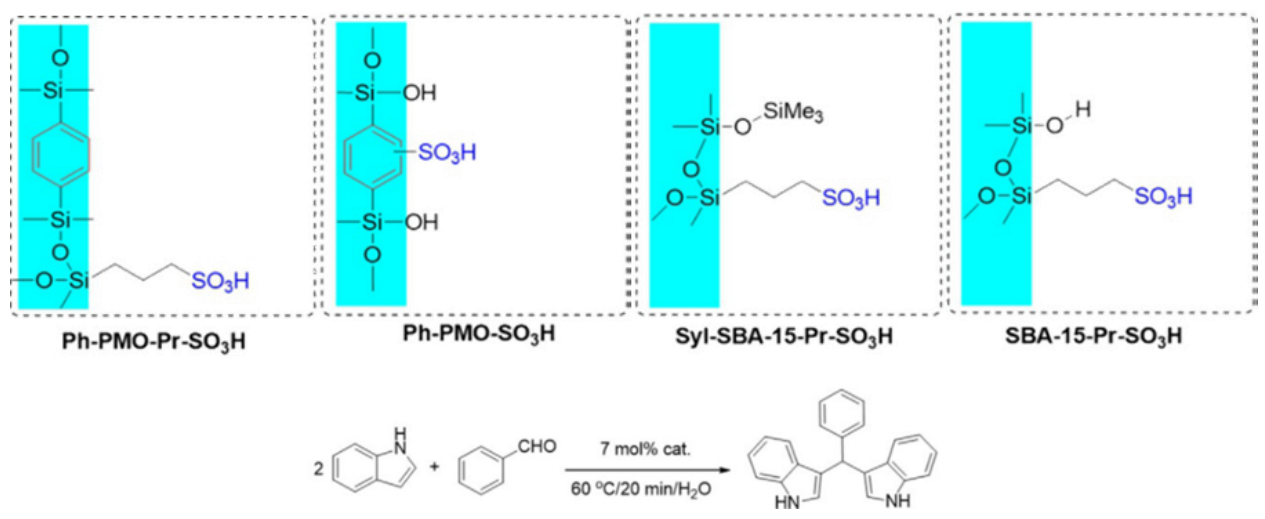

Figure 3.6: Comparing the catalytic activities of four different types of sulfonated mesoporous materials. Adapted from ref. 65.

Thiel et al. introduced amine groups via post-synthesis functionalization of a BTEBbased $\mathrm{PMO}-\mathrm{SO}_{3} \mathrm{H}$ obtaining a bi-functional PMO (Fig. 3.7). ${ }^{66}$ Firstly, BTEB was reacted with tert-butyloxycarbonyl(Boc)-protected APTS in the presence of SDA molecules. The resulting PMO structure was then sulfonated with chlorosulfonic acid. Finally, the protecting group was removed from the catalyst. The catalyst was tested in nitroaldol condensation reactions, with excellent yields. The authors also tested the catalytic activity of $\mathrm{N}$-protected $\mathrm{PMO}-\mathrm{SO}_{3} \mathrm{H} /-\mathrm{NHBoc}$ in nitroaldol condensation reaction, recording no conversion. Interestingly, the protected system exhibited remarkable activity in the conversion of dimethoxyphenyl-methane to benzaldehyde. This catalyst was also compared with monofunctionalized mesoporous materials $\left(\mathrm{SBA}-\mathrm{NH}_{2}\right.$ and $\left.\mathrm{PMO}-\mathrm{SO}_{3} \mathrm{H}\right)$ with both catalysts performing worse than the bi-functional catalyst $\mathrm{PMO}-\mathrm{SO}_{3} \mathrm{H} /-\mathrm{NH}_{2}$.

In another relevant study Alauzun et al. reported the synthesis a bi-functional mesoporous material containing two antagonist functions, i.e. an acidic group in the framework and a basic one in the channel pores. The material was synthesized by co-condensation of a mixture of a bridged disilane featuring a disulfur group - $(\mathrm{MeO})_{3} \mathrm{Si}-\left(\mathrm{CH}_{2}\right)_{3}-\mathrm{S}-\mathrm{S}$ $\left(\mathrm{CH}_{2}\right)_{3}-\mathrm{Si}(\mathrm{OMe})_{3}(\mathrm{~S} 2)$; tetraethyl orthosilicate (TEOS); a silyl-derivative featuring a protected amine group - $(\mathrm{MeO})_{3} \mathrm{Si}-(\mathrm{CH} 2)_{3}-\mathrm{NHBoc}$. The synthesis was carried out in the presence of non-ionic surfactant P123. The final bi-functional hybrid catalyst was obtained by post-synthetic reduction of disulfide units followed by their oxidation to $\mathrm{SO}_{3} \mathrm{H}$ groups and deprotection of amino groups. ${ }^{67}$ 


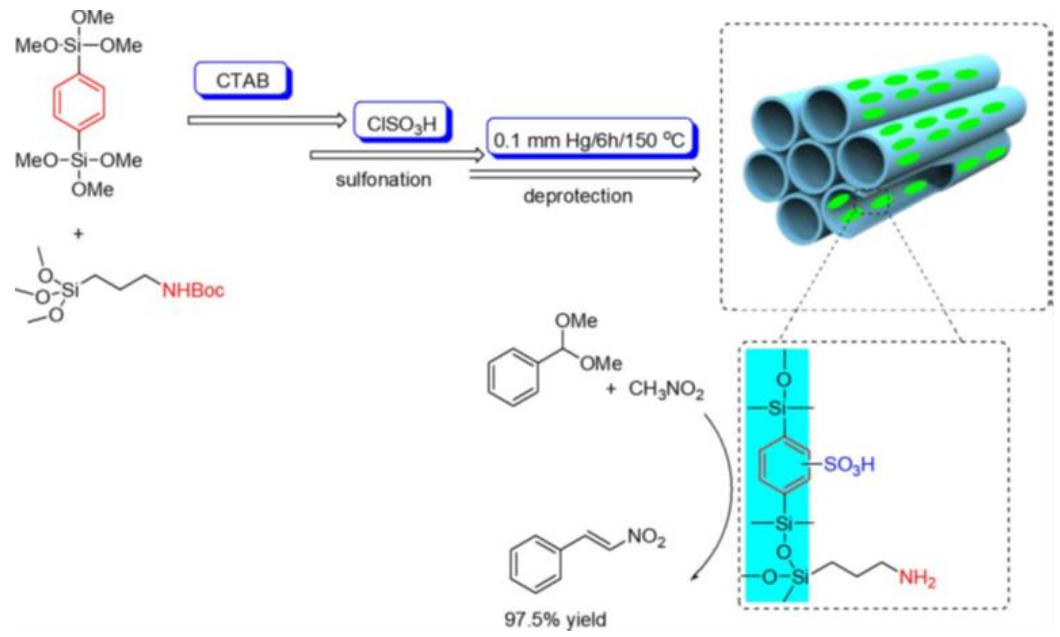

Figure 3.7: Synthesis of a bi-functionalized PMO for nitroaldol catalytic reactions. ${ }^{66}$ 


\subsection{Scope of this work}

As mentioned before, the research work reported in this Thesis was carried out as part of the broader scope of the MULTI2HYCAT project. Being the project ultimately focused on the development of multi-functional catalysts, the general strategy implemented for the design of the supported sulfonic acids kept into account the eventual further use of the building blocks here described (carrying the acid sites) for the synthesis of catalysts baring multiple catalytic sites.

The ease with which silica can be functionalized, tuning the hydrophilic/hydrophobic character of the surface and/or incorporating multiple catalytic functionalities ${ }^{46,68}$ make silica-based strategies the most appealing ones, to this scope. Among the many synthetic procedures available for incorporating organic moieties into silica materials, fluoridecatalyzed co-condensation of a silyl-derivative, baring the functional group of interest, and tetramethyl ortosilicate (TMOS) is one of the less explored, yet more advantageous, especially from a "green" perspective. This is due to the low temperature, neutral $\mathrm{pH}$, absence of templating agent and low quantity of solvent implemented. The resulting nonordered mesoporous silicas (NOS) are typically characterized by high specific surface area, relatively narrow pore size distribution and reactive surface.

Focusing on the design of the organic part of the hybrid, the effect of nearby atoms on the catalytic activity of sulfonic acids has been studied for several decades. For example, propyl- and aryl- sulfonic acids have been supported on amorphous silicas and their activity was compared in the reaction of anisol with acetic anhydride ${ }^{69} \mathrm{~A}$ beneficial effect of having the sulfonic group bound to an aromatic ring was observed. This effect can be ascribed to the electron-donating effect of the aromatic ring, compared to a completely $\mathrm{sp}^{3}$ hybridized alkyl chain. Moreover, fluorinated alkylsulfonic acids have been exploited by the chemical industry for decades, with the above mentioned Nafion and Amberlist resins, being the best example. In fact, the inductive effect provided by having fluorine as a substituent on the carbon in the $\alpha$ position greatly enhances the acidity of the proton. This clearly highlights the effect that the chemical makeup of the sulfonic acid moieties can have on the catalytic activity. ${ }^{70-73}$ Nevertheless, the synergistic effect of having the sulfonic group bounded to a fluorinated aromatic ring has not been explored.

Based on the considerations reported above, in this Chapter the main objectives will be the following:

- The synthesis of two supported aryl-sulfonic acid organocatalysts based on NOStype materials. The final aim will be to study the inductive effect of fluorine on the aryl-sulfonic groups and, ultimately, on their acidity. One of the hybrids will feature a fluorinated aromatic ring, while the other will feature an aromatic ring unsubstituted in positions $2,3,5$ and 6 . 
- The two hybrid materials will undergo in-depth physico-chemical characterization aimed at studying textural properties, morphology, spectroscopic properties and thermal stability. Specifically, a combination of FTIR and multi-nuclear solid state NMR methods will be implemented.

- Probe molecules of different basic strengths will be adsorbed/desorbed onto the hybrids. Gaseous or highly volatile bases such as carbon monoxide, ammonia, pyridine and pyridine derivatives had been extensively used as probe molecules to study the acidic properties of purely inorganic catalysts. ${ }^{74-76}$ However, within the field of organosiliceous hybrid catalysts, this insightful characterization approach is still unexplored. In the work reported in this Chapter, recently published ${ }^{77}$ for the first time, carbon monoxide, ammonia and trimethylphosphine oxide will be adsorbed on the surface of the hybrids and their interaction will be followed both by FTIR and solid state NMR spectroscopies in order to assess the acidity of the hybrid catalysts.

- A passivated version of the catalysts, in which the surface silanols are reacted with hexamethyl disilazane, will be prepared.

- The catalytic activity of the two hydrophobic hybrids will be tested in acetal formation reactions and compared with that of the non-passivated ones, in order to access the influence of the surface silanols on the overall performances of the materials. 


\subsection{Experimental}

\subsubsection{Synthesis of the materials}

Compounds are labelled according to scheme reported in Fig. 3.8.

Synthesis of silyl-derivative 2. Sulfanilic acid (99\%, Aldrich) was reacted with 1 equivalent of $\mathrm{NaOH}$, in $\mathrm{H}_{2} \mathrm{O}$, to give its sodium form. The salt was reacted with 3-(triethoxysilyl)propyl isocyanate (95\%, Aldrich) in acetonitrile, overnight, at $65{ }^{\circ} \mathrm{C}$, under $\mathrm{N}_{2}$. The solvent was then evaporated under vacuum.

Synthesis of hybrid 3. A solution of silyl-derivative 2, tetramethyl orthosilicate (98\%, Aldrich), $\mathrm{H}_{2} \mathrm{O}$ and $\mathrm{MeOH}$ was prepared and stirred until complete homogeneity of the slurry was reached (solution $\mathrm{A}$ ). A solution of $\mathrm{NH}_{4} \mathrm{~F}$ (Aldrich, $98 \%$ ) in $\mathrm{H}_{2} \mathrm{O}$ was prepared (solution B). Solution B was added to solution A and stirred at room temperature until gelation occurred. The gel obtained was aged for $24 \mathrm{~h}$ at $36{ }^{\circ} \mathrm{C}$. After that, the solid was dried overnight at $65{ }^{\circ} \mathrm{C}$, ground and washed with $\mathrm{H}_{2} \mathrm{O}$ and ethanol. The final molar composition of the synthesis gel was as follows: $0.97 \mathrm{TMOS}: 0.03 \mathrm{RSi}\left(\mathrm{OR}^{\prime}\right)_{3}: 4 \mathrm{MeOH}$ : $4 \mathrm{H}_{2} \mathrm{O}: 0.00313 \mathrm{NH}_{4} \mathrm{~F}$. (Silyl-derivative 2 indicated as $\mathrm{RSi}\left(\mathrm{OR}^{\prime}\right)_{3}$ in the molar composition).

Synthesis of hybrid 4 . The material was obtained using the same procedure as the synthesis of 3, using (3-bromopropyl)- trimethoxysilane (97\%, Fluorochem) instead of silylderivative 2 .

Synthesis of 6. A solution of 5 (sodium 2,3,5,6-tetrafluoro-4-hydroxybenzenesulfonate, $98 \%$, TCI Europe) in DMSO was prepared under $\mathrm{N}_{2}$. DBU (1,5-diazabiciclo(5.4.0)undec-7-ene, 97.5\%, Aldrich) was added and the resulting mixture was stirred for 10 minutes. Br-Propyl functionalized silica (hybrid 4) was added and the mixture was stirred for $16 \mathrm{~h}$ at $100{ }^{\circ} \mathrm{C}$. Afterwards the suspension was cooled down at room temperature and the solid was filtered and washed abundantly with $\mathrm{H}_{2} \mathrm{O}$ and ethanol.

Synthesis of $\mathrm{HSO}_{3}-\mathrm{NOS}-\mathrm{H}$ and $\mathrm{HSO}_{3}-\mathrm{NOS}-\mathrm{F}$. The silica-supported sodium sulfonates 3 and 6 were washed three times with a $0.05 \mathrm{M} \mathrm{HCl}$ solution in $\mathrm{EtOH}$ at room temperature to achieve the protonic form.

Synthesis of $\mathrm{HSO}_{3}-\mathrm{CH}_{3}$ NOS-H: Hybrid 3 was placed in a Schlenck and outgassed at 150 ${ }^{\circ} \mathrm{C}$ for $2 \mathrm{~h}$. A solution of hexamethyldisilazane (HMDS) in DMSO was prepared under $\mathrm{N}_{2}$. After hybrid 3 cooled down, HMDS solution in DMSO was added to the Schlenck, under $\mathrm{N}_{2}$. The final molar composition of the mixture was $\mathrm{SiO}_{2}$ : 0.15HMDS : 10DMSO. The mixture was stirred at $120^{\circ} \mathrm{C}$ for $2 \mathrm{~h}$. The material was then filtered, washed with $\mathrm{CH}_{2} \mathrm{Cl}_{2}$ and dried overnight at $100^{\circ} \mathrm{C}$. The resulting hybrid was washed three times with a $0.05 \mathrm{M} \mathrm{HCl}$ solution in $\mathrm{EtOH}$ at room temperature to achieve the protonic form.

Synthesis of $\mathrm{HSO}_{3}-\mathrm{CH}_{3} \mathrm{NOS}-\mathrm{F}$ : Hybrid 4 was place in a Schlenck and outgassed at 150 ${ }^{\circ} \mathrm{C}$ for $2 \mathrm{~h}$. A solution of hexamethyldisilazane (HMDS) in DMSO was prepared under $\mathrm{N}_{2}$. After hybrid 4 cooled down, HMDS solution in DMSO was added to the Schlenck, 
under $\mathrm{N}_{2}$. The final molar composition of the mixture was $\mathrm{SiO}_{2}$ : 0.15HMDS : 10DMSO. The mixture was stirred at $120^{\circ} \mathrm{C}$ for $2 \mathrm{~h}$. The material was then filtered, washed with $\mathrm{CH}_{2} \mathrm{Cl}_{2}$ and dried overnight at $100{ }^{\circ} \mathrm{C}$. A solution of 5 in DMSO was prepared under $\mathrm{N}_{2}$. DBU was added and the mixture was stirred for 10 minutes. The passivated hybrid was added and the mixture was stirred at $100{ }^{\circ} \mathrm{C}$ for $16 \mathrm{~h}$. Afterwards the suspension was cooled down at room temperature and the solid was filtered and washed abundantly with DMSO and ethanol. Finally, the material was washed three times with a $0.05 \mathrm{M} \mathrm{HCl}$ solution in $\mathrm{EtOH}$ at room temperature to achieve the protonic form.

A

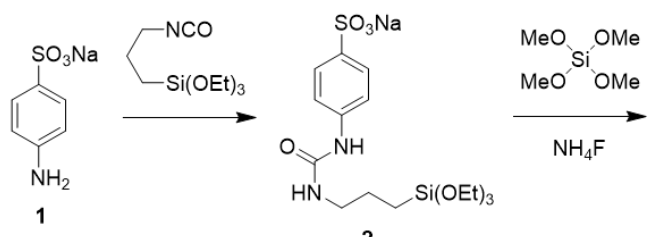

2

B
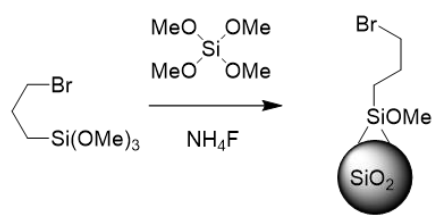

4

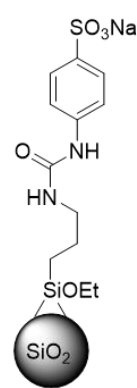

3
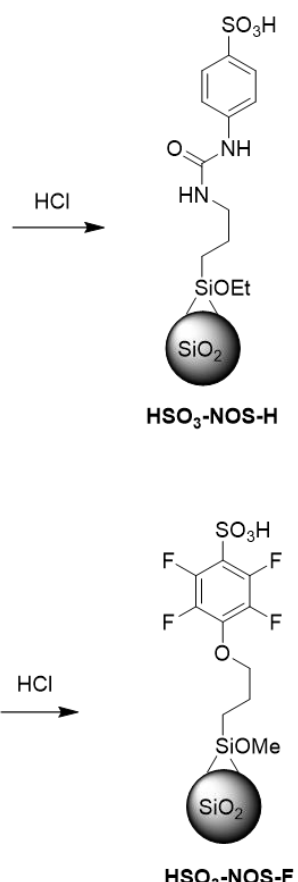

Figure 3.8: Schematic representation of the synthesis of the silica-supported sulfonic acids $\mathrm{HSO}_{3}$-NOS-H (A) and $\mathrm{HSO}_{3}$-NOS-F (B).

\subsubsection{Catalytic test}

For each catalytic run, the hybrid catalyst was added into a glass-lined reactor, equipped with a Dean Stark apparatus and a condenser. Aldehyde (1 eq., $0.5 \mathrm{mmol}$ ), dialcohol (30 
equiv.) and toluene $(5 \mathrm{ml})$ were added. The mixture was stirred at $120{ }^{\circ} \mathrm{C}$ for $20 \mathrm{~h}$. The yield was quantified by ${ }^{1} \mathrm{H}-\mathrm{NMR}$ using an internal standard.

Recycling: the crude reaction was filtered, and the catalyst was washed with dichloromethane, dried under vacuum and reused in the following reaction. The amount of catalysts used for each catalytic run was calculated based upon the quantity of S detected by elemental analysis, so that every reaction was catalyzed by the same amount of active sites.

\subsubsection{Characterization}

${ }^{1} \mathrm{H}$ and ${ }^{13} \mathrm{C}$ NMR spectra were recorded on a Bruker 300 spectrometer. The chemical shifts were reported in ppm, relative to residual proton solvent signals. Nitrogen adsorption isotherms were measured at $77 \mathrm{~K}$ with a Micromeritics ASAP 2010 volumetric adsorption analyzer. Prior to the measurement, the sample was outgassed for 12 hours at $80{ }^{\circ} \mathrm{C}$. The BET specific surface area was calculated from the nitrogen adsorption data in the relative pressure range from 0.04 to 0.2 . The total pore volume was obtained from the amount of $\mathrm{N}_{2}$ adsorbed at a relative pressure of about 0.99 . The pore size distribution and the pore diameter were obtained using the Barrett-Joyner-Halenda (BJH) method on the adsorption branch of the isotherms. The adsorption branch of the $\mathrm{N}_{2}$ physisorption isotherm was analyzed by means of the NLDFT (non-local density functional theory) method, to achieve the pore size distribution of the materials. Thermogravimetric analyses (TGA/DTA) of materials were performed under argon flow $\left(100 \mathrm{~mL} \mathrm{~min}^{-1}\right)$ with a SETSYS Evolution TGA-DTA/DSC thermobalance. The heating ramp was set from 40 to $1000{ }^{\circ} \mathrm{C}$ at $5{ }^{\circ} \mathrm{C} \mathrm{min}^{-1}$. The FTIR spectra of self-supporting pellets were collected under vacuum conditions (residual pressure $<10^{-5}$ mbar) using a Bruker Equinox $55 \mathrm{spec}$ trometer equipped with a pyroelectric detector (DTGS type) with a resolution of $4 \mathrm{~cm}^{-1}$. $\mathrm{CO}$ was adsorbed at $77 \mathrm{~K}$ and $\mathrm{NH}_{3}$ was adsorbed at room temperature using custom designed cells connected to a vacuum line to perform adsorption-desorption in situ measurements. The FTIR spectra were normalized upon the pellet weight and, whenever specified, are reported in difference-mode, i.e. by subtracting the spectrum of the sample under vacuum from the spectrum of the adsorbed molecules. Solid-state NMR spectra were acquired on a Bruker Advance III 500 spectrometer and a wide bore 11.7 Tesla magnet with operational frequencies for ${ }^{1} \mathrm{H},{ }^{13} \mathrm{C},{ }^{31} \mathrm{P}$ and ${ }^{29} \mathrm{Si}$ of $500.13,125.77,202.47$ and $99.35 \mathrm{MHz}$, respectively. A $4 \mathrm{~mm}$ triple resonance probe with MAS was employed for all the experiments. The samples were packed on a zirconia rotor and spun at a MAS rate between 10 and $15 \mathrm{kHz}$. The magnitude of radio frequency (RF) fields was 100 and $42 \mathrm{kHz}$ for ${ }^{1} \mathrm{H}$ and ${ }^{29} \mathrm{Si}$, respectively. The relaxation delay between accumulations was 2 and $2.5 \mathrm{~s}$. For ${ }^{13} \mathrm{C}$ and ${ }^{29} \mathrm{Si}$ cross polarization (CP) MAS experiments, RF fields of 55 and $28 \mathrm{kHz}$ were used for initial proton excitation and decoupling, respectively. During the $\mathrm{CP}$ period the ${ }^{1} \mathrm{H}$ RF field was ramped using 100 increments, whereas the ${ }^{13} \mathrm{C} /{ }^{29} \mathrm{Si}$ $\mathrm{RF}$ fields were maintained at a constant level. During the acquisition, the protons were decoupled from the carbon atoms/silicon atoms by using a two-pulse phase-modulated (TPPM) decoupling scheme. A moderate ramped RF field of $62 \mathrm{kHz}$ was used for spin 
locking, while the carbon/silicon RF field was matched to obtain the optimal signal. A rotor-synchronized spin-echo sequence $(\pi / 2-\tau-\pi-\tau$-acquisition) was also applied to record the ${ }^{1} \mathrm{H}$ NMR spectra with a delay time $\tau$ of $2 \mathrm{~ms}$. The delay time was chosen as an optimized compromise between the signal decay owing to relaxation and the resolution gain owing to longer delay times. ${ }^{31} \mathrm{P}$ MAS NMR spectra were recorded using high power proton decoupling and the RF field for the 90 -degree pulse was $83 \mathrm{kHz}$. All chemical shifts are reported using the $\delta$ scale and are externally referenced to tetramethylsilane (TMS) at $0 \mathrm{ppm}$ for ${ }^{1} \mathrm{H},{ }^{13} \mathrm{C}$ and ${ }^{29} \mathrm{Si}$. Ammonium dihydrogen phosphate (1 ppm) was used as a standard for the ${ }^{31} \mathrm{P}$ nucleus. Prior to the adsorption of TMPO probe molecules, the catalysts were packed onto a $4 \mathrm{~mm}$ zirconia rotor and outgassed at $180{ }^{\circ} \mathrm{C}$ for 90 minutes. After that, TMPO dissolved in anhydrous dichloromethane was added to the rotor and rapidly closed with a cap and left for adsorption for one hour. Later, the rotor was opened and attached back to the high vacuum line for the complete removal of dichloromethane at room temperature. Subsequently, the rotor was closed with a zirconia cap and heated to $160{ }^{\circ} \mathrm{C}$ for 30 minutes followed by submission to NMR spectral recording. 


\subsection{Results and discussion}

\subsubsection{Synthesis of the hybrid catalysts}

To vary the acid strength of the sulfonic hybrid catalysts, two different organic moieties, containing protonated or fluorinated aryl-sulfonic acid groups were designed. The syntheses of the two hybrid catalysts containing aryl-sulfonic groups were carried out following two different approaches showed in Fig. 3.9.

In the case of the hybrid materials with a protonated aromatic ring, named $\mathrm{HSO}_{3}$-NOS$\mathrm{H}$ (A), sulfanilic acid was firstly reacted with $\mathrm{NaOH}$ to achieve its sodium form (1) and improve its solubility in acetonitrile. The silyl derivative carrying the sodium sulfonate group (2) was obtained through the reaction of 3-(triethoxysilyl)propyl isocyanate with the primary amine present on the phenyl ring of 1 (acetonitrile, $65^{\circ} \mathrm{C}, \mathrm{N}_{2}$ ). The formation of the urea group was followed by liquid $\left({ }^{1} \mathrm{H}\right.$ and $\left.{ }^{13} \mathrm{C}\right)$ NMR spectra (Fig. 3.9). The peaks observed were consistent with the desired product. Specifically, the peak from ${ }^{13} \mathrm{C}$ NMR spectrum centered at $160 \mathrm{ppm}$ is typical of the carbonyl carbon present in the urea group. The synthesis of the solid catalyst was achieved by co-condensation of compound 2 and tetramethyl orthosilicate (TMOS), carried out in methanol and water (1:1). Hydrolysis and condensation of the alkoxy groups were catalyzed by $\mathrm{F}^{-}$ions from $\mathrm{NH}_{4} \mathrm{~F}$, which acted as mineralizing agents. ${ }^{78}$ This approach presents several advantages because the synthesis was carried out at $36^{\circ} \mathrm{C}$, for $24 \mathrm{~h}$, under mild $\mathrm{pH}$ conditions, using low amounts of solvent and catalyst and without using any structure directing agent. Additionally, the textural properties (specific surface area and mean pore size) can be tuned by varying the concentration of $\mathrm{F}^{-}$and the chain length of the alcohol used as a co-solvent. ${ }^{78}$

For the synthesis of the hybrid material with fluorinated aromatic ring, named $\mathrm{HSO}_{3-}$ NOS-F (B), a slightly different approach was used (Fig. 3.8). Compound 5 was found to be insoluble in any solvent besides $\mathrm{H}_{2} \mathrm{O}$ and DMSO. The implementation of water would have been detrimental to the alkoxy groups of the silyl-derivative precursor. On the other hand, DMSO would have required a very high temperature for its distillation. Such a high temperature might have promoted the spontaneous condensation of the alkoxy groups of the organosilane compounds and their partial polymerization. Given the impossibility of synthesizing the silyl-derivative alone and then grafting it, a $\mathrm{Br}$-propyl functionalized NOS was first prepared. Afterwards, the solid was reacted with compound 5 , previously mixed with DBU (1,5-diazabiciclo(5.4.0)undec-7-ene) to promote the deprotonation of the hydroxyl group. This last tethering step led to a non-porous hybrid material with pendant (fluoro)aryl-sulfonic groups (6). In both synthetic pathways, the exchange of $\mathrm{Na}^{+}$with $\mathrm{H}^{+}$was achieved by suspending hybrid 3 and 6 in a $0.5 \mathrm{M} \mathrm{HCl}$ solution in EtOH. The procedure was repeated three times. The total exchange of sodium was monitored by ICP analysis. 
A
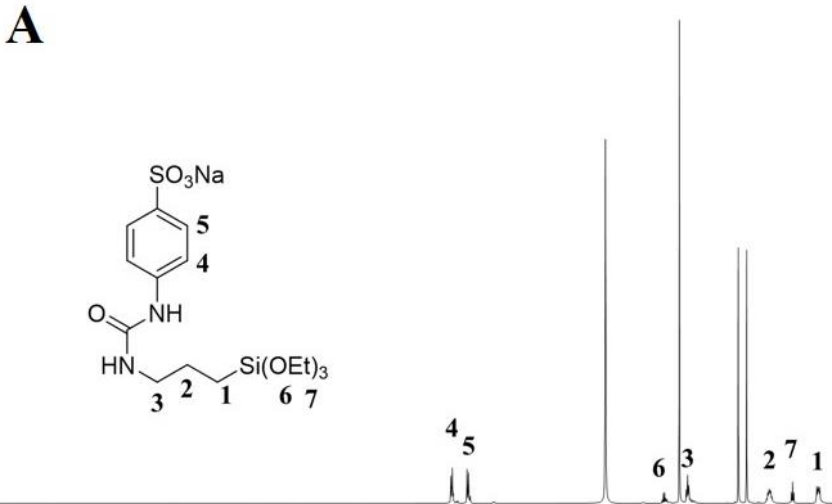

16

B

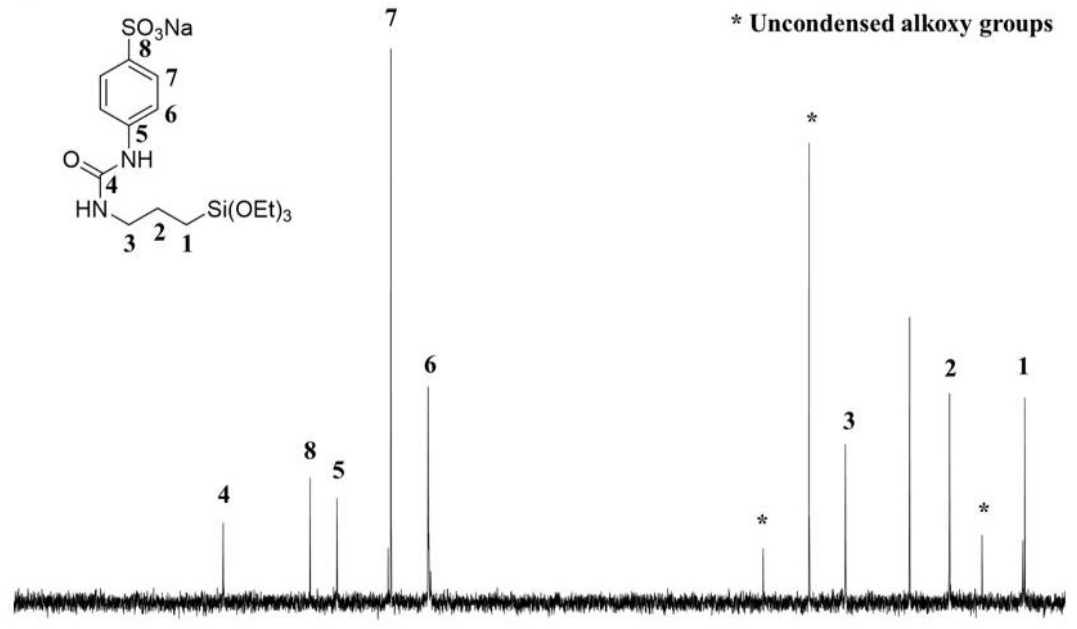

$\begin{array}{lllllllllllllllllll}190 & 180 & 170 & 160 & 150 & 140 & 130 & 120 & 110 & \begin{array}{c}100 \\ \mathrm{ppm}\end{array} & 90 & 80 & 70 & 60 & 50 & 40 & 30 & 20 & 10\end{array}$

Figure 3.9: ${ }^{1} \mathrm{H}(\mathrm{A})$ and ${ }^{13} \mathrm{C}$ (B) liquid NMR spectra of compound 2. 


\subsubsection{Characterization}

$\mathrm{HSO}_{3}$-NOS-H and $\mathrm{HSO}_{3}-\mathrm{NOS}-\mathrm{F}$ underwent in-depth physico-chemical characterization. Firstly, thermogravimetric analysis was carried out on both samples to probe the thermal stability of the materials and to measure the organic content. The weight losses (TGA) and the respective DTA curves of the materials are reported in Fig. 3.10. For both materials, the first weight loss occurs between 70 and $150{ }^{\circ} \mathrm{C}$ and it is associated with the removal of physisorbed water (I). Focusing on $\mathrm{HSO}_{3}-\mathrm{NOS}-\mathrm{H}$ (curves a and $\mathrm{a}^{\prime}$ ), a second weight loss is observed in the range between 250 and $380{ }^{\circ} \mathrm{C}$ (II). This is ascribed to the breakage of the urea bond and consequent desorption of the phenyl-sulfonic moieties. ${ }^{46}$ Lastly, the loss between 500 and $600{ }^{\circ} \mathrm{C}$ is related to the decomposition of the propyl chain anchored to the surface (III). Considering this as the threshold temperature for the total decomposition of the organic species supported on silica, the loss is found to be about $15 \%$ of the overall weight (temperature range between 200 and $600{ }^{\circ} \mathrm{C}$ ).

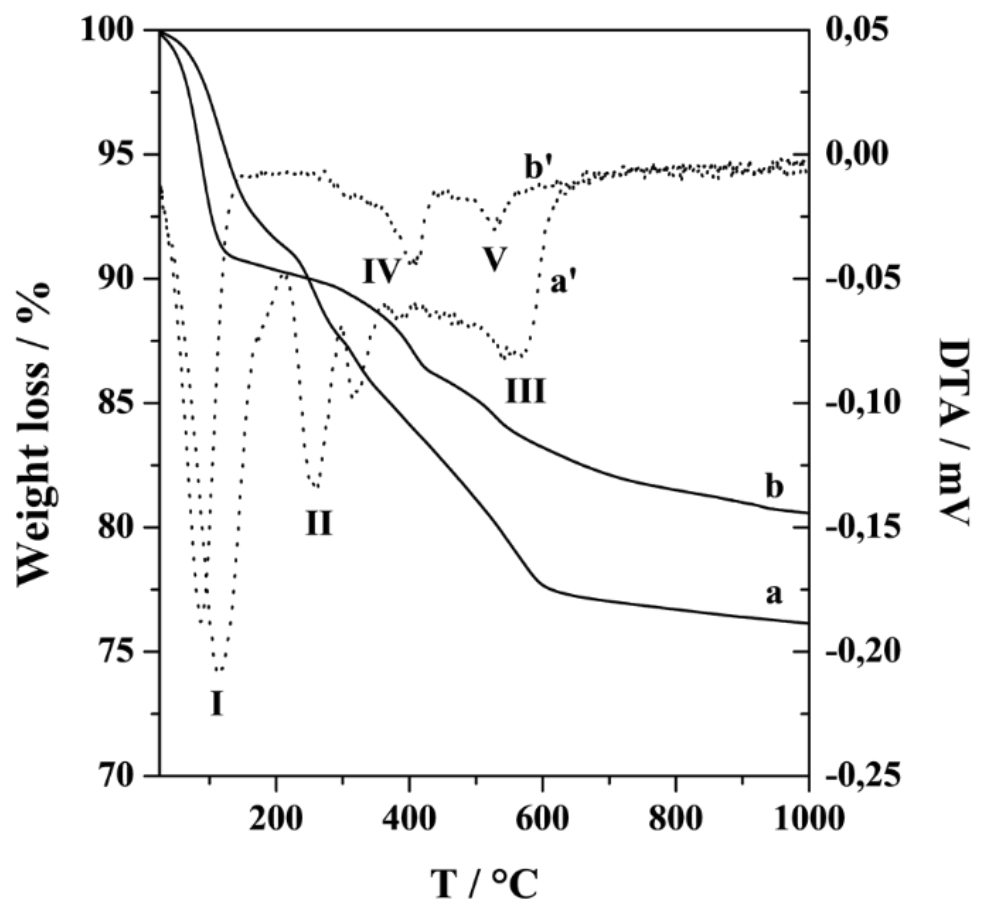

Figure 3.10: TGA and DTA curves of $\mathrm{HSO}_{3}-\mathrm{NOS}-\mathrm{H}$ (a and $\mathrm{a}^{\prime}$ ) and $\mathrm{HSO}_{3}$ - NOS-F (b and $\left.\mathrm{b}^{\prime}\right)$. 
Shifting to $\mathrm{HSO}_{3}-\mathrm{NOS}-\mathrm{F}$ (curves $\mathrm{b}$ and $\mathrm{b}^{\prime}$ ), after the first loss due to the desorption of physisorbed water, a main weight loss due to the organic moieties is found at about 300$450{ }^{\circ} \mathrm{C}$. This is related to the breakage of the ether bond linking the propyl chain to the fluorinated phenyl ring (IV). Finally, the weight loss between 500 and $600{ }^{\circ} \mathrm{C}$ is ascribed to the thermal decomposition of the alkyl chains, still bounded to the silica surface (V). The weight loss due to the organic species is about $8.4 \%$ of the total weight. In both hybrid solids, a progressive weight loss at temperatures above $600{ }^{\circ} \mathrm{C}$ is observed, due to dehydroxylation phenomena, i.e. condensation of external silanols with consequent release of water molecules and formation of strained siloxane bridges at high temperatures. The TGA results evidenced that probably the thermal stability of the urea group is slightly lower than that of the ether groups, with the $\mathrm{HSO}_{3}-\mathrm{NOS}-\mathrm{H}$ sample being less stable than $\mathrm{HSO}_{3}$-NOS-F, from a thermal point of view.

After studying the amount and the stability of the organic species constituting the hybrid materials, the focus was shifted to the textural properties. $\mathrm{N}_{2}$ physisorption analysis at $77 \mathrm{~K}$ was conducted on the two hybrid materials and on a pure silica sample synthesized with the same methodology, for comparison, in order to obtain information on specific surface area and free pore volume of the catalysts, as well as on the effect of the presence of the $\mathrm{R}-\mathrm{Si}\left(\mathrm{OR}^{\prime}\right)_{3}$ silylated precursor in the synthesis mixture (Fig. 3.11).

The isotherms show a type IV behavior (IUPAC), with a hysteresis loop typical of conventional mesoporous materials. The $\mathrm{N}_{2}$ uptake is also consistent with mesoporous materials obtained through a fluoride-catalyzed sol-gel approach. Furthermore, hysteresis and sharp peaks in the desorption branch could be due cavitation phenomena and an inkbottle pore shape. ${ }^{78}$ To obtain information about the surface area of the catalysts, the Brunauer-Emmett-Teller (BET) method was implemented. Both organo-catalysts show a surface area comparable to that of the pure silica reference material $\left(802 \mathrm{~m}^{2} / \mathrm{g}\right)$. Specifically, $\mathrm{HSO}_{3}$-NOS-H shows a surface area of $683 \mathrm{~m}^{2} / \mathrm{g}$ whereas $\mathrm{HSO}_{3}-\mathrm{NOS}-\mathrm{F}$ exhibits an area of $816 \mathrm{~m}^{2} / \mathrm{g}$. The slightly lower value observed for the non-fluorinated catalyst may be related to the usage of a bulkier organosilane precursor in the one-pot synthesis of the solid. The adsorption branches of the isotherms were analyzed by means of the NLDFT (non-localized density functional theory) and the Barrett-Joyner-Halenda (BJH) methods to obtain more detailed information on the pore-size distributions. 

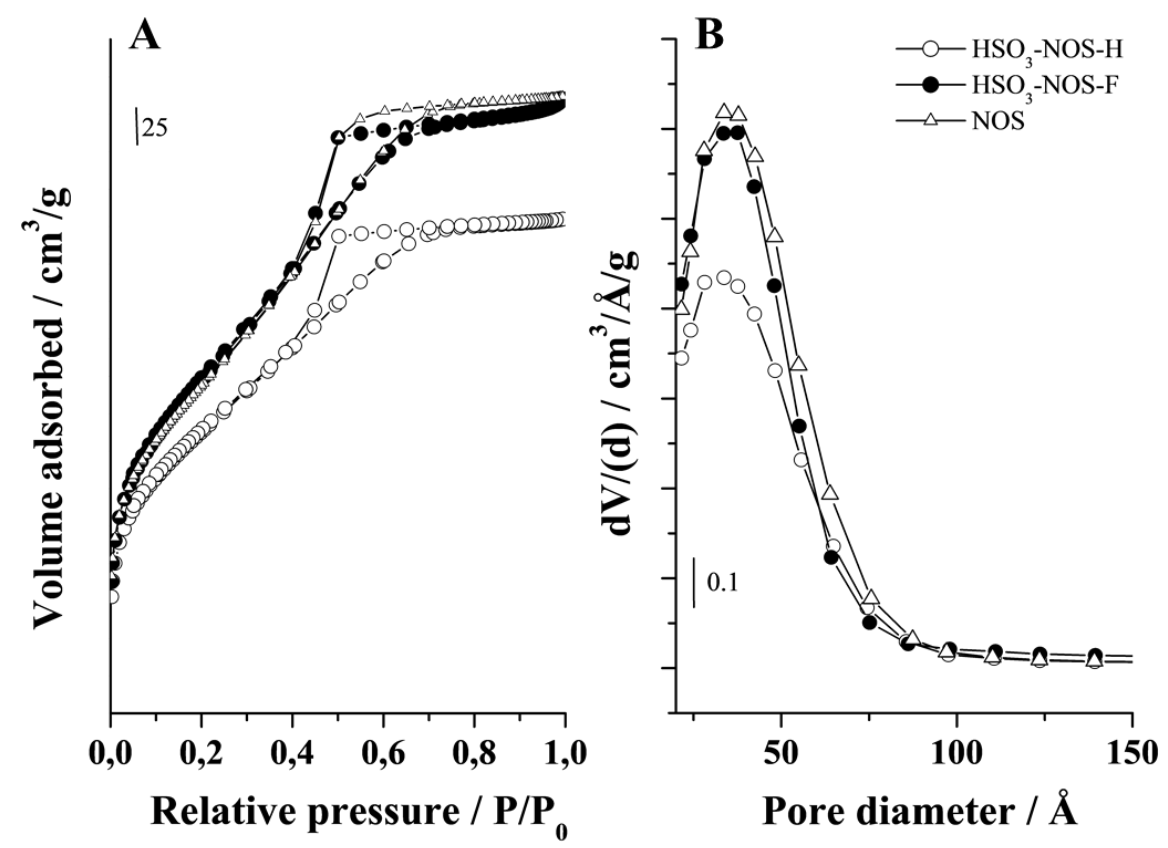

Figure 3.11 A: $\mathrm{N}_{2}$ adsorption/desorption isotherms at $77 \mathrm{~K}$ of $\mathrm{HSO}_{3}-\mathrm{NOS}-\mathrm{H}, \mathrm{HSO}_{3}-\mathrm{NOS}-\mathrm{F}$ hybrid materials and non-ordered silica (NOS, reference material). B: Pore-size distribution of the materials in the mesopore range.

Both functionalized non-ordered silicas and the reference pure silica material show a pore distribution spacing between 20 and $60 \AA$ with a peak centered at about $35 \AA$, typical of non-ordered silica materials obtained through a fluoride-catalyzed sol-gel process. ${ }^{78}$ In all three samples, a small amount of micropores is present. This has already been observed in other syntheses carried out in $\mathrm{NH}_{4} \mathrm{~F}$ medium. ${ }^{46,78}$ After exploring the amount and stability of the organic moieties and the textural properties of the hybrid materials, FTIR and solid state NMR spectroscopic characterization was carried out to address the organic-inorganic interaction and to study the acidic properties of the hybrids. ${ }^{79}$ All samples on which FTIR spectra were recorded were previously outgassed for $2 \mathrm{~h}$ at $453 \mathrm{~K}$ to remove physisorbed water.

The $\mathrm{HSO}_{3}$-NOS-H spectrum (Fig. 3.12, curve c) appears quite different compared with the pure silica reference material, NOS (Fig. 3.12, curve a). 


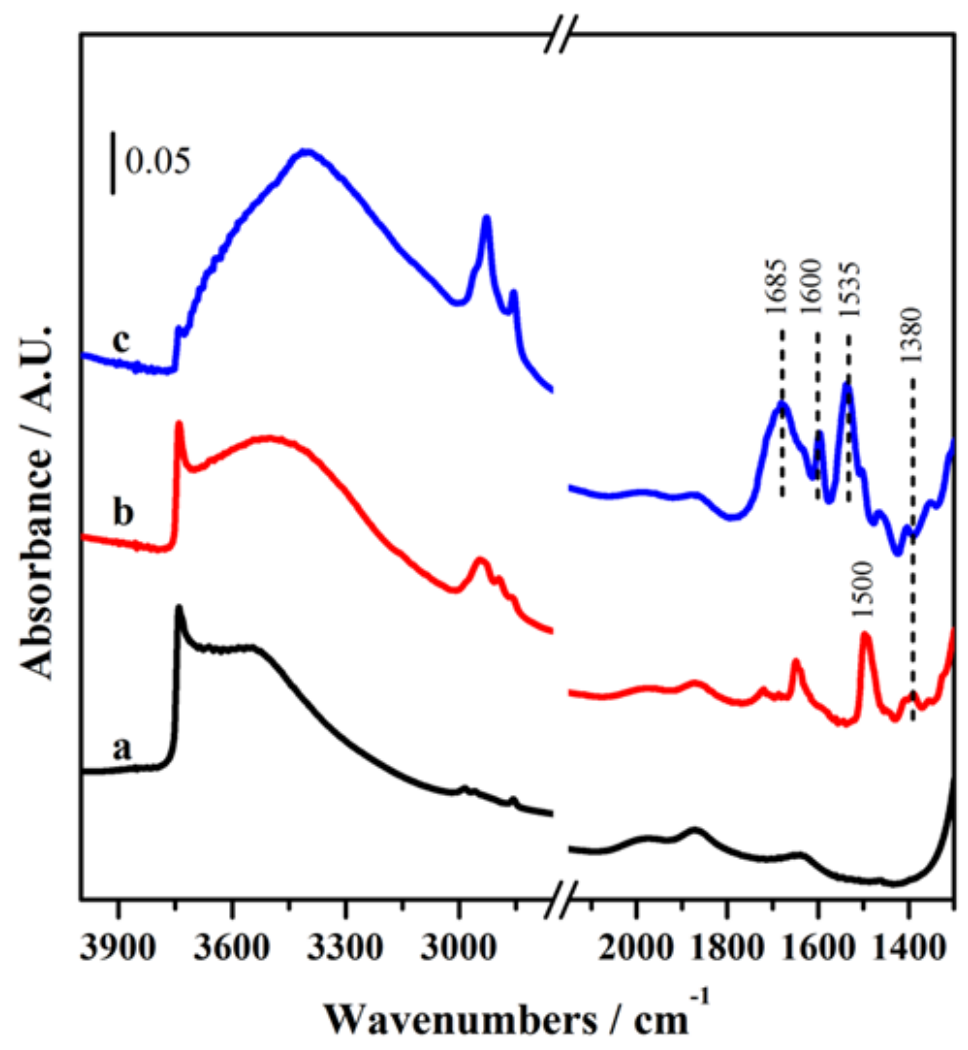

Figure 3.12: FTIR spectra of non-ordered silica (NOS, curve a), $\mathrm{HSO}_{3}-\mathrm{NOS}-\mathrm{F}$ (curve b) and $\mathrm{HSO}_{3}-\mathrm{NOS}-\mathrm{H}$ (curve c). Samples outgassed at $453 \mathrm{~K}$ before recording the spectra.

The presence of the bulky silyl-derivative in the synthesis led to a reduced amount of isolated silanols in the final material $\left(\mathrm{HSO}_{3}-\mathrm{NOS}-\mathrm{H}\right)$. Specifically, a signal centered at $3745 \mathrm{~cm}^{-1}$, due to the $\mathrm{O}-\mathrm{H}$ stretching mode of $\mathrm{Si}-\mathrm{OH}$ groups, is visible, although with much lower intensity than in the reference material. Instead, a broad signal related to silanols interacting with each other through a H-bond is observed between 3500 and $3200 \mathrm{~cm}^{-1}$. Multiple signals related to the stretching mode of $\mathrm{C}-\mathrm{H}$ groups present in the aromatic ring, in the propyl linker and in eventual uncondensed alkoxy groups, are observed between 3000 and $2800 \mathrm{~cm}^{-1}$. The low frequency region appears quite rich in signals, due to the presence of the urea group, which is characterized by many active vibrations. The most typical ones are the signal centered at $1685 \mathrm{~cm}^{-1}$, attributed to the stretching modes of the $\mathrm{C}=\mathrm{O}$ bond of the urea group, and the amide II band, centered at 
$1600 \mathrm{~cm}^{-1}$, that is related to a motion combining the $\mathrm{N}-\mathrm{H}$ bending and $\mathrm{C}-\mathrm{N}$ stretching of the amide group. ${ }^{80}$ The sharp band at $1535 \mathrm{~cm}^{-1}$ is ascribed to the $\mathrm{C}-\mathrm{C}$ stretching of the aromatic ring. Additionally, a weak band at $1380 \mathrm{~cm}^{-1}$ is observed and associated to the $\mathrm{S}=\mathrm{O}$ asymmetric stretching mode of sulfonic groups.

Shifting the focus to the $\mathrm{HSO}_{3}$-NOS-F FTIR spectrum (Fig. 3.12, curve b), it can be observed that by implementing a less bulky silyl-derivative as an organosilane precursor, the amount of isolated silanols $\left(3745 \mathrm{~cm}^{-1}\right)$ in the resulting material appears comparable to that of the pure silica one. A composed signal centered between 3000 and $2800 \mathrm{~cm}^{-1}$ related to the stretching mode of $\mathrm{C}-\mathrm{H}$ groups present in the propyl chains (and in uncondensed alkoxy groups) is observed. Due to the simpler linking group present between the propyl chain and the aryl-sulfonic moieties, in this spectrum, the low frequency region shows less signals, the most notable of which is a sharp band centered at $1500 \mathrm{~cm}^{-1}$, related to the $\mathrm{C}-\mathrm{C}$ stretching of the fluorinated aromatic ring.

Multi-nuclear magic angle spinning nuclear magnetic resonance (MAS NMR) spectroscopic experiments have been carried out to study the hybrid organic-inorganic mesoporous silica-based catalysts. ${ }^{29} \mathrm{Si}$ NMR spectroscopy was implemented to study the successful incorporation of aryl-sulfonic acid groups in the framework of non-ordered mesoporous silicas. The integrity of the organic components within the hybrid catalysts' structure was explored by ${ }^{13} \mathrm{C}$ NMR spectroscopy. Further information on nature of the organic species present in the catalyst was achieved by implementing various ${ }^{1} \mathrm{H}$ MAS NMR methods, which helped to determine the proton environments as well as the acidic nature of the catalysts. The ${ }^{29} \mathrm{Si}$ speciation as well as the organosilane functionalization in different hybrid catalysts was studied by ${ }^{29} \mathrm{Si} \mathrm{CP} / \mathrm{MAS}$ NMR.

In Fig. $3.13 \mathrm{C}$, the ${ }^{29} \mathrm{Si} \mathrm{CP} / \mathrm{MAS}$ NMR spectra of the hybrid catalysts are reported. Both $\mathrm{Q}$ and T Si sites are present (Fig. 3.13 A shows what this nomenclature refers to) ${ }^{79}$ There are three different $\mathrm{Q}$ sites, namely $\mathrm{Q}^{4}\left(\mathrm{Si}(\mathrm{OSi})_{4}\right)$ sites $(-110 \mathrm{ppm}), \mathrm{Q}^{3}\left(\mathrm{Si}(\mathrm{OSi})_{3} \mathrm{OH}\right)$ sites $(-100 \mathrm{ppm})$ and $\mathrm{Q}^{2}\left(\mathrm{Si}(\mathrm{OSi})_{2}(\mathrm{OH})_{2}\right)$ sites $(-90 \mathrm{ppm})$ observed in the hybrid catalysts and related to silicon units present in the non-ordered mesoporous silica support. Additionally, the signals from $\mathrm{T}^{3}\left(\mathrm{RSi}(\mathrm{OSi})_{3}\right)$ sites $(-66 \mathrm{ppm})$ and $\mathrm{T}^{2}\left(\mathrm{RSi}(\mathrm{OSi})_{2} \mathrm{OH}\right)$ sites $(-58$ $\mathrm{ppm}$ ) confirm the presence of the organosilane groups and their successful inclusion into the porous architecture of the final hybrid catalyst. ${ }^{46}$

${ }^{13}$ C CPMAS NMR spectra of the as-synthesized hybrid catalysts are shown in Fig. 3.13 D. The assignments of the spectral resonances are based on either software predicted computed spectra or on ${ }^{13} \mathrm{C}$ liquid NMR (Fig. $3.13 \mathrm{~B}$ ). ${ }^{13} \mathrm{C} \mathrm{CP} / \mathrm{MAS}$ NMR spectra of the hybrid catalysts show the typical ${ }^{13} \mathrm{C}$ signals associated with organosilane groups confirming the preservation of the organic moieties throughout the synthesis process. On the other hand, the incomplete hydrolysis of alkoxy groups from the precursors is clearly evident through the presence of signals at around 15,50 and $59 \mathrm{ppm}$. Without any protons directly attached to the phenyl carbons, the signals related to aromatic resonance in $\mathrm{HSO}_{3}$-NOS-F material appears with low intensity (110 to $145 \mathrm{ppm}$ ). 
A<smiles>C[Si](O)(O)O[Si]O</smiles>

B
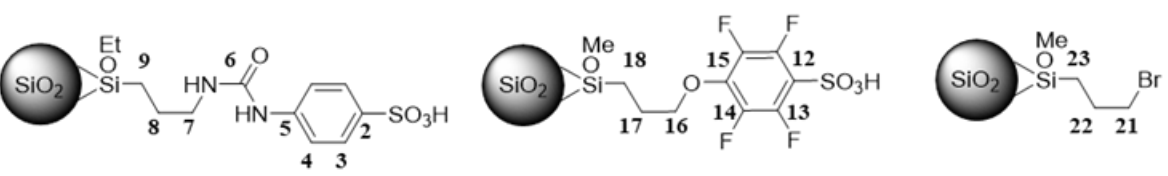

C

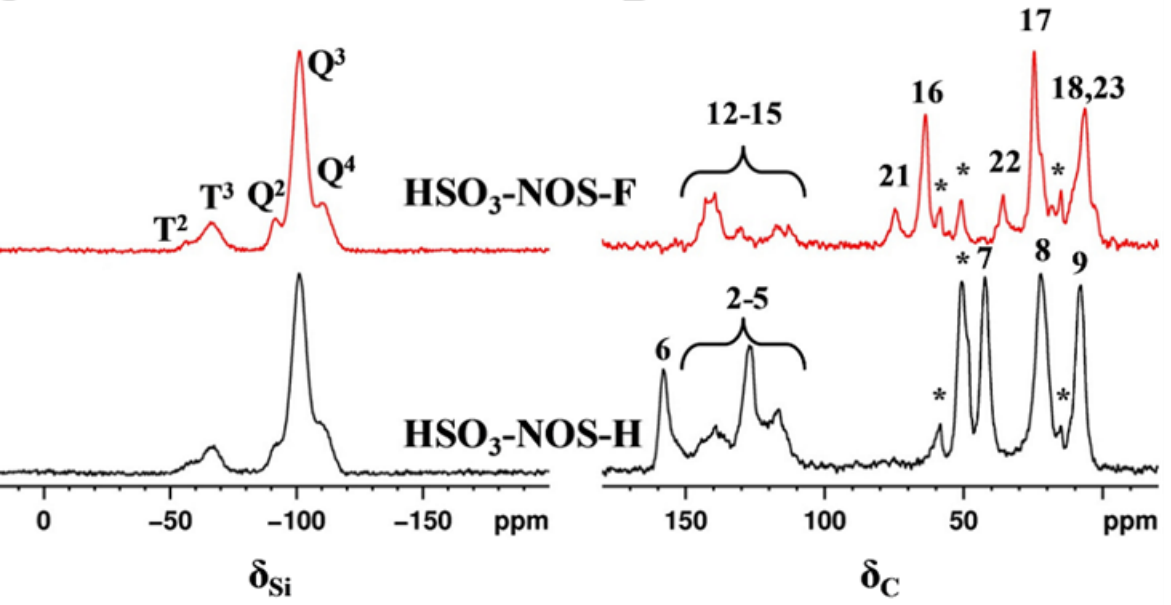

Figure 3.13 A: Schematic representation of $Q$ and $\mathrm{T}$ silicon sites detected in ${ }^{29} \mathrm{Si}$ NMR. B: Assignation of ${ }^{13} \mathrm{C}$ NMR signals of the hybrids. C: ${ }^{29} \mathrm{Si}$ CP/MAS NMR spectra of the hybrids. D: ${ }^{13} \mathrm{C}$ CP/MAS NMR spectra of the hybrids. CP contact time of $4 \mathrm{~ms}$ and $20 \mathrm{~ms}$ were used for samples $\mathrm{HSO}_{3}$-NOS-H (red curves) and $\mathrm{HSO}_{3}$-NOS-F (black curves), respectively. Signals related to uncondensed alkoxy groups are marked with *.

In fact, the intensity of ${ }^{13} \mathrm{C}$ resonances strongly depends on the proton-carbon dipolar interactions in a CP/MAS experiment. Lastly, the presence of unreacted 3-bromopropyl silane was detected in $\mathrm{HSO}_{3}$-NOS-F material. Interestingly, both spectra show signals related to uncondensed methoxy- and ethoxy groups, even if $\mathrm{HSO}_{3}-\mathrm{NOS}-\mathrm{F}$ was prepared from a trymethoxy-silyl-derivative and $\mathrm{HSO}_{3}-\mathrm{NOS}-\mathrm{H}$ was prepared from a triethoxy- 
silyl-derivative. It is likely that a fraction of the uncondensed methoxy groups in $\mathrm{HSO}_{3}$ NOS-F were exchanged to ethoxy groups during the $\mathrm{Na}^{+}$to $\mathrm{H}^{+}$exchange in acidified ethanol.

${ }^{1} \mathrm{H}$ MAS and spin-echo NMR spectra of the hybrids, previously outgassed for 90 minutes at $180{ }^{\circ} \mathrm{C}$, were recorded using a MAS rate of $15 \mathrm{kHz}$ (Fig. 3.14). Firstly, the intense resonance at $1.7 \mathrm{ppm}$ is ascribed to isolated silanols present on the surface of the silica support. The broad shoulder in the range $2-10 \mathrm{ppm}$ is attributed to hydrogen bonded silanols ${ }^{81}$ However, contributions from amine protons, sulfonic acid protons and water also fall into this broad shoulder as they are in mutually exchangeable state. Exchangeable protons undergo different binding dynamics and form structured clusters of variable sizes. These binding interactions and the proton exchange between bound water, free water, isolated $\mathrm{SiOH}$, hydrogen bonded $\mathrm{SiOH}$ and acidic/basic protons determine the chemical shift values. The heterogeneity of these interactions produces a broad range of chemical shift values, which overlap giving a broad band. In other words, this multicomponent broad signal is the weighted chemical shift of the compounds involved in the fast chemical exchange. ${ }^{82}$ Proton resonance peaks associated with organosilanes are clearly detected at around $1 \mathrm{ppm}$ for aliphatic protons and at around $7.5 \mathrm{ppm}$ for aromatic ones.

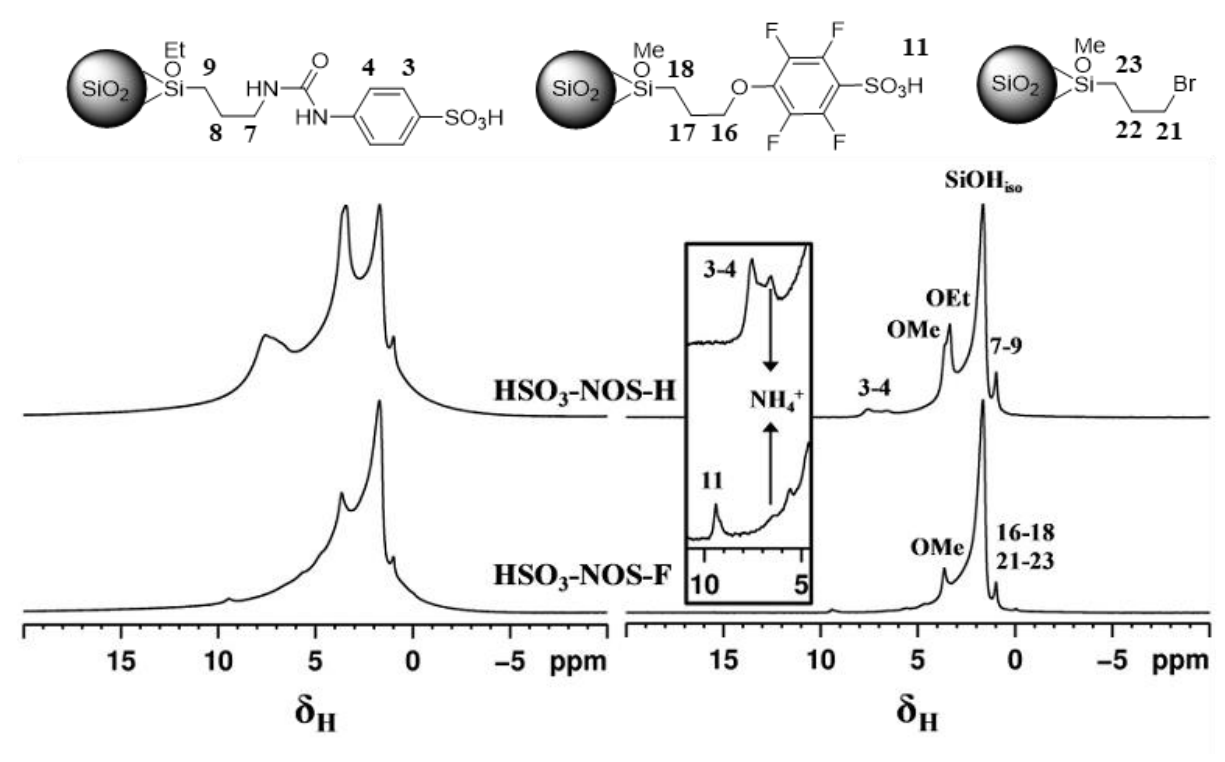

Figure 3.14: ${ }^{1} \mathrm{H}$ MAS (left panel) and ${ }^{1} \mathrm{H}$ spin-echo (right panel) NMR spectra the hybrid catalysts. Above the specta assignation of the ${ }^{1} \mathrm{H}$ signals is reported. An echo delay (tau) of $2 \mathrm{~ms}$ was used in the spin-echo experiment. Inset shows the zoomed spectra. 
No aromatic protons were detected in the $\mathrm{HSO}_{3}-\mathrm{NOS}-\mathrm{F}$ catalyst as they are substituted by fluorine. The sharp peaks in the range 3.2 to $3.6 \mathrm{ppm}$ are attributed to the alkoxy groups from the non-hydrolysed precursors. Resonance signals due to residual ammonium fluoride, used as catalyst in the synthesis of non-ordered mesoporous silica, were detected at $6.6 \mathrm{ppm}$. Lastly, the narrow low intensity resonance detected at $9.4 \mathrm{ppm}$ in the $\mathrm{HSO}_{3}$-NOS-F catalyst is attributed to sulfonic acid protons (Fig. 3.14, inset) not in exchange with other compounds.

\subsubsection{Adsorption of probe molecules followed by spectroscopies (FTIR and NMR)}

In order to study the acidity of the sulfonic groups pending from the inorganic silica surface, $\mathrm{CO}$ was adsorbed at $80 \mathrm{~K}$ on the hybrid catalysts (Fig. 3.15). Both hybrid materials behaved equally upon interaction with carbon monoxide.

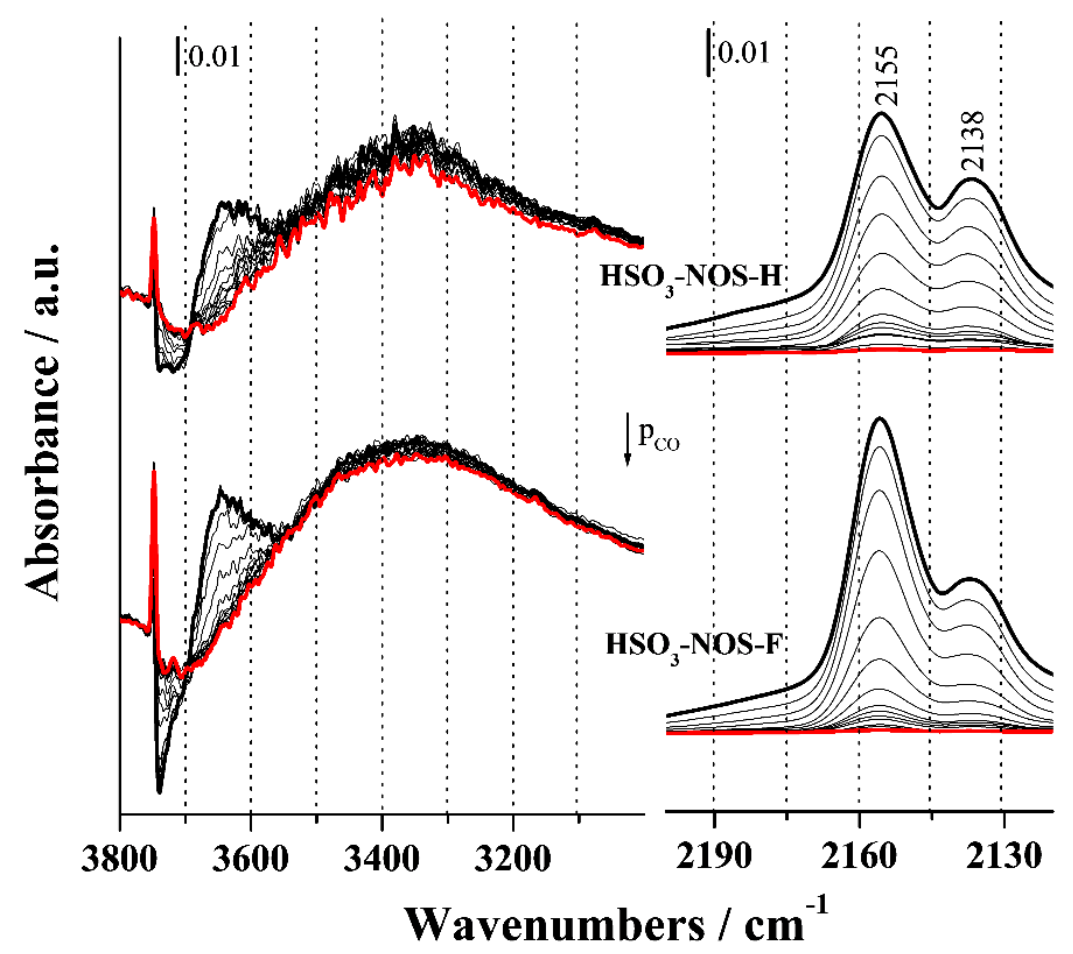

Figure 3.15: FTIR difference spectra in the $\mathrm{OH}$ (left panel) and $\mathrm{CO}$ (right panel) stretching region of $\mathrm{CO}$ adsorption at $80 \mathrm{~K}$ on $\mathrm{HSO}_{3}-\mathrm{NOS}-\mathrm{H}$ and $\mathrm{HSO}_{3}-\mathrm{NOS}-\mathrm{F}$ hybrid materials. 
Focusing on the high frequency region of the spectra, the signal related to the stretching modes of isolated silanols $\left(3745 \mathrm{~cm}^{-1}\right)$ is red-shifted, forming a broad signal centered at approximately $3644 \mathrm{~cm}^{-1}$. The $100 \mathrm{~cm}^{-1}$ frequency shift is consistent with previous studies reported on purely siliceous materials. ${ }^{76}$ At low coverages, in the $\mathrm{CO}$ stretching region, a signal centered at $2155 \mathrm{~cm}^{-1}$ is observed due to the $\mathrm{CO}$ molecules interacting with the silanol groups on the surface of the support. ${ }^{76}$ Increasing the $\mathrm{CO}$ dosages, the typical band related to the stretching mode of the free $\mathrm{CO}$ molecule appears ( $v_{\mathrm{CO}}$ liquidlike at $\left.2138 \mathrm{~cm}^{-1}\right) .83$

Given a specific site with which CO interacts, the blue shift of the stretching mode is expected to be proportional to the tendency of the site to accept electron density, i.e. its acidity. However, despite the presence of highly acidic sulfonic groups, no signal is observed at higher frequencies other than that of $\mathrm{CO}$ interacting with silanols. In other words, $\mathrm{CO}$ physisorption at $80 \mathrm{~K}$ is able to reveal only mild acidity related to the $\mathrm{Si}-\mathrm{OH}$ of the inorganic support.

After studying the interaction with a weakly basic probe molecule, a stronger base such as ammonia was adsorbed onto the hybrids. The interaction was studies by FTIR spectroscopy. For comparison, $\mathrm{NH}_{3}$ was adsorbed at room temperature on both hybrid materials and on the pure non-ordered silica support (Fig. 3.16). $\mathrm{NH}_{3}$ interaction with NOS material (Fig. $3.16 \mathrm{~A}$ ) produced features consistent with the formation of $\mathrm{H}$-bonds between $\mathrm{Si}-\mathrm{OH}$ groups of the silica and the probe molecules. ${ }^{84}$ At high $\mathrm{NH}_{3}$ pressure (30 mbar), signals at 3400 and $3320 \mathrm{~cm}^{-1}$ appear, overlapped with a broader band centered at $3020 \mathrm{~cm}^{-1}$. The band at $3745 \mathrm{~cm}^{-1}$, due to the $\mathrm{O}-\mathrm{H}$ stretching mode of free silanols (red curve), is almost completely eroded (bold black curve). The broad band centered at $3020 \mathrm{~cm}^{-1}$ is ascribed to the $\mathrm{O}-\mathrm{H}$ stretching of the silanol groups $\mathrm{H}$-bonded to $\mathrm{NH}_{3}$ molecules. The peaks at 3400 and $3320 \mathrm{~cm}^{-1}$ are due to the $\mathrm{N}-\mathrm{H}$ asymmetric and symmetric stretching modes of $\mathrm{NH}_{3}$ bonded to silanols, respectively. At lower frequency, a band at $1635 \mathrm{~cm}^{-1}$ is observed and is assigned to the asymmetric bending mode of the ammonia molecules adsorbed on $\mathrm{Si}-\mathrm{OH}$ groups. Such complexes are very weakly held and, consequently, all their spectroscopic features progressively fade away upon decreasing the $\mathrm{NH}_{3}$ pressure, and almost completely disappear after outgassing the sample for 30 minutes at room temperature (blue curve). The resulting spectrum is similar to that of the bare NOS sample before $\mathrm{NH}_{3}$ adsorption (red curve).

In contrast, ammonia adsorption on both $\mathrm{HSO}_{3}-\mathrm{NOS}-\mathrm{H}$ (Fig. $3.16 \mathrm{~B}$ ) and $\mathrm{HSO}_{3}-\mathrm{NOS}-\mathrm{F}$ (Fig. 3.16 C) hybrid materials produced, in the low frequency region, signals at 1465 and $1485 \mathrm{~cm}^{-1}$ due to the asymmetric bending mode of $\mathrm{NH}_{4}{ }^{+}$protonated species, meaning that sulfonic acid groups could protonate ammonia molecules. ${ }^{85}$ Ammonia protonation is an irreversible process; in fact, upon $\mathrm{NH}_{3}$ desorption at room temperature, the signal related to $\mathrm{NH}_{4}{ }^{+}$bending modes is still visible on both hybrid catalysts (blue curves). Difference FTIR spectra are reported in the inset, to better evidence the formation of the $\mathrm{NH}_{4}{ }^{+}$bands, partially overlapped with other signals due to the organic fragments of the hybrid solids. 

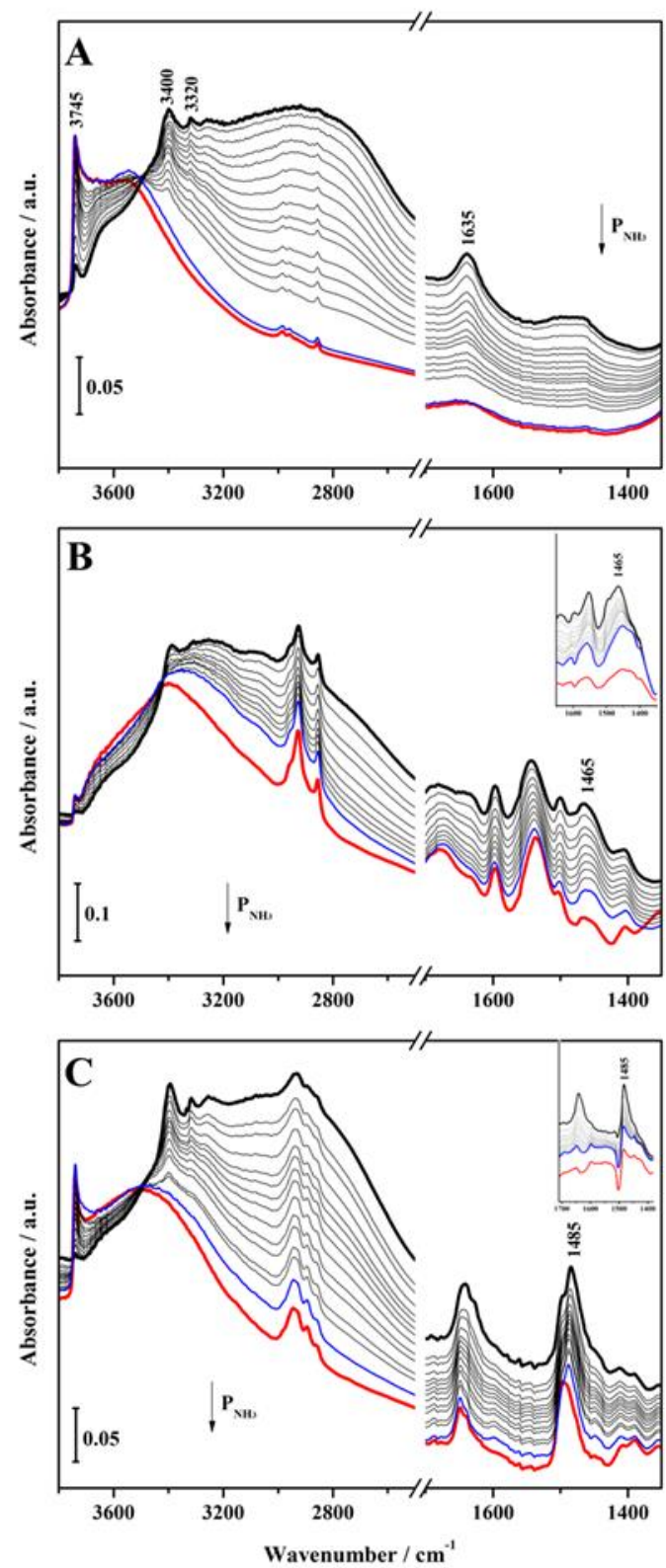

Figure 3.16: FTIR spectra of $\mathrm{NH}_{3}$ adsorption at $298 \mathrm{~K}$ on $\mathrm{NOS}(\mathrm{A}), \mathrm{HSO}_{3}-\mathrm{NOS}-\mathrm{H}(\mathrm{B}), \mathrm{HSO}_{3}$ NOS-F (C) hybrid materials. Red curves: hybrids before to $\mathrm{NH}_{3}$ adsorption. Black curves: 30 mbar $\mathrm{NH}_{3}$. Blue curves: $\mathrm{NH}_{3}$ outgassing at rt. $\mathrm{B}$ and $\mathrm{C}$ insets: FTIR difference spectra. 
${ }^{1} \mathrm{H}$ NMR spectroscopy was also implemented to study the acidity of the hybrid catalysts by adsorbing a basic probe molecule. Ammonia was adsorbed on the hybrid catalysts, previously outgassed at $180{ }^{\circ} \mathrm{C}$ for 90 minutes. ${ }^{1} \mathrm{H}$ spin-echo NMR spectra were recorded after ammonia adsorption and are shown in Fig. 3.17. The acid-base interaction between the ammonia and the pendant sulfonic groups results in a proton transfer and consequent formation of ammonium ions. This is evident from the intensity increase of the signal at $6.6 \mathrm{ppm}$, previously ascribed to residual ammonium ions implemented as catalyst in the sol-gel process. Moreover, a small decrease in the peak intensity of sulfonic acid protons is also observed in $\mathrm{HSO}_{3}$-NOS-F sample. While keeping into account non-quantitative nature of the spin-echo ${ }^{1} \mathrm{H}$ NMR spectroscopy, it is worth noticing that 4 protons contribute to the signal intensity of the ammonium ion, while only one proton contributes to the signal of a given sulfonic acid group. Consequentially, compared to the decrease experienced by the sulfonic acid signal, the signal related to the ammonium ion is expected to experience a more pronounced increase in intensity. The presence of a $9.4 \mathrm{ppm}$ peak in the spectrum after ammonia adsorption also confirms the fact that not all sulfonic acid protons are accessible to ammonia. On the other hand, despite the fact that no signal related to the sulfonic acid proton was identified in the ${ }^{1} \mathrm{H}$ spectrum of $\mathrm{HSO}_{3}-\mathrm{NOS}-\mathrm{H}$ material, a marked increase in the formation of ammonium ions after ammonia adsorption is observed.

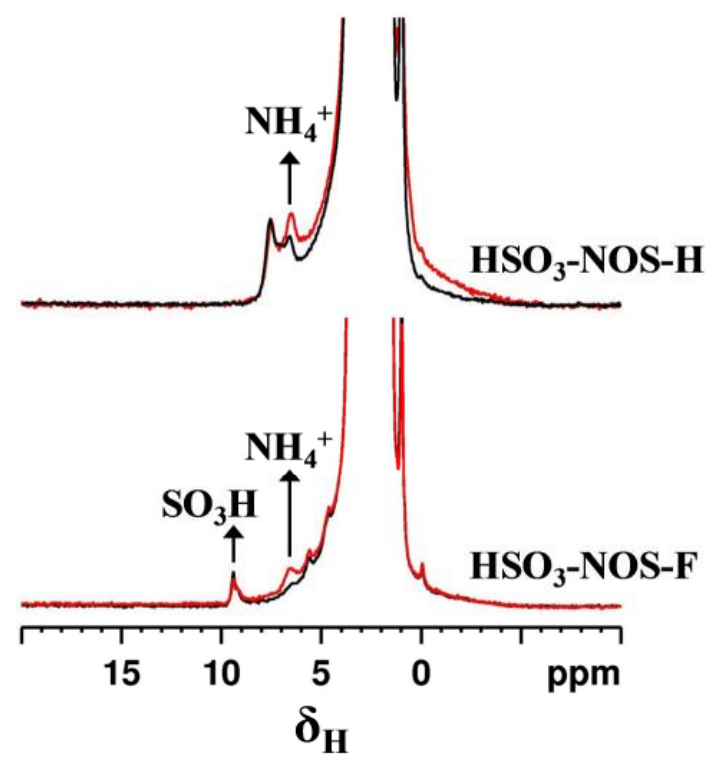

Figure 3.17: ${ }^{1} \mathrm{H}$ spin-echo NMR spectra of the hybrid catalysts, before (black) and after (red) ammonia adsorption. 
At this point of the discussion, it is worth broadening the perspective on the results of the spectroscopic characterization (FTIR and NMR), prior and after interaction of the acid catalysts with probe molecules with different basicity. Both FTIR and ${ }^{1} \mathrm{H}$ solid state NMR showed spectroscopic features related to species in H-bond interactions. It has been long known that the silanol groups present on surface of mesoporous silica supports strongly interact with each other. ${ }^{86}$ Moreover, several authors reported on the interaction occurring between the surface of a silica support and pendant functional groups (typically amines) linked together by a propyl spacer. The flexibility of the $\mathrm{C} 3$ chain allows for the functional group to undergo non-covalent interaction (H-bond) with the surface silanols. ${ }^{87}$ These interactions can explain the absence of a clear ${ }^{1} \mathrm{H}$ NMR signal related to the sulfonic protons in $\mathrm{HSO}_{3}$-NOS-H material. Due to the surface topology, each sulfonic proton can form a slightly different $\mathrm{H}$-bond interaction with the silanols in its proximity, which results in a broad spectroscopic feature; furthermore, the relative abundance of sulfonic proton in the sample is quite low. These two aspects together cause the signal to be masked by the broad signal of other H-bond interacting protons.

Weak bonds also affect the interactions of the hybrids with base probes. While after contact with of $\mathrm{CO}$ (weak base) no interaction with sulfonic acid groups was detected (FTIR, Fig 3.15), upon contact with $\mathrm{NH}_{3}$ (strong base) proton transfer occurred and an ion pair was formed (FTIR, Fig. 3.16; NMR, Fig 3.17). This is especially counterintuitive when considering that $\mathrm{CO}$ can interact with surface silanols, which are much less acidic than sulfonic acid protons. An interpretation of these results is reported below. H-bond interactions between $\mathrm{O}-\mathrm{H}$ and the acceptor $\mathrm{X}$ (resulting in the complex $\mathrm{O}-\mathrm{H} \cdots \mathrm{X}$ ) can be described as transfer of part of the electron density of the $\mathrm{O}-\mathrm{H}$ bond to the new $\mathrm{H} \cdots \mathrm{X}$ weak bond. This can be observed in the FTIR red-shift (i.e. shift toward lower energy) that occurs when $\mathrm{O}-\mathrm{H}$ groups interact through $\mathrm{H}$-bond with each other or with other species ${ }^{88} \mathrm{H}$-bond interactions and reversible and can occur whenever they have a stabilizing effect on the system, lowering the overall free energy. Considering the hybrid catalysts described in this Chapter, breaking the interactions between sulfonic acid groups and surface silanols would require a species $\mathrm{X}$ able to form an $\mathrm{H} \cdots \mathrm{X}$ complex more energetically favorable than the previous complex (Fig. 3.18). Given the very weak basicity of carbon monoxide, it is reasonable to think that it might not be a good enough $\mathrm{H}$-bond acceptor to break the interaction between sulfonic acids and silanols. Nevertheless, upon contact with a much stronger base (i.e. ammonia), a full acid-base interaction can occur, which lowers the energy of the system much more than the original $\mathrm{H}$-bond. 


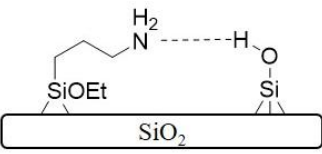

B

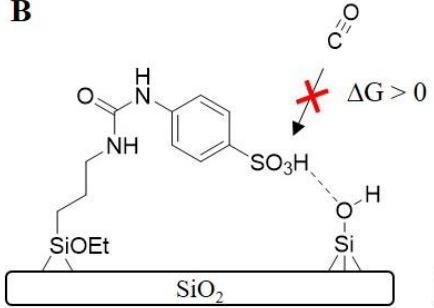

C

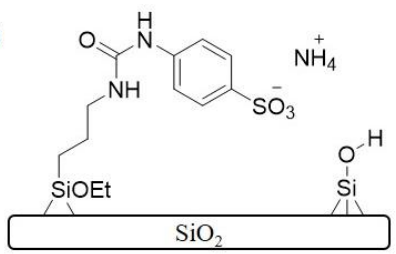

Figure 3.18: Schematic representation of the interaction between the silica surface and $\mathrm{NH}_{2}$ pr groups ${ }^{87}(\mathrm{~A})$, sulfonic acid groups in $\mathrm{HSO}_{3}-\mathrm{NOS}-\mathrm{H}$ material in presence of $\mathrm{CO}(\mathrm{B})$ and ammonia $(\mathbf{C})$.

The high speciation of protons in the sample poses limitations to the extent to which the acidic properties of the hybrid catalysts can be studied. To this scope, phosphorous-based probe molecules have been implemented by several authors. ${ }^{89}$ Through ${ }^{31}$ P MAS NMR, the chemical shift of the electron-rich atom in the probe molecule can be measured, without the contribution of any species other than the probe molecules. In this work, trimethylphosphine oxide (TMPO) is used. ${ }^{90,91}$ In Fig. 3.19 A, the room temperature ${ }^{31} \mathrm{P}$ MAS NMR spectra of $\mathrm{HSO}_{3}-\mathrm{NOS}-\mathrm{H}$ and $\mathrm{HSO}_{3}-\mathrm{NOS}-\mathrm{F}$ hybrid materials after contact with TMPO adsorption are reported.
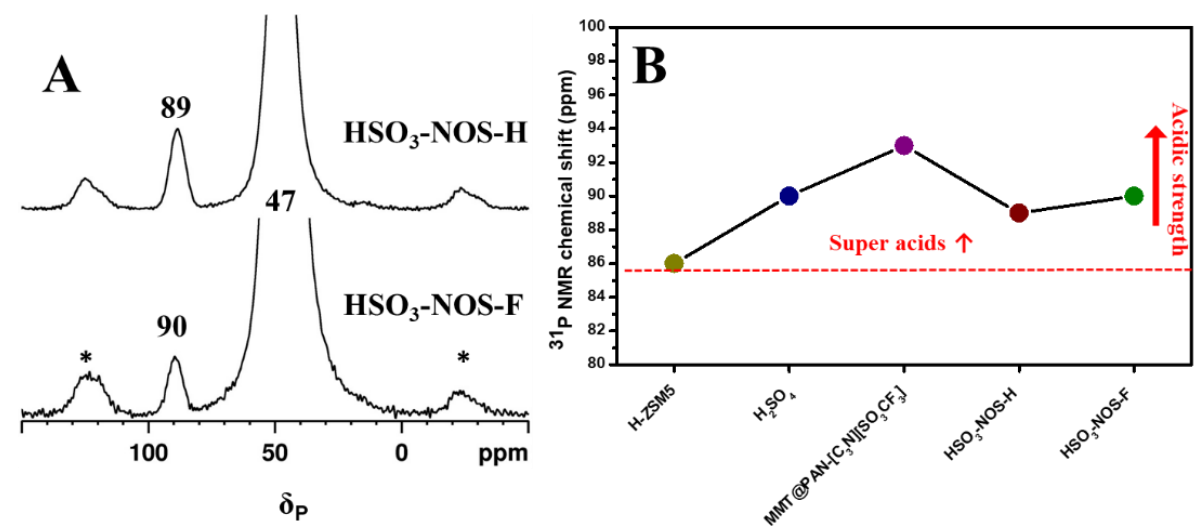

Figure 3.19 A: ${ }^{31} \mathrm{P}$ MAS NMR spectra of the hybrid catalysts recorded at room temperature. * refers to spinning sidebands. B: Acid strength versus ${ }^{31} \mathbf{P}$ MAS NMR chemical shifts of various super acids catalysts reported in the literature (ref. 93). 
Upon interaction with the hybrids, the ${ }^{31} \mathrm{P}$ NMR signal of TMPO molecule was downfield shifted, proportionally to the acid strength of the material. ${ }^{92}$ Both samples exhibit an intense peak at $47 \mathrm{ppm}$, ascribed to physisorbed TMPO molecules. The resonance peaks at 89 and 90 ppm in $\mathrm{HSO}_{3}-\mathrm{NOS}-\mathrm{H}$ and $\mathrm{HSO}_{3}-\mathrm{NOS}-\mathrm{F}$ materials, respectively, confirm the very strong acidity displayed by these hybrids. A recent article has charted the acid strength of super acid catalysts using ${ }^{31} \mathrm{P}$ MAS NMR spectroscopy. The highest chemical shift for TMPO was reported to be $93 \mathrm{ppm}$ for a sulfonated polymer embedded in montmorillonite ${ }^{89,93}$ Based on this report, $\mathrm{HSO}_{3}-\mathrm{NOS}-\mathrm{H}$ and $\mathrm{HSO}_{3}-\mathrm{NOS}-\mathrm{F}$ hybrid materials display acid strength similar to that of $100 \% \mathrm{H}_{2} \mathrm{SO}_{4}$ (Fig. $3.19 \mathrm{~B}$ ).

\subsubsection{Catalytic activity}

The catalytic activity of the $\mathrm{HSO}_{3}-\mathrm{NOS}-\mathrm{H}$ and $\mathrm{HSO}_{3}-\mathrm{NOS}-\mathrm{F}$ hybrid catalysts was evaluated in the acetal formation reaction between benzaldehyde and ethylene glycol (Fig. $3.20)$.

$\mathbf{A}$

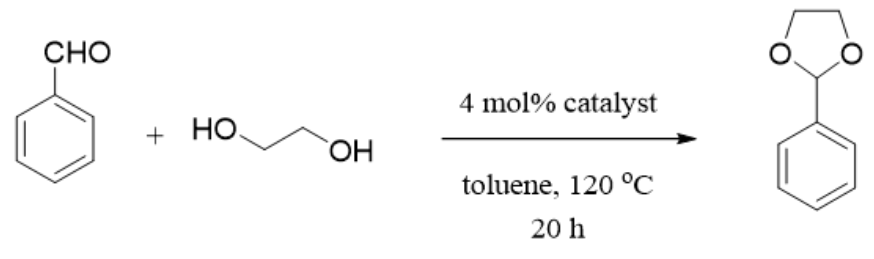

B

- Reaction $\quad$ Recycle $1 \quad$ Recycle 2

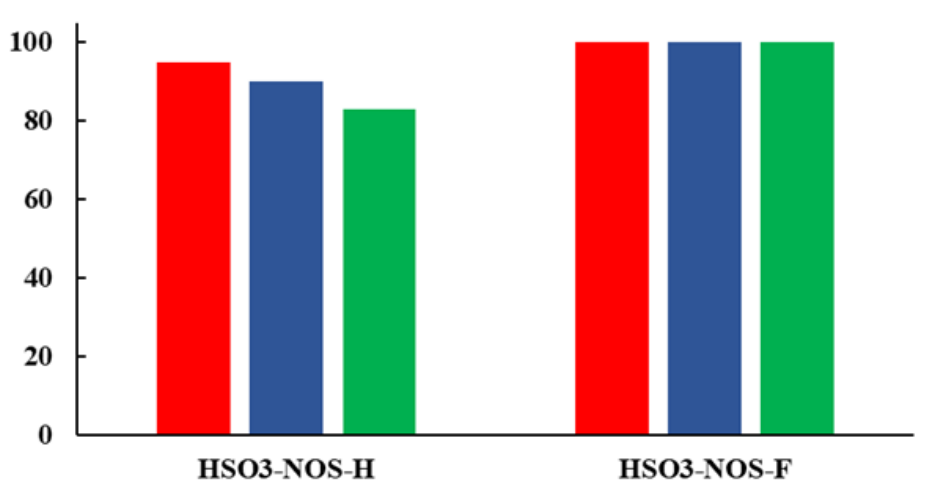

Figure 3.20 A: Schematic representation of the acetal formation reaction between benzaldehyde and ethylene glycol. B: Yields obtained using catalysts $\mathrm{HSO}_{3}-\mathrm{NOS}-\mathrm{H}$ and $\mathrm{HSO}_{3}-\mathrm{NOS}-\mathrm{F}$. 
This reaction is particularly interesting to synthetic organic chemists, as it is used to protect aldehydes and ketones, stabilizing them under basic reaction conditions, in order to facilitate subsequent reduction and oxidation transformations. ${ }^{94,95}$ Preliminary catalytic findings, reported in Fig. 3.20, show promising catalytic trends in both reactivity and recyclability, confirming the potential of these type of heterogeneous catalysts. $\mathrm{HSO}_{3}-\mathrm{NOS}-\mathrm{F}$ catalyst demonstrates a slightly higher stability, with high quantitative yields and recyclability under the reaction conditions implemented. These findings prove the robustness of the anchoring strategy. Under the same reaction conditions, catalyst $\mathrm{HSO}_{3}-\mathrm{NOS}-\mathrm{H}$ shows a slightly lower activity, probably due to its lower acidity. The differences observed are ascribed to the presence of fluorine atoms in the benzene ring of $\mathrm{HSO}_{3}-\mathrm{NOS}-\mathrm{F}$ catalyst. Highly electronegative fluorine atoms withdraw electron density from the aromatic ring, therefore making it more electron-poor. Consequently, electron density is withdrawn also from the sulfonic group, attached to the aromatic ring ultimately making the release of a proton more favorable (i.e. making it more acidic). The scope of the acetalization reaction was also investigated with different ketones and aldehydes using $\mathrm{HSO}_{3}-\mathrm{NOS}-\mathrm{H}$ catalyst to evaluate the wider potential of this anchored catalyst containing pendant acid sites (Table 3.1).

Promising results were obtained when electron-poor benzaldehydes and cyclohexanone were employed as substrates. As expected, electron-rich aldehydes represent a bigger challenge due to their electronic nature and require longer reaction times or harsher reaction conditions. To evaluate wider industrial applicability, the effectiveness of this anchored catalyst in the transacetalization reaction was also tested. Promising results were achieved when dimethoxy propane was used (60\% yield). Beyond the scope of this work, further catalytic testing would be needed to better rationalize the potential and applicability of the hybrid catalysts, in view of a possible industrial application. 
Table 3.1: Evaluation of the scope of the anchored catalyst, $\mathrm{HSO}_{3}-\mathrm{NOS}-\mathrm{H}$, for acetalization and transacetalization reactions.
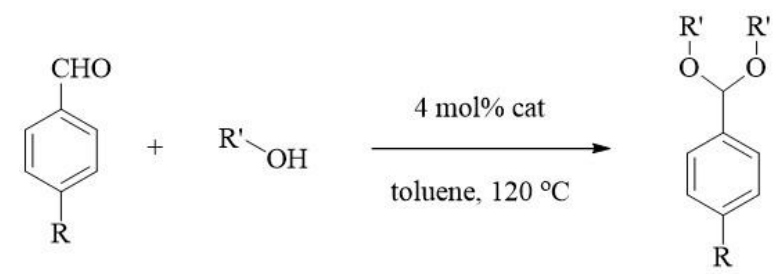

General reaction

\begin{tabular}{|c|c|c|}
\hline Aldehyde/ketone & Alcohol & Yield \% \\
\hline $\mathrm{CHO}$ & & 56 \\
\hline $\mathrm{R}=\mathrm{NO}_{2}$ & & 100 \\
\hline $\mathrm{R}=\mathrm{OMe}$ & & 10 \\
\hline & & 100 \\
\hline $\mathrm{OMe}$ & & 60 \\
\hline $\mathrm{R}=\mathrm{NO}_{2}$ & $\mathrm{CH}_{3} \mathrm{OH}$ & 16 \\
\hline
\end{tabular}

\subsubsection{Hydrophobic $\mathrm{HSO}_{3}-\mathrm{CH}_{3} \mathrm{NOS}-\mathrm{F}$ and $\mathrm{HSO}_{3}-\mathrm{CH}_{3} \mathrm{NOS}-\mathrm{F}$ hybrid materials}

In order to study the effect of the surface in promoting/hindering the catalytic activity of the supported sulfonic acids, passivated counterparts of the hybrid catalysts so far described were prepared (Fig. 3.21). To achieve this, the silanol groups on the surface of the silica support were reacted with hexamethyl disilazane (HMDS), leading to a material with methylated pore walls. For the synthesis of $\mathrm{HSO}_{3}-\mathrm{CH}_{3} \mathrm{NOS}-\mathrm{H}$ material, hybrid 3 was dehydrated at $150{ }^{\circ} \mathrm{C}$, under $\mathrm{N}_{2}$ atmosphere, and then reacted with HMDS for $2 \mathrm{~h}$, in DMSO. The resulting material was exchanged with $\mathrm{HCl}$ in ethanol to achieve the protonic form. Surprisingly, applying the same synthetic approach to the fluorinated hybrid caused leaching of a significant fraction of the pendant organic moieties. For that, an alternative approach was designed, in which hybrid 4 was reacted with HMDS, under the same synthetic conditions implemented for the non-fluorinated hybrid solid. 
A
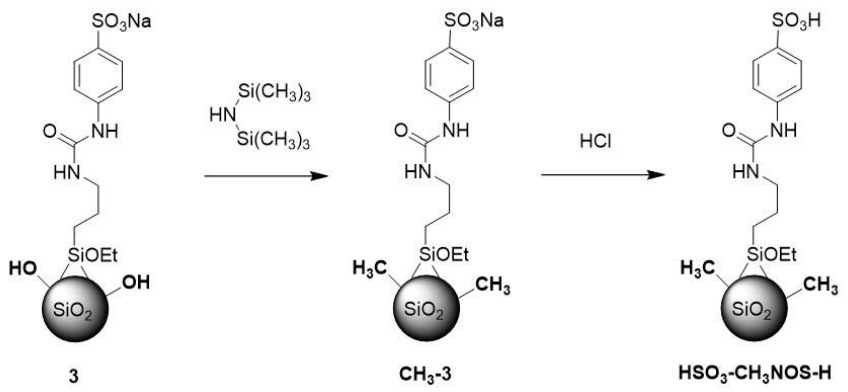

B
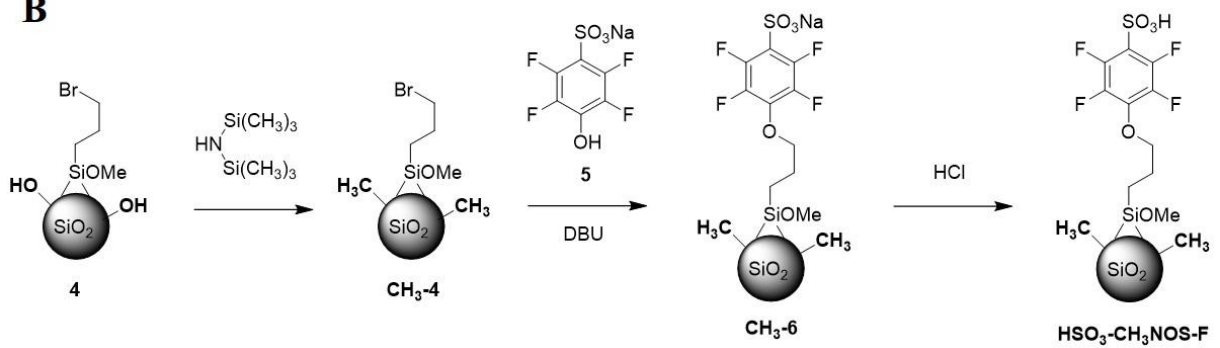

Figure 3.21: Schematic representation of the synthesis of hydrophobic silica-supported sulfonic acid catalysts.

In a second step, the tethering reaction between the pendant Br-propyl groups and compound 5 was carried out, under the same conditions used for the synthesis of hybrid $\mathrm{HSO}_{3}$-NOS-F. Lastly, the sample was washed with $\mathrm{HCl}$ in ethanol, to achieve the proton form.

To evaluate the effect of the passivation strategy, catalytic tests in the acetal formation reaction between benzaldehyde and ethylene glycol were performed (Fig. 3.22). Both hybrid materials show promising catalytic activity, exhibiting results that resemble those observed using the non-passivated solids as catalysts. Nevertheless, focusing on the third catalytic cycle a more pronounced drop in the catalytic performances is observed for the passivated non-fluorinated hybrid material $\left(\mathrm{HSO}_{3}-\mathrm{CH}_{3} \mathrm{NOS}-\mathrm{H}\right)$, compared with the nonpassivated non-fluorinated sample $\left(\mathrm{HSO}_{3}-\mathrm{NOS}-\mathrm{H}\right)$. Moreover, the same trend is observed with the passivated fluorinated sample, $\mathrm{HSO}_{3}-\mathrm{CH}_{3} \mathrm{NOS}-\mathrm{F}$, showing a slight drop in its performances, although much less pronounced than that observed for $\mathrm{HSO}_{3}$ $\mathrm{CH}_{3} \mathrm{NOS}-\mathrm{H}$ sample. 
A

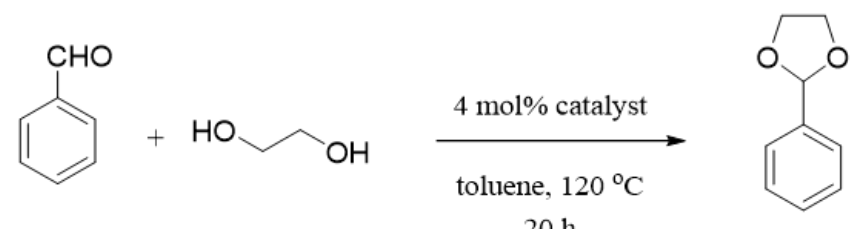

$20 \mathrm{~h}$

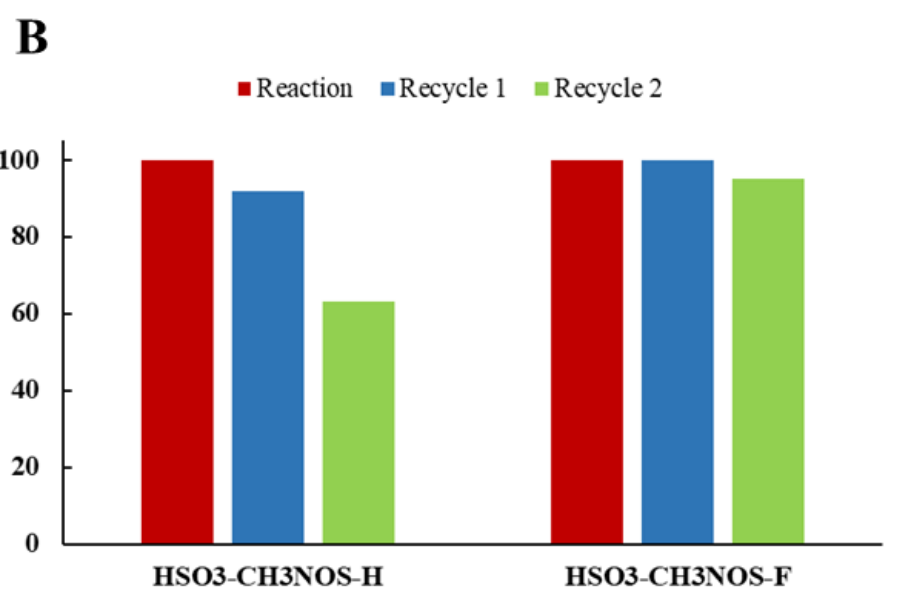

Figure 3.22 A: Schematic representation of the acetal formation reaction between benzaldehyde and ethylene glycol. B: Yields obtained for acetalization reactions using $\mathrm{HSO}_{3}-\mathrm{CH}_{3} \mathrm{NOS}$ $\mathrm{H}$ and $\mathrm{HSO}_{3}-\mathrm{CH}_{3} \mathrm{NOS}-\mathrm{F}$ catalysts.

From these preliminary catalytic tests two main observations can be made. Firstly, the beneficial influence of a fluorinated aromatic ring is confirmed and remains valid also when the surface of the inorganic support is passivated. Secondly, capping the surface silanol groups seems to affect the catalytic activity of the hybrid materials in a negative fashion.

A possible explanation can be found by observing the polarity of the substrates implemented, i.e. benzaldehyde and ethylene glycol. When substrates are non-polar, such as in biodiesel production, hydrophobic pore walls favors mass transfer and adsorption of the substrates, ultimately boosting catalytic activity. ${ }^{17,37,39}$ On the other hand, if the substrates are polar, such as in the reaction herby reported, it should be expected that the presence of polar groups on the pore wall would have the same beneficial effect. Accordingly, silanol capping would hinder mass transfer and substrate adsorption, with a detrimental effect on the overall catalytic activity. Further investigation based on this hypothesis might involve (i) testing the catalysts in lower quantities, in the attempt of 
achieving yield of about $10 \%$, as this would allow to better appreciate slight differences in the catalytic activity from the first catalytic cycle, (ii) evaluation of the quantity of substrates that remains adsorbed onto the catalysts after the reactions and (iii) spectroscopic studies of the interaction occurring between the surface of the catalysts and the reaction substrates.

Although interesting from an academic point of view, further catalytic evaluations of the less promising passivated catalyst were beyond the scope of the MULTI2HYCAT project and had not been carried out. Nevertheless, the two hybrid materials underwent $\mathrm{N}_{2}$ physisorption analyses and solid state MAS NMR spectra were recorded, to evaluate the effect of the passivation step on the textural properties of the samples and on the chemical speciation of $\mathrm{Si}$ and $\mathrm{C}$ atoms. $\mathrm{N}_{2}$ physisorption isotherms are reported in Fig. 3.23 A. All isotherms show a type IV behavior, which confirms the presence of mesopores.
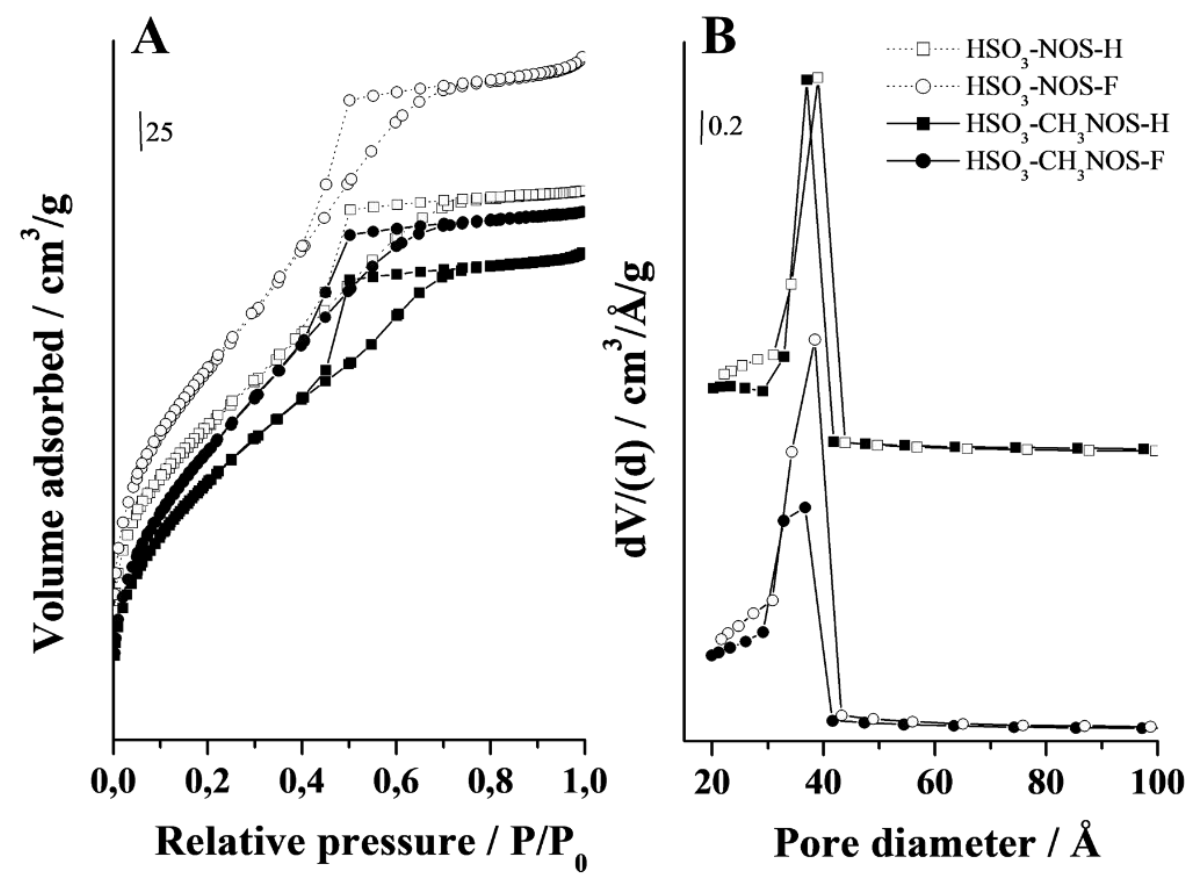

Figure 3.23 A: $\mathrm{N}_{2}$ adsorption/desorption isotherms at $77 \mathrm{~K}$ of hybrids $\mathrm{HSO}_{3}-\mathrm{NOS}^{-\mathrm{H}}, \mathrm{HSO}_{3}-$ NOS-F, $\mathrm{HSO}_{3}-\mathrm{CH}_{3} \mathrm{NOS}-\mathrm{H}$ and $\mathrm{HSO}_{3}-\mathrm{CH}_{3} \mathrm{NOS}-\mathrm{F}$. B: Pore-size distribution of the materials in the mesopore range. 
By comparing the analyses recorded on non-passivated samples with those recorded on passivated ones, it appears clear that the functionalization of the surface with HMDS reduces the free pore volume, as the overall $\mathrm{N}_{2}$ is lower for passivated hybrids. The BJH pore size distribution (Fig. 3.23 B) indicates a more prominent reduction of the area of the peak related to the mesoporous channels in the fluorinated sample $\left(\mathrm{HSO}_{3}-\mathrm{CH}_{3} \mathrm{NOS}\right.$ F), compared to the non-fluorinated one. The analysis of the isotherms with BET and $\mathrm{BJH}$ methods confirm this trends. The silanol capping process caused a reduction in the BET surface area of about $18 \%$ for $\mathrm{HSO}_{3}-\mathrm{CH}_{3} \mathrm{NOS}-\mathrm{F}$ (from 815 to $670 \mathrm{~m}^{2} / \mathrm{g}$ ) and of $14 \%$ for $\mathrm{HSO}_{3}-\mathrm{CH}_{3} \mathrm{NOS}-\mathrm{H}$ (from 680 to $590 \mathrm{~m}^{2} / \mathrm{g}$ ) Coherently, the $\mathrm{BJH}$ pore volume also dropped upon silanol capping. Specifically, $\mathrm{HSO}_{3}-\mathrm{CH}_{3} \mathrm{NOS}-\mathrm{F}$ experienced a $27 \%$ drop (from 0.52 to $0.38 \mathrm{~cm}^{3} / \mathrm{g}$ ), while $\mathrm{HSO}_{3}-\mathrm{CH}_{3} \mathrm{NOS}-\mathrm{H}$ experienced only a $17 \%$ drop (from 0.42 to $0.35 \mathrm{~cm}^{3} / \mathrm{g}$ ). More studies, focused on the portion of free volume accessible to the capping agent, would be needed to explain this trends.

After addressing the textural properties of the hydrophobic hybrids, solid state MASNMR spectra were recorded in order to address the effect of the silanol-capping step on the chemical speciation of $\mathrm{H}, \mathrm{C}$ and $\mathrm{Si}$ atoms. ${ }^{29} \mathrm{Si}$ spectra (Fig. 3.24) show the Q and T sites that had been observed in the spectra recorded on the non-passivated hybrid solids (reported in Fig. 3.13). Additionally, a signal centered at about $12 \mathrm{ppm}$ is observed and assigned to $\mathrm{M}$ silicon sites, i.e. silicon atoms bounded to three carbon atoms. ${ }^{96}$ Although this confirms the presence of trimethyl-silyl groups in the materials, the presence of $\mathrm{Q}^{3}$ sites indicates an incomplete silanol capping. Given the non-quantitative nature of the ${ }^{29} \mathrm{Si} \mathrm{CP} / \mathrm{MAS}$ NMR spectra, in which the magnetization is transferred to the silicon atoms from $\mathrm{H}$ atoms in their proximity, no coverage yield can be extrapolated. Moreover, considering that the hybrids were synthetized through one-pot processes, in which porosity is generated in a random fashion, it is likely that a fraction of the silanol groups are located in portions of the material inaccessible to HMDS moieties (e.g. ink-bottle cavities). Additionally, the use of silyl-derivatives of different molecular size used as cocondensation agents, further complicates the task of estimating the fraction of silanol groups available for the reaction with HMDS.

A possible way to study this aspect would be the adsorption of probe molecules having a steric hindrance similar to that of HMDS, and baring a functional group able to interact with surface silanols (e.g. amine). The adsorption would be followed by ${ }^{1} \mathrm{H}$ NMR spectroscopy. If the position of the signals (prior and after interaction with the probe) would allow deconvolution and integration, it might be possible to estimate what percentage of silanols is accessible, allowing in turn to calculate a coverage yield that would consider only the fraction of surface that plays a role in affecting mass transfer and adsorption of reactants during catalytic testing. 


\begin{tabular}{|c|c|c|c|c|}
\hline $\mathbf{T}^{2}$ & $\mathbf{T}^{3}$ & $\mathbf{Q}^{2}$ & $\mathbf{Q}^{3}$ & $\mathbf{Q}^{4}$ \\
\hline & OSi & $\mathrm{OH}$ & $\mathrm{OH}$ & OSi \\
\hline 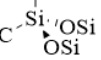 & $\mathrm{C}^{-\mathrm{Si} \cdot{ }^{\prime} \mathrm{OSi}}$ & $\mathrm{HO}^{-\mathrm{Si}_{1}^{\prime} \mathrm{OSi}}$ & $\mathrm{SiO}^{-\mathrm{Si}} \stackrel{\mathrm{O}}{\mathrm{OSSi}}$ & $\mathrm{SiO}^{-\mathrm{Si} \cdot \mathrm{COSi}^{\prime}}$ \\
\hline
\end{tabular}
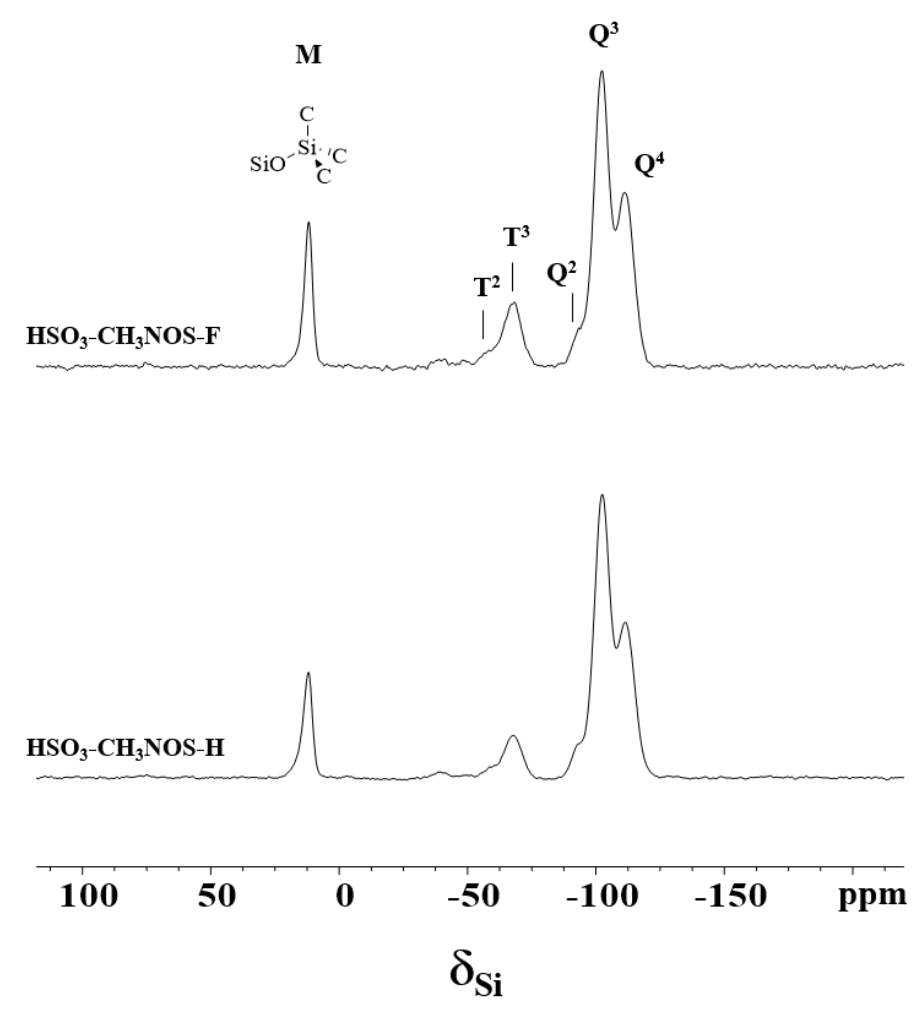

Figure 3.24: ${ }^{29} \mathrm{Si} \mathrm{CP/MAS} \mathrm{NMR} \mathrm{spectra} \mathrm{of} \mathrm{the} \mathrm{hydrophobic} \mathrm{hybrid} \mathrm{materials.}$

${ }^{1} \mathrm{H}$ MAS and spin-echo NMR spectra of the hybrids, previously outgassed for 90 minutes at $180^{\circ} \mathrm{C}$, were recorded (Fig. 3.25 A). Both spectra show an intense signal centered at $0 \mathrm{ppm}$, related to the methyl proton coming from the reacted HMDS. Interestingly, a signal centered at 1.7 related to the free silanol groups is also observed. This feature confirms the incomplete passivation with HMDS observed by ${ }^{29} \mathrm{Si}$ NMR.

${ }^{13} \mathrm{C}$ CP/MAS NMR spectra of the hydrophobic catalysts (Fig. $3.25 \mathrm{~B}$ ) show an intense signal centered at $0 \mathrm{ppm}$, due to the $\mathrm{C}$ atoms of the methyl groups with which the surface was passivated. The signals related to aliphatic carbons are present in the region between 0 and $60 \mathrm{ppm}$. Nevertheless, signals related to the aromatic carbon are barely visible, 
especially in sample $\mathrm{HSO}_{3}-\mathrm{CH}_{3} \mathrm{NOS}-\mathrm{F}$. This may be due to a lower degree of tethering, related to the presence of methyl groups on the surface and/or to a measurement limitation related to the long relaxation time that quaternary aromatic carbons need between each pulse to restore the magnetization. Additionally, each of the fluorine atom would Jcouple with its $\alpha$ carbon and with other carbon atoms of the aromatic ring, broadening the signal and making it more overlapped with the background.

It appears clear that capping of the surface silanol groups affected the hybrid catalysts in a negative fashion. However, this approach could still prove valid for preparing supported sulfonic acid catalysts for reactions involving hydrophobic substrates, such as the production of biodiesel.

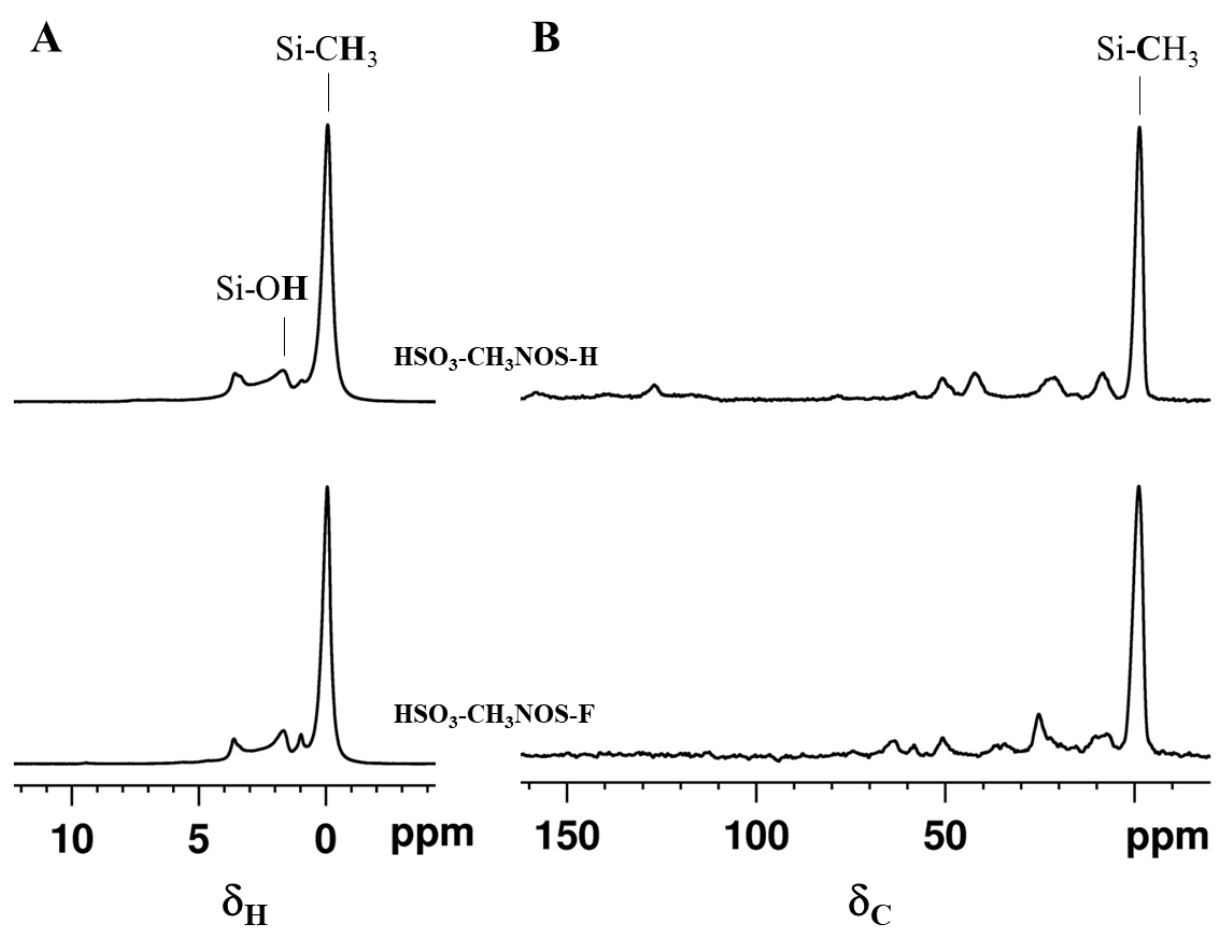

Figure 3.25: ${ }^{1} \mathrm{H}$ spin-echo NMR (A) and ${ }^{13} \mathrm{C}$ CPMAS NMR (B) spectra of the hydrophobic hybrid materials. 


\subsection{Conclusions}

The research reported in this Chapter, carried out within the frame of the MULTI2HYCAT European Project, aimed at synthesizing two silica-based hybrid catalysts baring sulfonic acid groups. One of them was designed to bare sulfonic acid groups attached to an aromatic ring and linked to the silica support by a urea group and a propyl linker. The other one was meant to bare sulfonic acid groups attached to a fluorinated aromatic ring and bounded to the silica support by an ether group and a propyl chain. The novelty of the study lies in the fact that multiple article reported enhanced catalytic activity whenever the sulfonic acid groups were either attached to a phenyl substituent or in close proximity to fluorine atoms. Nevertheless, the eventually beneficial effect of having the sulfonic acid attached to a fluorinated aromatic ring was not reported in the scientific literature so far.

- The two hybrid catalysts were successfully synthesized by using two slightly different approaches. For $\mathrm{HSO}_{3}-\mathrm{NOS}-\mathrm{H}$ sample, a silyl-derivative from sulfanilic acid was synthesized. The siloxane was co-condensed with tetramethyl ortosilicate (TMOS) in methanol/ $\mathrm{H}_{2} \mathrm{O}$, catalyzed by $\mathrm{F}^{-}$ions and finally the solid was exchanged in mildly acidic solution, to achieve the protonic form. In contrast, for $\mathrm{HSO}_{3}-\mathrm{NOS}$ F sample, 3-bromopropyl trimethoxysilane was co-condensed with TMOS in methanol $/ \mathrm{H}_{2} \mathrm{O}$, catalyzed by $\mathrm{F}^{-}$ions in a first step. Subsequently, a tethering reaction was carried out between the pendant Br-propyl moieties and sodium 2,3,5,6-tetrafluoro4-hydroxybenzenesulfonate. Lastly, the material was washed with $\mathrm{HCl} / \mathrm{ethanol}$ to achieve the protonic form. The successful synthesis of the two hybrid materials highlighted the versatility of silica-based strategies for synthesizing organic-inorganic solids, allowing to circumvent eventual limitations by slightly adapting the procedure. In this case, the synthesis of the silyl-derivative precursor for $\mathrm{HSO}_{3}-\mathrm{NOS}-\mathrm{F}$ synthesis was made impossible by the insolubility of sodium 2,3,5,6-tetrafluoro-4hydroxybenzenesulfonate in all solvents available for carrying out the reaction. By having one of the two functional groups involved in the reaction, already bounded to the solid support, allowed for the use of a high-boiling solvent (DMSO) that was able to dissolve sodium 2,3,5,6-tetrafluoro-4-hydroxybenzenesulfonate and was then removed by filtration at the end of the reaction.

- Multiple characterization techniques were implemented to study the hybrid materials. The textural properties of the hybrids were addressed by recording $\mathrm{N}_{2}$ physisorption and analyzing them using BET, BJH and NLDFT methods. The results were compared with a purely siliceous non-ordered silica. BET specific surface area values were consistent with mesoporous materials $\left(700-800 \mathrm{~m}^{2} / \mathrm{g}\right)$ as well as BJH pore volume values. Additionally, BJH analysis showed a pore size distribution ranging between 20 and $60 \AA$ with a peak centered at about $35 \AA$ A NDFT analysis allowed to detect a fraction of micropore contribution to the total porosity, in line with other studies involving this synthetic methodology. ${ }^{29} \mathrm{Si} \mathrm{CP} / \mathrm{MAS}$ NMR spectra of the hybrid catalysts showed the presence of Q sites (silicon atoms bounded to four oxygen 
atoms) and $\mathrm{T}$ sites (silicon atoms bounded to three oxygen atoms and one carbon atom), confirming the incorporation of the silyl-derivatives within the silica structure. From the point of view of the organic moieties, thermogravimetric analyses confirmed the stability of the hybrids at temperatures below $200-250{ }^{\circ} \mathrm{C}$. Moreover, the onset points of the first weight losses observed (not considering the one related to physisorbed water) were consistent with breakage of the chemical groups present in the linkers (urea group for $\mathrm{HSO}_{3}-\mathrm{NOS}-\mathrm{H}$ materials and ether group for $\mathrm{HSO}_{3}$ NOS-F material). FTIR spectroscopy allowed to observe signals related to the presence of key functional groups (e.g. sulfonic groups, urea groups, aromatic ring) and, together with ${ }^{13} \mathrm{C}$ solid state MAS NMR, confirmed the presence of the desired structures in both materials, beyond doubts. Moreover, it highlighted the presence of a fraction of unreacted Br-propyl moieties in sample $\mathrm{HSO}_{3}-\mathrm{NOS}-\mathrm{F}$, possibly located in regions of the material hardly accessible to substrate molecules. ${ }^{1} \mathrm{H}$ solid state MAS NMR gave insights on the proton speciation within the catalysts, showing signals consistent with aliphatic and aromatic protons from the organic moieties as well as the presence of silanols, both isolated and interacting with each other through $\mathrm{H}$ bond. A signal ascribed to the sulfonic acid proton was observed in $\mathrm{HSO}_{3}-\mathrm{NOS}-\mathrm{F}$ sample but not in $\mathrm{HSO}_{3}-\mathrm{NOS}-\mathrm{H}$ sample. This result was interpreted as the sulfonic acid group of hybrid $\mathrm{HSO}_{3}-\mathrm{NOS}-\mathrm{H}$ interacting through $\mathrm{H}$-bond with surface silanol groups, causing a shift and a broadening of the ${ }^{1} \mathrm{H}$ signal related to the sulfonic acid proton. This interpretation is in line with previously reported studies on polar functional groups bounded to silica surfaces by a propyl linker.

- In order to study the surface properties of the hybrids, probe molecules were adsorbed and their interaction was studied by spectroscopic techniques. To the knowledge of the candidate, this is the first time that these characterization techniques were implemented in the study of silica-base hybrid catalysts. Carbon monoxide adsorption was performed at $80 \mathrm{~K}$ and followed by FTIR. Interestingly, while this weakly basic probe molecule was able to interact with the mildly acidic silanols, it was not able to interact with the much more acidic sulfonic acid groups. In contrast, when ammonia adsorption was performed (followed by FTIR and ${ }^{1} \mathrm{H}$ solid state NMR), a clear signal related to the formation of ammonium ion was unambiguously detected. As the sulfonic acid groups interact through $\mathrm{H}$-bond with the nearby surface silanol, breaking this interaction would require the formation of a stronger one. The weakly basic character of carbon monoxide is not able to provide the system with such interaction (and associated energy gain) while much more basic ammonia molecules are able to form ion pairs with the sulfonic acid groups (which would become sulfonates), lowering the free energy of the system. Lastly, trimethylphosphine oxide (TMPO) was adsorbed onto the hybrids and studied by ${ }^{31} \mathrm{P}$ MAS NMR. The down-field shift of the signal of TMPO indicates that both hybrids have acidic properties comparable to those of pure $\mathrm{H}_{2} \mathrm{SO}_{4}$. 
- The hybrids were tested in the acetal formation reaction between benzaldehyde and ethylene glycol. Both catalysts showed remarkable activity and reusability, with catalyst $\mathrm{HSO}_{3}$-NOS-F showing a better preservation of the catalytic activity over multiple cycles, compared to hybrid $\mathrm{HSO}_{3}-\mathrm{NOS}-\mathrm{H}$. The latter, was also tested in other acetalization reactions, highlighting the capacity of these hybrid acid catalysts.

- A hydrophobic version of the organic-inorganic catalysts was prepared, by reacting surface silanols with hexamethyl disilazane as capping agent, through grafting methods. The resulting hybrid materials, $\mathrm{HSO}_{3}-\mathrm{CH}_{3} \mathrm{NOS}-\mathrm{H}$ and $\mathrm{HSO}_{3}-\mathrm{CH}_{3} \mathrm{NOS}-\mathrm{F}$, showed reduced specific surface area and pore volume, compared to non-passivated samples. ${ }^{29} \mathrm{Si},{ }^{13} \mathrm{C}$ and ${ }^{1} \mathrm{H}$ NMR confirmed the presence of trimethyl-silyl functionalities in the composition of the hybrid solids, although free silanol groups were also observed. Whether the remaining fraction of silanol groups was accessible to HMDS moieties remains unexplored. $\mathrm{HSO}_{3}-\mathrm{CH}_{3} \mathrm{NOS}-\mathrm{H}$ and $\mathrm{HSO}_{3}-\mathrm{CH}_{3} \mathrm{NOS}-\mathrm{F}$ samples showed good catalytic performances in the acetal formation reaction between benzaldehyde and ethylene glycol, although not as good as the hydrophilic hybrids. Tentative interpretations of these observations were made, but to explain these observations beyond doubt, more characterization would be required.

The procedures developed to synthesize $\mathrm{HSO}_{3}-\mathrm{NOS}-\mathrm{H}$ and $\mathrm{HSO}_{3}-\mathrm{NOS}-\mathrm{F}$ hybrid materials provide useful tools that can be implemented in the synthesis of multi-functional catalysts. An example, discussed within the MULTI2HYCAT consortium, is related with the synthesis of mesoporous organosilica-based hybrid materials baring aryl-sulfonic acid groups and functionalized with metallic species, such as Ru nanoparticles. The objective would be to prepare bi-functional hybrid catalysts featuring Brönsted acid sites and redox sites, for applications in the fine-chemistry or pharmaceutical industry. 


\subsection{References}

(1) Haskell, V. C.; Hammett, L. P. J. Am. Chem. Soc. 1949, 71, 1284-1288.

(2) Bernhard, S. A.; Hammett, L. P. J. Am. Chem. Soc. 1953, 75, 1798-1800.

(3) Yoshikawa, S.; Kim, O. K. Bull. Chem. Soc. Jpn. 1966, 39, 1515-1519.

(4) Wesley, R. B. J. Catal. 1974, 293, 288-293.

(5) Reinicker, R. A.; Gates, B. C. BAIChE J. 1974, 20, 933-940.

(6) Mo, X.; Lotero, E.; Lu, C.; Liu, Y.; Goodwin, J. G. Catal. Letters 2008, 123, 1-6.

(7) Tesser, R.; Casale, L.; Verde, D.; Di Serio, M.; Santacesaria, E. Chem. Eng. J. 2010, $157,539-550$.

(8) Özbay, N.; Oktar, N.; Tapan, N. A. Fuel 2008, 87, 1789-1798.

(9) Feng, Y.; He, B.; Cao, Y.; Li, J.; Liu, M.; Yan, F.; Liang, X. Bioresour. Technol. 2010, 101, 1518-1521.

(10) Liu, Y.; Wang, L. Chem. Eng. Process. Process Intensif. 2009, 48, 1152-1156.

(11) Guerreiro, L.; Castanheiro, J. E.; Fonseca, I. M.; Martin-Aranda, R. M.; Ramos, A. M.; Vital, J. Catal. Today 2006, 118, 166-171.

(12) Alba-Rubio, A. C.; Vila, F.; Alonso, D. M.; Ojeda, M.; Mariscal, R.; López Granados, M.. Appl. Catal. B Environ. 2010, 95, 279-287.

(13) Choudhary, H.; Nishimura, S.; Ebitani, K. Appl. Catal. A Gen. 2013, 458, 55-62.

(14) Inamuddin; Rangreez, T. A.; Asiri, A. M. Applications of Ion Exchange Materials in Chemical and Food Industries 2019, Springler.

(15) James, C. D; Gresham, W. F. Fluorocarbon vinyl ether polymer, 1963, US patent: US3282875A.

(16) Peterson A. H. Cationic ion exchange resin catalysts and process for their production and use, 1969, US patent: US3479322A.

(17) Sani, Y. M.; Daud, W. M. A. W.; Abdul Aziz, A. R. Appl. Catal. A Gen. 2014, 470, 140-161.

(18) Mimura, T. ( 12 ) United States Patent. 2002, 2 (12). Kaita, J.; Mimura, T.; Fukuoka, N.; Hattori, Y. Catalyst for transesterification, 2002, US patent: US6407269B2

(19) Melero, J. A.; Iglesias, J.; Morales, G. Green Chem. 2009, 11, 1285-1308.

(20) Qiao, Z.; Wang, Z.; Zhang, C.; Yuan, S.; Zhu, Y.; Wang, J. AIChE J. 2012, 59, 215 228.

(21) Sainio, T.; Laatikainen, M.; Paatero, E. Ion Exchange and Solvent Extraction. A series of advances, 2007, CRC press. 
(22) Pouilloux, Y.; Abro, S.; Vanhove, C.; Barrault, J. R J. Mol. Catal. A Chem. 1999, 149, 243-254.

(23) Abreu, F. R.; Alves, M. B.; Macêdo, C. C. S.; Zara, L. F.; Suarez, P. A. Z. J. Mol. Catal. A Chem. 2005, 227, 263-267.

(24) Chakrabarti, A.; Sharma, M. M. React. Polym. 1993, 20, 1-45.

(25) Santilli, D. S.; Gates, B. C. Handbook of Heterogeneous Catalysis, 2008, WileyVCH Verlag GmbH.

(26) Krawiec, P.; Kockrick, E.; Borchardt, L.; Geiger, D.; Corma, A.; Kaskel, S. J. Phys. Chem. C 2009, 7755-7761.

(27) Chang, B.; Fu, J.; Tian, Y.; Dong, X. RSC Adv. 2013, 3, 1987-1994.

(28) Xing, R.; Liu, Y.; Wang, Y.; Chen, L.; Wu, H.; Jiang, Y.; He, M.; Wu, P. Microporous Mesoporous Mater. 2007, 105, 41-48.

(29) Wang, X.; Liu, R.; Waje, M. M.; Chen, Z.; Yan, Y.; Bozhilov, K. N.; Feng, P. Chem. Mater. 2007, 19, 2395-2397.

(30) Sun, Z. P.; Zhang, X. G.; Tong, H.; Liang, Y. Y.; Li, H. L. J. Colloid Interface Sci. 2009, 337, 614-618.

(31) Wu, D.; Xu, F.; Sun, B.; Fu, R.; He, H.; Matyjaszewski, K. Chem. Rev. 2012, 112, 3959-4015.

(32) Wan, S.; Guo, J.; Kim, J.; Ihee, H.; Jiang, D. Angew. Chemie - Int. Ed. 2009, 48, 5439-5442.

(33) Zhang, D.; Hao, Y.; Zheng, L.; Ma, Y.; Feng, H.; Luo, H. J. Mater. Chem. A 2013, 1, 7584-7591.

(34) Choi, D. H.; Ryoo, R. J. Mater. Chem. 2010, 20, 5544-5550.

(35) Van Rhijn, W. M.; De Vos, D. E.; Sels, B. F.; Bossaert, W. D.; Jacobs, P. A. Chem. Commun. 1998, 3, 317-318.

(36) Lim, M. H.; Blanford, C. F.; Stein, A. Chem. Mater. 1998, 10, 467-470.

(37) Doustkhah, E.; Luque, R.; Kim, J.; Lin, J.; Ide, Y.; Yamauchi, Y.; Rostamnia, S.; Wu, K. C.-W.; Len, C.; Luo, X.; Bando, Y. Chem. - A Eur. J. 2018, 25, 1614-1635.

(38) Boveri, M.; Aguilar-Pliego, J.; Pérez-Pariente, J.; Sastre, E. Catal. Today 2005, 107, 868-873.

(39) Dacquin, J. P.; Cross, H. E.; Brown, D. R.; Düren, T.; Williams, J. J.; Lee, A. F.; Wilson, K. Green Chem. 2010, 12, 1383-1391.

(40) Pérez-Pariente, J.; Díaz, I.; Mohino, F.; Sastre, E. Appl. Catal. A Gen. 2003, 254, 173-188.

(41) Bossaert, W. D.; De Vos, D. E.; Van Rhijn, W. M.; Bullen, J.; Grobet, P. J.; Jacobs, 
P. A. J. Catal. 1999, 182, 156-164.

(42) Van Grieken, R.; Melero, J. A.; Morales, G. Appl. Catal. A Gen. 2005, 289, 143152.

(43) Zhao, W.; Salame, P.; Launay, F.; Gédéon, A.; Hao, Z. J. Porous Mater. 2008, 15, 139-143.

(44) Rostamnia, S.; Pourhassan, F. Chinese Chem. Lett. 2013, 24, 401-403.

(45) Srinivas, D.; Saikia, L. Catal. Surv. from Asia 2008, 12, 114-130.

(46) Gianotti, E.; Diaz, U.; Velty, A.; Corma, A. Catal. Sci. Technol. 2013, 3, 2677-2688.

(47) Zeidan, R. K.; Hwang, S. J.; Davis, M. E. Angew. Chemie - Int. Ed. 2006, 45, 63326335 .

(48) Zuo, D.; Lane, J.; Culy, D.; Schultz, M.; Pullar, A.; Waxman, M. Applied Catal. B, Environ. 2013, 129, 342-350.

(49) Parambadath, S.; Chidambaram, M.; Singh, A. P. Catal. Today 2004, 97, 233-240.

(50) Requies, J.; Güemez, M. B.; Arias, P. L. Applied Catal. B, Environ. 2014, 145, 34 42.

(51) Wang, X.; Cheng, S.; Chan, J. C. C.; Chao, J. C. H. Micropor. Mesopor. Mater. 2006, 96, 321-330.

(52) Habib, S.; Salam, P.; Launay, F.; Semmer-herledan, V.; Marie, O.; Zhao, W.; Antoine, G. J. Mol. Catal. A 2007, 271, 117-125.

(53) Harmer, M. A.; Sun, Q.; Michalczyk, M. J.; Yang, Z. Chem. Commun. 1997, 18031804.

(54) Alvaro, M.; Corma, A.; Das, D.; Fornés, V.; García, H. J. Catal. 2005, 231, 48-55.

(55) Macquarrie, D. J.; Tavener, S. J.; Harmer, M. A. Chem. Commun. 2005, 18, $2363-$ 2365.

(56) Rostami, A.; Tahmasbi, B.; Gholami, H.; Taymorian, H. Chinese Chem. Lett. 2013, 24, 211-214.

(57) Hajjami, M.; Ghorbani, F.; Bakhti, F. Appl. Catal. A Gen. 2014, 470, 303-310.

(58) Clapp, L. B. J. Chem. Educ. 1943, 20, 189-191.

(59) Pirez, C.; Lee, A. F.; Jones, C.; Wilson, K. Catal. Today 2014, 234, 167-173.

(60) Mbaraka, I. K.; Shanks, B. H. J. Catal. 2005, 229, 365-373.

(61) Karimi, B.; Zareyee, D. Org. Lett. 2008, 10, 3989-3992.

(62) Yang, Q.; Kapoor, M. P.; Inagaki, S. J. Am. Chem. Soc. 2002, 124, 9694-9695.

(63) Karaki, M.; Karout, A.; Toufaily, J.; Rataboul, F.; Essayem, N.; Lebeau, B. J. Catal. 
2013, 305, 204-216.

(64) Karam, A.; Alonso, J. C.; Gerganova, T. I.; Ferreira, P.; Bion, N.; Barrault, J.; Jérôme, F. Chem. Commun. 2009, 45, 7000-7002.

(65) Kapoor, M. P.; Yanagi, M.; Kasama, Y.; Yokoyama, T.; Inagaki, S.; Shimada, T.; Nanbu, H.; Juneja, L. R. J. Mater. Chem. 2006, 16, 3305-3311.

(66) Shylesh, S.; Wagener, A.; Seifert, A.; Ernst, S.; Thiel, W. R. Angew. Chemie - Int. Ed. 2010, 49, 184-187.

(67) Alauzun, J.; Mehdi, A.; Reyé, C.; Corriu, R. J. P. J. Am. Chem. Soc. 2006, 128, 87188719 .

(68) Deng, T.; Xu, G.; Fu, Y. Chinese J. Catal. 2020, 41, 404-414.

(69) Piscopo, C. G.; Maestri, G.; Paris, E.; Santacroce, V.; Maggi, R. Arkivoc 2015, 7, 19.

(70) Posternak, A. G.; Garlyauskayte, R. Y.; Yagupolskii, L. M. Tetrahedron Lett. 2009, $50,446-447$.

(71) Olah, G. A.; Laali, K.; Mehrotra, A. K. J. Org. Chem. 1983, 48, 3360-3362.

(72) Olah, G. A.; Arvanaghi, M.; Krishnamurthy, V. V. J. Org. Chem. 1983, 48, 33593360 .

(73) Armor, J. N. Appl. Catal. A Gen. 2001, 222, 407-426.

(74) Miletto, I.; Paul, G.; Chapman, S.; Gatti, G.; Marchese, L.; Raja, R.; Gianotti, E. Chem. - A Eur. J. 2017, 23, 9952-9961.

(75) Bordiga, S.; Ugliengo, P.; Damin, A.; Lamberti, C.; Spoto, G.; Zecchina, A.; Spanò, G.; Buzzoni, R.; Dalloro, L.; Rivetti, F. Top. Catal. 2001, 15, 43-52.

(76) Erigoni, A.; Newland, S. H.; Paul, G.; Marchese, L.; Raja, R.; Gianotti, E. ChemCatChem 2016, 8, 3161-3169.

(77) Erigoni, A.; Paul, G.; Meazza, M.; Hernández-Soto, M. C.; Miletto, I.; Rios, R.; Segarra, C.; Marchese, L.; Raja, R.; Rey, F.; Gianotti, E.; Díaz, U. Catal. Sci. Technol. 2019, 9, 6308-6317.

(78) Reale, E.; Leyva, A.; Corma, A.; Martínez, C.; García, H.; Rey, F. J. Mater. Chem. 2005, 15, 1742-1754.

(79) Paul, G.; Bisio, C.; Braschi, I.; Cossi, M.; Gatti, G.; Gianotti, E.; Marchese, L. Chem. Soc. Rev. 2018, 47, 5684-5739.

(80) Socrates, G. Infrared Characteristics Group Frequencies, 1980, Wiley.

(81) Paul, G.; Musso, G. E.; Bottinelli, E.; Cossi, M.; Marchese, L.; Berlier, G. ChemPhysChem 2017, 18, 839-849.

(82) Sharifi, M.; Wark, M.; Freude, D.; Haase, J. Microporous Mesoporous Mater. 2012, 
$156,80-89$.

(83) Bjørgen, M.; Joensen, F.; Spangsberg Holm, M.; Olsbye, U.; Lillerud, K. P.; Svelle, S. Appl. Catal. A Gen. 2008, 345, 43-50.

(84) Gianotti, E.; Dellarocca, V.; Marchese, L.; Martra, G.; Coluccia, S.; Maschmeyer, T. Phys. Chem. Chem. Phys. 2002, 4, 6109-6115.

(85) Zecchina, A.; Marchese, L.; Bordiga, S.; Pazè, C.; Gianotti, E. J. Phys. Chem. B 1997, 101, 10128-10135.

(86) Gianotti, E.; Dellarocca, V.; Marchese, L.; Martra, G.; Coluccia, S.; Maschmeyer, T. Phys. Chem. Chem. Phys. 2002, 4, 6109-6115.

(87) Kim, K. C.; Moschetta, E. G.; Jones, C. W.; Jang, S. S. J. Am. Chem. Soc. 2016, 138, 7664-7672.

(88) Pauling, L. The Hydrogen Bond. In The Nature of the Chemical Bond, 1960, Cornell University Press.

(89) Zheng, A.; Liu, S. Bin; Deng, F. Chem. Rev. 2017, 117, 12475-12531.

(90) Trickett, C. A.; Osborn Popp, T. M.; Su, J.; Yan, C.; Weisberg, J.; Huq, A.; Urban, P.; Jiang, J.; Kalmutzki, M. J.; Liu, Q.; Baek, J.; Head-Gordon, M. P.; Somorjai, G. A.; Reimer, J. A.; Yaghi, O. M. Nat. Chem. 2019, 11, 170-176.

(91) Zheng, A.; Li, S.; Liu, S. Bin; Deng, F. Acc. Chem. Res. 2016, 49, 655-663.

(92) Zheng, A.; Liu, S. Bin; Deng, F. Solid State Nucl. Magn. Reson. 2013, 55, 12-27.

(93) Liu, F.; Yi, X.; Chen, W.; Liu, Z.; Qi, C. Z.; Song, Y. F.; Zheng, A. Chem. Sci. 2019, $10,5875-5883$

(94) Wuts, P.; W. Greene, T. Protective Groups In Organic Synthesis, 2006, WileyInterscience, Ed.

(95) Firouzabadi, H.; Iranpoor, N.; Karimi, B. Synlett 1999, 1999, 321-323.

(96) Emery, L.; Buzare, J.; Derouet, D.; Forgeard, S.; Brosse, J. J. Polym. Sci. Part A Polym. Chem. 1997, 437-453. 



\section{Chapter 4}

\section{Hybrid Mesoporous Silica-Supported Base Organocatalysts for C-C Bond Formation}

Hybrid mesoporous organocatalysts have been prepared by grafting commercial and custom-made silyl-derivatives onto MCM-41 supports. The hybrid catalysts were characterized and tested for different reactions involving $C-C$ bond formation, such as Knoevenagel condensations and Michael addition. Reaction mechanisms have been proposed for each reaction and the beneficial effect of the surface silanol groups on the catalytic activity was demonstrated. Finally, the catalysts were tested in the condensation between furfural and methyl isobutyl ketone and the most performing catalyst was selected for the synthesis of a multi-functional catalyst.

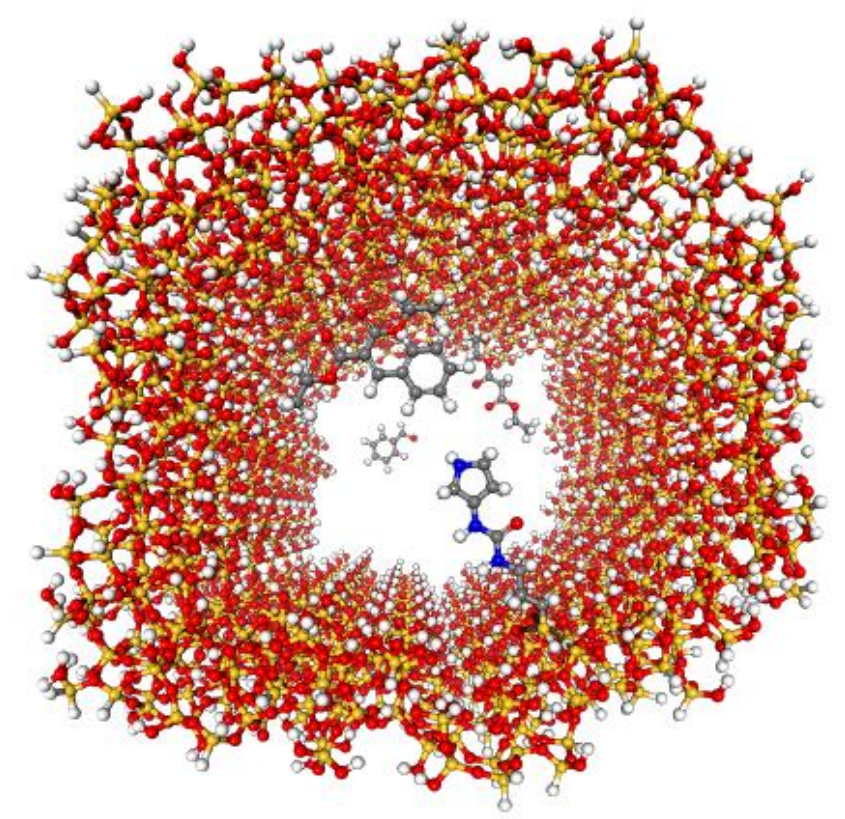



A base catalyzed reaction is defined as one that is accelerated by the interaction between an electron-pair donor (catalyst) and a substrate. More specifically, if the catalyst acts by donating an electron pair to an empty orbital of a substrate (acceptor), we talk about Lewis base catalysis. On the other hand, if the interaction occurring is a proton transfer from the substrate to the catalyst, we talk about Brönsted base catalysis. The electron pair can be donated on different orbitals of the acceptor. Consequentially, transfers from non-bonding electron pairs to antibonding orbitals with $\pi$ character $\left(\mathrm{n} \rightarrow \pi^{*}\right)$, to antibonding orbitals with $\sigma$ character $\left(n \rightarrow \sigma^{*}\right)$ and to non-bonding $n^{*}$ orbitals $\left(n \rightarrow n^{*}\right)$ can occur. ${ }^{1}$

As for acid catalyzed reactions, the use of mineral bases such as $\mathrm{NaOH}$ and $\mathrm{KOH}$ presents several drawbacks such as neutralization and disposal of the generated salt, as well as the impossibility of recycling and reusing the catalysts. Purely inorganic metal oxides such as $\mathrm{BaO},{ }^{2} \mathrm{SrO},{ }^{3} \mathrm{CaO},{ }^{4} \mathrm{MgO}^{5,6}$ and others ${ }^{7}$ were the first class of materials implemented as heterogeneous catalysts. Oxygen atoms featuring coordinative vacancies, i.e. those located on edges and wedges of the crystals, are able to donate an electron pair, therefore acting as a base. Consequentially, efforts have been made to increase the number of defects in these materials, ${ }^{8}$ which proved to be beneficial for the catalytic activity.

In the last few decades, the development of hybrid organic-inorganic catalysts introduced the possibility of preparing organocatalysts featuring base functional groups that could be supported onto inorganic solid frameworks and be separated easily from the reaction mixture and reused. The variety of organic base catalysts available makes it possible to appropriately choose a specific active site, based on activity and selectivity toward the desired product. This concept was described in Chapter 1, where studies involving different amines were reported, showing some supported amines being optimal catalysts for some reactions and other amines being optimal for other reactions. Clearly, the activity of supported base organocatalysts cannot be rationalized only upon their basic strength, in terms of $\mathrm{pKa}$, but other aspects such as nucleophilicity and the reaction mechanisms can play a key role. ${ }^{9-11}$

Silica-based materials functionalized with amine groups are known to efficiently catalyze $\mathrm{C}-\mathrm{C}$ coupling reactions, especially when weak acid sites are present in close proximity to amine function. Cooperative effect of multiple active sites is also well known. Undoubtedly, the most notable example are enzymes, in which multiple active sites (e.g. acid, base, H-bond donor) co-catalyze a given reaction. The complex enzyme architecture allows the catalytic sites and substrates to adopt a favorable conformation to promote the cooperative interaction, as functional groups placement and overall flexibility of the structure are optimal for such synergy to occur. Slight modifications of these parameters can have a major impact on mechanistic pathways and catalytic rates. ${ }^{12}$

Accounting for all variables contributing to this synergy and properly understanding the cooperative action occurring between the two sites are crucial aspects in the rational design of an optimal acid-base catalyst. ${ }^{13}$ Moreover, such insights could improve the rational design of hybrid catalysts featuring additional functional groups, such as metal nanoparticles or clusters. Many studies have been conducted by several authors, focused 
on understanding the effect of the strength of the base groups, the strength of the acid group and the distance between the two sites and others. The scope of this brief introduction will be to discuss some relevant studies involving the optimal design of such catalysts for aldol and nitroaldol condensations, which will pave the way to the experimental work discussed in this Chapter. 


\subsection{Amine-silanol cooperative catalysis for $\mathrm{C}-\mathrm{C}$ bond formation}

Lauwaert et al. prepared a series of amines supported onto commercial mesoporous silica, in which the surface silanols acted as the weak acid sites. In addition, they prepared the passivated counterpart of such catalysts by capping the surface silanol groups with trimethyl-silyl groups (Fig $4.1 \mathrm{~A}$ ). ${ }^{14} \mathrm{~A}$ kinetic model of the acid-base catalyzed aldol condensation was proposed (Fig. 4.1 B). Reactions both in presence and in absence of surface silanols were considered.
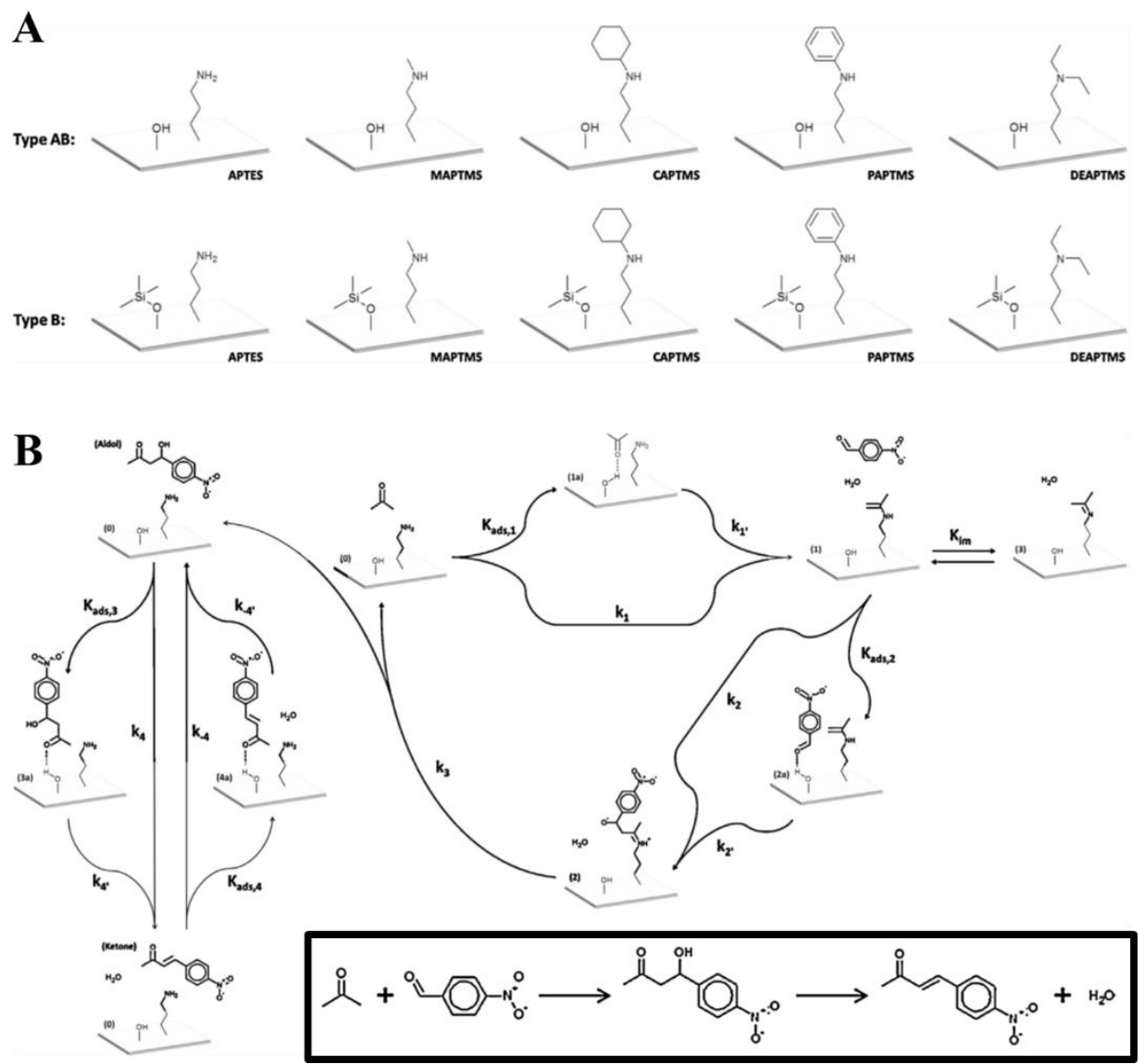

Figure 4.1 A: Schematic representation of the hybrid catalysts. B: Proposed reaction mechanisms for the acid-base catalyzed 4-nitrobenzaldehyde with acetone (reported in the inset). Adapted from ref. 14. 
When there are no promoting silanol groups available, the first step is the formation of an enamine (1) through a nucleophilic addition of acetone to the amine active site. The enamine is in equilibrium with the corresponding unreactive imine (3) which slows down the overall reaction rate. For the aldol condensation to occur, 4-nitrobenzaldehyde reacts with the enamine yielding an iminium ion (2). Water-assisted desorption of the iminium ion leads to the aldol product. Finally, the ketone is formed by dehydration of the aldol product. The presence of silanol groups facilitates the nucleophilic reaction by activation of the carbonyl bond of the substrate through hydrogen-bridge interactions (1a, 2a, 3a and $4 \mathrm{a}$ in Fig. $4.1 \mathrm{~B}$ ). The authors reported an increase in the selectivity toward the unsaturated product upon increase in temperature. Previous studies suggested that a decrease in the reaction rate can occur, due to the reaction between the silica-supported primary amine and nitro-benzaldehyde leading to the formation of a stable imine. ${ }^{15} \mathrm{Lau}-$ waert et al. reported that this effect was limited by using an excess of acetone in the reaction mixture. Moreover, the authors conclusively reported a higher TOF in the presence of surface silanols, regardless of the structure of the grafted amine, which confirms the beneficial effect of the established synergy between acid and base sites. Regarding the amine structure, when the catalyst was a primary amine, the carbinolamine could dehydrate and form an imine (inhibiting species). On the other hand, when a secondary amine was used as a catalyst, the formation of an imine was prevented due to the lack of a second hydrogen on the nitrogen, which resulted in a higher enamine concentration compared to when the active site is a primary amine. Consequentially, a higher reaction rate was observed. When the catalyst was a tertiary amine, no carbinolamine intermediate could be formed, due to the lack of hydrogen atoms bounded to the nitrogen, with consequentially lower catalytic activity than that of primary and secondary amines. ${ }^{14} \mathrm{It}$ should be highlighted that although in the context of this type of hybrids surface silanols are often referred to as "acid sites", they actually behave like H-bond partners. ${ }^{16}$

The innately acidic nature of the surface of silica support make them the ideal support for the design of catalysts that are require to act under cooperative regime. Nevertheless, studies have been carried out to understand the effect of using supported carboxylic acids as acid sites, together with amines. Brunelli et al. prepared a series of materials containing low loadings $(0.36 \mathrm{mmol} / \mathrm{g})$ of primary amine, to ensure the presence of enough silanol groups to act cooperatively with the base sites. The materials were prepared by grafting the silyl-derivatives onto the surface of the silica in a sequential fashion, firstly reacting the 3-aminopropyl triethoxysilane and then the protected carboxylic acid organosilane derivative. A fraction of the amino-functionalized silica was not functionalized with carboxylic acid groups and was used for comparison. By following the procedure, the authors ensured to have the same amount of amine grafted onto the catalysts. The catalysts were tested in the reaction between 4-nitrobenzaldehyde and acetone. The amine-silanol cooperative catalyst (Fig. 4.2, cat. 1) showed an initial TOF of $3.0 \mathrm{~h}^{-1}$, which the authors reported as high catalytic activity for primary amines, confirming the efficient acid-base synergy. 


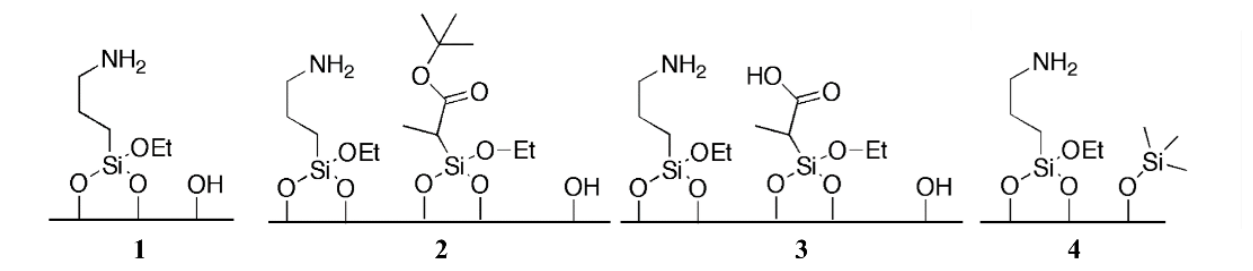

Figure 4.2: Hybrid bi-functional catalysts tested in the reaction of 4-nitrobenzaldehyde with acetone. Adapted from ref. 17.

By introducing the protected carboxylic acid, the catalytic activity dropped, compared to the amine-silanol material, with an initial TOF of $1.5 \mathrm{~h}^{-1}$ (Fig 4.2, cat. 2). Finally, unprotecting the carboxylic acid provided an amine-carboxylic acid organosiliceous material which showed the lowest catalytic activity of the three catalysts with an initial TOF of $1.2 \mathrm{~h}^{-1}$ (Fig. 4.2, cat. 3). Surface silanols proved to be better cooperative partners than carboxylic acids. Such trend was ascribed to differences in $\mathrm{pKa}$ values for silanols and carboxylic acids, with the former being optimal for cooperative catalysis. HMDS-capping of surface silanols induced a drop of the initial TOF to $0.8 \mathrm{~h}^{-1}$ (Fig. 4.2, cat. 4). ${ }^{17}$

Kinetics studies confirm the importance of such synergy between the amine group and the surface silanols. Collier et al. studied the kinetics of cooperatively catalyzed aldol and nitroaldol condensations, using butylamine-functionalized MCM-41 catalysts. Preexponential factors and activation energies were calculated by Arrhenius analysis of two catalysts with similar amine loadings. One of them featured silanol groups able to cooperatively interact with the amine sites (acid-base catalysis), while the other was treated with a silanol-capping reagent to prevent such cooperativity. The catalytic activity was observed to decrease for both aldol and nitroaldol condensations when the surface was functionalized with trimethylsilyl moieties and higher temperatures were required to accurately measure reactions rates, which emphasizes the crucial role played by the weakly acidic silanols in the catalytic cycles. Additionally, the authors found the loss of acid sites to be more detrimental to the catalytic activity for the aldol condensation reactions compared to the nitroaldol condensation. This was evidenced by the more prominent decrease in the pre-exponential factor for the aldol condensation when silanols were not available for synergistic action. Silanol groups act by activating acetone molecules through $\mathrm{H}$-bond with the oxygen atom. Consequentially, whenever surface silanols were passivated with trimethylsilyl groups, the reaction rate slowed down, which translated in a decrease in the pre-exponential factor of the Arrhenius equation. ${ }^{18}$

Molecular dynamics (MD) simulations also point out the key role played by surface silanols. Kim et al. carried out MD to study the cooperatively catalyzed aldol condensation between 4-nitrobenzaldehyde and acetone by alkylamine (or alkylenamine)-grafted sil- 
ica surfaces, with specific focus on the mechanism of the catalytic activation of 4-nitrobenzaldehyde and acetone by the acidic surface silanols and subsequent nucleophilic attack of the basic amine functional group at the activated reactant. They concluded that two main factors affect the synergy between acid and base sites, namely the competition between amine and silanol sites to form a hydrogen bond with a reactant and the flexibility of the alkylamine backbone. Increasing the flexibility of the alkylamine facilitates the nucleophilic attack of the amine on the reactants. Specifically, these simulations suggested $\mathrm{C} 3$ and $\mathrm{C} 4$ alkylamines to have the highest probability of reacting. ${ }^{19}$

These findings are in line with experimental studies indicating a correlation between the length of the alkylamines chains and their ability to catalyze aldol condensations. Brunelli et al. studied the effect of the length of such linker on the performances of the hybrid porous organosiliceous materials. They prepared an array of silica-supported amines, bounded to the surface through linkers of different size (Fig. 4.3 A). Silanol-capped versions of the catalysts were also prepared. Firstly, the materials were tested for $\mathrm{CO}_{2}$ capture (Fig. $4.3 \mathrm{~B}$ ), showing a much lower adsorption capacity for linkers shorter than C3. Additionally, no further increase in the adsorption was observed for materials featuring butyl or pentyl linkers. For HMDS-capped materials, the $\mathrm{CO}_{2}$ adsorption capacity was significantly lower for solids featuring $\mathrm{C} 3$ or longer linkers, as the capping molecule blocked the cooperative interaction needed for efficient $\mathrm{CO}_{2}$ adsorption. Non-passivated and passivated hybrids were also tested for their catalytic activity in the aldol condensation of 4-nitrobenzaldehyde with acetone (Fig. 4.3 C, D and E). Upon increasing the length of the carbon chain, up to $\mathrm{C} 3$, a surge in the reaction rate was observed. Further increase in the linker length beyond propyl resulted in identical catalytic rates. Lastly, as for $\mathrm{CO}_{2}$ adsorption, silanol-capping proved to be detrimental for the performances of the hybrids, once again confirming the beneficial cooperative effect of base sites and surface silanols. $^{12}$

Based on the studies described so far, it appears that the effect of the alkyl linker length is important because it affects the distance between surface silanols and amine groups, ultimately influencing the simultaneous interaction of the two sites can have with the substrate molecules. Another factor that can influence the distance between the two active sites is the curvature of the surface, which is strictly related to the pore size. 


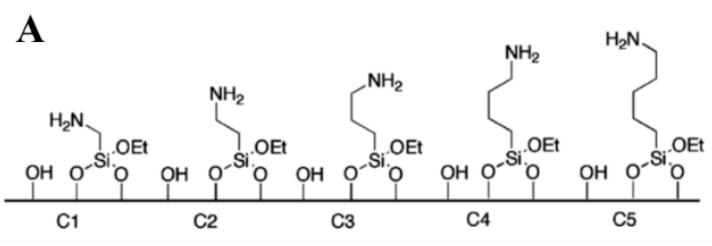

C
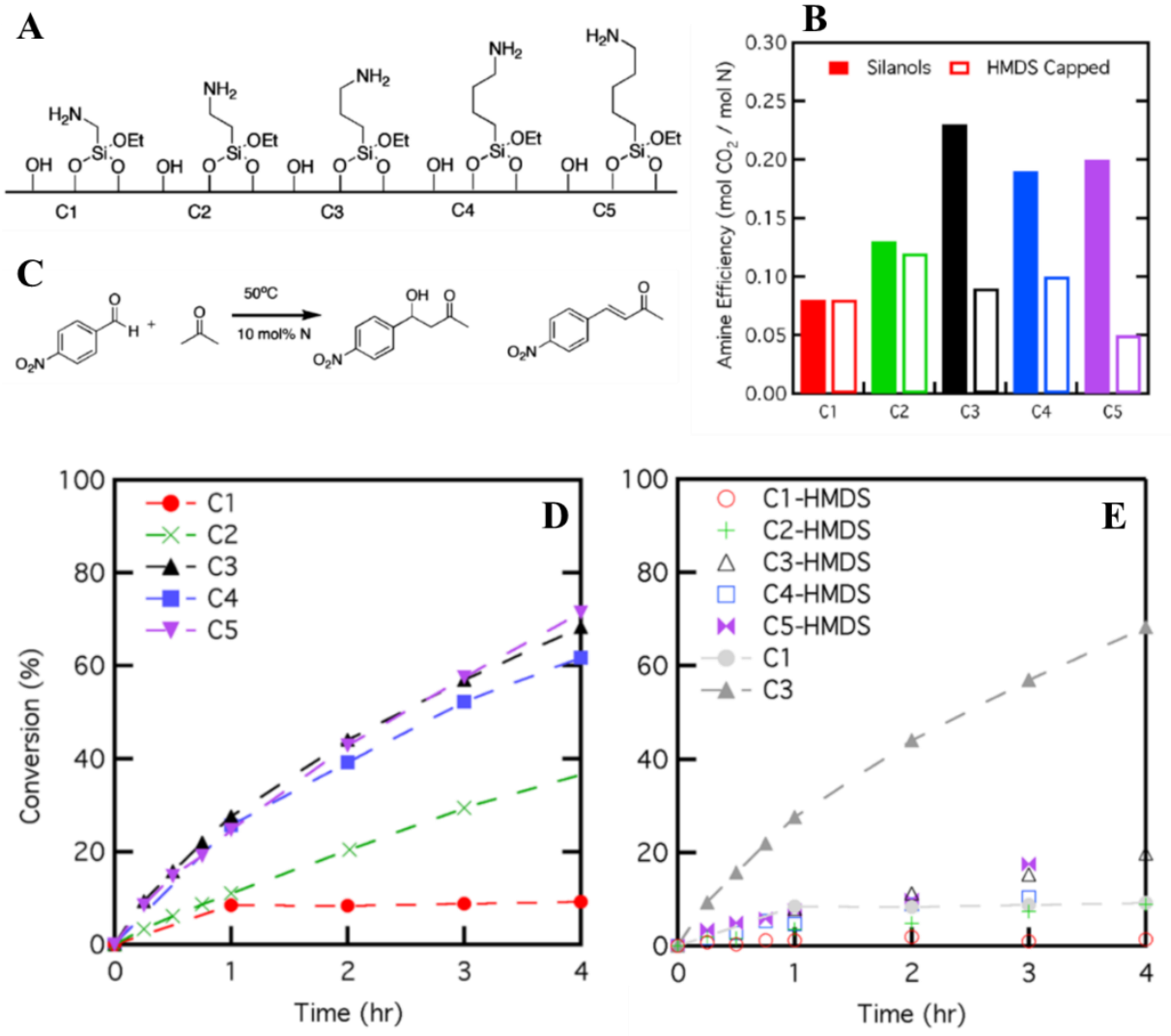

Figure 4.3 A: Silica-supported alkylamines used as adsorbent and as heterogeneous catalysts. B: Amine efficiencies for $\mathrm{CO}_{2}$ adsorption $(400 \mathrm{ppm}$ in $\mathrm{He})$. C: Schematic representation of the aldol condensation between 4-nitrobenzaldehyde and acetone. Performances of the non-passivated (D) and passivated $(E)$ hybrids in the reaction between of 4-nitrobenzaldehyde and acetone. Adapted from ref. 12.

The materials utilized in the previous study ${ }^{12}$ were developed using SBA- 15 as support (pore diameter of $6.5 \mathrm{~nm}$ ). Considering the average C-C bond length of $0.154 \mathrm{~nm}$, the aminosilanes can be expected to extend from the surface approximately $0.5 \mathrm{~nm}$ (Fig. 4.4). Such a difference in the length scales between the extension of the aminopropyl moieties and pore size means that from the perspective of the organic residues, the surface appears relatively flat. However, upon changing the pore diameter significantly, a change in amine-silanol cooperativity should be expected. To test the effect of the pore size on the amine-silanol cooperativity, Brunelli and co-workers prepared a series of MCM-41-supported aminoalkylsilanes, analogous to the series of catalysts reported in 
Fig. 4.3 A, described above. The two series of hybrids were compared in the condensation between nitrobenzaldehyde and acetone. Firstly, it can be observed that the catalytic activity of the MCM-C3 hybrid (smaller pore diameter) was identical to that of SBA-C3 (larger pore diameter), with an initial TOF of $3.0 \mathrm{~h}^{-1}$ (Fig. $4.4 \mathrm{~B}$ ). Notably, the MCM-C2 catalyst showed a TOF equal to the MCM-C3 sample and much higher activity than its SBA-supported counterpart. Changing the curvature of the pores brought the aminosilane closer to the silanol groups on the surface and therefore a shorter linker was flexible enough to allow amine-silanol cooperativity. Surprisingly, longer linker size (from C4 up) caused a drop in the catalytic activity of the MCM-supported catalysts. It appears that the presence of long linkers in the small pores strongly perturbs the cooperative system, although the reasons for this behavior are currently unclear. These results demonstrate that the curvature of the surface is a key factor to be kept into account for optimizing the synthesis of hybrid catalysts able to perform cooperative amine-silanols catalysis. Moreover, the study clearly empathizes the interdependency of two variables, namely pore size and linker length, on the distance between the amine group and the silanol groups present on the surface. ${ }^{16}$

\section{A}

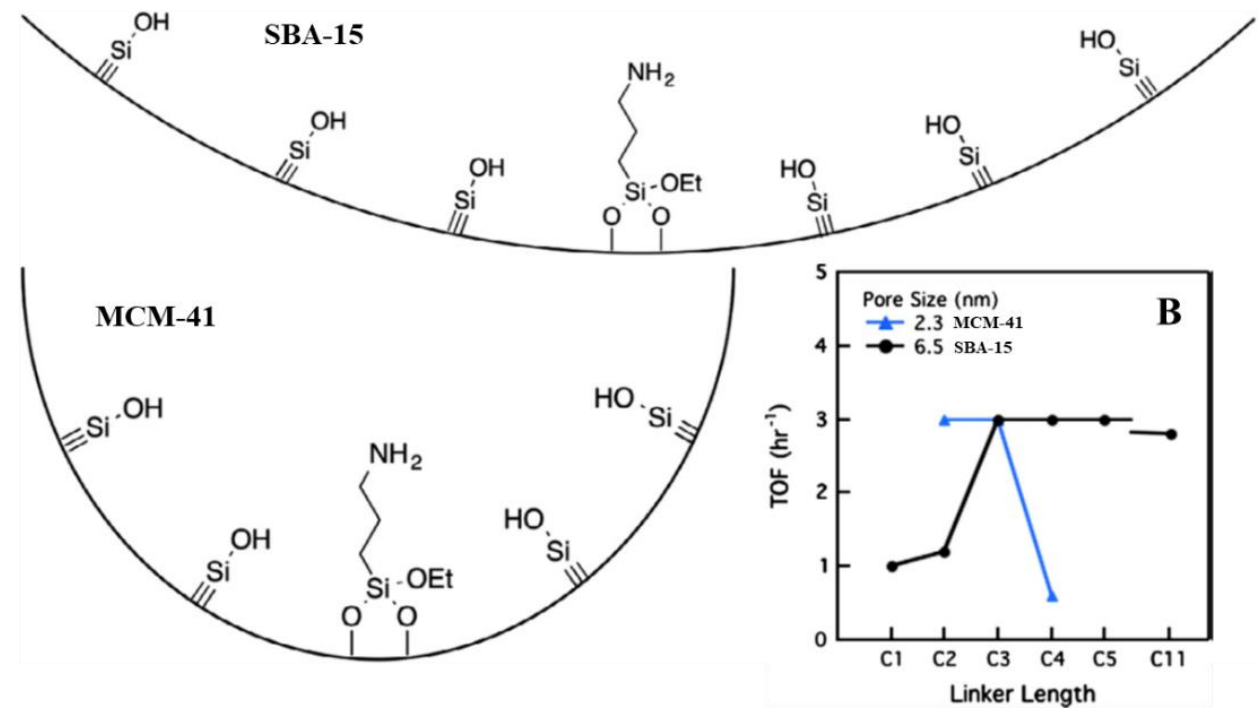

Figure 4.4 A: Schematic representation of the curvature effects on controlling the distance between amine groups and surface silanols. B: Initial turnover frequency of 4-nitrobenzaldehyde in the aldol condensation with acetone at $50{ }^{\circ} \mathrm{C}$. Adapted from ref. 16. 


\subsection{Scope of this work}

The research activity described in this Chapter is developed upon the needs of the industrial partners of the MULTI2HYCAT project. ${ }^{20}$ The final goal will be to prepare a catalyst featuring a base site able to catalyze an aldol condensation and a metal site, able to perform hydrogenation reactions (Fig. 4.5).

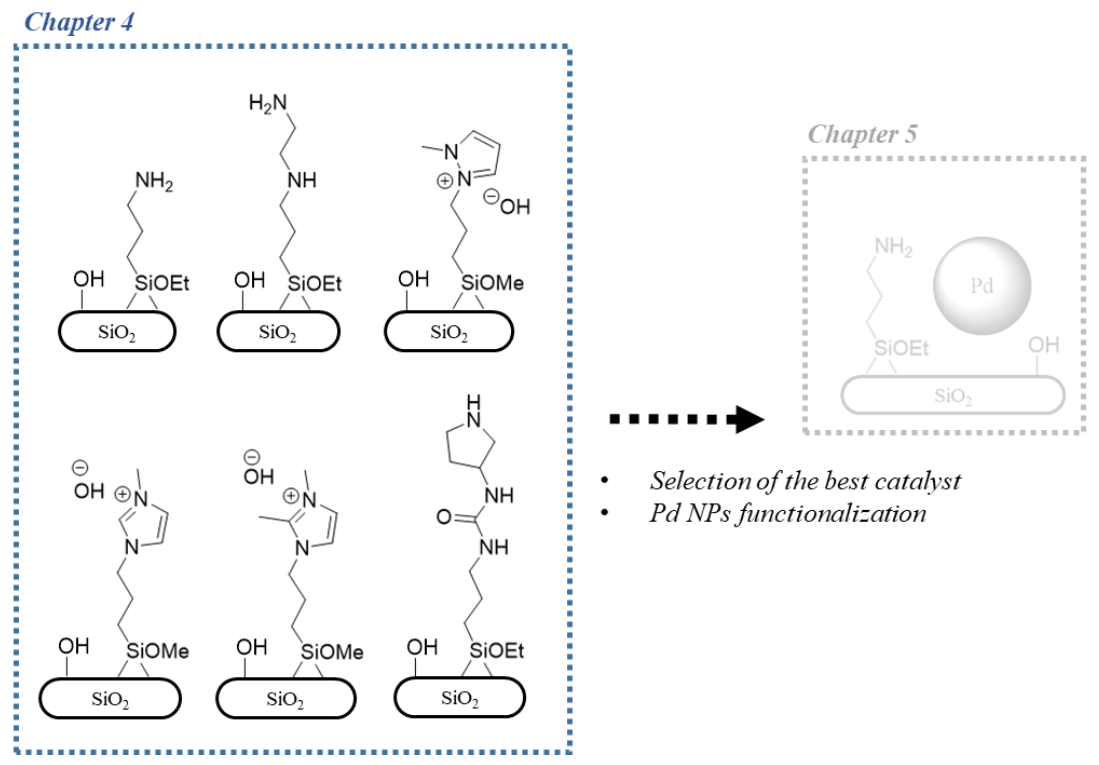

Figure 4.5: Roadmap of the synthesis of the multi-functional hybrid catalyst.

- The first step in the design of the multi-site catalyst will be to select an array of possible base sites and to support them onto mesoporous silica. The selection of organic moieties is going to include amino, diamino and pyrrolidine molecules, pyrazolium and imidazolium hydroxides functionalities. A total of six silyl-derivatives will be either bought from commercial sources or custom made in the lab and will be grafted onto MCM-41 supports. Whenever possible, a C3 linker is going to be used. As described in the introduction of this Chapter, linkers of such length are reported to be optimal for promoting amine-silanol cooperative catalysis, especially when the organic moieties are grafted within pore channels of about $2.3 \mathrm{~nm}$, such as those of the MCM-41. A passivated version of the catalyst carrying primary amines as active sites will be prepared, by reacting surface silanols with HMDS. The resulting hybrid will be characterized and tested, along with the non-passivated catalysts. 
- The resulting hybrids catalysts are going to be thoroughly characterized, to access their properties. X-ray powder diffraction, $\mathrm{N}_{2}$ physisorption anaylsis, thermogravimetric anaylsis, FTIR and ${ }^{13} \mathrm{C}$ and ${ }^{29} \mathrm{Si}$ NMR spectroscopies will be carried out to study structural, textural, thermal and surface properties of the hybrid materials.

- The catalysts will be tested in the Knoevenagel condensation between benzaldehyde and several substrates with different $\mathrm{pKa}$ values (featuring different activated methylenic groups), such as ethyl cyanoacetate $(\mathrm{pKa} \approx 9)$, ethyl acetoacetate $(\mathrm{pKa} \approx 11)$ and diethyl malonate $(\mathrm{pKa} \approx 13)$. A reaction mechanism will be proposed.

- The hybrid organosilica materials will also be studied as solid catalysts in the Michael addition of ethyl 2-oxocyclopentane 2-carboxylate and methyl vinyl ketone. A reaction mechanism will be proposed.

- Finally, the catalysts will be tested in the aldol condensation of furfural and methyl isobutyl ketone (MIBK), which constitutes the first step of the cascade reaction of interest for the MULTI2HYCAT consortium. The best catalyst for this reaction will be selected for further functionalization with Pd nanoparticles and will be described in Chapter 5.

- The last Section of this Chapter the synthesis of silica-supported phosphazenium oxide (P1) moieties will be described. These catalysts have been previously supported onto solid supports in a few instances, although through procedures involving harmful reactants and harsh conditions. After describing the state of the art of heterogeneous phosphazenes, a more versatile and easy synthetic procedure will be proposed. 


\subsection{Experimental}

\subsubsection{Synthesis of the materials}

Compounds are named according to the nomenclature reported in Fig. 4.6.

Synthesis of the MCM-41 support: $13.66 \mathrm{~g}$ of cetyltrimethylammonium bromide (7.6 mmol) were dissolved in $91 \mathrm{~mL}$ of distilled water together with $23.66 \mathrm{~g}$ of tetramethylammonium hydroxide solution $\left(25 \mathrm{wt} \%\right.$ in water) in a $250 \mathrm{ml}$ flask at $40{ }^{\circ} \mathrm{C}$. The mixture was vigorously stirred until complete dissolution of the surfactant. $15 \mathrm{~g}$ of Aerosil 200 were added and the resulting slurry was stirred for $1 \mathrm{~h}$, at room temperature. The gel was then transferred into a stainless steel autoclave and reacted for $24 \mathrm{~h}$ under static conditions at $135{ }^{\circ} \mathrm{C}$. The solid obtained was filtered and washed abundantly with distilled water and finally dried at $100{ }^{\circ} \mathrm{C}$ overnight. The solid was then calcined heating at $3{ }^{\circ} \mathrm{C} /$ min under a flow of $\mathrm{N}_{2}$ until $540{ }^{\circ} \mathrm{C}$, temperature at which the gas flow was switched to air. The temperature was kept for $6 \mathrm{~h}$ before allowing to cool down.

Synthesis of silyl-derivative III: $200 \mathrm{mg}$ of 2-aminopyrrolidine $(2.32 \mathrm{mmol})$ and $564 \mathrm{mg}$ of 3-isocyanatopropy ltriethoxysilane $(2.32 \mathrm{mmol})$ were mixed in a $\mathrm{N}_{2}$ filled Schlenck containing dry $\mathrm{CH}_{3} \mathrm{CN}$, at $-57^{\circ} \mathrm{C}$. The mixture was stirred at $-57^{\circ} \mathrm{C}$ for $6 \mathrm{~h}$. Afterwards, the solvent was removed under reduced pressure and the resulting gel was washed with diethyl ether.

Synthesis of silyl-derivative IV-VI: $387 \mathrm{mg}$ of 3-iodopropyltrimethoxysilane $(1.3 \mathrm{mmol})$ and the equivalent moles of the azole compound (110 mg for 1-methylpyrazole and 1methylimidazole and $128 \mathrm{mg}$ for 1,2-dimethylimidazole) were mixed in dry $\mathrm{CH}_{3} \mathrm{CN}$ under $\mathrm{N}_{2}$. The resulting solution was refluxed overnight. The solvent was then removed under reduced pressure and the gel was washed with diethyl ether.

Grafting of silyl-derivatives I-III on MCM-41: $2 \mathrm{~g}$ of MCM-41 were placed in a Schlenck and outgassed at $150{ }^{\circ} \mathrm{C}$ for $2 \mathrm{~h}$. Dry toluene was added and the mixture was stirred at rT for a few minutes. After that, the silyl base precursor was added to the mixture. The amount added was $4 \% \mathrm{~mol}$ of the total amount of silica, $1.3 \mathrm{mmol}$, corresponding to 295 $\mathrm{mg}$ for aminopropyl triethoxysilane (I), $297 \mathrm{mg}$ for $\mathrm{N}$-(2-aminoethyl)-3-(trimethoxysilyl)propylamine (II) and $445 \mathrm{mg}$ for silyl-derivative III. The resulting mixture was reacted at $80^{\circ} \mathrm{C}$ for $24 \mathrm{~h}$. After this time, the mixture was filtrated and washed with $\mathrm{CH}_{2} \mathrm{Cl}_{2}$ and ethanol. The materials were finally dried overnight at $100{ }^{\circ} \mathrm{C}$.

Grafting of silyl-derivatives IV-VI on MCM-41: $2 \mathrm{~g}$ of MCM-41 were placed in a Schlenck and outgassed at $150{ }^{\circ} \mathrm{C}$ for $2 \mathrm{~h}$. Dry $\mathrm{CH}_{3} \mathrm{CN}$ was added and the mixture was stirred at $\mathrm{rT}$ for a few minutes. After that, the silylated base precursor was added to the mixture and stirred for $1 \mathrm{~h}$ at room temperature. The amount added was $4 \% \mathrm{~mol}$ of the total amount of silica, $1.3 \mathrm{mmol}$, corresponding to $496 \mathrm{mg}$ for IV and V and $515 \mathrm{mg}$ for VI. After that, 1 eq of triethylamine $(186 \mu \mathrm{L})$ was added and the mixture was refluxed for $24 \mathrm{~h}$. The mixture was then filtrated and washed with $\mathrm{CH}_{3} \mathrm{CN}$ and acetone and the resulting solid was stirred three times in $0.2 \mathrm{M}$ solution of TMAOH in methanol for 30 
$\mathrm{min}$, at room temperature, in order to exchange the halogen for hydroxide. The final solid was washed extensively with distilled $\mathrm{H}_{2} \mathrm{O}$ and acetone and dried overnight at $100{ }^{\circ} \mathrm{C}$.

Syntheis of 1-HMDS: $0.5 \mathrm{~g}$ of MCM-41 functionalized with aminopropyl groups (catalyst 1) were outgassed at $150{ }^{\circ} \mathrm{C}$ for $2 \mathrm{~h}$. A solution of hexamethyldisilazane (HMDS, $1.25 \mathrm{mmol}, 202 \mathrm{mg})$ in DMF $(6.1 \mathrm{~mL})$ was prepared under $\mathrm{N}_{2}$. The solid was cooled at room temperature and mixed with the HMDS solution. The final composition of the mixture was $\mathrm{SiO}_{2}: 0.15 \mathrm{HMDS}$ : $10 \mathrm{DMF}$. The suspension was stirred for $2 \mathrm{~h}$ at $120^{\circ} \mathrm{C}$. The material was then filtered, washed with $\mathrm{CH}_{2} \mathrm{Cl}_{2}$ and dried overnight at $100{ }^{\circ} \mathrm{C}$.

Synthesis of silyl-derivative 3P: $0.335 \mathrm{~mL}$ of hexamethylphosphorous triamide was weighted in a flask, previously dried in an oven at $100{ }^{\circ} \mathrm{C}$. The flask containing the reactant underwent $\mathrm{N}_{2} /$ vacuum cycles to ensure the removal of all moisture. 3-(azidopropyl)triethoxysilane $(0.455 \mathrm{~g})$ and dry toluene were added into the mixture, under $\mathrm{N}_{2}$. The mixture was stirred at room temperature for 10 minutes. The mixture was then reacted at $115^{\circ} \mathrm{C}$ for $48 \mathrm{~h}$. Solvent was removed by distillation under reduced pressure.

Grafting of 3P onto MCM-41: $2 \mathrm{~g}$ of MCM-41 were placed in a Schlenck and outgassed at $150{ }^{\circ} \mathrm{C}$ for $2 \mathrm{~h}$. A solution of $3 \mathrm{P}$ in dry toluene $(0.51 \mathrm{~g}$; $0.333 \mathrm{mmol})$ was added and the mixture was stirred at $80{ }^{\circ} \mathrm{C}$ for $1 \mathrm{~h}$. The amount added was $4 \%$ mol of the total amount of silica. Then, triethylamine $(186 \mu \mathrm{L})$ was added and the mixture was stirred at $80{ }^{\circ} \mathrm{C}$ for $24 \mathrm{~h}$. The mixture was filtrated and washed with $\mathrm{CH}_{2} \mathrm{Cl}_{2}$ and acetone and dried overnight. The resulting solid was stirred twice in $0.2 \mathrm{M}$ solution of TMAOH in methanol for $30 \mathrm{~min}$ and dried overnight at $100{ }^{\circ} \mathrm{C}$.

\subsubsection{Characterization}

XRD measurements were carried out using a Philips X'PERT diffractometer equipped with a proportional detector and a secondary graphite monochromator. Data were collected stepwise in the $2^{\circ} \leq 2 \theta \leq 20^{\circ}$ angular region: $0.02^{\circ} 2 \theta$ steps, $20 \mathrm{~s} /$ step accumulation time, beam in produced from $\mathrm{Cu} \mathrm{K}_{\alpha}(\lambda=1.54178 \AA)$. C, N, S, and $\mathrm{H}$ relative amounts were determined using a Carlo Erba 1106 elemental analyzer. Thermogravimetric and differential thermal analyses (TGA-DTA) were conducted in air stream with a Mettler Toledo TGA/SDTA $851 E$ analyzer. $\mathrm{N}_{2}$ adsorption isotherms were measured at $-196{ }^{\circ} \mathrm{C}$ using a Micromeritics ASAP 2010 volumetric adsorption analyzer. Prior to the measurements, samples were outgassed for $12 \mathrm{~h}$ at $100{ }^{\circ} \mathrm{C}$. The BET specific surface area was calculated from the nitrogen physisorption data in the relative pressure range from 0.04 to 0.2 . The total pore volume was obtained from the amount of $\mathrm{N}_{2}$ adsorbed at $\mathrm{P} / \mathrm{P}_{0}$ of about 0.99 . The pore diameter and the pore size distribution were calculated using the Barret-Joyner-Halenda (BJH) method on the adsorption branch of the $\mathrm{N}_{2}$ isotherms. Solid state MAS NMR spectra were recorded at room temperature in a Bruker AV-400 spectrometer. The single pulse ${ }^{29} \mathrm{Si}$ spectra were recorded at $79.5 \mathrm{MHz}$ with a $7 \mathrm{~mm}$ Bruker $B L-7$ probe, using pulses of $3.5 \mu$ s corresponding to a flip angle of $3 / 4 \pi$ radians and a recycle delay of $240 \mathrm{~s} .{ }^{1} \mathrm{H}$ to ${ }^{13} \mathrm{C}$ crosspolarization $(\mathrm{CP})$ spectra were acquired by using a $90^{\circ}$ pulse for ${ }^{1} \mathrm{H}$ of $5 \mu \mathrm{s}$, a contact time of $5 \mathrm{~ms}$, and a recycle of $3 \mathrm{~ms} .{ }^{13} \mathrm{C}$ spectra 
were recorded with a $7 \mathrm{~mm}$ Bruker $B L-7$ probe and at a sample spinning rate of $5 \mathrm{kHz}$. ${ }^{29} \mathrm{Si}$ and ${ }^{13} \mathrm{C}$ were referred to tetramethylsilane and adamantine, respectively. Liquid state ${ }^{13} \mathrm{C}$ NMR spectra were recorded at $75 \mathrm{MHz}$ using a Bruker AMX300 instrument. Chemical shifts are reported in ppm and are referenced to the appropriate residual solvent peak.

\subsubsection{Catalytic tests}

Knoevenagel condensation: The reaction was carried out in high pressure pyrex tubes equipped with magnetic stir bar. $1.0 \mathrm{mmol}$ of benzaldehyde $(106 \mathrm{mg})$ and $1.0 \mathrm{mmol}$ of ethyl cyanoacetate $(113 \mathrm{mg})$ or ethyl acetoacetate $(130 \mathrm{mg})$ or diethyl malonate (160 $\mathrm{mg}$ ), were dissolved in $4 \mathrm{~mL}$ of acetonitrile together with $1 \mathrm{mmol}$ of anisole (internal standard). $10 \mathrm{mmol} \%$ or $2 \mathrm{mmol} \%$ of silica-supported base site were added to the mixture, depending on the experiment. The mixture was refluxed at $80{ }^{\circ} \mathrm{C}$ for $24 \mathrm{~h}$. The reaction was monitored by NMR analysis using $\mathrm{CDCl}_{3}$ as deuterated solvent.

Michael addition: In a $2 \mathrm{~mL}$ glass microreactor equipped with a magnetic stir bar, 1 mmol of ethyl 2-oxocyclopentane 2-carboxylate $(156 \mathrm{mg}), 1 \mathrm{mmol}$ methyl vinyl ketone $(70 \mathrm{mg}), 1 \mathrm{mmol}$ of 4-chlorotoluene (internal standard, $126 \mathrm{mg}$ ) and base catalyst (5 mol $\%$ active site), were mixed and stirred at room temperature in $0.5 \mathrm{ml}$ of $\mathrm{CH}_{3} \mathrm{CN}$ for $3 \mathrm{~h}$. The reaction was monitored by NMR analysis using $\mathrm{d}_{3}-\mathrm{CD}_{3} \mathrm{CN}$ as deuterated solvent.

Aldol condensation furfural-MIBK: Furfural was distilled before each catalytic test. In a $2 \mathrm{~mL}$ glass microreactor equipped with a magnetic stir bar, $1 \mathrm{mmol}$ of furfural $(96 \mathrm{mg})$ $4 \mathrm{mmol}$ methyl isobutyl ketone (400 mg), $1 \mathrm{mmol}$ of 4-chlorotoluene (internal standard, $126 \mathrm{mg}$ ) and base catalyst ( $5 \mathrm{~mol} \%$ active site), were mixed and stirred at $80{ }^{\circ} \mathrm{C}$, in absence of solvent for $24 \mathrm{~h}$. The reaction was monitored by NMR analysis using $\mathrm{d}_{3-}$ $\mathrm{CD}_{3} \mathrm{CN}$ as deuterated solvent. 
A

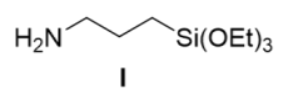
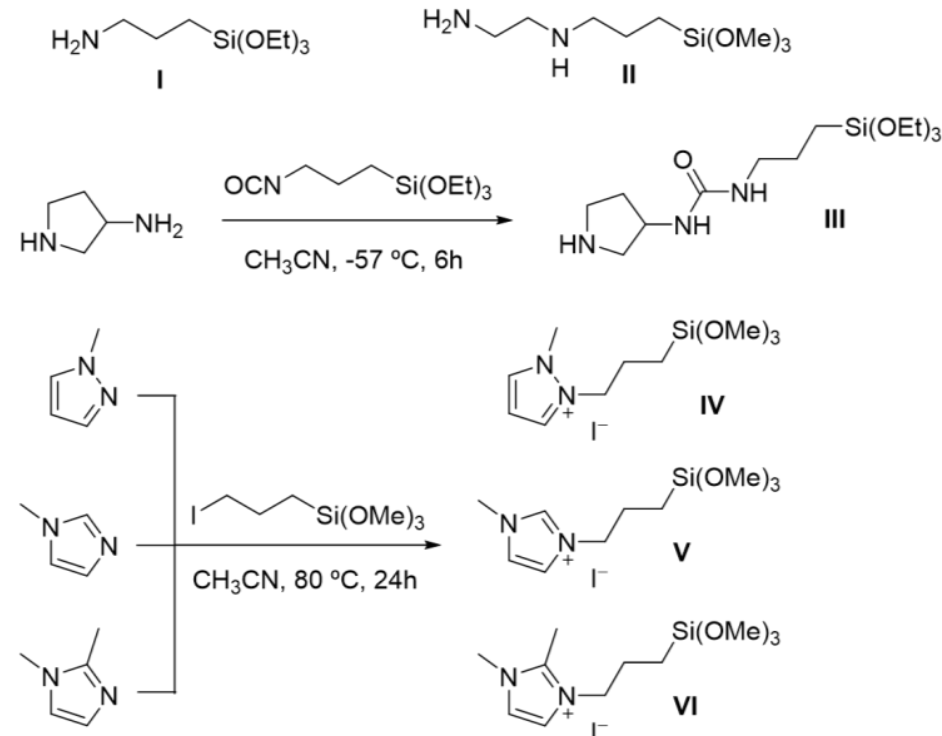

B

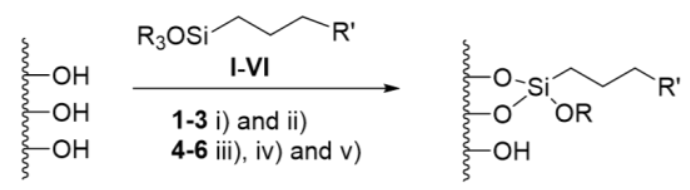

$\mathrm{MCM}-41$

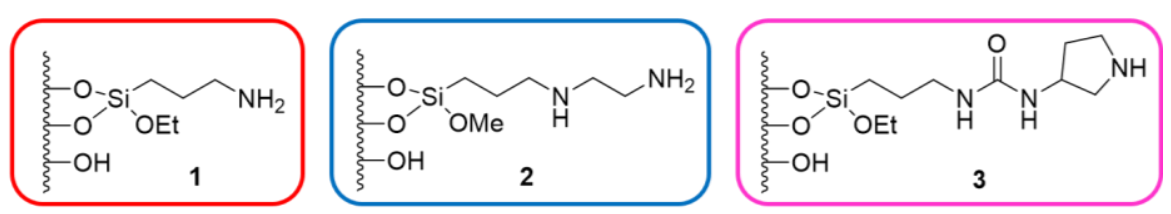

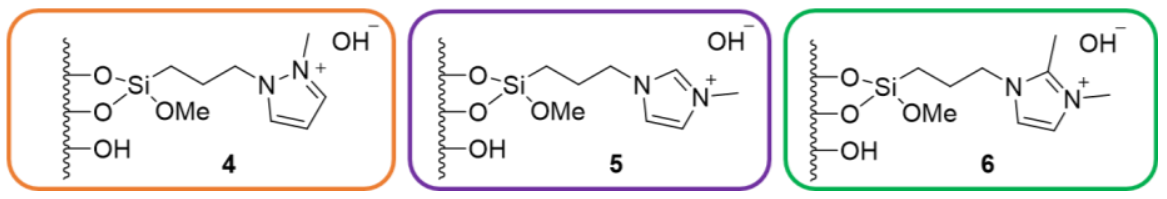

Figure 4.6 A: Scheme of 3-triethoxysilylpropylamine (APTES, I) and N-(2-aminoethyl)-3-(trimethoxysilyl)propylamine (II); synthesis of silyl-derivatives III, IV, V and VI. B: Synthetic steps implemented in the synthesis of silica-supported base cataysts 1-6. i): MCM-41, dry Toluene, 1 h, rT; ii): Compound I, II or III, 24 h, reflux; iii) MCM-41, silylated base precursor IV, V or VI, dry $\mathrm{CH}_{3} \mathrm{CN}, 1 \mathrm{~h}$, rT, iv) TEA, reflux, 24 h; v) TMAOH, $\mathrm{CH}_{3} \mathrm{OH}, 30 \mathrm{~min}$. 


\subsection{Results and discussion}

\subsubsection{Synthesis and characterization of the hybrid base catalysts}

Each hybrid base catalyst was prepared through grafting procedure of silyl-derivatives onto pure silica MCM-41 support. Of the six siloxanes that have been grafted, two of them were purchased (aminopropyl triethox ysilane and N-(2-aminoethyl)-3-(trimethoxysilyl)propylamine, labelled as I and II; see Fig. $4.6 \mathrm{~A}$ ) and four of them were synthesized in the lab (III-VI). In the case of III, the condensation between 2-amino-pyrrolidine and 3-isocyanatopropyltriethoxysilane was performed at $-57{ }^{\circ} \mathrm{C}$. Such low temperature allows the primary amine to react with the isocyanate while inhibiting the reaction of the latter with the secondary amine in the N-heterocycle. IV, V and IV were obtained by thermal induced condensation between 3-iodopropyltrimethoxysilane and 1-methylpyrazole, 1-methylimidazole and 1,2-dimethylimidazole, respectively. The syntheses of silyl-derivatives were followed by liquid $\left({ }^{1} \mathrm{H},{ }^{13} \mathrm{C},{ }^{29} \mathrm{Si}\right) \mathrm{NMR}$. ${ }^{1} \mathrm{H}$ spectra of the custom made silyl-derivatives are reported in Fig. 4.7. In the cases of silyl-derivatives IV, $\mathrm{V}$ and $\mathrm{VI}$, the presence of a positive charge in the azolium ring induced an un-shielding effect on $\mathrm{CH}$ groups. Consequentially, the signals corresponding to such protons were observed at higher chemical shifts, compared to the spectra of corresponding azole rings.

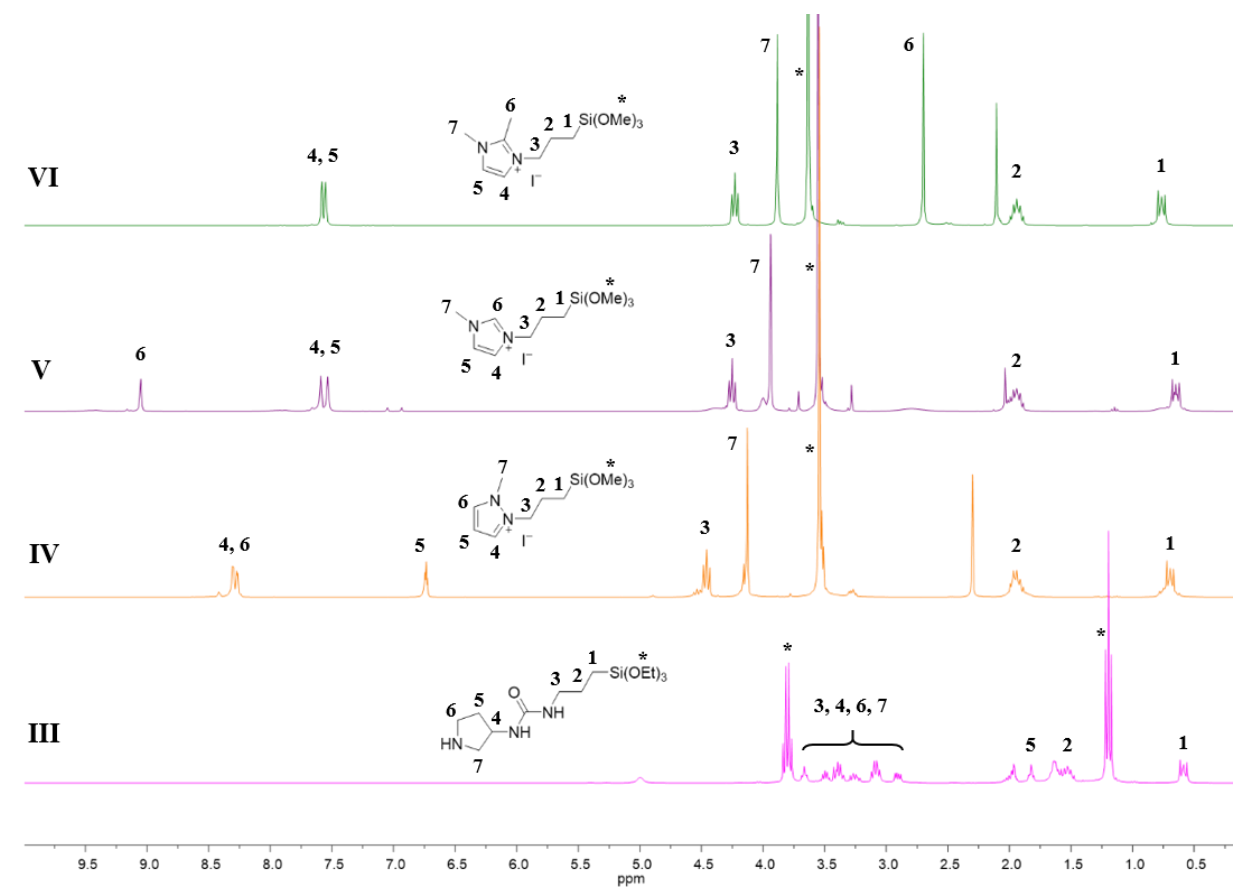

Figure 4.7: ${ }^{1} \mathrm{H}$ NMR spectra of silyl derivatives III, IV, V, and VI. 
${ }^{13} \mathrm{C}$ and ${ }^{29} \mathrm{Si}$ liquid NMR spectra will be discussed further in this Chapter, together with solid state CP/MAS NMR spectra recorded on the hybrids.

Silyl-derivatives I-VI were grafted onto MCM-41 support, following the procedures reported in Section 4.3. Firstly, the hybrids were characterized by X-ray diffraction (Fig. 4.8). The diffractograms of all samples exhibit the (100) reflection peak at $2 \theta$ between 2.3 and $2.6^{\circ}$ and the (110) and (200) reflections in the $2 \theta$ range between 3.8 and $4.8^{\circ}$, confirming the preservation of the 2D hexagonal structure, typical of the M41S materials, throughout the grafting procedure and the exchange at alkaline $\mathrm{pH} .{ }^{21}$ It can be noticed the position of the (100) peak slightly differs between the samples.

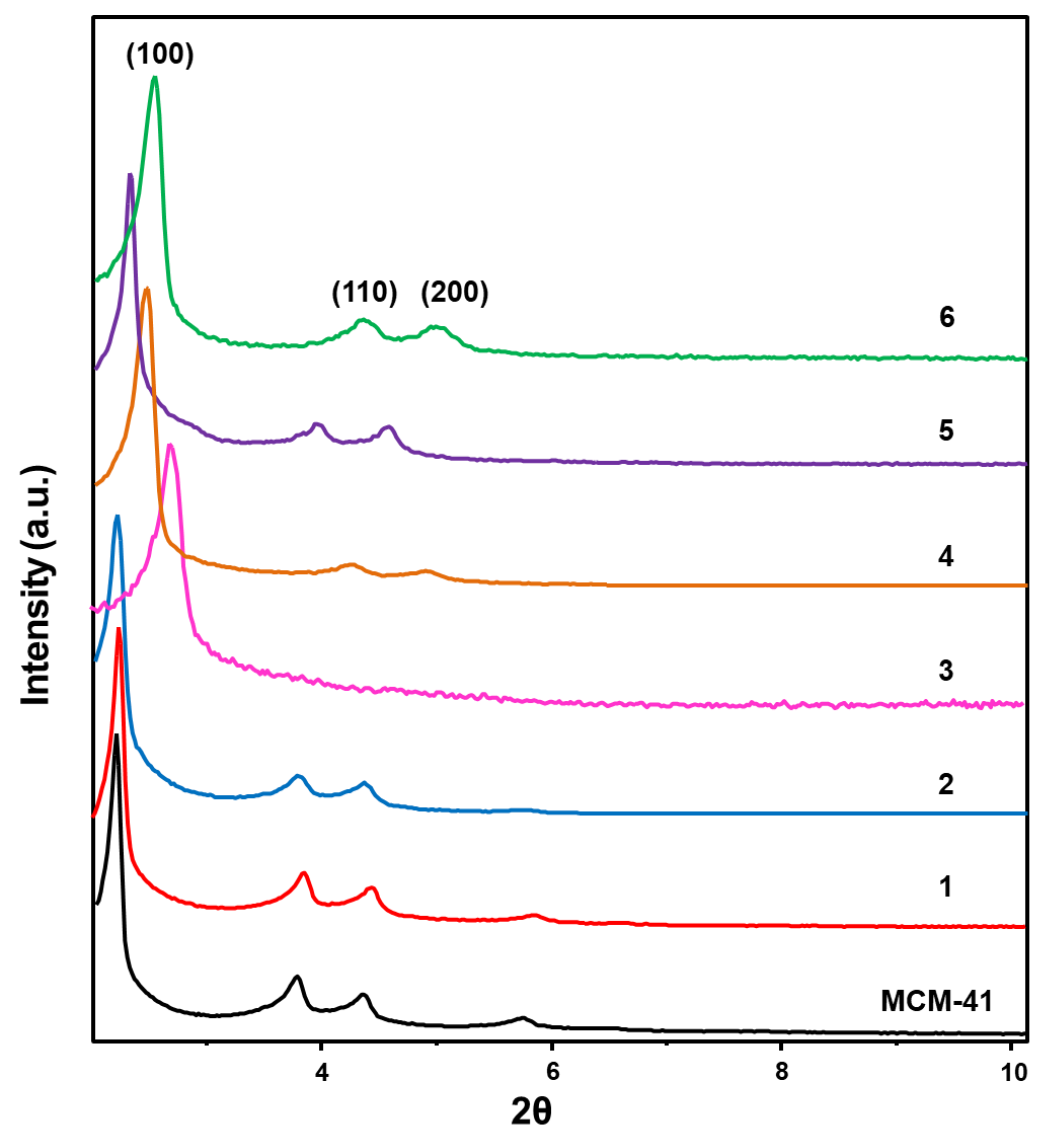

Figure 4.8: Powder XRD patterns of the MCM-41 support and the silica-supported base catalysts 1-6. 
This is partly due to differences is the size of the pending moieties, which affect the mean pore diameter of the hybrids to a different extent and therefore cause a displacement of the peak that is proportional to the size of the pending moieties. However, a full understanding of these features should keep into account the bending of the organic moieties and their interaction with the surface of the support.

The presence of the silylated base precursors in the composition of hybrid materials was firstly accessed by means of elemental analysis. The $\mathrm{C}, \mathrm{H}, \mathrm{N}$ contents and the relative weight percentage of the organic moieties present in the silica-supported base materials are reported in Table 4.1. The organic content of the organosiliceous materials appears to be proportional to the molecular weight of the silylated base precursor. Accordingly, the highest organic content corresponds to catalyst $3(10.4 \%)$, the molecular weight of the organic moiety III being $333.5 \mathrm{~g} / \mathrm{mol}$, while the smallest organic content corresponds to catalyst $1(4.4 \%)$, where the molecular weight of the organic moiety I is $221.37 \mathrm{~g} / \mathrm{mol}$. Coherently, the relative organic content for catalyst 4 and 5 is practically the same ( 8.3 and $8.6 \%$, respectively), being the molecular weight of both organic moieties IV and V $362.38 \mathrm{~g} / \mathrm{mol}$. Quantifying the amount of $\mathrm{N}$ allowed for the determination of the amount of active sites per gram of solid. Minor differences in the active base sites loading were observed between the six catalysts.

Thermogravimetric analysis was also performed to gain insights about the thermal stability of organic species present in the hybrid materials. Weight losses (TGA) and derivative curves (DTA) for the organosilylated base catalysts 1-6 are shown in Fig. 4.9. For all curves, a first weight loss due to the removal of physisorbed water is observed at around $90-150{ }^{\circ} \mathrm{C}$. In the range between $200-400{ }^{\circ} \mathrm{C}$, the decomposition of the organic moieties takes place for all samples. A progressive weight loss at temperatures above $500{ }^{\circ} \mathrm{C}$ is detected due to dehydroxylation phenomena, associated with the condensation of external silanol groups. Consequentially, release of water molecules occurs and strained siloxane bridges form.

Table 4.1: Organic content in the mesoporous hybrid materials.

\begin{tabular}{ccccc}
\hline Catalysts & N\% & C\% & Organic content $(\mathrm{EA}) \%$ & $*$ Active sites $(\mathrm{mmol} / \mathrm{g})$ \\
\hline $\mathbf{1}$ & 1.1 & 3.3 & 4.4 & 0.79 \\
$\mathbf{2}$ & 2.4 & 5.8 & 8.2 & 0.86 \\
$\mathbf{3}$ & 2.5 & 7.9 & 10.4 & 0.60 \\
$\mathbf{4}$ & 1.9 & 6.3 & 8.3 & 0.68 \\
$\mathbf{5}$ & 2.0 & 6.6 & 8.6 & 0.71 \\
$\mathbf{6}$ & 2.0 & 7.3 & 9.3 & 0.71 \\
\hline
\end{tabular}

$*$ active sites $=\mathrm{N} \% / \mathrm{n}_{\mathrm{N}} / 14 / 100 . \mathrm{n}_{\mathrm{N}}=$ number of $\mathrm{N}$ atoms present in the hybrid materials. 


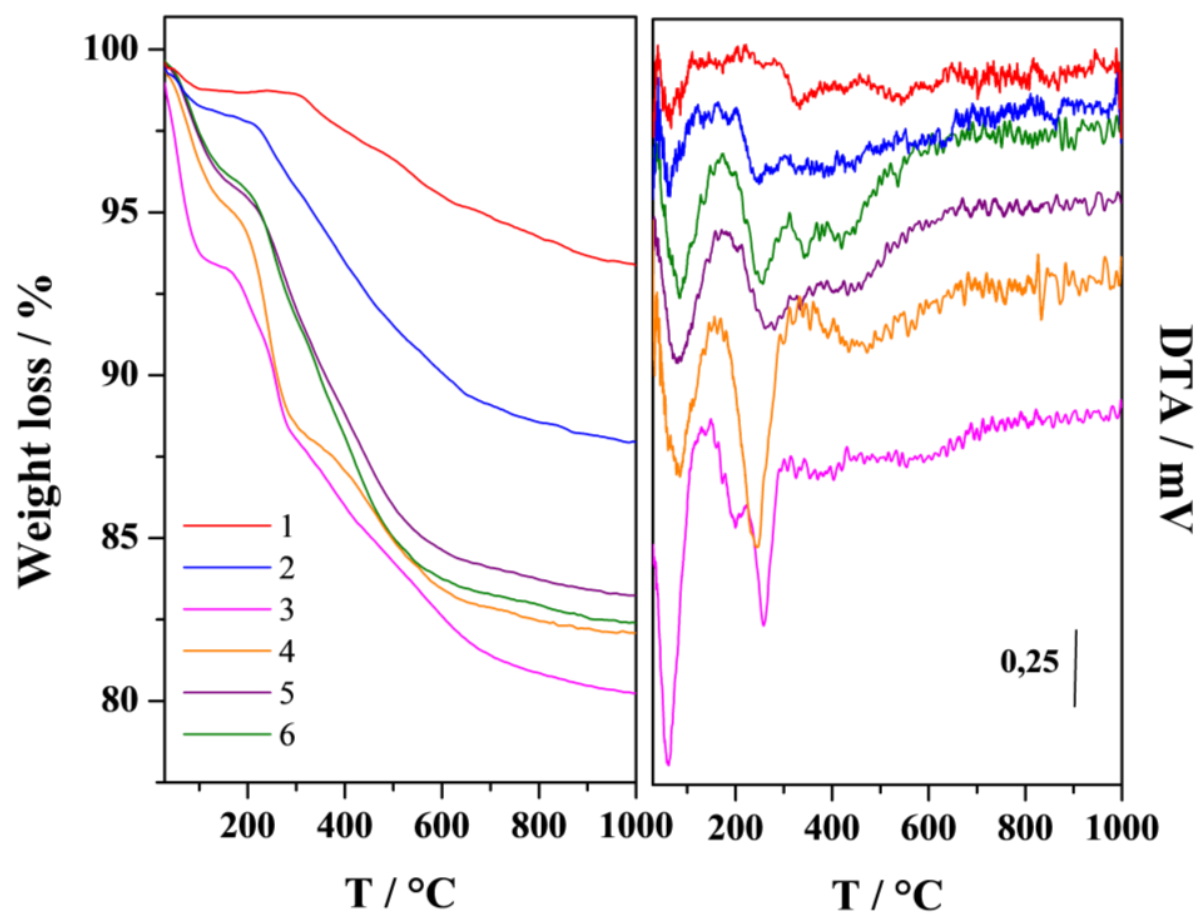

Figure 4.9: TGA and DTA curves of the hybrid base catalysts 1-6.

The integrity of the organic species in the final hybrid catalysts and the covalent anchoring of the silylated base precursors onto the MCM-41 support was studied by means of solid state MAS NMR spectroscopy. ${ }^{13} \mathrm{C}$ CP/MAS NMR spectra were recorded on all hybrid base materials and compared to the liquid NMR spectra recorded on the silylderivatives before the grafting (Fig. 4.10). First, ${ }^{13} \mathrm{C}$ signals present in the liquid NMR spectra confirm the structure of the silyl-derivatives prepared (compounds III-VI). Secondly, solid state spectra of the hybrids show signals coherent with the target silyl-derivatives, confirming the integrity of the organic moieties after grafting and exchange processes. Specifically, ${ }^{13} \mathrm{C}$ signals due to the propyl chains are observed in the range 0 - $70 \mathrm{ppm}$ for 1-6. Signals related to other aliphatic carbon atoms of catalyst 2 and 3 appear in the same range, with the exception of a signal present in the spectrum of catalyst 3, at $160 \mathrm{ppm}$, which is the characteristic signal of the carbonyl carbon, present in the urea group. Spectra of catalysts 4, 5 and 6 show a signal due to the methyl group N$\mathrm{CH}_{3}$ in the range between 33 and $36 \mathrm{ppm}$. 


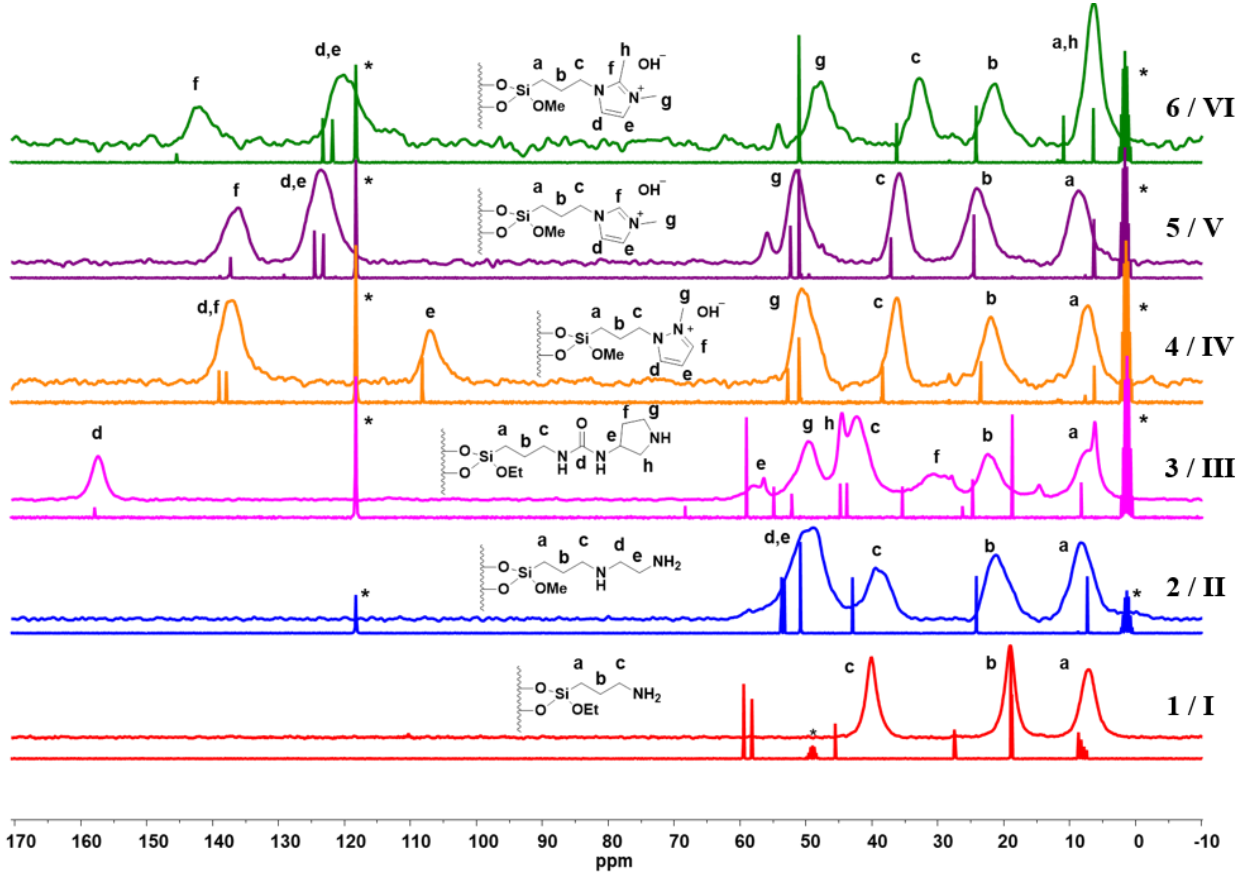

Figure 4.10: ${ }^{13} \mathrm{C}$ CP/MAS NMR spectra of the hybrid base catalysts 1-6 and ${ }^{13} \mathrm{C}$ liquid NMR spectra of silyl-derivatives I-VI. *Solvent residual peak.

Other aromatic signals related to the azol rings are detected within the expected range of 105-145 ppm. Specific assignation of each signal are reported in Fig. 4.10, for each spectrum. ${ }^{29}$ Si MAS NMR spectra of hybrids 1-6 (Fig. 4.11) exhibit signals in the range between -50 to $-80 \mathrm{ppm}$ ascribed to T-type silicon species, i.e. silicon atoms directly bounded to carbon atoms (Schematic representation reported in Chapter 3, Fig. 3.14 A), confirming the successful incorporation of the silyl-derivatives through reaction of the alkoxy-silyl groups of the organic precursors and the silanol groups present onto the surface of the inorganic support. Comparison between ${ }^{29} \mathrm{Si}$ solid state NMR spectra of the hybrids and liquid NMR spectra of the pure silyl-derivatives used as precursors (Fig. 4.11, insets) further confirms the anchoring of the organic molecules onto the surface of mesoporous silica: Silyl-derivatives I, II, V and VI exhibit a signal at around $-43 \mathrm{ppm}$ which, after the grafting, is shifted in the range between -50 and $-80 \mathrm{ppm}$, as hydrolysis/condensation of the alkoxy-silyl groups cause a change in the chemical make-up of the silicon atoms of the precursors, with consequent change in their chemical shift. The presence of the support is seen from the Q-type signals related to bulk and surface silicon atoms. Specifically, three peaks at $-92,-100$ and $-110 \mathrm{ppm}$ are observed, due to $\mathrm{Q}^{2}\left(\mathrm{Si}(\mathrm{OH})_{2}(\mathrm{OSi})_{2}\right), \mathrm{Q}^{3}\left(\mathrm{Si}(\mathrm{OH})(\mathrm{OSi})_{3}\right)$ and $\mathrm{Q}^{4}\left(\mathrm{Si}(\mathrm{OSi})_{4}\right)$ silicon species, respectively. ${ }^{22}$ 


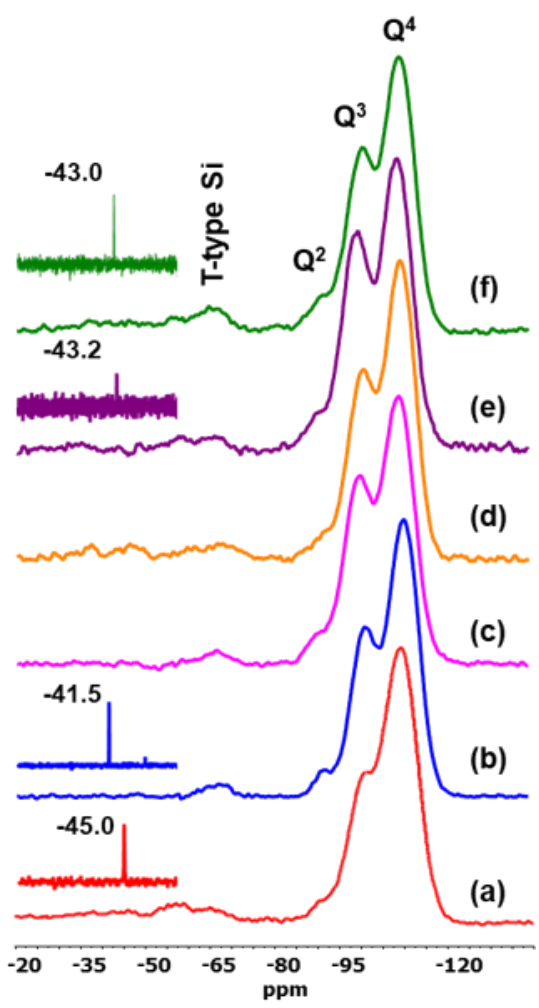

Figure 4.11: ${ }^{29} \mathrm{Si}$ BD/MAS NMR spectra of hybrid base catalysts 1-6 and ${ }^{29} \mathrm{Si}$ liquid NMR spectra of silyl-derivatives I, II, V and VI.

Integration of $\mathrm{T}$ and $\mathrm{Q}$ peaks in the ${ }^{29} \mathrm{Si} \mathrm{BD} / \mathrm{MAS} \mathrm{NMR}$ spectra was performed to calculate the $\mathrm{T} /(\mathrm{Q}+\mathrm{T})$ ratio, which allowed to estimate the number of functionalized silicon atoms in the hybrids (Table 4.2). According to the estimate calculated through the Sifund $/ \mathrm{Si}_{\text {tot }}$ ratio, the percentage of grafting achieved in this catalysts composition was between 1.9 and $4.7 \mathrm{~mol} \%$, respect to total moles of silica. 
Table 4.2: Speciation of silicon atoms in hybrid materials 1-6.

\begin{tabular}{cc}
\hline Catalysts & $\mathrm{Si}_{\text {fund }} / \mathrm{Si}_{\text {tot }}(\mathrm{NMR})$ \\
\hline $\mathbf{1}$ & 0.047 \\
$\mathbf{2}$ & 0.023 \\
$\mathbf{3}$ & 0.019 \\
$\mathbf{4}$ & 0.023 \\
$\mathbf{5}$ & 0.020 \\
$\mathbf{6}$ & 0.042 \\
\hline
\end{tabular}

FTIR spectra were recorded on the hybrid materials, after outgassing for $2 \mathrm{~h}$ at $120{ }^{\circ} \mathrm{C}$ (Fig. 4.12). Focusing first on the high frequency region of the spectra, a signal centered at $3740 \mathrm{~cm}^{-1}$ is present in all six catalysts and is ascribed to the stretching modes of isolated silanols. A broad signal centered in the range between 3700 and $3500 \mathrm{~cm}^{-1}$ is bestowed to the stretching modes of hydroxyl groups in interaction with each other through H-bond. Moreover, in the ionic hybrids (catalysts 4-6), a signal of this multicomponent band stands out at about $3650 \mathrm{~cm}^{-1}$, attributed to the stretching modes of the hydroxyl anions present as counter ion. Spectra of catalysts 1 and 2 show two signals centered at about 3370 and $3310 \mathrm{~cm}^{-1}$ related to the asymmetric and symmetric stretching modes of the $\mathrm{N}-\mathrm{H}$ bond, respectively. In the same region of the spectrum of catalyst 3 , a multi-component signal is observed between 3500 and $3260 \mathrm{~cm}^{-1}$, related to the overlapping of signals arising from the $\mathrm{N}-\mathrm{H}$ stretching modes in the urea group (about $3440 \mathrm{~cm}^{-1}$ ) and in the pyrrolidine ring (asymmetric at $3365 \mathrm{~cm}^{-1}$; symmetric at 3300 $\left.\mathrm{cm}^{-1}\right)$. Spectra of hybrid 4-6 feature a multi-component signal between 3200 and 3000 $\mathrm{cm}^{-1}$, ascribed to the alkene $\mathrm{C}-\mathrm{H}$ stretching modes, in the azo-cycles. In the region comprised between 3000 and $2840 \mathrm{~cm}^{-1}$, peaks related to the aliphatic $\mathrm{C}-\mathrm{H}$ stretching modes are observed in all six spectra. ${ }^{23}$ Due to the similar chemical structure, the low frequency region of the spectra recorded on catalyst 1 and 2 show resembling features: a signal placed at $1590 \mathrm{~cm}^{-1}$ due to the primary amine $\mathrm{N}-\mathrm{H}$ bending modes is present in both spectra; additionally, a weak signal at $1460 \mathrm{~cm}^{-1}$ is detected and ascribed to the scissor vibration modes of the $\mathrm{CH}_{2}$ groups of the propyl linker chain. In the spectrum of catalyst 3 , two intense and partially overlapped signals are visible at 1635 and $1605 \mathrm{~cm}^{-1}$. The first one is due to the stretching modes of the carbonyl $\mathrm{C}=\mathrm{O}$ bonds in the urea group (amide I band), while the second one is related to the amide II band, i.e. a motion combining C-N stretching and N-H bending of the amide group. Furthermore, a band at 1535 $\mathrm{cm}^{-1}$ is observed and tentatively ascribed to the $\mathrm{N}-\mathrm{H}$ bending modes in the pyrrolidine ring. Signals related to the bending modes of $\mathrm{R}-\mathrm{C}-\mathrm{H}$ species (where $\mathrm{R}$ is either a carbon or a nitrogen atom) are observed in the region between 1490 and $1370 \mathrm{~cm}^{-1}$. 


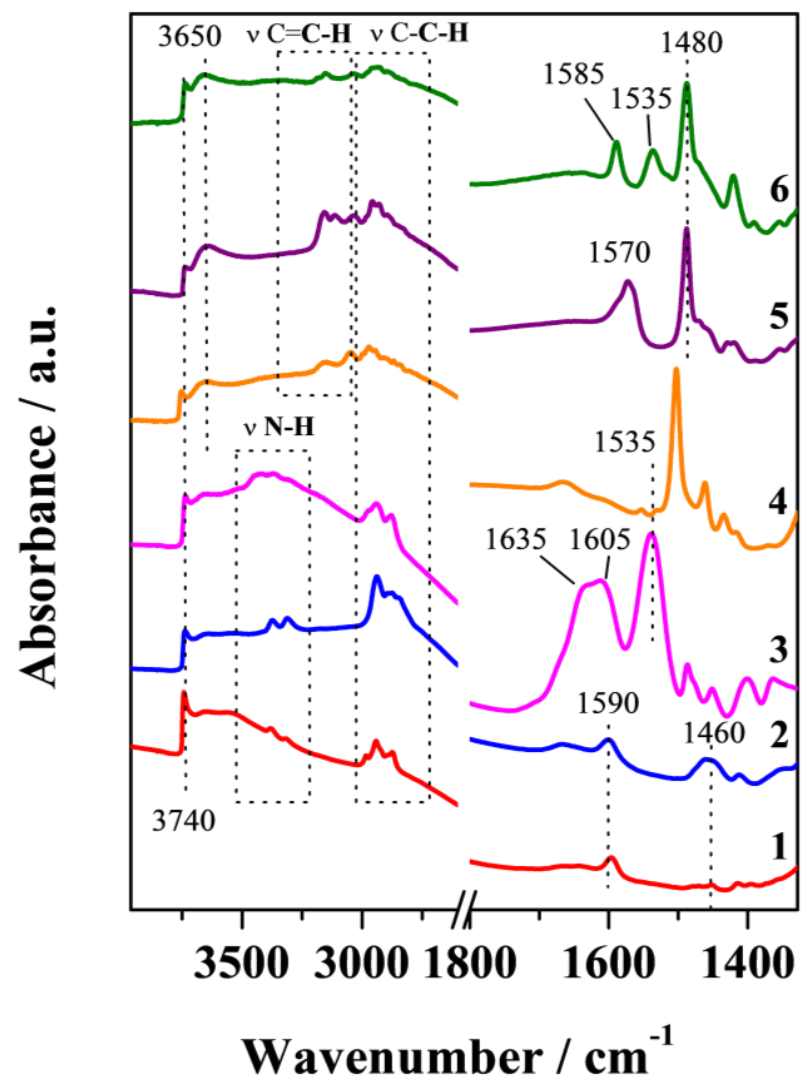

Figure 4.12: FTIR spectra of hybrid materials 1-6 after outgassing at $120{ }^{\circ} \mathrm{C}$.

Due to the complex nature of the pyrazolium ion and to the lack of spectroscopic data reported in the literature, no specific assignation of the signal observed in the low frequency region of spectrum 4 was possible. The low frequency region of the spectra recorded on the imidazolium-derivative catalysts (5 and 6) show similar spectroscopic features. A signal due to a motion combining C4-C5 and C-N stretching is present at 1570 $\mathrm{cm}^{-1}$ in spectrum 5, and $1585 \mathrm{~cm}^{-1}$ in spectrum 6 . Additionally, a signal centered at about $1480 \mathrm{~cm}^{-1}$ is bestowed to the $\mathrm{CH}_{3}(\mathrm{~N} 1)$ scissoring modes. The spectrum of hybrid 6 also show a typical band related to a motion combining N1-C2-N3 stretching and $\mathrm{CH}_{3}(\mathrm{C} 2)$ rocking modes, centered at about $1535 \mathrm{~cm}^{-1} \cdot 24$

$\mathrm{N}_{2}$ physisorption isotherms at $77 \mathrm{~K}$ were recorded on hybrid catalysts 1-6 and on bare MCM-41 support, to study their textural properties (Table. 4.3). 
Table 4.3: Textural properties of the hybrid base catalysts 1-6.

\begin{tabular}{ccc}
\hline Catalysts & SSABET $\left(\mathrm{m}^{2} \mathrm{~g}^{-1}\right)$ & $\begin{array}{c}\text { Pore volume } \\
\left(\mathrm{cm}^{3} \mathrm{~g}^{-1}\right)\end{array}$ \\
\hline $\mathbf{1}$ & 973 & 0.47 \\
$\mathbf{3}$ & 1159 & 0.55 \\
$\mathbf{4}$ & 1005 & 0.43 \\
$\mathbf{5}$ & 998 & 0.45 \\
$\mathbf{6}$ & 1023 & 0.46 \\
$\mathrm{MCM}-41$ & 1038 & 0.44 \\
\end{tabular}

In all cases, the isotherms show a type IV behavior, with a hysteresis loop, typical of mesoporous materials (Fig. 4.13 A). The $\mathrm{N}_{2}$ uptake is consistent with MCM-41-type materials.
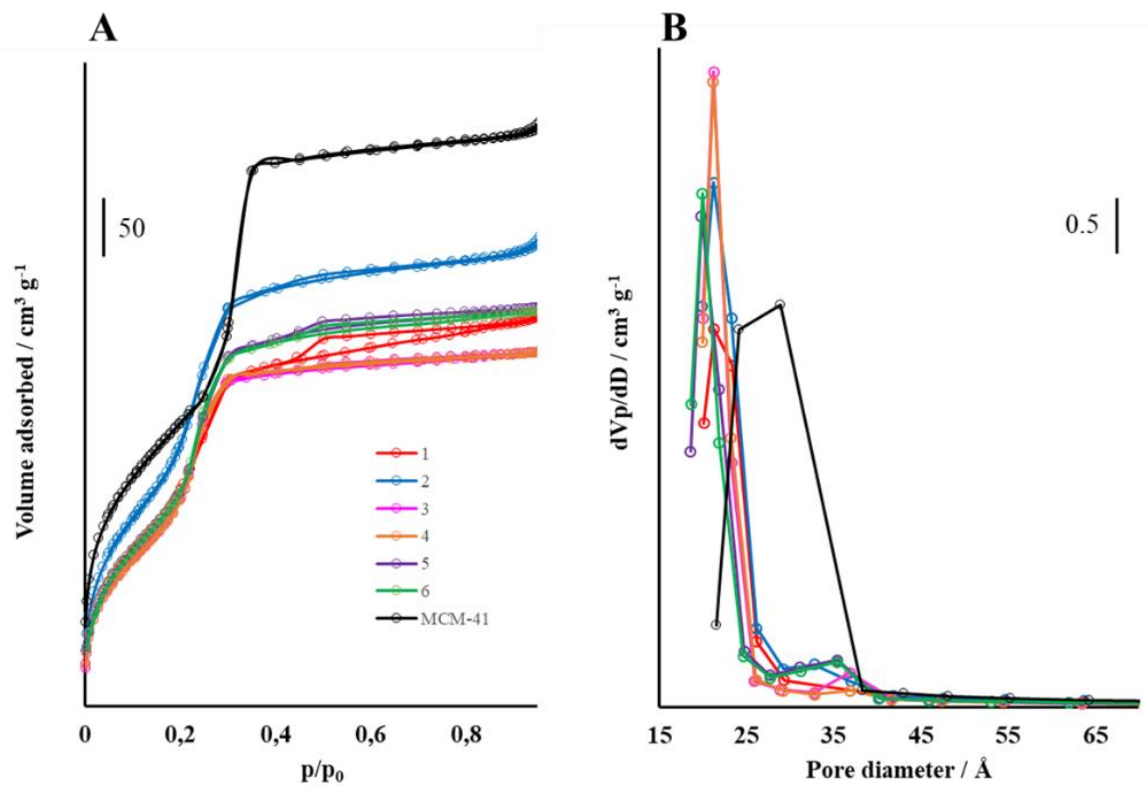

Figure 4.13 A: $\mathrm{N}_{2}$ adsorption isotherms of the hybrid catalysts 1-6. B: BJH pore size distribution of catalysts 1-6. 
Specifically, all hybrid catalysts feature an isotherm with an inflexion point within the range of $\mathrm{p} / \mathrm{p}_{0} 0.2-0.3$ whereas the inflexion point of the bare silica support is observed at higher $\mathrm{p} / \mathrm{p}_{0}$ value. This means that the pore diameter of the support is slightly larger than that of the hybrids materials, as a consequence of a partial reduction of the pore aperture due to the incorporation of the organic moieties within the mesopores. By analyzing the isotherms using the Brunauer-Emmett-Teller (BET) method, specific surface areas (SSA) between 844 and $1159 \mathrm{~m}^{2} / \mathrm{g}$ were observed. The pore volume of the hybrids and of the purely inorganic support were obtained by Barrett-Joyner-Halenda (BJH) analysis of the isotherms (Table 4.3). Upon grafting, the BJH pore volume drops from 0.8 to 0.4$0.5 \mathrm{~cm}^{3} / \mathrm{g}$, due to the presence of the pendant organic moieties, which partially block the pore volume. The BJH pore size distribution (Fig. $4.13 \mathrm{~B}$ ) confirms that the grafting of the organic moieties caused a partial decrease in the mean pore diameter.

\subsubsection{Catalytic activity}

The activity of hybrid base catalysts 1-6 was tested in the condensation of carbonyl compounds with active methylenic substrates. Knoevenagel condensation and Michael addition were used as model reactions. These reactions are a key tool in organic synthesis for $\mathrm{C}-\mathrm{C}$ bond formation and in the synthesis of substituted alkene products, that are of great interest in the production of perfumes, drugs, dyes and polymers. ${ }^{25-28}$

\subsubsection{Knoevenagel condensation}

This reaction can be catalyzed by weak and strong bases, depending on the reactivity of the methylenic-activated substrate, which makes it an appropriate model reaction for comparing catalysts with different basicity. The kinetics of the Knoevenagel condensation is generally considered to be a first order reaction with respect to each reactant and the catalyst. ${ }^{29,30}$

The catalytic performances of hybrid base catalysts 1-6 were evaluated in the Knoevenagel condensation between benzaldehyde and substrates featuring different $\mathrm{pKa}$ values (due to the different activated methylenic groups), such as ethyl cyanoacetate $(\mathrm{pKa} \approx 9)$, ethyl acetoacetate $(\mathrm{pKa} \approx 11)$ and diethyl malonate $(\mathrm{pKa} \approx 13)$ (Fig. 4.14, Table 4.4). Moreover, one of the ionic catalysts was tested before exchanging of $\mathrm{I}^{-}$counterions for $\mathrm{OH}^{-}$(catalyst 6, Table 4.4, entry 6-I). Finally, the effect of the surface silanols on the activity of the catalysts was explored by passivating the surface of 1 with hexamethyldisilazane (HMDS) and testing the resulting material as base catalyst (1-HMDS).

Neutral base catalysts 1-3 and ionic base catalysts 4-6 showed high catalytic activity in the Knoevenagel condensation between benzaldehyde and the least demanding substrate, ethyl cyanoacetate. The reactions were performed with an active site loading of $10 \mathrm{~mol} \%$. 


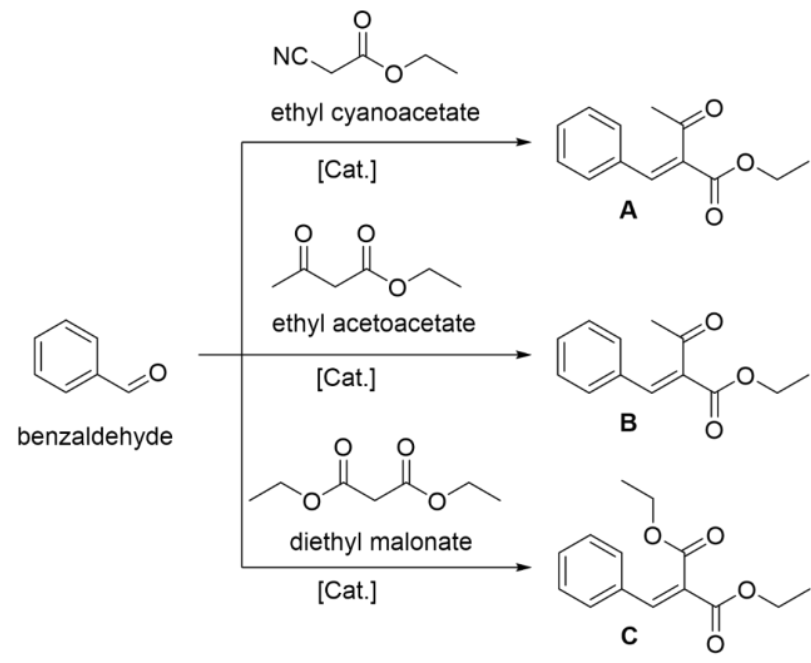

Figure 4.14: Schematic representation of the Knoevenagel reaction of benzaldehyde and ethyl cyanoacetate, ethyl acetoacetate and diethyl malonate.

Table 4.4: Knoevenagel condensation between benzaldehyde and ethyl cyanoacetate (A), ethyl acetoacetate (B) and diethyl malonate $(C)$.

\begin{tabular}{cccc}
\hline Catalyst & $\begin{array}{c}\text { Yield (\%) } \\
\mathbf{A}^{\mathrm{a}}\end{array}$ & $\begin{array}{c}\text { Yield }(\%) \\
\mathbf{B}^{\mathrm{d}}\end{array}$ & $\begin{array}{c}\text { Yield }(\%) \\
\mathbf{C}^{\mathrm{d}}\end{array}$ \\
\hline $\mathbf{1}$ & 93 & 65 & 45 \\
$\mathbf{3}$ & 90 & 63 & 22 \\
$\mathbf{4}$ & 95 & 46 & 18 \\
$\mathbf{5}$ & 91 & 0 & 0 \\
$\mathbf{6}$ & 89 & 0 & 0 \\
$\mathbf{1 - H M D S}$ & 91 & 0 & - \\
MCM-41 $^{\mathrm{b}}$ & 21 & - & - \\
$\mathbf{6 - I}^{\mathrm{c}}$ & 20 & - & - \\
None & 19 & - & - \\
\hline
\end{tabular}

${ }^{\text {a }}$ Reaction conditions: benzaldehyde $(1 \mathrm{mmol})$, ethyl cyanoacetate $(1 \mathrm{mmol})$, ethyl acetoacetate $(1$ mmol), diethyl malonate $(1 \mathrm{mmol}) . \mathrm{CH}_{3} \mathrm{CN}(4 \mathrm{ml}), 80^{\circ} \mathrm{C}, 0.5 \mathrm{~h}, 10 \mathrm{~mol} \%$ of base sites. ${ }^{\mathrm{b}} 200 \mathrm{mg}$ of MCM-41, 24 h. $^{\mathrm{c}} 2 \mathrm{~h},{ }^{\mathrm{d}} 24 \mathrm{~h}$. 
Quantitative yields of the final product A were reached after 30 min, with $100 \%$ selectivity (Fig. 4.15). A catalytic test using hydrophobic catalyst 1-HMDS highlights the importance of the weakly acidic surface silanol groups on the activity of the hybrid. A pronounced decrease in the catalytic activity, down to $21 \%$ yield, is observed after 30 minutes, in line with the premises described in the introduction of this Chapter. It was detected that $24 \mathrm{~h}$ reaction time is needed to obtain quantitative yields. Additionally, when the reaction was performed using bare MCM-41 as catalyst, only a $20 \%$ yield was observed after $24 \mathrm{~h}$, which reveals that weakly acidic silanols are mildly active in catalyzing this particular Knoevenagel condensation, even without the cooperation with the base active site.

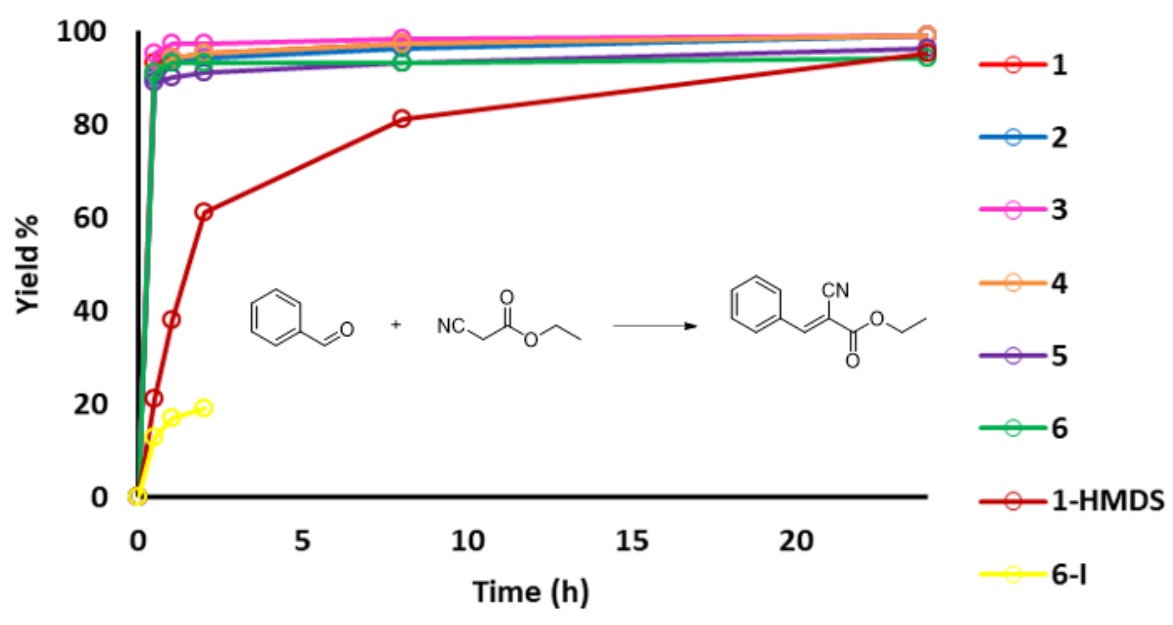

Figure 4.15: Catalytic activity of the hybrid base catalysts $(10 \% \mathrm{~mol})$ in the Knoevenagel condensation between benzaldehyde and ethyl cyanoacetate.

Catalyst 6-I was tested in the Knoevenagel condensation of ethylcyanoacetate with benzaldehyde. The pendant organic species seem to be ineffective in promoting the reaction, with only $19 \%$ yield observed after $2 \mathrm{~h}$, the same was detected when using pure silica MCM-41. This result highlights the importance of having the hydroxide group as counter-ion in ionic base catalysts. A blank experiment was carried out in absence of any catalyst, with no conversion observed.

Knoevenagel condensation reactions between benzaldehyde and two substrates featuring higher $\mathrm{pKa}$, i.e. ethyl acetoacetate and diethyl malonate, were performed in presence of the hybrid base catalysts with a loading of $10 \mathrm{~mol} \%$. The catalytic tests showed moderate activity of the neutral base catalysts $1-3$. (Table 4.4; Fig. 4.16). The results indicate that 
the most performing catalyst was 1 , featuring propyl amine residues, and that ethyl acetoacetate $(\mathrm{pKa} \approx 11)$ was more easily activated than diethyl malonate $(\mathrm{pKa} \approx 13)$ with a $65 \%$ yield of product $\mathrm{B}$ and a $45 \%$ yield of product $\mathrm{C}$ after $24 \mathrm{~h}$. On the other hand, ionic base catalysts 4-6 did not show any activity in these more demanding Knoevenagel condensation reactions. This finding is consistent with an imine/enamine (or iminium) mechanism through which the reaction proceeds when catalyzed by primary (or secondary) amines. ${ }^{14}$

A

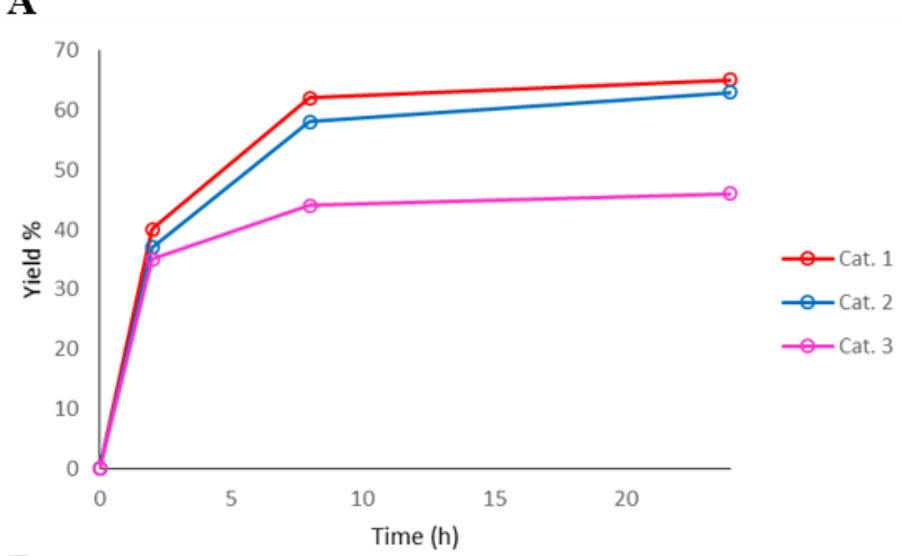

B

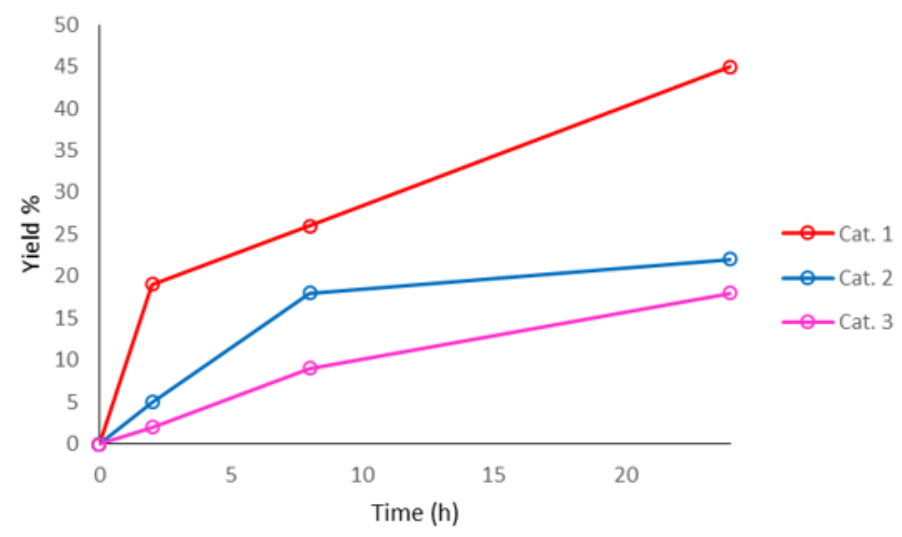

Figure 4.16: Catalytic activity of the hybrid base catalysts 1-3 (10 mol \% catalyst loading) in the Knoevenagel condensation of benzaldehyde with ethyl acetoacetate (A) and diethyl malonate (B). 
A proposed imine mechanism promoted by surface silanol groups is reported in Fig. 4.17 , taking catalyst 1 and ethyl cyanoacetate as examples. The catalytic cycle begins with a molecule of benzaldehyde interacting and forming a $\mathrm{H}$-bond with a free silanol group through its carbonyl oxygen. Then, the free electron pair of the primary amine attacks the activated carbonyl group of the benzaldehyde molecule, forming carbinolamine through a proton-transfer. Next, the carbinolamine loses a $\mathrm{H}_{2} \mathrm{O}$ molecule, yielding an imine intermediate. The methylene carbon of an ethyl cyanoacetate molecule is deprotonated by a second base site yielding a stabilized enolate. The enolate then attacks the imine group forming a new carbon-carbon bond. Finally, a rearrangement regenerates the catalyst, yielding the final olefin product.

A few additional considerations need to be made, considering other reactions that can occur, besides those depicted in the main catalytic cycle, reported in Fig. 4.17. Firstly, the activation of benzaldehyde can occur through the formation of iminium ion when the catalyst features a secondary amine (Cat. 2 and 3). Secondly, ethyl acetoacetate can be activated through the formation of imine and iminium ions, when reacting with primary and secondary amines, respectively (Cat. 1, 2 and 3). This might explain why Cat. 2 showed similar activity to that of Cat. 1 in the reaction between benzaldehyde and ethyl acetoacetate, while showing much lower activity than the primary amine in the reaction between benzaldehyde and diethyl malonate, in which this mechanism was not possible.

In light of the reaction mechanism reported and considering the results of the catalytic tests, the reaction between benzaldehyde and diethyl malonate can provide some key insights for contextualizing the differences in activity among the catalysts. The only possible mechanism for the activation of diethyl malonate is the acid-base interaction (deprotonation). Ionic catalysts are more basic than non-ionic ones and are then expected to be more efficient in this process. However, the reaction of benzaldehyde with supported amines and consequent formation of imine (or iminium) creates a much more electrophilic species than can react with the enolate much faster than when only benzaldehyde is present in the reaction mixture, pushing the equilibrium of the acid-base interaction toward the formation of the enolate.

Besides increasing the reactivity of carbonyl groups through $\mathrm{H}$-bond interactions, silanol groups play a key role also in the adsorption of the substrate on the surface, which increases the local concentration and, as a consequence, the reaction rate. ${ }^{26,31,32}$ 


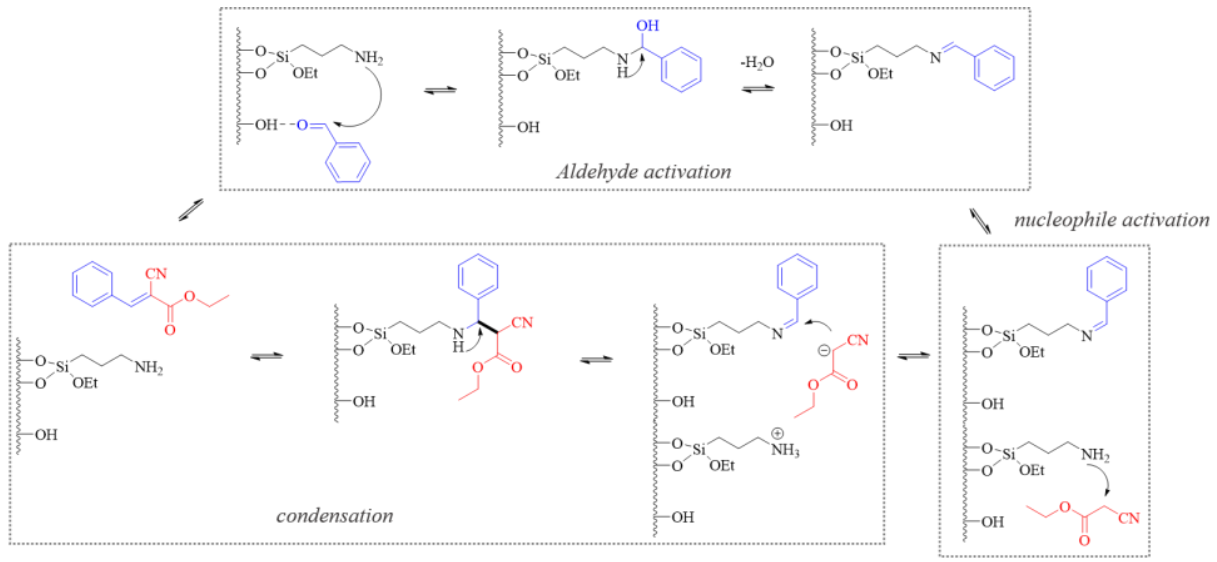

Other reactions

Aldehyde activation

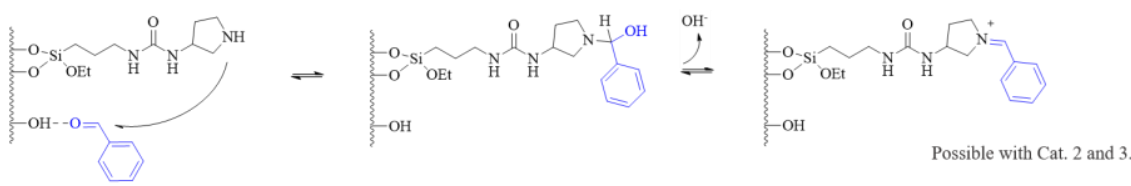

Nucleophile activation

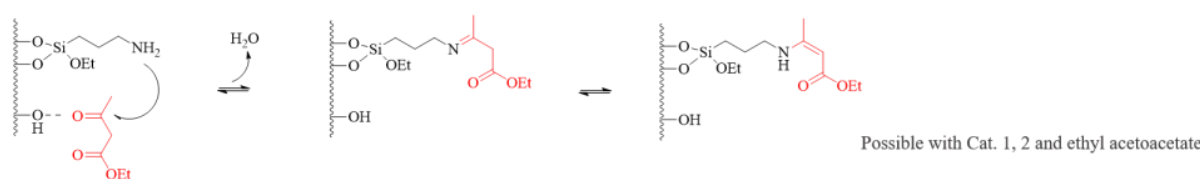

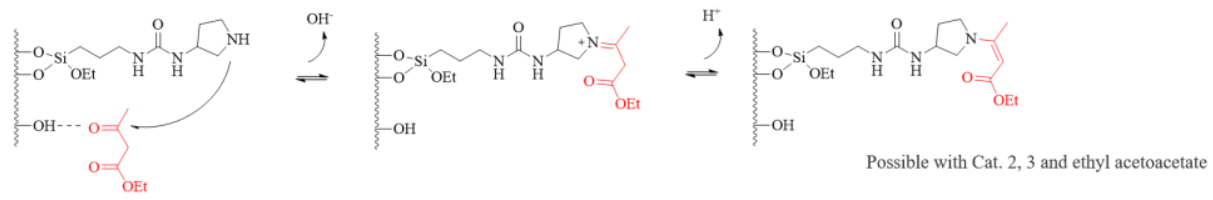

Condensation

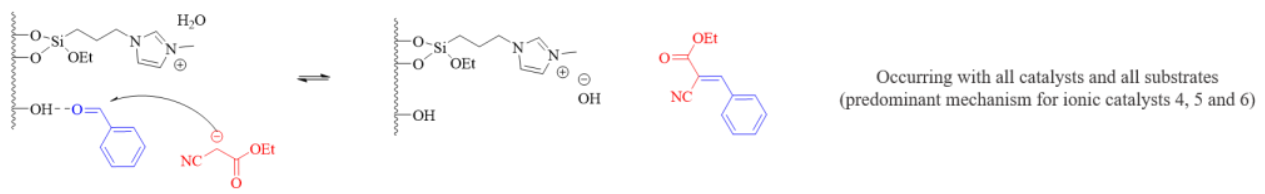

Figure 4.17: Proposed catalytic cycle of the Knoevenagel reaction of benzaldehyde and ethyl cyanoacetate and additional reactions that can occur. 
The Knoevenagel condensation between benzaldehyde and ethyl cyanoacetate was further studied, lowering the catalyst loading from $10 \%$ to $2 \%$ in order to obtain information about the kinetics of the reaction. Quantitative yields of the product were achieved at about $4 \mathrm{~h}$ when using neutral base catalysts 1-3, while ionic base catalysts $4-6$ needed longer reaction time to reach full yield (Table 4.5. Fig. 4.18).

Table 4.5: Knoevenagel condensation of benzaldehyde and ethyl cyanoacetate.

\begin{tabular}{ccccccc}
\hline Catalyst & $\begin{array}{c}\text { Time } \\
(\mathrm{h})\end{array}$ & $\begin{array}{c}\text { Yield } \mathbf{A}^{\mathrm{a}} \\
(\%)\end{array}$ & $\mathrm{TON}^{\mathrm{b}}$ & $\begin{array}{c}\mathrm{TOF}^{\mathrm{c}} \\
\left(\mathrm{h}^{-1}\right)\end{array}$ & $\begin{array}{c}r_{0} \\
\left(\mathrm{~mol} \cdot \mathrm{L}^{-1} \cdot \mathrm{h}^{-1}\right)\end{array}$ & $\begin{array}{c}k \\
\left(\mathrm{~h}^{-1}\right)\end{array}$ \\
\hline $\mathbf{1}$ & 4 & 95 & 4750 & 96 & 0.49 & 1.97 \\
$\mathbf{2}$ & 4 & 93 & 4650 & 60 & 0.31 & 1.24 \\
$\mathbf{3}$ & 4 & 91 & 4550 & 56 & 0.29 & 1.15 \\
$\mathbf{4}$ & 24 & 97 & 3450 & 30 & 0.16 & 0.62 \\
$\mathbf{5}$ & 24 & 95 & 3700 & 36 & 0.19 & 0.74 \\
$\mathbf{6}$ & 24 & 93 & 4000 & 46 & 0.24 & 0.97 \\
\hline
\end{tabular}

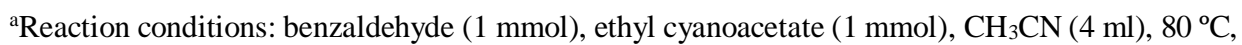
$2 \mathrm{~mol} \%$ of active site. ${ }^{\mathrm{b}} \mathrm{TON}$ calculated at $4 \mathrm{~h}$. ${ }^{\mathrm{c}} \mathrm{TOF}$ calculated at $15 \mathrm{~min}$.

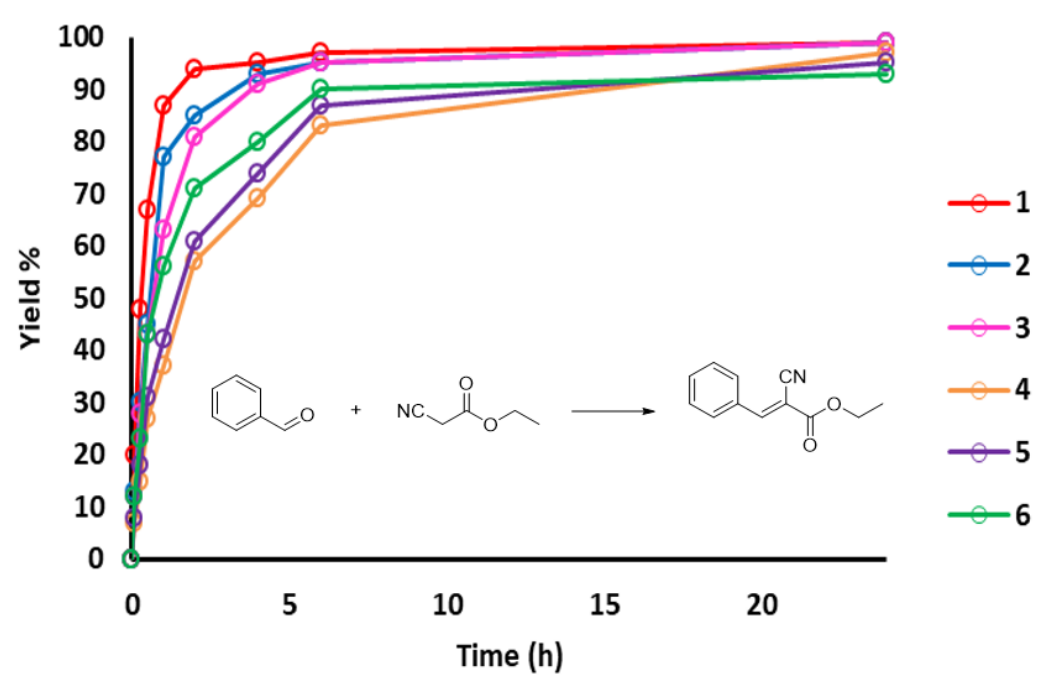

Figure 4.18: Catalytic performances of the hybrid catalysts in the Knoevenagel condensation of benzaldehyde with ethyl cyanoacetate ( 2 mol \% catalyst loading). 
Unsurprisingly, these results follow the catalytic trends observed when using a catalyst loading of $10 \%$ mol. Turnover frequencies were calculated after $15 \mathrm{~min}$. Hybrid catalyst 1 features the highest TOF, with a value comparable to reported mesoporous hybrid materials, containing proton sponges (derived from 1,8-bis(dimethylamino) naphthalene (DMAN) compounds). ${ }^{33}$ Initial rates and kinetic constants confirm the similar catalytic activity of DMAN-based organosiliceous materials ${ }^{33}$ and catalyst 1.

Deactivation and reusability of the catalysts were evaluated for the Knoevenagel condensation between benzaldehyde and ethyl cyanoacetate, Catalyst 1-3 with a catalyst loading of $2 \%$ were used in four consecutive reaction cycles. Between successive reuses, catalysts were filtered, washed with $\mathrm{CH}_{3} \mathrm{CN}$ and acetone, and dried at $100{ }^{\circ} \mathrm{C}$. The hybrid catalysts appeared to be fully recyclable, with similar yields in the first three catalytic runs and a loss in their activity in the fourth catalytic cycle (Fig. 4.19).

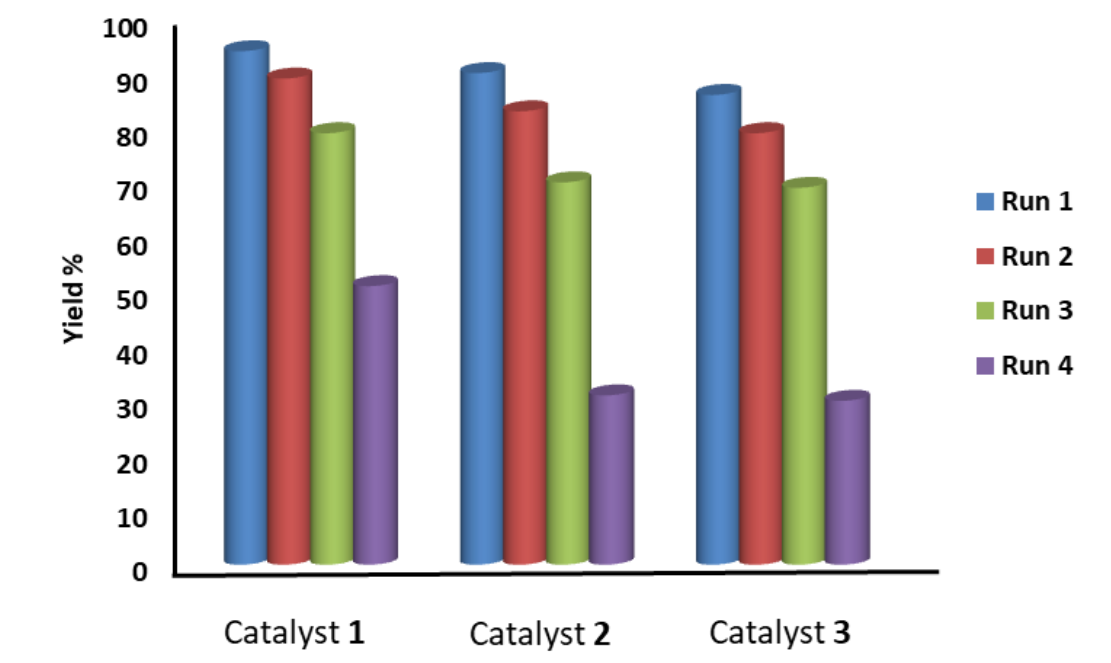

Figure 4.19: Recycling tests of Knoevenagel condensation between benzaldehyde and ethyl cyanoacetate ( 2 mol \% catalyst loading). Reaction time: $4 \mathrm{~h}$.

XRD and elemental analysis were performed on hybrid base catalysts 1-3 after four catalytic cycles, to evaluate the integrity of the organic-inorganic framework. The XRD patterns (Fig. 4.20) feature diffraction peaks typical of MCM-41 ordered structure, indicating that the framework is preserved throughout the catalytic runs. The amount of organic estimated by elemental analysis (Table. 4.6) suggests that no important leaching occurred in the first three catalytic cycles. However, after the fourth run an evident loss in the $\mathrm{N}$ content was detected, in line with the decrease observed in the obtained yield. 


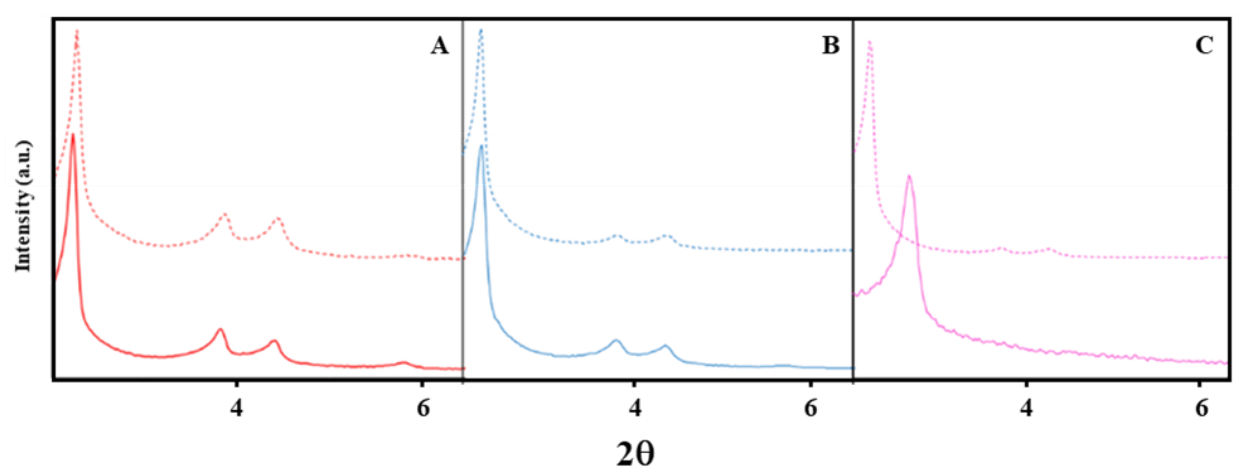

Figure 4.20: XRD patterns of hybrid base materials before (solid line) and after (dash line) four catalytic cycles. A: 1; B: 2; C: 3.

Table 4.6: Organic contents of hybrids 1-3 before and after 4 catalytic cycles.

\begin{tabular}{lllc}
\hline Catalysts & $\mathrm{N} \%$ & $\mathrm{C} \%$ & Organic content (EA)\% \\
\hline $\mathbf{1}$ & 1.09 & 3.31 & 4.40 \\
$\mathbf{1}\left(4^{\text {th }}\right.$ use $)$ & 0.87 & 7.72 & 8.59 \\
$\mathbf{2}$ & 2.36 & 5.83 & 8.19 \\
$\mathbf{2}\left(4^{\text {th }}\right.$ use $)$ & 1.85 & 9.15 & 11.00 \\
$\mathbf{3}$ & 2.52 & 7.92 & 10.44 \\
$\mathbf{3}\left(4^{\text {th }}\right.$ use $)$ & 1.92 & 9.98 & 11.90 \\
\hline
\end{tabular}

To verify that the organic moieties were actually covalently anchored onto the organic support, as opposed to just being physisorbed onto the surface, a leaching test was performed in the Knoevenagel condensation between benzaldehyde and ethyl cyanoacetate using 1-3 catalysts (10\% mol loading). For each run, after $5 \mathrm{~min}$, the reaction was stopped, the solid catalyst was filtered from the mixture and the reaction was continued in absence of the catalyst. After $2 \mathrm{~h}$, no significant increase in the yield of the final product was observed (Table 4.7), proving that no leaching occurred at the conditions at which the reaction was carried out. 
Table 4.7: Leaching tests of the hybrids $1-3(10 \% \mathrm{~mol})$ in the Knoevenagel condensation of benzaldehyde with ethyl cyanoacetate.

\begin{tabular}{ccc}
\hline Catalyst & Time (min) & Yield $(\%)$ \\
\hline $\mathbf{1}$ & 5 & 52 \\
- & 120 & 53 \\
$\mathbf{2}$ & 5 & 45 \\
- & 120 & 44 \\
$\mathbf{3}$ & 5 & 47 \\
- & 120 & 47 \\
\hline
\end{tabular}

\subsubsection{Michael addition}

The Michael addition was also used as model reaction to test the activity of the hybrid catalysts. Ethyl 2-oxocyclopentane 2-carboxylate (EtOCC) and methyl vinyl ketone (MVK) were selected as the donor and the acceptor substrate, respectively (Fig. 4.21).<smiles>CCOC(=O)C1CCCC1=O</smiles>

EtOCC<smiles>C=CC(=O)CC</smiles><smiles>CN(C)c1ccccc1</smiles>

MVK<smiles>CCOC(=O)C1(CCC(C)=O)CCCC1=O</smiles>

D

Figure 4.21: Michael addition between ethyl 2-oxocyclopentane 2-carboxylate and methyl vinyl ketone.

In all cases, no side products coming from reactions such as rearrangements, dimerizations or other condensations were observed, and high selectivity to the 1,4-adduct was achieved. The reaction between methyl vinyl ketone (MVK) and ethyl 2-oxocyclopentane 2-carboxylate (EtOCC) was carried out using hybrid catalysts 1-6 with $5 \mathrm{~mol} \%$ of catalyst loading, for $3 \mathrm{~h}$ at room temperature. In contrast with the performances observed for the Knoevenagel condensation reactions, catalysts 1-3, featuring neutral base sites, provided low yields of the final product $\mathrm{D}$ (Table 4.8). On the other hand, ionic catalysts 4-6 performed much better, with yields of product D up to values in the range of 56$69 \%$. The strong basicity provided by the hydroxide counter ion appears to be crucial to achieve high performances in Michael additions. The delocalization of the positive charge in the azolium ring of the three catalysts confers more base character to the hydroxide groups of the catalysts in the order $4<5<6$. Pyrazolium-based catalyst 4 allows for a less effective delocalization of the positive charge than imidazolium-based catalyst 5 and 6. 
Table 4.8: Michael addition between ethyl 2-oxocyclopentane 2-carboxylate and methyl vinyl ketone

\begin{tabular}{c|cc|c}
\hline \multirow{2}{*}{ Catalysts } & \multicolumn{2}{|c|}{${\text { Yield }(\%)^{\mathrm{a}}}$} & ${ }^{\mathrm{b}}$ TON \\
\hline $\mathbf{1}$ & $1 \mathrm{~h}$ & $4 \mathrm{~h}$ & 380 \\
$\mathbf{2}$ & 12 & 19 & 180 \\
$\mathbf{3}$ & 8 & 9 & 160 \\
$\mathbf{4}$ & 5 & 8 & 1120 \\
$\mathbf{5}$ & 32 & 56 & 1260 \\
$\mathbf{6}$ & 37 & 63 & 1380 \\
$\mathbf{1 - H M D S}$ & 48 & 69 & 280 \\
\hline
\end{tabular}

aReaction conditions: ethyl 2-oxocyclopentane 2-carboxylate ( $1 \mathrm{mmol})$, methyl vinyl ketone $(1 \mathrm{mmol}), \mathrm{CH}_{3} \mathrm{CN}$ $(0.5 \mathrm{ml}), 25^{\circ} \mathrm{C}, 5 \mathrm{~mol} \%$ of active site. ${ }^{\mathrm{b}} \mathrm{TON}$ calculated at $4 \mathrm{~h}$.

Additionally, catalyst 6 features an electron-donating methyl group that can stabilize the positive charge, with consequent increased base character, compared to catalyst 5 . These findings are coherent with results reported on solid base catalysts featuring hydroxide groups. ${ }^{34}$ The same reaction was performed using catalyst 1-HMDS, whose surface silanol groups have been capped with trimethyl-silyl groups. The catalytic activity decreased giving $14 \%$ yield after $4 \mathrm{~h}$, as indication that weakly acidic silanols also play an important role in Michael additions.

An enolate mechanism is proposed and depicted in Fig 4.22. Such mechanism explains the higher activity of the ionic catalysts, due to the stronger basicity conferred by the hydroxide groups. ${ }^{35}$ The catalytic cycle starts with the deprotonation of the methylenic active substrate (EtOCC) by the hydroxide group, leading to the formation of the enolate ion. Afterwards, the nucleophilic enolate reacts with the electrophilic alkene (MVK) to form a new carbon-carbon bond. Proton transfer from the protonated base to the enolate gives the final Michael addition product. Silanol groups are believed to favor substrate adsorption onto the surface of the hybrid catalysts, by means of $\mathrm{H}$-bond interactions. The adsorption of the substrates increases their local concentration, in proximity of the catalytic sites, ultimately increasing the reaction rate. Further studies would be needed to confirm this hypothesis beyond doubts. 

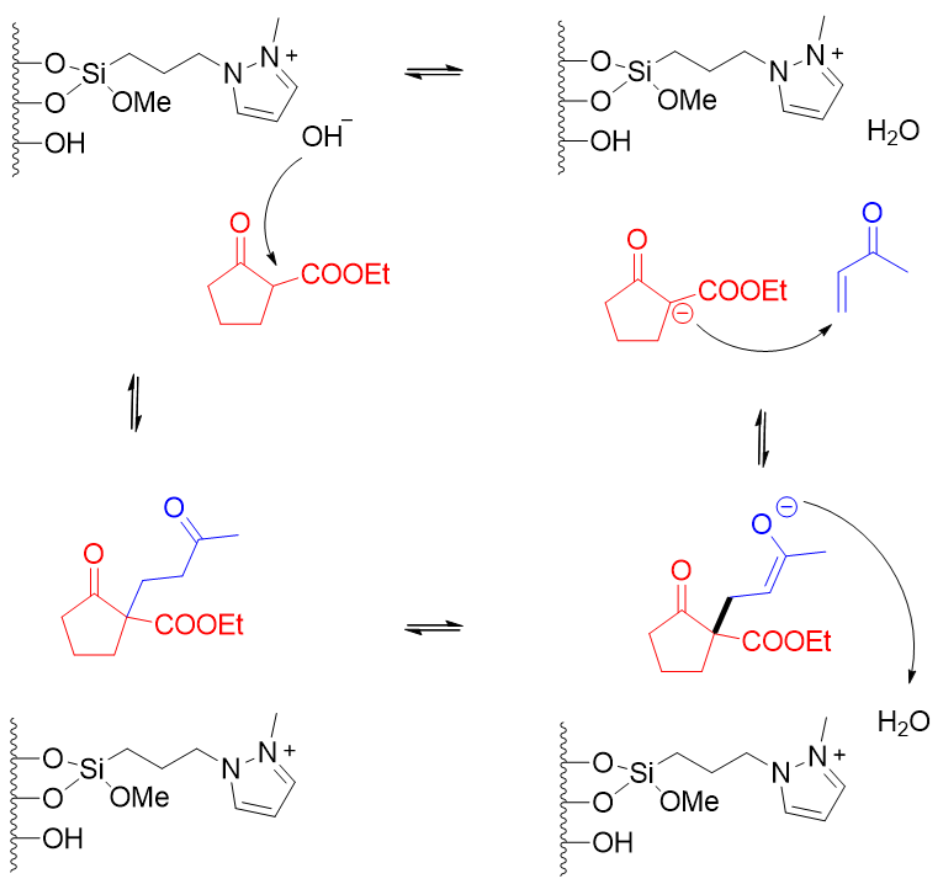

Figure 4.22: Proposed enolate catalytic cycle of Michael addition between ethyl 2-oxocyclopentane 2-carboxylate (EtOCC) and methyl vinyl ketone (MVK).

\subsubsection{Aldol condensation between furfural and MIBK}

After the Knovenagel condensations and Michael addition, the materials were tested for another aldol condensation, between furfural and methyl isobutyl ketone (MIBK).<smiles>O=Cc1ccco1</smiles>

FAL<smiles>CC(=O)CC(C)C</smiles>

MIBK
$80{ }^{\circ} \mathrm{C}, 24 \mathrm{~h}$<smiles>CC(C)CC(=O)/C=C/c1ccco1</smiles>

$\mathrm{E}$

Figure 4.23: Schematic representation of the aldol condensation between furfural (FAL) and methyl isobutyl ketone (MIBK) to give product $E$. 
Furfural is an important platform molecule originated from biomass conversion and therefore of great interest for the chemical industry. In the last decades, many efforts have been put in optimizing processes for its production ${ }^{36-38}$ and conversion to valuable chemicals and fuel components ${ }^{39,40}$. This specific reaction was selected upon the needs of the industrial partners of the MULTI2HYCAT project and it is the first step of a planned cascade reaction involving first the aldol condensation and then the hydrogenation of the newly formed olefin bond, catalyzed by noble metal nanoparticles incorporated in the hybrid catalysts as additional active site. The catalytic tests reported in this section were needed to select the best hybrid base catalyst for the aldol condensation, which was then further functionalized with Pd nanoparticles to achieve the bi-functional catalyst (see Chapter 5).

The six hybrid base catalysts and hydrophobic 1-HMDS were tested in absence of solvent (an excess of MIBK was used instead, Table 4.9). The most performing catalyst was 1, being able to achieve a conversion of $86 \%$ with a selectivity of $90 \%$ (77\% yield of product E). All other catalysts showed much lower catalytic activity with the second best catalyst, 4 , yielding only $18 \%$ of product $\mathrm{E}$. The $10 \%$ fraction of furfural that was not converted into the desired product was missing from the mass balance. It is highly likely for this to be due to a fraction of furfural polymerizing within the pore channel and for the newly formed oligomers to be hindered in their diffusion outside the mesopores. This hypothesis is supported by the results of the reuse tests, in which the catalyst was able to produce only a $25 \%$ yield of product. The catalyst was washed with DMSO between catalytic cycles. A leaching test was also performed. The reaction was monitored by liquid NMR and the catalyst was removed at $10 \%$ conversion. After the removal of the catalyst, the mixture was stirred for additional $20 \mathrm{~h}$ and no increase in furfural conversion was observed, confirming the heterogeneous nature of the catalyst.

Table 4.9: Catalytic activity of the hybrids in the condensation of furfural and MIBK

\begin{tabular}{cccc}
\hline Catalyst & Conversion $(\%)$ & Selectivity $(\%)$ & Yield $(\%)$ \\
\hline $\mathbf{1}$ & 86 & 90 & 77 \\
$\mathbf{2}$ & 26 & 46 & 12 \\
$\mathbf{3}$ & 19 & 26 & 5 \\
$\mathbf{4}$ & 33 & 58 & 19 \\
$\mathbf{5}$ & 16 & 38 & 6 \\
$\mathbf{6}$ & 14 & 79 & 11 \\
$\mathbf{1 - H M D S}$ & 6 & 33 & 2 \\
\hline
\end{tabular}

Reaction conditions: Furfural $(1 \mathrm{mmol})$, methyl isobutyl ketone $(4 \mathrm{mmol}), 80^{\circ} \mathrm{C}, 24 \mathrm{~h}, 5 \mathrm{~mol} \%$ of active site. 
A similar mechanism to that proposed for the Knoevenagel consensation is proposed for the aldol condensation between furfural and methyl isobulyl ketone (Fig. 4.24). The carbonyl oxygen of a furfural molecule undergoes $\mathrm{H}$-bond with a silanol on the silica surface. This interaction reduces the electron density on the carbonyl carbon making it more susceptible to a nucleophilic attack by the amine group. The formation of a carbinolamine and subsequent loss of a water molecule lead to the formation of an imine. A second amine group deprotonates a molecule of methyl isobutyl ketone forming an enolate species. The enolate then attacks the imine group forming a new carbon-carbon bond. Lastly, a rearrangement regenerates the catalyst, yielding the final olefin product. A mechanism in which the formation of an imine is present appears as the most plausible, considering that despite of being stronger bases, catalysts 4,5 and 6 are much less active than 1 . However, catalysts featuring secondary amine ( 2 and 3 ) also showed poor catalytic activity in this reaction. More studies would be needed to explain these findings. Notably, the ionic/non-ionic character of a catalyst does not seem to be a key factor to forecast the activity of a given catalyst. The catalytic activity of catalyst 1 drops dramatically when silanols are capped with trimethyl-silyl residues (catalyst 1-HMDS), as confirmation of the crucial role played by silanol groups in activating the carbonyl group.

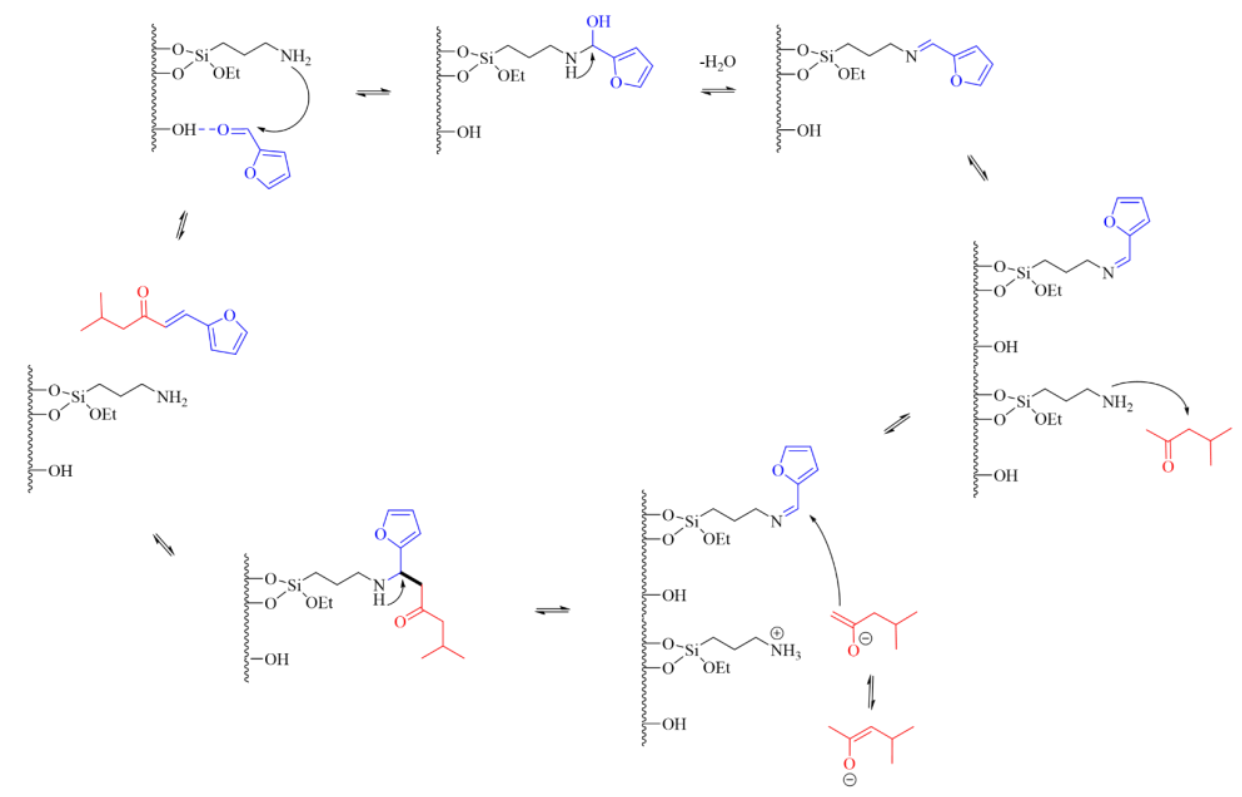

Figure 4.24: Proposed mechanism for the aldol condensation between furfural and methyl isobutyl ketone. 


\subsection{Synthesis of silica-supported phosphazene materials (P1)}

Phosphazene are strong non-metallic non-ionic and low-nucleophilic bases, with a $\mathrm{pKa}$ which varies depending on the number of $\mathrm{P}=\mathrm{N}$ units (Fig. 4.25). ${ }^{41}$ Throughout the years, many groups have explored the potential of phosphazenes as catalysts (or as co-catalysts) in different reactions such as the polyaddition of a five-membered dicyclic carbonate with a poly(ethylene glycol)-based diamine, ${ }^{42}$ metal-free living copolymerization of carbonyl sulfide with epoxides ${ }^{43}$ or ring-opening polymerizations. ${ }^{44,45}$

P1

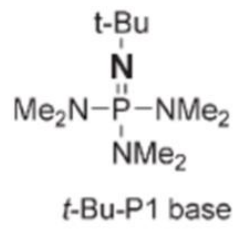

P2

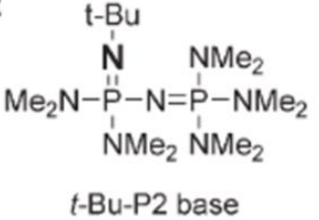

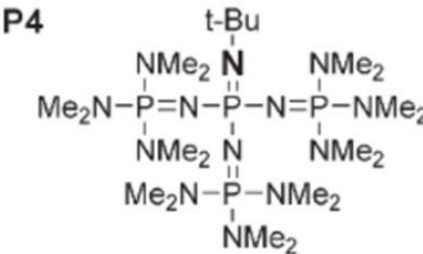

$t$-Bu-P4 base

Figure 4.25: Schematic representation of some of the most commonly used phosphazene bases. Adapted from ref. 46.

P1-type phosphazenes are the simplest, carrying only one iminophosphorane moiety. Nevertheless, their high catalytic activity has been reported in multiple studies involving base demanding reactions. Examples include the aza-Henry reaction of ketimines, ${ }^{47}$ ringopening alternating copolymerization of phthalic anhydride with different epoxides, ${ }^{48}$ regioselective esterifications of glycerol derivatives, ${ }^{49}$ synthesis of 1-methoxycarbonyl pyrrole $^{50}$ and water free biodiesel productions. ${ }^{49}$ The methodologies for preparation of phosphazenes suffers from drawbacks such as the time-consuming preparation of the reactive intermediates, the necessity of their isolation, the use and the formation of irritating gases. ${ }^{51}$ Given the complexity of the synthesis, only few examples of supported phosphazenes have been up to now reported in the literature for applications as heterogeneous catalyst. ${ }^{52-54}$

In this Section, an easy and reproducible methodology to achieve heterogeneous $\mathrm{P}^{+} \mathrm{OH}^{-}$ moieties supported over MCM-41 type silica is reported (P1-MCM-41, Fig. 4.26 A). Silyl-derivative 3P was obtained by reacting (3-azidopropyl)(triethoxy)silane with hexamethylphosphorous triamide. ${ }^{52}{ }^{31} \mathrm{P}$ NMR spectrum of the $3 \mathrm{P}$ was recorded in toluene (Fig. $4.26 \mathrm{C}$ ). An intense signal consistent with the presence of phosphazene moieties is observed at $24 \mathrm{ppm}$. However, when the spectrum was recorded using water as solvent, such signal ceases to be observed. Instead, a single signal centered at $40 \mathrm{ppm}$ is observed and ascribed to the phosphazenium ion. ${ }^{13} \mathrm{C}$ NMR signals are also consistent with the presence of compound 3P (Fig. 3.26 B). 


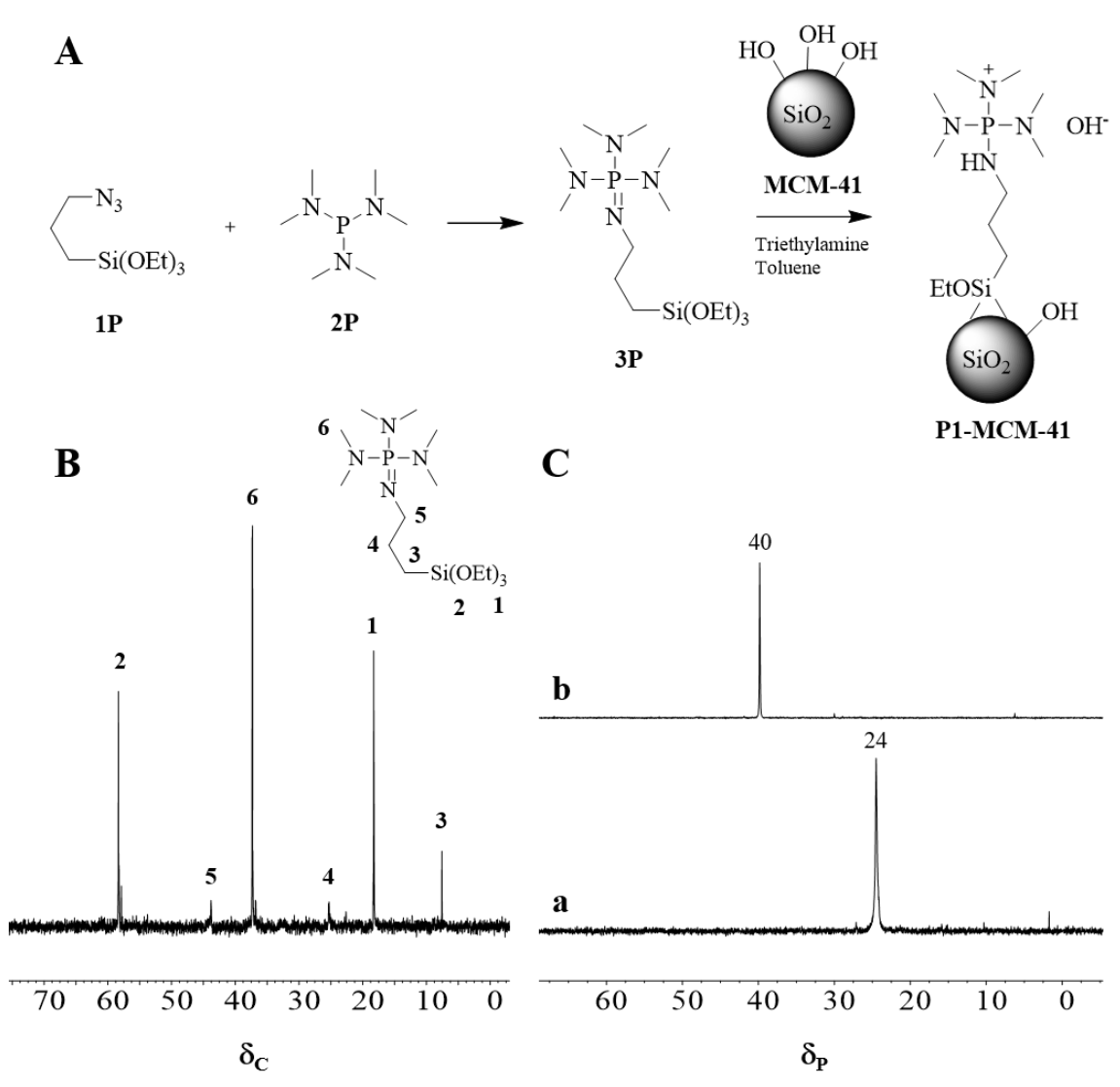

Figure 4.26 A: Schematic representation of the synthesis of P1-MCM-41. B: ${ }^{13} \mathrm{C}$ liquid NMR spectrum of silyl-derivative 3P. C: ${ }^{31} \mathrm{P}$ liquid NMR spectrum of $3 \mathrm{P}$ in toluene-d6 (a) and $\mathrm{D}_{2} \mathrm{O}$ (b).

Silyl-derivative 3P was grafted onto MCM-41. Due to the strong basicity of the P1 moieties, it was expected that during the grafting procedure phosphazene moieties would hydrolyze to phosphazenium hydroxide species. To ensure the presence of hydroxyl as counter-ion balancing the pending phosphazenium, the solid was exchanged twice with a $0.2 \mathrm{M}$ solution of tetramethyammonium hydroxide pentahydrate in methanol.

A multi-technique approach was used to evaluate the integrity of the support after the grafting procedure. X-ray diffractogram was recorded to probe the structural properties of P1-MCM-41 (Fig. 4.27 A). The typical diffraction band of the MCM-41-type materi- 
als is observed at $2.42 \theta$ degrees and bestowed to the (100) planes. Signals of weak intensity can also be seen between 4 and $52 \theta$ degrees and are ascribed to the (110) and (200) planes, typical of the hexagonal mesopore framework of MCM-41.

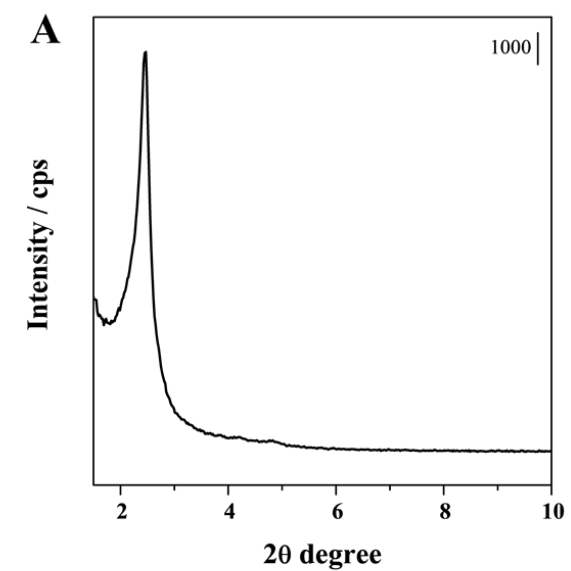

C

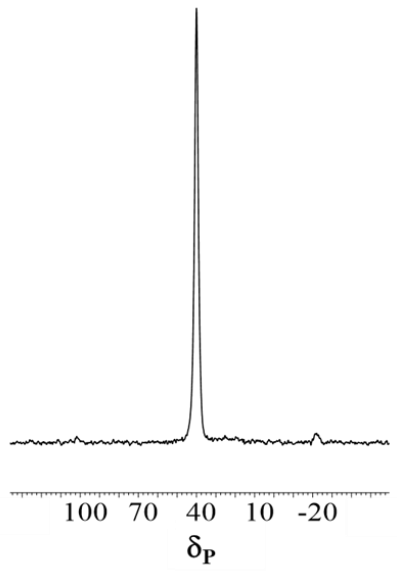

D

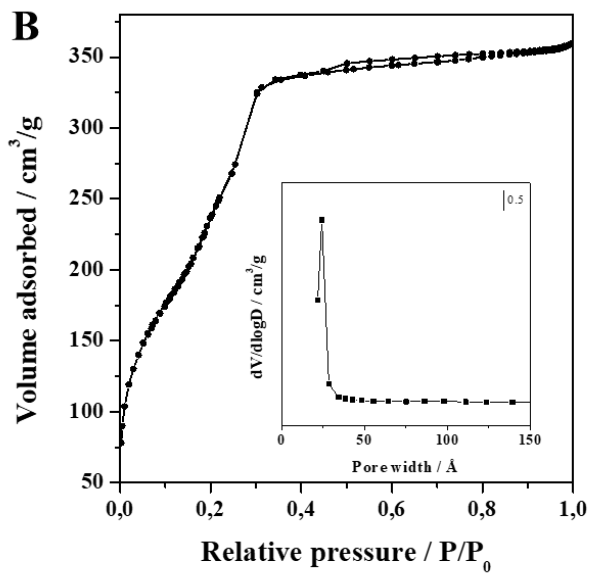

$\mathbf{E}$

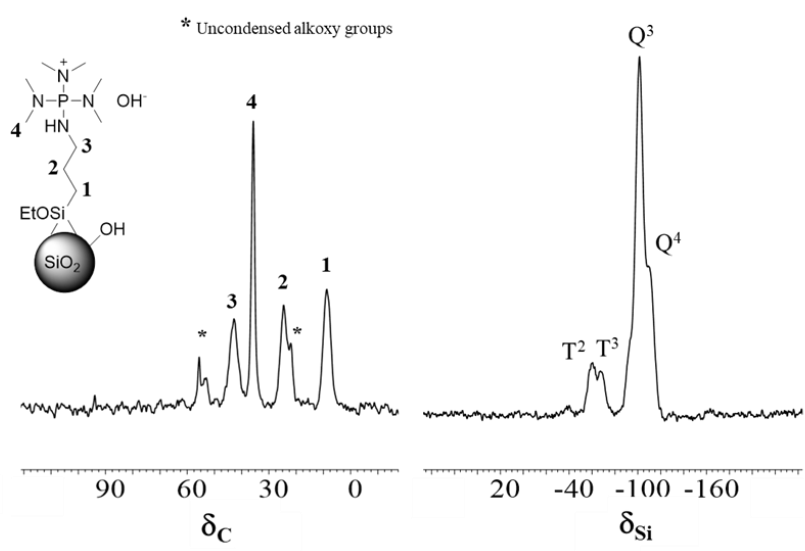

Figure 4.27 A: XRD pattern of P1-MCM-41. B: $\mathrm{N}_{2}$ physisorption isotherm $(77 \mathrm{~K})$ recorded on P1-MCM-41. Inset: BJH pore size distribution of P1-MCM-41. C: ${ }^{31} \mathrm{P}$ solid state MAS NMR spectrum of P1-MCM-41. D: ${ }^{29}$ Si solid state CP/MAS NMR spectrum of P1-MCM-41. E: ${ }^{13} \mathrm{C}$ solid state CP/MAS NMR spectrum of P1-MCM-41. 
The textural properties of the hybrid material were studied by means of $\mathrm{N}_{2}$ physisorption analysis at $77 \mathrm{~K}$ (Fig. $4.27 \mathrm{~B}$ ). The isotherm shows a type IV behavior, with a hysteresis loop, typical of mesoporous materials. The $\mathrm{N}_{2}$ uptake is consistent with MCM-41-type solids. By analyzing the isotherm with the Brunauer-Emmett-Teller (BET) method a surface area of $970 \mathrm{~m}^{2} / \mathrm{g}$ was observed, while the Barrett-Joyner-Halenda (BJH) analysis showed a pore size distribution centered at about $20 \AA$ (Fig. 4.27 B, inset).

Focusing on the pendant organic moieties, the grafting procedure does not compromise the integrity of the phosphazene residues. ${ }^{31} \mathrm{P}$ solid state MAS NMR of P1-MCM-41 show a single sharp signal centered at about $40 \mathrm{ppm}$, related to the phosphazenium species anchored onto the surface of the support (Fig. $4.27 \mathrm{C}$ ). ${ }^{13} \mathrm{C}$ solid state MAS NMR spectrum provides additional proof of the preservation of the grafted P1 moieties, with all the chemical shifts observed in the spectrum recorded on silyl-derivative 3P preserved after the grafting procedure (Fig. 4.27 D). Finally, ${ }^{29} \mathrm{Si}$ solid state CP MAS NMR spectrum provides information on the silicon speciation in the hybrid material (Fig. 4.27 E). Firstly, the presence of the support gives rise to the $\mathrm{Q}^{4}$ and $\mathrm{Q}^{3}$ signals, related to bulk and surface silicon atom, respectively. Additionally, the signals $\mathrm{T}^{3}$ and $\mathrm{T}^{2}$ are observed, due to silicon atoms directly bounded to carbon atoms, as confirmation of the incorporation of silyl-derivative 3 within the mesoporous network. ${ }^{29} \mathrm{Si}$ solid state BD MAS NMR spectrum was also recorded on the samples. The integrated area of the T peaks (i.e. silicon atoms bounded to carbon atoms, coming from the silyl-derivative) corresponded to $4 \%$ of the integrated area of all peaks $(T+Q)$.

Besides the potential catalytic application of this hybrid material, not explored in this Thesis, this work opens up to the potential use of $3 \mathrm{P}$ for the synthesis of more complex and novel hybrid catalysts. Generally, silyl-derivatives featuring alkoxy groups can show low stability, with the tendency of undergoing spontaneous hydrolysis/condensation, especially when featuring strongly acidic or basic functional groups. However, 3P appeared to be relatively stable, which makes it a possible candidate for use as building block in the synthesis of mono- and multi-functional hybrid materials. 


\subsection{Conclusions}

The work reported in this Chapter focused on the development of silica-supported organocatalysts featuring base active sites.

- All hybrids were prepared by grafting silyl-derivatives onto MCM-41 support. Such silyl-derivatives are substrates featuring the base active site and a highly reactive trialkoxysilyl group. Two of them, namely aminopropyl triethoxysilane (I) and N(2-aminoethyl)-3-(trimethoxysilyl)propylamine (II), were bought from commercial sources, while other four were synthesized starting from commercially available precursors. Compound III was prepared by reacting 2-aminopyrrolidine and 3-isocyanatopropyl triethoxysilane at $-57{ }^{\circ} \mathrm{C}$, to avoid the reaction between the isocyanate group and the secondary amine. Compounds IV, V and VI were prepared by reacting 3-iodopropyltrimethoxysilane and 1-methylpyrazole, 1-methylimidazol and 1,2-dimethylimidazol, respectively. Six hybrid catalysts were obtained and named 1-6. To achieve the catalytically active form, i.e. the one featuring hydroxyl species as counter ions, ionic hybrids IV-VI were exchanged three times in a $0.2 \mathrm{M}$ tetramethylammonium hydroxide solution in methanol. Silica supports bare surface silanol groups on their surface that are reportedly beneficial for the cooperative catalytic activity of supported base catalyst in a number of reactions. In fact, they can act in a synergistic fashion, interacting with substrates through $\mathrm{H}$-bond and making them more susceptible to nucleophilic attack by the base. Such cooperative catalysis has been thoroughly studied and the many articles reported in the scientific literature, allowed for the optimal selection of the alkyl linker: A propyl chain was used, as it was reported to be the optimal length for promoting cooperative catalysis in MCM41-based hybrids. A passivated version of catalyst 1 was also prepared by reacting the surface silanol with hexamethyl disilazane (HMDS), in order to directly evaluate the effect of surface silanols on the catalytic activity of the hybrids.

- The catalysts were thoroughly characterized by means of a multi-technique approach. X-ray powder diffraction confirmed the integrity of the ordered morphology throughout grafting and ion exchange in alkaline $\mathrm{pH}$. Thermogravimetric analyses provided useful information on the thermal stability of the hybrid catalysts and specifically of the pendant organic moieties. Elemental analyses indicated that approximately the same molar amount of silyl-derivative was grafted onto MCM-41, for each of the six hybrid materials. FTIR, ${ }^{13} \mathrm{C}$ and ${ }^{29} \mathrm{Si}$ NMR spectroscopies confirmed the integrity of the organic species throughout the grafting, confirming that the target hybrid base catalysts were achieved. $\mathrm{N}_{2}$ physisoption analyses at $77 \mathrm{~K}$ indicated that the six hybrid materials featured textural properties consistent with mesoporous solids derived from MCM-41 support.

- The hybrid catalysts were tested in multiple reaction requiring a base catalyst. Firstly, Knovenagel condensation reactions of benzaldehyde and substrates featuring different $\mathrm{pKa}$ were performed. The selected substrates were ethyl cyanoacetate 
( $\mathrm{pKa} \approx 9$ ), ethyl acetoacetate $(\mathrm{pKa} \approx 11)$ and diethyl malonate $(\mathrm{pKa} \approx 13)$. Non-ionic catalysts 1-3 showed higher catalytic activity, compared to ionic catalysts 4-6. In particular, catalyst 1 , featuring propyl amine moieties, was the most performing, with a TOF value of $96 \mathrm{~h}^{-1}$ for the reaction between benzaldehyde and ethyl cyanoacetate. The silanol-capped version of 1 (1-HMDS) performed much worse than its hydrophilic counterpart, highlighting the key role played by surface silanol in cooperatively catalyzing the reaction. An imine mechanism was proposed for this reaction. Recycle tests were carried out, indicating stable catalytic activity over three catalytic cycles.

- The catalytic activity of the hybrid catalysts was evaluated in the Michael addition of ethyl 2-oxocyclopentane 2-carboxylate (EtOCC) and methyl vinyl ketone (MVK). Contrarily to what observed in the Knoevenagel condensation reaction, ionic catalysts 4-6 featured much higher activity than non-ionic catalysts 1-3. An enolate mechanism was proposed for this reaction. The activity of the catalyst is proportional to ease with which the hydroxyl group can react and therefore proportional to the ability that the cation has in stabilizing the positive charge, while its counter ion is involved in the catalytic cycle. Accordingly, the positive charge is delocalized over a smaller area in catalyst 4 than in 5 and 6 and therefore the former shows lower catalytic activity. The slightly higher activity of catalyst 6 was explained by considering the electron-donating effect of the methyl substituent on the carbon in position 2 of the azo-ring. For this reaction, also hydrophobic 1-HMDS performed worse than its hydrophilic counterpart.

- Lastly, the hybrid catalysts were tested in the reaction between furfural and methyl isobutyl ketone. This reaction was selected upon the need of the industrial partners of the MULTI2HYCAT project and represents the first step of a tandem reaction, featuring as second step the hydrogenation of the newly formed C-C double bond. Catalyst 1 showed the highest catalytic activity with $77 \%$ yield of the desired product after $24 \mathrm{~h}$. The other five catalysts featured a yield after $24 \mathrm{~h}$ below 20\%. An imine mechanism was proposed for this reaction. Catalyst 1 was chosen to be further functionalized with Pd nanoparticles. This part of the work will be described in Chapter 5.

- In the last section of the Chapter, a versatile strategy for synthesizing silica-supported P1 phosphazenium hydroxide moieties was detailed. A silyl-derivative featuring P1 moieties was developed in a one-step synthesis and grafted onto MCM-41 support and exchanged twice with a $0.2 \mathrm{M}$ solution of tetramethylammonium hydroxide in methanol. X-ray powder diffraction of the hybrid showed the characteristic diffraction pattern of MCM-41-like materials; $\mathrm{N}_{2}$ physisoption analysis also indicated the preservation of the mesoporous channels typical of this support; ${ }^{31} \mathrm{P},{ }^{13} \mathrm{C}$ and ${ }^{29} \mathrm{Si}$ solid state MAS NMR showed chemical shifts consistent with the presence of the supported moieties that were the target of the synthesis. This work is intended to be a proof of concept, as the silyl-derivative featuring the phosphazene group is 
Hybrid Mesoporous Silica-Supported Base Organocatalysts for C-C Bond Formation

relatively stable and can be used for the future design of mono- or multi-functional effective and active catalysts. 


\subsection{References}

(1) Denmark, S. E.; Beutner, G. L. Angew. Chem. Int. Ed. 2008, 47, 1560 - 1638.

(2) Hattori, H.; Tanaka, Y.; Tanabe, K. Chem. Lett. 1975, 4, 659-660.

(3) Liu, X.; He, H.; Wang, Y.; Zhu, S. Catal. Commun. 2007, 8, 1107-1111.

(4) Iizuka, T.; Hattori, H.; Ohno, Y.; Sohma, J.; Tanabe, K. J. Catal. 1971, 22, 130-139.

(5) Ueda, W.; Kuwabara, T.; Ohshida, T.; Morikawa, Y. J. Chem. Soc. Chem. Commun. 1990, 22, 1558-1559.

(6) Malinowski, S.; Szczepańska, S.; Bielański, A.; Sloczyński, J. J. Catal. 1965, 4, 324 331.

(7) Zhang, G.; Hattori, H.; Tanabe, K. Appl. Catal. 1988, 36, 189-197.

(8) Martra, G.; Cacciatori, T.; Marchese, L.; Hargreaves, J. S. J.; Mellor, I. M.; Joyner, R. W.; Coluccia, S. Catal. Today 2001, 70, 121-130.

(9) Konuray, A. O.; Fernández-Francos, X.; Ramis, X. Polym. Chem. 2017, 8, 59345947.

(10) Borch Jacobsen, C.; Meldal, M.; Diness, F. Chem. - A Eur. J. 2017, 23, 846-851.

(11) Khoi, van N.; Morrill, L. C.; Smith, A. D.; Romo, D. Lewis Base Catal. Org. Synth. 2016, 2, 527-653.

(12) Brunelli, N. A.; Didas, S. A.; Venkatasubbaiah, K.; Jones, C. W. J. Am. Chem. Soc. 2012, 134, 13950-13953.

(13) Lauwaert, J.; Moschetta, E. G.; Van Der Voort, P.; Thybaut, J. W.; Jones, C. W.; Marin, G. B. J. Catal. 2015, 325, 19-25.

(14) Lauwaert, J.; De Canck, E.; Esquivel, D.; Van Der Voort, P.; Thybaut, J. W.; Marin, G. B. Catal. Today 2015, 246, 35-45.

(15) Kandel, K.; Althaus, S. M.; Peeraphatdit, C.; Kobayashi, T.; Trewyn, B. G.; Pruski, M.; Slowing, I. I. J. Catal. 2012, 291, 63-68.

(16) Brunelli, N. A.; Jones, C. W. J. Catal. 2013, 308, 60-72.

(17) Brunelli, N. A.; Venkatasubbaiah, K.; Jones, C. W. Chem. Mater. 2012, 24, 2433 2442.

(18) Collier, V. E.; Ellebracht, N. C.; Lindy, G. I.; Moschetta, E. G.; Jones, C. W. ACS Catal. 2016, 6, 460-468.

(19) Kim, K. C.; Moschetta, E. G.; Jones, C. W.; Jang, S. S. J. Am. Chem. Soc. 2016, 138, 7664-7672.

(20) Erigoni, A.; Hernández-Soto, M. C.; Rey, F.; Segarra, C.; Díaz, U. Catal. Today 2020, 345, 227-236. 
(21) Tanev, P. T.; Pinnavaia, T. J. Science 1995, 267, 865-867.

(22) Kawahara, K.; Hagiwara, Y.; Shimojima, A.; Kuroda, K. J. Mater. Chem. 2008, 18, 3193-3195.

(23) Socrates, G. Infrared Characteristics Group Frequencies, 1980, Wiley.

(24) Haddad, B.; Mokhtar, D.; Goussem, M.; Belarbi, E. habib; Villemin, D.; Bresson, S.; Rahmouni, M.; Dhumal, N. R.; Kim, H. J.; Kiefer, J. J. Mol. Struct. 2017, 1134, 582590.

(25) Rodriguez, I.; Iborra, S.; Corma, A.; Rey, F.; Jordá, J. L. Chem. Commun. 1999, 7, 593-594.

(26) Climent, M. J.; Corma, A.; Iborra, S.; Epping, K.; Velty, A. J. Catal. 2004, 225, 316326.

(27) Prout, F. S.; Beaucaire, V. D.; Dyrkacz, G. R.; Koppes, W. M.; Kuznicki, R. E.; Marlewski, T. A.; Pienkowski, J. J.; Puda, J. M. J. Org. Chem. 1973, 38, 1512-1517.

(28) Jones, G. C., Knoevenagle Condensation. Organic Reactions. 1964, Wiley.

(29) Gaona, A.; Moreno, J. M.; Velty, A.; Díaz, U.; Corma, A. J. Mater. Chem. A 2014, 2, 19360-19375.

(30) Gianotti, E.; Diaz, U.; Velty, A.; Corma, A. Eur. J. Inorg. Chem. 2012, 32, 51755185.

(31) Xu, L.; Wang, C.; Guan, J. J. Solid State Chem. 2014, 213, 250-255.

(32) Climent, M. J.; Corma, A.; Iborra, S.; Velty, A. J. Mol. Catal. A Chem. 2002, 182$183,327-342$.

(33) Corma, A.; Iborra, S.; Rodríguez, I.; Sánchez, F. J. Catal. 2002, 211, 208-215.

(34) Rodriguez, I.; Iborra, S.; Rey, F.; Corma, A. Appl. Catal. A Gen. 2000, 194, 241252.

(35) Wang, X.-W.; Wang, Y.; Jia, J.; Chen, S.; Salo, E. C.; Kerrigan, N. J.; Furuta, T.; Kawabata, T.; Smith, A. D.; Woods, P. A.; Suzuki, K.; Takikawa, H.; DiRocco, D. A.; Rovis, T.; Chiang, P.-C.; Bode, J. W.; Hatakeyama, S.; Fan, Y. C.; Kwon, O.; Yliniemelä-Sipari, S. M.; Piisola, A.; Pihko, P. M.; Benohoud, M.; Hayashi, Y.; Mase, N.; Mukherjee, S.; MacMillan, D. W. C.; Beeson, T. D.; Watson, A. J. B.; Liu, Y.; Melchiorre, P.; Christmann, M.; Wong, O. A.; Ramirez, T. A.; Shi, Y.; GarcíaGarcía, P.; Mase, N. Lewis Base and Acid Catalysts. 2012, Wiley.

(36) Luo, Y.; Li, Z.; Li, X.; Liu, X.; Fan, J.; Clark, J. H.; Hu, C. Catal. Today 2019, 319, $14-24$.

(37) Agirrezabal-Telleria, I.; Gandarias, I.; Arias, P. L. Catal. Today 2014, 234, 42-58.

(38) Peleteiro, S.; Rivas, S.; Alonso, J. L.; Santos, V.; Parajó, J. C. Bioresour. Technol. 2016, 202, 181-191. 
(39) Yan, K.; Wu, G.; Lafleur, T.; Jarvis, C. Renew. Sustain. Energy Rev. 2014, 38, 663 676.

(40) Li, X.; Jia, P.; Wang, T. ACS Catal. 2016, 6, 7621-7640.

(41) Schwesinger, R.; Schlemper, H. Angew. Chemie Int. Ed. English 1987, 26, $1167-$ 1169.

(42) Bossion, A.; Aguirresarobe, R. H.; Irusta, L.; Taton, D.; Cramail, H.; Grau, E.; Mecerreyes, D.; Su, C.; Liu, G.; Müller, A. J.; Sardon, H. Macromolecules 2018, 51, $5556-5566$.

(43) Zhang, C. J.; Wu, H. L.; Li, Y.; Yang, J. L.; Zhang, X. H. Nat. Commun. 2018, 9, 1 10.

(44) Liu, S.; Li, H.; Zhao, N.; Li, Z. ACS Macro Lett. 2018, 7, 624-628.

(45) Fuchise, K.; Igarashi, M.; Sato, K.; Shimada, S. Chem. Sci. 2018, 9, 2879-2891.

(46) Suzawa, K.; Ueno, M.; Wheatley, A. E. H.; Kondo, Y. Chem. Commun. 2006, 46, 4850-4852.

(47) Pahadi, N. K.; Ube, H.; Terada, M. Tetrahedron Lett. 2007, 48, 8700-8703.

(48) Li, H.; Luo, H.; Zhao, J.; Zhang, G. Macromolecules 2018, 51, 2247-2257.

(49) Cerro-Alarcón, M.; Corma, A.; Iborra, S.; Gómez, J. P. Appl. Catal. A Gen. 2008, 346, 52-57.

(50) Quaranta, E.; Carafa, M.; Trani, F. Appl. Catal. B Environ. 2009, 91, 380-388.

(51) Alexandrova, A. V.; Mašek, T.; Polyakova, S. M.; Císařová, I.; Saame, J.; Leito, I.; Lyapkalo, I. M. European J. Org. Chem. 2013, 9, 1811-1823.

(52) Liras, M.; Verde-Sesto, E.; Iglesias, M.; Sánchez, F. Eur. Polym. J. 2017, 95, 775 784.

(53) Kim, K. S.; Kim, J. H.; Seo, G. Chem. Commun. 2003, 3, 372-373.

(54) Kim, M.-Y.; Seo, G.; Kwon, Z.; Chang, D. R. Chem. Commun 2009, 3110-3112. 

Chapter 5

\section{Synthesis of Multi-Functional Hybrid Catalysts for Cascade Reactions}

Different multi-functional hybrid catalysts featuring a base and a metal site were prepared through different synthetic approaches. The catalysts underwent preliminary characterization and catalytic tests, which acted as proofof-concept of the validity of the synthetic approaches and paved the way for further studies.

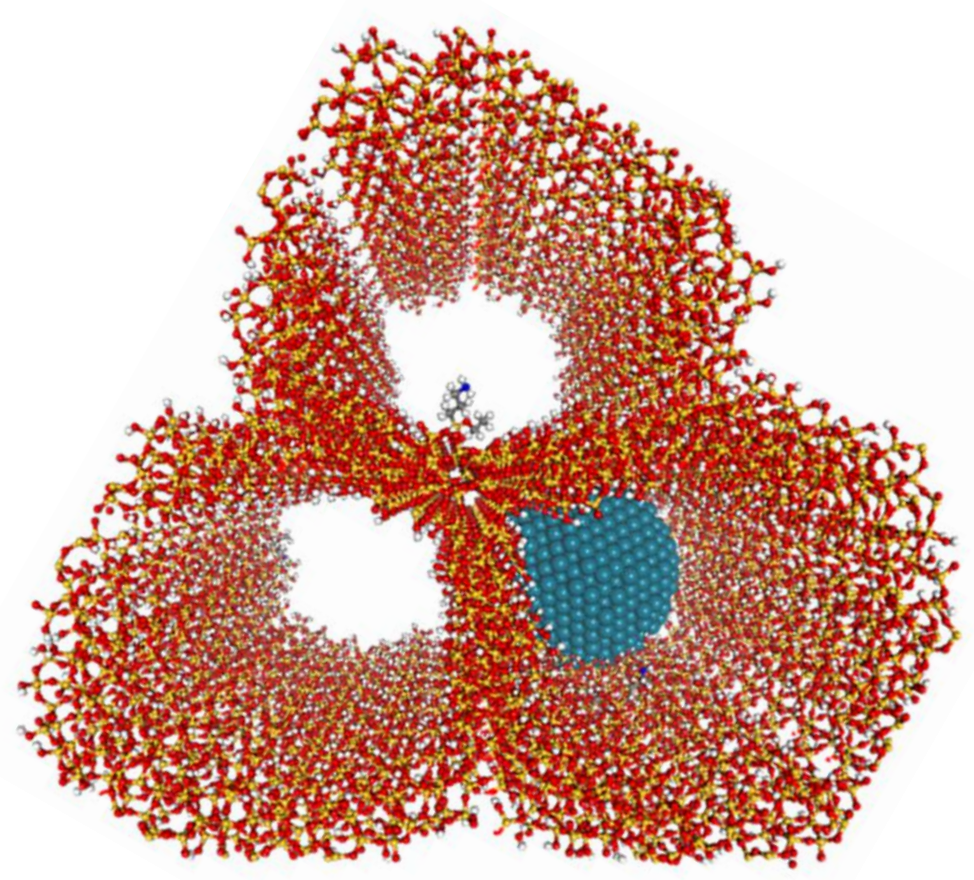



Supported metal catalysts based on metal crystallites close to the nanometer size have been prepared and used AS heterogeneous catalysts for more than 60 years. By using the characterization techniques available at the time (mainly transmission electron microscopy and chemisorption of gases), scientists have been able to classify reactions on metal catalysts as structure-sensitive and non-structure-sensitive. ${ }^{1}$ Today, we know that progressive reduction of particle size, from nanoparticles (NPs) to clusters and eventually to single atoms alters the electronic structure which, in turn, produces changes in the catalytic properties of the materials (Fig. 5.1). However, while a very large amount of experimental work and knowledge has been gathered along the years, a unified theory able to explain and predict the behavior of various metal catalysts with different particle sizes for different reactions has not been produced, yet. $^{2}$

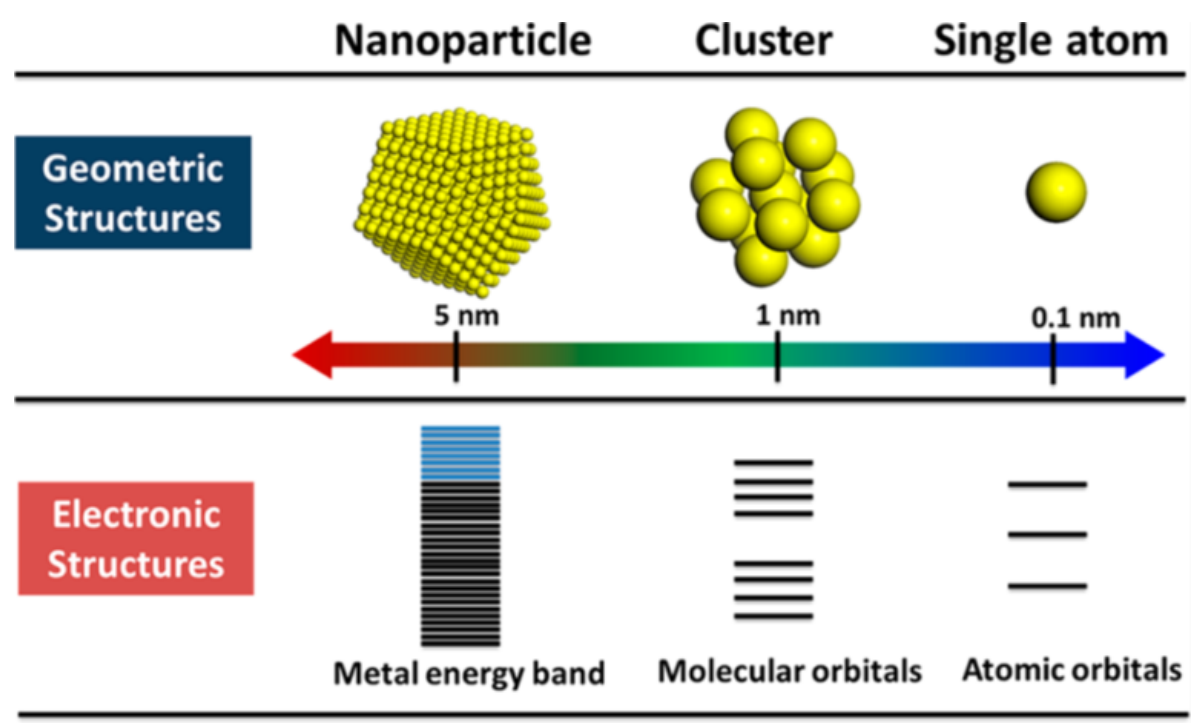

Figure 5.1: Geometric and electronic structures of single atoms, clusters, and nanoparticles. ${ }^{2}$

In the context of heterogeneous catalysis, palladium is surely one of the most interesting transition elements. Many Pd-catalyzed reactions have been developed over the past four decades, with many studies published on the topic and industrial implementations of Pdbased catalysts. Such interest is due to two main reasons. Firstly, the electronic structure of Pd makes it optimal for catalyzing $\mathrm{C}-\mathrm{C}$ and $\mathrm{C}-\mathrm{N}$ bond formation under sustainable conditions and with excellent yields. The mild conditions needed are tolerable by many functional groups, which allow reacting complex molecules, the prime example being drug precursors. ${ }^{3}$ Suzuki, Heck, Sonogashira, Buchwald-Hartwig and Stille are the most important reactions catalyzed by Pd, usually under homogeneous conditions. However, quantitative separation of the metal catalyst from the reaction mixture is a crucial aspect 
of the process, due to their cost and, more importantly, because metal contamination is highly regulated by the pharmaceutical industry, possibly the most important branch of the chemical industry in which these catalysts are implemented. Immobilization of palladium onto several solid supports have been reported by many authors, through different approaches, leading to very different materials. ${ }^{4-6}$ Considering the vast amount of research reported on the topic, with the scope of narrowing the focus to studies that are related to the work reported in this Chapter, relevant examples from the state of the art of silica-supported palladium nanoparticle and of some silica-supported palladium complexes are described below. Studies involving the incorporation of multiple active sites for cascade reactions will receive particular attention, considering the context of this Doctoral Thesis work. 


\subsection{Silica-supported palladium nanoparticles}

Palladium nanoparticles feature high surface energy, which contributes to making them so active in catalyzing demanding reactions. However, such high surface energy also translates in a strong tendency to aggregation and consequent formation of Pd black, whenever they are used under homogeneous conditions. ${ }^{7}$ To avoid this, confinement of Pd nanoparticles within nanoreactors, such as cavities or channels of solid supports, has been carried out by several authors by means of different synthetic strategies. ${ }^{8}$ Several porous materials have been used as nanoreactors such as zeolites, ${ }^{9}$ polymers, ${ }^{10}$ carbon nanotubes, ${ }^{11}$ metal-organic frameworks ${ }^{12}$ and mesoporous silica-based materials, to this scope. $^{13}$

Among these supports, mesoporous silicas stand out due to their high thermal and chemical stability, slow-cost and facile synthesis and the ease of recovery and reuse by simple centrifugation or filtration. Moreover, their high surface area, tunable pore size and narrow pore size distribution provide key advantages for their scope. ${ }^{14}$ To this point, Barau et al. reported on the synthesis of silica-supported palladium nanoparticles, achieved through different approaches. Firstly, they prepared a catalyst by adding the palladium precursor in the synthetic mixture during the preparation of the mesoporous support (cogelation); then, they prepared two more catalysts by wet impregnation using a Pd-containing solution and either a pure silica support or a mesoporous silica previously functionalized with amino-propyl groups. The catalyst prepared by wet impregnation using an amino-propyl-functionalized support showed superior catalytic activity in the Heck reaction between iodobenzene and methylacrylate. The authors reported that by preparing the catalyst in such manner, a homogeneously distributed nanoparticle system, stabilized by the pending organic moieties, should be achieved. ${ }^{15}$

In another study, Li et al. prepared a series of catalysts by immobilizing Pd nanoparticles onto amino-functionalized SBA-15. Firstly, the hybrid support was prepared by one-pot co-condensation of TEOS and aminopropyl trimethoxysilane (APTMS) in presence of block copolymer P123; then, palladium nanoparticles were supported by adsorption/reduction procedure. The amino groups present on the silica support were designed for playing two roles: stabilizing the Pd NPs against aggregation and leaching, both by weak interaction between the $\mathrm{N}$ lone pair and the empty orbitals of the Pd atoms and by partial occlusion of the pore channels, and acting as Brønsted base sites in a cascade reaction (Fig. 5.2). The bi-functional catalyst provided full yield of the hydrogenated product. To access the key role played by each of the two active sites, mono-functional catalysts featuring only one of the two sites were prepared. As expected, $\mathrm{NH}_{2}$-functionalized SBA-15 led to a $100 \%$ yield of product B1 (Fig. 5.2 B) and no conversion was observed using Pd NPs supported onto pure silica SBA-15. Interestingly, a physical mixture of the two mono-functional catalysts also gave a $100 \%$ yield of the desired product $\mathrm{C} 1$. 
A

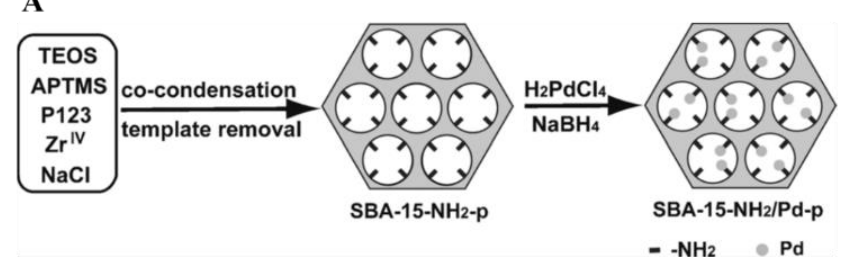

B
C

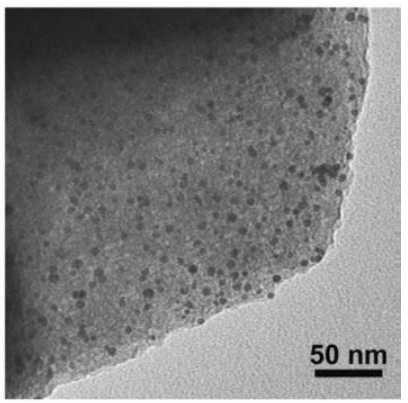

Figure 5.2 A: Synthesis procedure for the SBA-15- $\mathrm{NH}_{2} / \mathrm{Pd}-\mathrm{p}$ catalyst. B: One-pot Knoevenagel condensation/hydrogenation multi-step cascade reaction sequence between benzaldehyde and ethyl cyanoacetate. $\mathrm{C}$ : TEM image of the $\mathrm{SBA}-15-\mathrm{NH}_{2} / \mathrm{Pd}$-p catalyst after five catalytic cycles. Adapted from ref. 16.

However, while the bi-functional catalyst was able to retain its catalytic activity, with $88 \%$ yield of product $\mathrm{C} 1$ in the $5^{\text {th }}$ catalytic cycle, the physical mixture of the two monofunctional catalysts suffered from fast deactivation, with only a $26 \%$ yield of product $\mathrm{C} 1$ obtained in the third catalytic cycles. Presumably, the interactions between the Pd NPs and the surface of the silica support, without any organic groups, are relatively weak and cannot prevent aggregation and leaching of the Pd species during the catalysis. On the other hand, the presence of pendant aminopropyl moieties stabilized Pd nanoparticles within the mesoporous channels, allowing the hybrid to retain its performances over more catalytic cycles. TEM images recorded on the bifunctional hybrid after five catalytic cycles enforce this hypothesis (Fig 5.2 C): the nanoparticles appear to be well dispersed throughout the porous channels of the support and no macro-aggregates are visible. ${ }^{16}$

In another study, Johnston et al. immobilized Pd nanoparticles onto silica-based mesocellular foams (MCFs). MCF feature a three-dimensional structure with high surface area $\left(500-800 \mathrm{~m}^{2} / \mathrm{g}\right)$ and interconnected pores of size tunable between 25 and $42 \mathrm{~nm}$, interconnected by $9-22 \mathrm{~nm}$ windows. ${ }^{17}$ The authors used a support featuring $29 \mathrm{~nm}$ pores and $15 \mathrm{~nm}$ windows; moreover, the silica support was functionalized with aminopropyl groups, in order to promote the interactions between palladium nanoparticles and the support. TEM microscopy on the resulting material showed a narrow particle size distribution (1-2 nm, Fig. 5.3 A). The hybrid was found to be a highly efficient heterogeneous catalyst for the oxidation of alcohols. Primary and secondary benzylic alcohols were oxidized to the corresponding carbonyl compounds in high yields, using air as the source of molecular oxygen. The catalyst was found to be highly stable and was recycled several times without any leaching of the metal. ${ }^{18}$ 


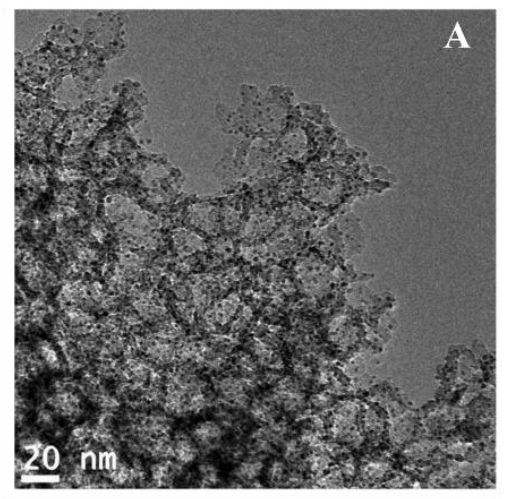

B

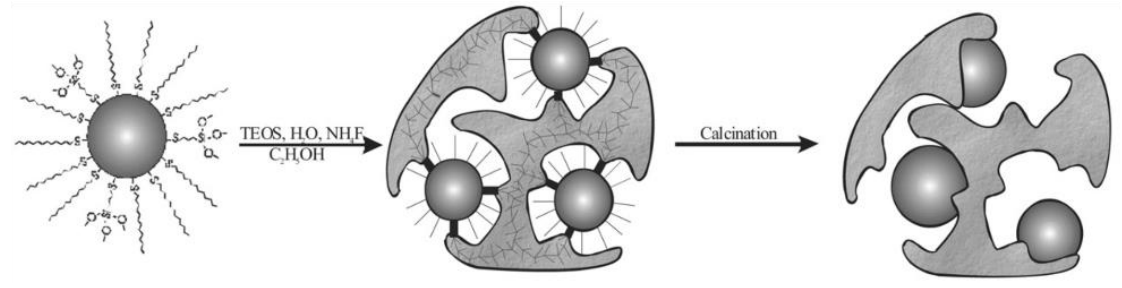

Figure 5.3 A: TEM images of MCF-supported Pd NPs. ${ }^{18}$ B: Schematic representation of the synthesis of silica-supported Pd NPs. ${ }^{19}$ C: Schematic representation of the synthesis of Pd/SBA-16. ${ }^{20}$

Silyl-derivatives can also be used to prepare and stabilize Pd NPs. Budroni et al. prepared a material composed of palladium nanoparticles embedded into amorphous silica. Firstly, they prepared Pd nanoparticles in an inverse microemulsion system, reducing $\mathrm{PdCl}_{2}$ with hydrazine and stabilizing the metal particles with a mixture of two thiols, 1dodecanethiol (DT) and 3-mercaptopropyltrimethoxysilane (MPMS). Subsequent cocondensation of TEOS in presence of the stabilized nanoparticles allowed to incorporate the Pd NPs into a silica matrix. The alkoxy silyl groups of the MPMS molecules promoted the intimate mixture of palladium nanoparticles and the silica precursor. Lastly, calcination in air and reduction in $\mathrm{H}_{2} / \mathrm{N}_{2}$ mixture led to the final material (Fig. $5.3 \mathrm{~B}$ ). ${ }^{19}$

A rather interesting approach to the incorporation of Pd nanoparticles was reported by Ma and co-workers. They used an amino-functionalized SBA-16 as support and reduced $\mathrm{Pd}(\mathrm{OAc})_{2}$ precursor by chemical reduction with $\mathrm{NaBH}_{4}$ (Fig. $5.3 \mathrm{C}$ ). $\mathrm{SBA}-16$ is an ordered mesoporous material with cage-like structures. ${ }^{21}$ This mesoporous silicas has tunable cage size $(4-10 \mathrm{~nm})$ and pore entrance size generally smaller than $4 \mathrm{~nm}$. Each nanocage is interconnected by eight neighboring pore entrances in three special dimensions. Besides hosting metal complexes, isolated nanocages of SBA-16 can limit the growth of metal nanoparticles by the spatial restrictions. ${ }^{20,22}$ 
Based on the literature on the topic, it appears that $-\mathrm{NH}_{2}$ groups play a crucial role in stabilizing small Pd nanoparticles. Their ability to coordinate palladium through the $\mathrm{N}$ lone electron pair and the steric blockage, provided by the aminopropyl pendant fragments as a whole, limits particle migration on the surface of the support, ultimately hindering particle aggregation and leaching. Moreover, the base character of the amine groups opens up to the use of these hybrids as multi-functional catalysts for cascade reactions. 


\subsection{Silica-supported Pd organometallic complexes}

Another way to incorporate Pd species onto solid supports is the preparation of silicasupported organometallic complexes based on Pd. As mentioned in Chapter 1, organometallic complexes have been used to catalyze an array of different reactions spacing from $\mathrm{C}-\mathrm{C}, \mathrm{C}-\mathrm{N}$ and $\mathrm{C}-\mathrm{O}$ bond formation to oxidations/reductions, to asymmetric reactions. The high activity and selectivity that can be achieved by these catalysts relies on the effect of the ligands which, by coordinating the metal atom through their free electrons, affect its atomic orbitals in a specific way. This influence can be beneficial for the catalytic activity of the metal complex in a specific reaction. Ligands can also affect the catalytic activity in other ways. They can interact with functional groups present on the substrate molecules (and not interested in the reaction pathway) through weak bonds, promoting the special vicinity between the active site and the functional group that is meant to react with; moreover, if properly designed, they can sterically promote asymmetric catalysis. ${ }^{3,6,23,24}$ Two classed of Pd organometallic complexes that are of particular interest for this Doctoral Thesis are NHC-based and BAM-BINAP-based complexes.

\subsubsection{Pd-based N-Heterocyclic Carbenes (NHCs)}

Carbenes are neutral compounds featuring a divalent carbon atom characterized by sixelectron valency, four of them being involved in $\sigma$-bonds, while the other two remaining located at the central carbon. ${ }^{25}$ Specifically, the two electrons are paired in the highest occupied molecular orbital (HOMO) $\sigma$, leaving a free $\mathrm{p}_{\pi}$ orbital. For this reason, these are called singlet carbenes. The kinetic stability of singlet carbenes can be enhanced by the introduction of bulky substituents in $\alpha$-position to the carbene center. Thermodynamic stabilization of the singlet state can also be achieved, by interaction of the carbon orbitals, $\sigma$ and $\mathrm{p}_{\pi}$, with specific substituents. For example, $\sigma$-electron-withdrawing and $\pi$-electron-withdrawing substituents stabilize the $\sigma$ orbital by mesomeric and inductive effects, respectively, whereas $\pi$-electron donating groups enhance the energy of the vacant $\mathrm{p}_{\pi}$ orbital (Fig. 5.4). Combination of these contributions produce an increase in the $\sigma-\mathrm{p}_{\pi}$ gap, in turn favoring the singlet ground state multiplicity. Triplet carbenes also exist but will not be discussed in this manuscript. ${ }^{26}$

A

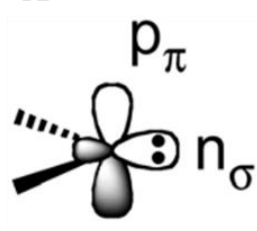

B

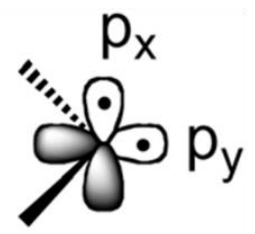

C

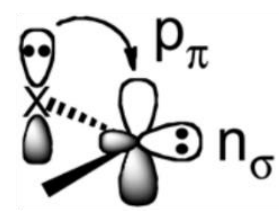

Figure 5.4: Singlet (A) and triplet (B) carbenes. Stabilization of the singlet ground state by interaction of the $p_{\pi}$ orbital with a $\pi$-electron donating $X$ group (C). Adapted from ref. 27. 
N-heterocyclic carbene (NHC) are the most widely studied class of carbenes, due to the stabilizing push-push mesomeric effect, in which the lone pairs of both $\mathrm{N}$ atoms interact with the $\mathrm{p}_{\pi}$ orbital of the carbon atom (Fig. 5.5). The stronger electron affinity of nitrogen, relative to carbon, cause an inductive effect that also contributes in maintaining the neutrality of the carbene center, increasing its stability. ${ }^{27}$

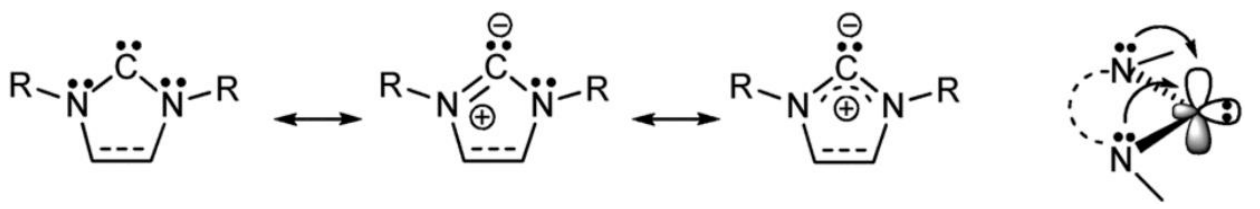

Figure 5.5: NHCs structure and mesomeric stabilization of the singlet ground state. Adapted from ref. 27.

Carbenes are reactive compounds and have been used for a number of catalytic reactions including benzoin condensation, ${ }^{28}$ transesterification, ${ }^{29}$ ring opening reactions, ${ }^{30}$ amongst all. Moreover, NHCs can act as ligand coordinating a metal through the lone electron pair. The success of NHCs as ligands in transition metal chemistry and catalysis is due to their ability to form particularly strong metal-carbon $\sigma$ bonds, which are usually stronger than the metal-phosphorus bonds of the corresponding phosphine complexes. ${ }^{31,32}$ Among the metal-NHC complexes developed, Pd-NHC complexes stand out due to their applications in cross-coupling reactions. The vast majority of these complexes are synthesized either by (i) substitution of a labile ligand with the free carbene or (ii) transmetallation from Ag complexes or (iii) in situ generation of the free carbene from an imidazolium salt in the presence of a palladium source.

Pd-NHC complexes have been obtained also as heterogeneous catalysts. For example MOF MIL-101(Cr) was functionalized with Pd-NHC moieties; ${ }^{33}$ in another study, a RuNHC pre-catalyst was modified and used as building block to obtain a coordination polymer. ${ }^{34}$ More of interest to this Doctoral Thesis, Pd-NHC complexes have also been supported onto silica materials. Varma et al. prepared a Pd-NHC-based organosilica, synthesizing firstly the silyl-derivative of the carbene, then promoting the coordination of the metal and then carrying out a sol-gel process catalyzed by $\mathrm{NH}_{4} \mathrm{~F}$ (Fig. 5.6). The integrity of the organic building block was confirmed by ${ }^{13} \mathrm{C}$ CP/MAS NMR. However, the catalyst exhibited a BET surface area of only $5 \mathrm{~m}^{2} / \mathrm{g}$. As mentioned in Chapter 1 , bridged silsesquioxane are prone to assemble in an ordered fashion whenever they can act as rigid building blocks, i.e. whenever they are constituted by unsaturated bonds that block the rotation of the atoms around the bond axes. It appears that in the case of the silyl-derivatives reported in this study, the flexible propyl linkers allowed conformational rearrangements of such building blocks, which favored the formation of non-porous solids. 

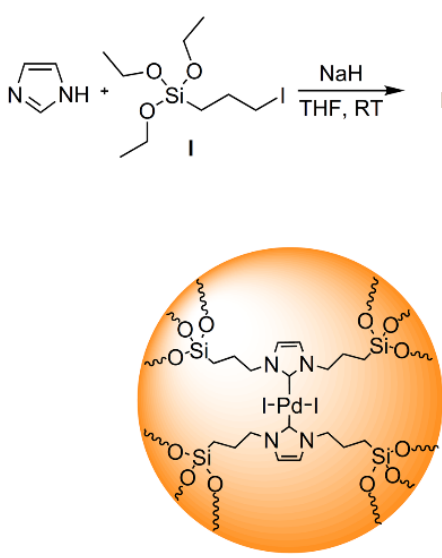

Pd-NHC Organic Silica (V)
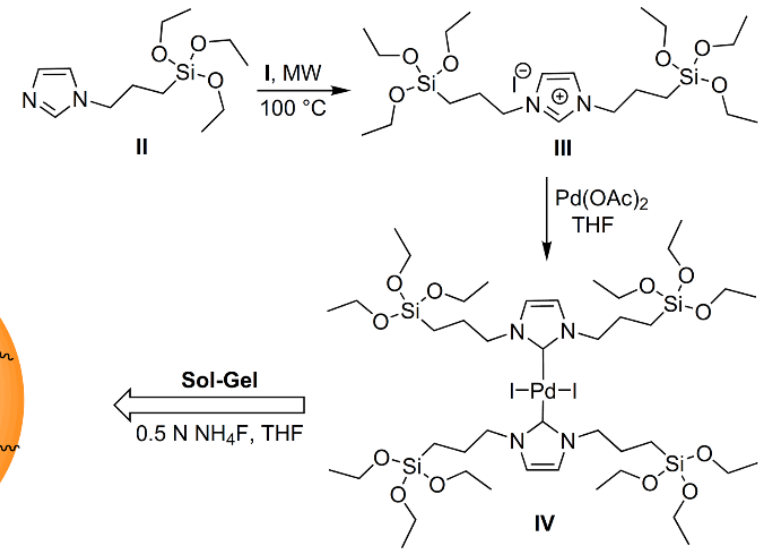

Figure 5.6: Preparation of Pd-NHC organosilica. ${ }^{35}$

The hybrid catalyst was tested in Heck and Suzuki coupling using different substrates, showing good catalytic activity. However, it should be pointed out that considering the non-porous nature of the solid, the majority of the active sites was most likely inaccessible by the substrate molecules. ${ }^{35}$

\subsubsection{Pd-BINAP complexes}

2,2'-bis(diphenylphosphino)-1,1'-binaphthyl (BINAP) is an organophosphorus compound that has been widely used as ligand in organometallic complexes. If only one enantiomer is used as ligand, the resulting complex can exhibit enantioselectivity (Fig. 5.7). The implementation of Rh-BINAP complexes in asymmetric hydrogenation reactions was worth Ryōji Noyori the 2001 Noble Prize in Chemistry. ${ }^{36}$

Since then, organometallic catalysts based on this ligand have been greatly used for a range of reactions. Palladium is surely one of the most used metals, in combination with BINAP ligands, to obtain highly active organocatalysts. Recent example of catalytic processes that benefitted from the presence of this type of catalyst include the 1,4-difunctionalization of naphthalene derivatives, ${ }^{37}$ the synthesis of enantiomers of $\mathrm{CF}_{3}$-substituted heterocycles by ring-expansion reaction, ${ }^{38}$ the synthesis of chiral hydantoin derivatives,${ }^{39}$ one-pot sequential Sonogashira and Cacchi reactions, ${ }^{40}$ double $\mathrm{N}$-arylation of primary amines to synthesize phenothiazines and phenoxazines, among others. ${ }^{41}$ 

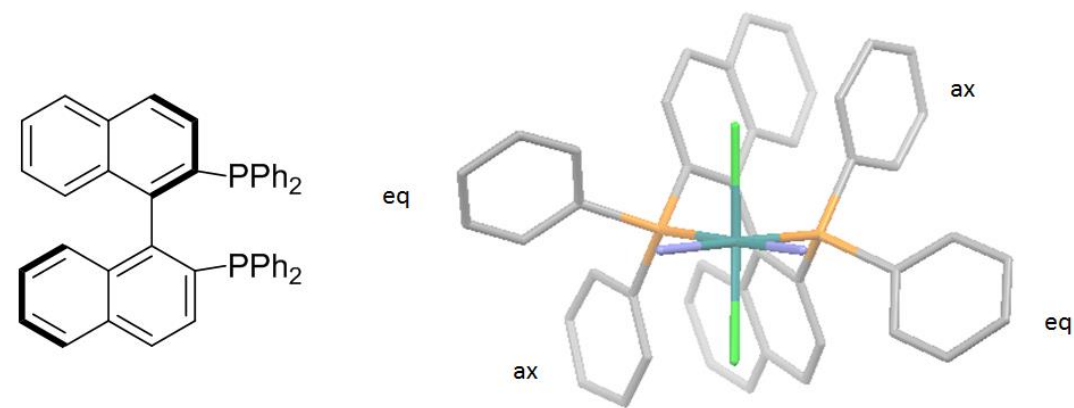

Figure 5.7: Structure of 2,2'-bis(diphenylphosphino)-1,1'-binaphthyl (BINAP).

Complexes featuring BINAP as ligand have also been bounded to solid support to achieve heterogeneous hybrid catalysts. Zhaojun et al. reported on the synthesis of BINAP-copper supported on hydrotalcite. The synthesis was carried out firstly by obtaining the dimer $\left[\mathrm{Cu}\right.$ (binap) $\Pi_{2}$ and then by wet impregnation onto the hydrotalcite support. The hybrid showed high efficiency in catalyzing dehydrogenative cyclization and borrowing hydrogen reactions under water or solvent-free conditions. Moreover, it showed recyclability up to five catalytic runs and negligible leaching of the metal, in spite of the fact that the $[\mathrm{Cu} \text { (binap) }]_{2}$ moieties were not bounded to the support through covalent bonds. ${ }^{42}$ In a subsequent study, the catalyst proved to have a wide catalytic scope, with good catalytic performances in the synthesis of 2-sulfanylphenols and arylthioquinones through a cascade $\mathrm{C}-\mathrm{H}$ hydroxylation and further oxidation process, starting from benzenethiol and phenylboronic acid derivatives. ${ }^{43}$ In another study, Reimann et al. used the enantioselective allylic alkylation of $(E)$-1,3-diphenylallyl acetate to study the heterogeneous/homogenous character of $(R)$-BINAP-palladium complex supported onto $\mathrm{Al}_{2} \mathrm{O}_{3}$. The catalyst was prepared in situ, mixing commercial $\mathrm{Pd} / \mathrm{Al}_{2} \mathrm{O}_{3}$ and $(R)$ BINAP in the reaction vessel. The study suggested that the material behaved as heterogeneous catalyst. Oxidation and consequent leaching of the Pd was prevented by the reaction conditions (THF and dioxane were used as solvents, and nucleophile sodium dimethyl malonate reduced Pd species that were oxidized during the reaction); the authors also suggested that BINAP itself could also consume surface oxygen. ${ }^{44}$ 


\subsection{Furfural as "green" building block}

In the last decades, the development of sustainable chemical processes and the use of green and bio-based chemicals have become more and more urgent. In this regard, furfural (FAL) obtained from biomass is one of the most promising building block that can be used to access a variety of relevant industrial products and intermediates (Fig. 5.8). Among these, the aldol condensation of FAL with ketones is implemented within the food industry and has been used for the synthesis of value-added liquid fuels.

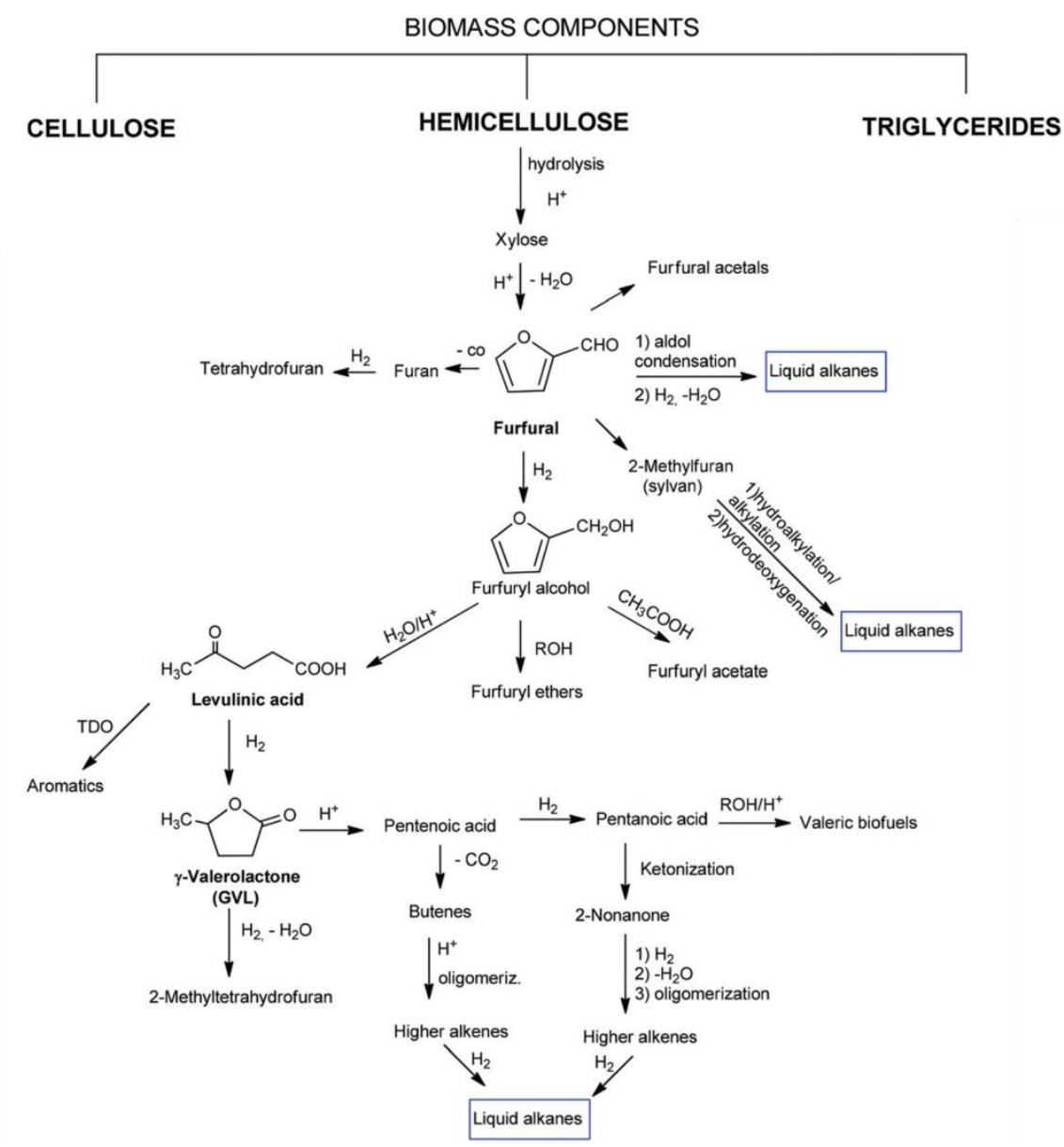

Figure 5.8: Processing options to convert biomass into valuable fuels and fuel additives. Adapted from ref. 45. 
Among the many catalytic processes that have been proposed starting from biomassderived platform molecules, routes involving the aldol condensation reaction of furfural with a ketone followed by a second reaction carried out in tandem fashion and in a single reactor are of particular industrial interest. For instance, jet fuels, ${ }^{46-49}$ and monomers for polyester and epoxy resins,${ }^{50}$ have been prepared starting from the aldol condensation reaction of furfural with either acetone, methyl isobutyl ketone (MIBK) or cyclopentanone, followed by either hydrogenation or hydrodeoxygenation under solvent-free conditions. Besides, one-pot reactions have been reported for the synthesis of furan- and THF-derived amines by one-pot aldol condensation reaction of furfural with ketones, followed by reductive amination with $\mathrm{NH}_{3}$ and $\mathrm{H}_{2}$ (Fig. 5.9). ${ }^{51,52}$

Nowadays, aldol condensation reactions in the chemical industry are mainly carried out under homogeneous catalysis conditions, using mineral bases such as $\mathrm{KOH}$ and $\mathrm{NaOH}$, which imply the generation of waste and the non-recyclability of the catalysts. In the pursuit of a greener alternative, many efforts have been put in designing heterogeneous catalysts such as metal oxides, ${ }^{53,54}$ zeolites ${ }^{55,56}$ and hybrid organic-inorganic catalysts. ${ }^{57,58}$ The employment of multi-functional hybrid catalysts containing base and redox sites represents a valid option for further development of sustainable biomass transformations, starting from furfural-derivative substrates.

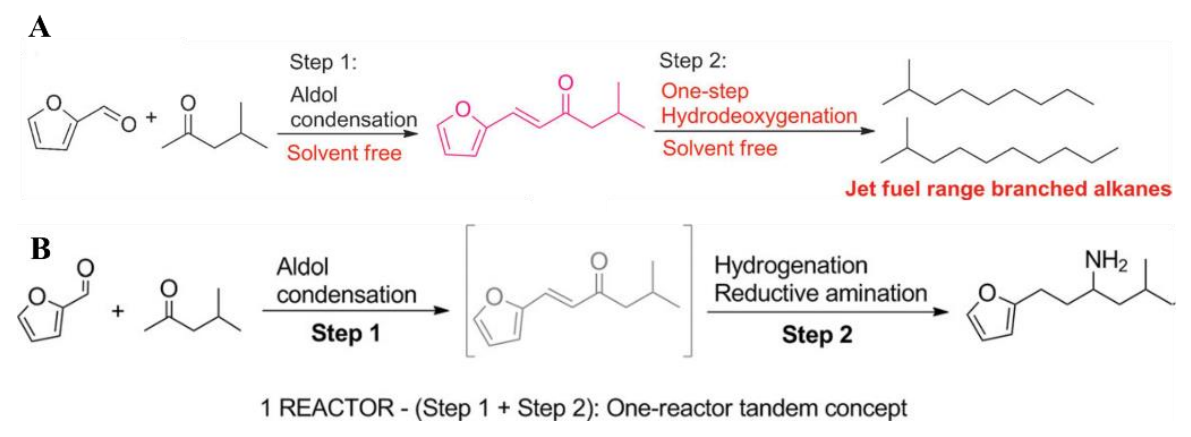

Figure 5.9 A: Schematic representation of the synthetic route to obtain jet-fuel-range branched alkanes from furfural. Adapted from ref. 49. B: Schematic representation of the synthesis of 1-(furan-2-yl)-4-methylpentan-2-amine from furfural. Adapted from ref. 51. 


\subsection{Scope of this work}

In this Chapter, the synthesis of multi-functional hybrid organic-inorganic catalysts will be described. The hybrids were designed upon the needs of and/or in collaboration with the industrial partners of the MULTI2HYCAT European project. This Chapter is meant to highlight the complexity of the design of multi-functional catalysts, briefly describing some of the possible approaches that can be implemented in their synthesis. Three multifunctional catalysts will be described.

- The first one will be a mesoporous silica functionalized with a base active (aminopropyl species) and palladium nanoparticles as second catalytic site. Pure silica MCM-41 will be chosen as support, due to its high surface area and reactive surface, onto which silyl-derivatives can be easily grafted.

- The catalyst will be characterized and its properties will be compared with those of the mono-functionalized catalyst baring aminoproyl residues only. Specifically, Xray powder diffraction, transmission electron microscopy, $\mathrm{N}_{2}$ physisorption analysis at $77 \mathrm{~K},{ }^{13} \mathrm{C} \mathrm{CP} / \mathrm{MAS}$ NMR spectroscopy, elemental analysis and ICP/OES will be performed to study structural, morphological, textural and chemical properties of the hybrids. Moreover, a distribution of the palladium nanoparticles size will be estimated from TEM images.

- The bi-functional hybrid will be tested as catalyst in a tandem reaction consisting of the aldol condensation between furfural and methyl isobutyl ketone (MIBK), followed by hydrogenation of the aldol intermediate. As highlighted above, this reaction is particularly interesting for biomass conversion and it is selected in agreement with other partners of the consortium. Several catalytic tests will be performed with the aim of exploring the effect of different variables such as temperature, catalyst amount, furfural/MIBK ratio and the presence of $\mathrm{H}_{2}$ from the beginning of the tandem catalytic process. Preliminary conclusions will be drawn in the prospect of a more thorough catalytic evaluation, to be carried out in flow regime using a fixedbed reactor.

- In the last Section of this Chapter, the synthesis of two bi-functional catalyst including two active sites will be reported. The first one will be prepared from a bis-Nheterocyclic carbene in which the precursor of the carbene (1-methylimidazole) will be previously functionalized with alkoxysilyl groups through a propyl linker. Such silyl-derivative will be incorporated onto commercial porous silica, together with another silyl derivative baring primary amine, through one-pot sequential grafting procedure. The second hybrid will be prepared starting from a custom-designed platform molecule constituted by a di-aminated 2,2'-bis(diphenylphosphino)-1,1'binaphthyl (BINAP) derivative, prepared by a partner of the consortium. These two hetereogeneous catalysts are part of an on-going research project. For that, in some cases details on the synthesis will be omitted. The catalysts will be tested in a tandem 
process involving the Suzuki coupling between benzeneboronic acid and 4-bromobenzaldehyde followed by the aldol condensation between the product and acetone. This Section is meant to provide insights on the different synthetic strategies that can be implemented in the design of multi-functional hybrid catalysts featuring relatively delicate active sites, such as transition metal complexes. Real examples drawn from the work carried out by the MULTI2HYCAT consortium will be used to this scope. 


\subsection{Experimental}

\subsubsection{Synthesis of the materials}

Synthesis of the MCM-41 support: $13.66 \mathrm{~g}$ of cetyltrimethylammonium bromide (7.6 $\mathrm{mmol}$ ) were dissolved in $91 \mathrm{~mL}$ of distilled water, together with $23.66 \mathrm{~g}$ of tetramethylammonium hydroxide solution $\left(25 \mathrm{wt} \%\right.$ in water) in a $250 \mathrm{ml}$ flask at $40{ }^{\circ} \mathrm{C}$. The mixture was vigorously stirred until complete dissolution of the surfactant. $15 \mathrm{~g}$ of Aerosil 200 were added and the resulting slurry was stirred for $1 \mathrm{~h}$, at room temperature. The gel was then transferred into a stainless steel autoclave and reacted for $24 \mathrm{~h}$ under static conditions, at $135{ }^{\circ} \mathrm{C}$. The solid obtained was filtered and washed abundantly with distilled water and finally dried at $100{ }^{\circ} \mathrm{C}$ overnight. The solid was then calcined, heating at $3{ }^{\circ} \mathrm{C} / \mathrm{min}$ under a flow of $\mathrm{N}_{2}$ until $540{ }^{\circ} \mathrm{C}$, temperature at which the gas flow was switched to air. The temperature was kept for $6 \mathrm{~h}$ before allowing to cool down.

Synthesis of $\mathrm{NH}_{2}-\mathrm{MCM}-41: 2 \mathrm{~g}$ of calcined MCM-41 were placed in a Schlenck and outgassed at $150{ }^{\circ} \mathrm{C}$ for $2 \mathrm{~h}$. Dry toluene was added and the mixture was stirred at $\mathrm{rT}$ for a few minutes. After that, $1.3 \mathrm{mmol}$ of aminopropyl triethoxysilane $(295 \mathrm{mg}$ ) were added and the resulting mixture was reacted at $80^{\circ} \mathrm{C}$ for $24 \mathrm{~h}$. After this time, the mixture was filtrated and washed with $\mathrm{CH}_{2} \mathrm{Cl}_{2}$ and ethanol. The materials were finally dried overnight at $100^{\circ} \mathrm{C}$. In coherence with the nomenclature used in Chapter 4, the catalyst was named 1.

Synthesis of Pd nanoparticles supported onto $\mathrm{NH}_{2}-\mathrm{MCM}-41$ : catalyst $1(1.5 \mathrm{~g})$ and triethylammoniun acetate $(0.47 \mathrm{mmol}, 142 \mathrm{mg})$ were placed in a Schlenck under $\mathrm{N}_{2}$. $\mathrm{Pd}(\mathrm{OAc})_{2}(0.4 \mathrm{~mol} \%, 0.15 \mathrm{mmol}, 33.7 \mathrm{mg})$ was dissolved in dry THF $(10 \mathrm{~mL})$. The solution was filtered with a Teflon syringe filter $(0.45 \mu \mathrm{L})$ and poured into the Schlenck. The resulting mixture was refluxed for $4 \mathrm{~h}$ and stirred at room temperature overnight. After this time, the mixture was filtered and the solid was washed with ether and dried for $1 \mathrm{~h}$ at room temperature. The catalyst was named 1-Pd.

Synthesis of silyl-derivative of 1-methylimidazolium iodide: $1.3 \mathrm{mmol}$ of 3-iodopropyl trimethoxysilane $(387 \mathrm{mg})$ and the equivalent moles of 1-methylimidazole $(110 \mathrm{mg})$ were mixed in dry $\mathrm{CH}_{3} \mathrm{CN}$ under $\mathrm{N}_{2}$. The resulting solution was refluxed overnight. The solvent was then removed under reduced pressure and the gel was washed with diethyl ether.

Synthesis of Pd-BisNHC-silyl derivative: the silyl-derivative of 1-methylimidazolium iodide and $\mathrm{Ag}_{2} \mathrm{O}$ together with dry $\mathrm{CH}_{2} \mathrm{Cl}_{2}$ were placed in a Schlenck wrapped with tin foil under $\mathrm{N}_{2}$. The solution was refluxed for $4 \mathrm{~h}$. After this time, $\mathrm{PdCl}_{2}\left(\mathrm{CH}_{3} \mathrm{CN}\right)_{2}$ was added and the mixtures was stirred at room temperature overnight. Finally, the solvent was removed under reduced pressure and the resulting solid was extracted with ether. The compound was named Pd-BisNHC.

Synthesis of Pd-BisNHC-functionalized silica: $2 \mathrm{~g}$ of commercial silica gel Ultrasil were placed in a Schlenck under $\mathrm{N}_{2}$ at $150{ }^{\circ} \mathrm{C}$ for $2 \mathrm{~h}$. Dry $\mathrm{CHCl}_{3}$ was added and the mixture 
was stirred at room temperature for $1 \mathrm{~h}$. After that, $2 \%$ mol of Pd-BisNHC were added and the resulting mixture was refluxed for $12 \mathrm{~h}$. After this time, aminopropyl triethoxysilane ( $2 \mathrm{~mol} \%$ ) was added and the resulting mixture was refluxed for $24 \mathrm{~h}$. Finally, the mixture was filtrated and washed with $\mathrm{CHCl}_{3}$ and acetone. The resulting solid was dried overnight at $100{ }^{\circ} \mathrm{C}$. The hybrid material was named Pd-BisNHC-NH $\mathrm{N}_{2}-\mathrm{SiO}_{2}$.

5,5'-dimethylamino-2,2'-bis(diphenylphosphino)-1,1'-binaphthyl (BAM-BINAP): The compound was synthesized by an industrial partner of the MULTI2HYCAT consortium, CAGE Chemicals.

\subsubsection{Characterization}

XRD measurements were carried out using a Philips X'PERT diffractometer equipped with a proportional detector and a secondary graphite monochromator. Data were collected stepwise in the $2^{\circ} \leq 2 \theta \leq 20^{\circ}$ angular region: $0.02^{\circ} 2 \theta$ steps, $20 \mathrm{~s} / \mathrm{step}$ accumulation time, beam-in was produced from $\mathrm{Cu} \mathrm{K}_{\alpha}(\lambda=1.54178 \AA)$. C, N, S, and H relative amounts were determined using a Carlo Erba 1106 elemental analyzer. The BET specific surface area was calculated from the nitrogen physisorption data in the relative pressure range from 0.04 to 0.2 . The total pore volume was obtained from the amount of $\mathrm{N}_{2}$ adsorbed at $\mathrm{P} / \mathrm{P}_{0}$ of about 0.99 . The pore diameter and the pore size distribution were calculated using the Barret-Joyner-Halenda (BJH) method on the adsorption branch of the $\mathrm{N}_{2}$ isotherms. Solid state MAS NMR spectra were recorded at room temperature in a Bruker AV-400 spectrometer. ${ }^{1} \mathrm{H}$ to ${ }^{13} \mathrm{C}$ cross-polarization (CP) spectra were acquired using a $90^{\circ}$ pulse for ${ }^{1} \mathrm{H}$ of $5 \mu \mathrm{s}$, a contact time of $5 \mathrm{~ms}$, and a recycle of $3 \mathrm{~ms} .{ }^{13} \mathrm{C}$ spectra were recorded with a $7 \mathrm{~mm}$ Bruker $B L-7$ probe and at a sample spinning rate of $5 \mathrm{kHz}$. ${ }^{13} \mathrm{C}$ was referred to adamantine. Chemical shifts were reported in ppm and were referenced to the appropriate residual solvent peak. Transmission electron microscopy (TEM) micrographs were acquired with a JEOL JEM2100 F electron microscope operating at $200 \mathrm{keV}$. The samples were treated through the dispersion of the powders onto carbon copper grids. Metal contents were determined using a Varian 715-ES ICP-Optical Emission spectrometer, after dissolution of the solids in $\mathrm{HNO}_{3} / \mathrm{HCl} / \mathrm{HF}$ aqueous solution. Mass spectrometry was performed using a Acquity XevoQToF spectrometer.

\subsubsection{Catalysis}

Tandem reaction (aldol condensation + hydrogenation) between furfural (FAL) and methyl isobutyl ketone (MIBK): The reactions were carried out in a stainless steel reactor connected to a gas supply system and equipped with a heater and a mechanical stirrer. In a typical reaction, $1 \mathrm{mmol}$ of distilled furfural, $2 \mathrm{mmol}$ of methyl isobutyl ketone, 0.5 $\mathrm{g}$ of toluene and $10 \mathrm{~mol} \%$ of catalyst were stirred at $80{ }^{\circ} \mathrm{C}$ for $24 \mathrm{~h}$ (aldol condensation step). Secondly, 5 bar of $\mathrm{H}_{2}$ were added and the reaction was continued for $8 \mathrm{~h}$. The reaction was monitored by NMR analysis using $\mathrm{d} 3-\mathrm{CD}_{3} \mathrm{CN}$ as deuterated solvent. 


\subsection{Results and discussion}

\subsubsection{Bi-functional catalysts featuring Pd nanoparticles}

\subsubsection{Pd@NH $-M C M-41$}

A bi-functional catalyst featuring aminopropyl species together with palladium nanoparticles was developed. The catalyst was designed for a specific application, i.e. the aldol condensation between furfural (FAL) and methyl isobutyl ketone (MIBK) followed by hydrogenation of the aldol-intermediate, through tandem catalytic process.

The preparation of the catalyst was carried out following the scheme reported in Fig. 5.10. Firstly, aminopropyl triethoxysilane (APTS) was grafted onto pure silica MCM41 , following the procedure also implemented for synthesizing the catalysts described in Chapter 4 (catalyst 1). Then, palladium nanoparticles were prepared in situ and supported onto the catalyst. Specifically, $\mathrm{NH}_{2}-\mathrm{MCM}-41$ and triethylammonium acetate were mixed in a Schlenck, under $\mathrm{N}_{2}$ atmosphere. In parallel, palladium acetate was dissolved in dry THF. This solution was filtered with a Teflon syringe filter $(0.45 \mu \mathrm{L})$, in order to remove eventual agglomerates, and then added to the Schlenck. The mixture was refluxed for 4 hours, cooled down to room temperature and stirred overnight. Lastly, the suspension was filtered and the recovered solid was washed with diethyl ether and dried for $1 \mathrm{~h}$ at room temperature (catalyst 1-Pd).

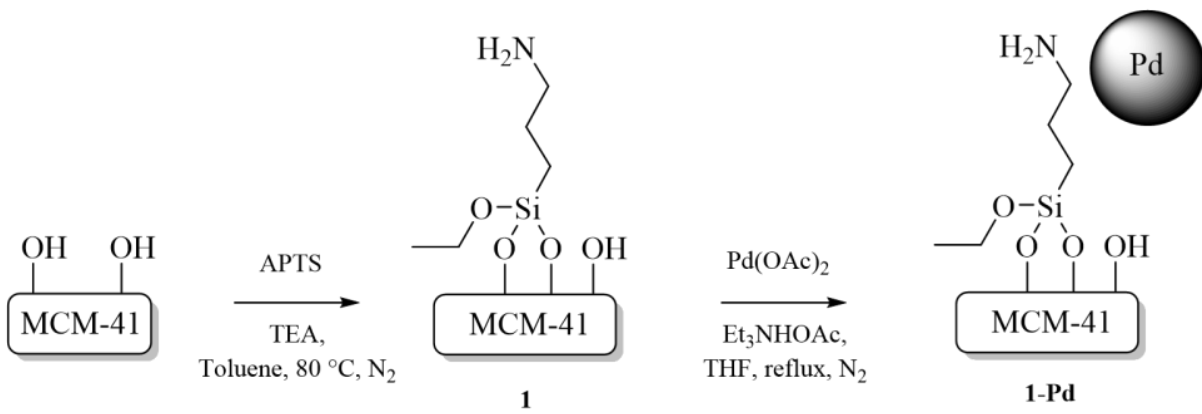

Figure 5.10: Schematic representation of the synthesis of $\mathrm{Pd} @ \mathrm{NH}_{2}-\mathrm{MCM}-41$.

Hybrid solids 1 and 1-Pd were characterized with a multi-techniques approach. Firstly, the structural integrity was accessed by X-ray powder diffraction (Fig. 5.11). Signals related to the presence of the M41S long-range hexagonal ordered structure are present, confirming that the incorporation of palladium does not affect the integrity of the mesoporous architecture. Focusing on the most intense signal, related to the (100) planes, no major changes in the peak position is observed upon Pd deposition. 


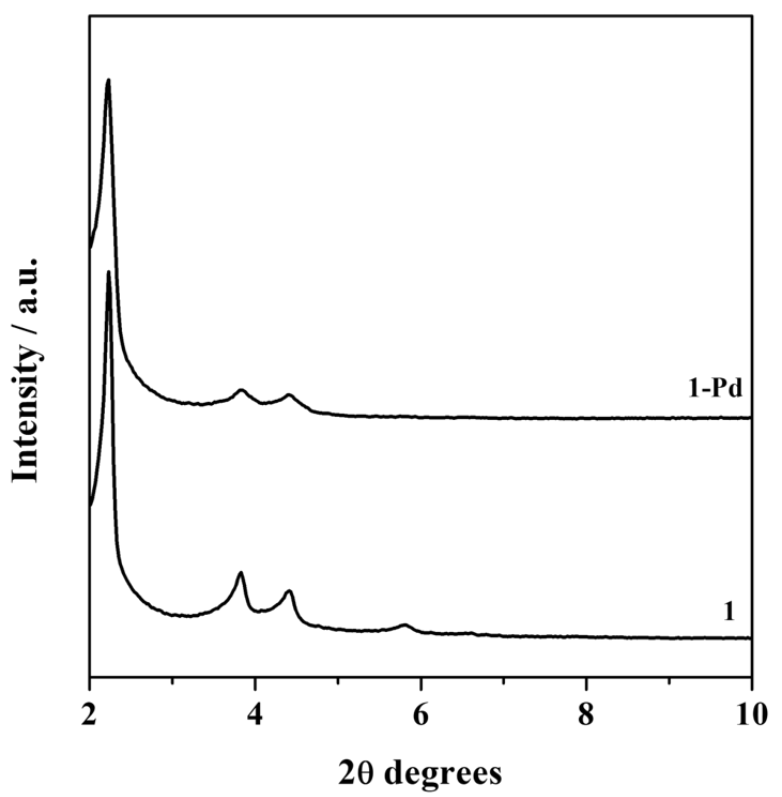

Figure 5.11: X-ray powder diffraction patterns of catalysts 1 and 1-Pd.

This might indicate a preferential location of the Pd nanoparticles on the external surface of the silica particles, as the partial occlusion of the mesoporous channels would cause a reduction in the diameter, substantially modifying diffraction pattern. However, the physical reason why non-crystalline long-range ordered porous structures can coherently diffract X-ray photons is the difference in matter density between the framework and the hollow pores; therefore, if only a small fraction of the pores was filled (which can be expected, considering the low target amount of $\mathrm{Pd}$ ), the vast majority of the photons of the beam-in would still be diffracted in a way that is analogous to that of the hybrid before Pd deposition.

Transmission electron micrographs were recorded on catalyst 1-Pd to observe the presence of Pd nanoparticles. Both bright-field (Fig. 5.12 A and B) and dark-field images (Fig. 5.12 C) clearly show a homogeneous distribution of nanoparticles throughout the portion of the sample analyzed. Importantly, no macro-aggregates are seen. Other images, not reported here, show analogous results. The size of 148 nanoparticles was measured over a total of 6 images and a particle size distribution was calculated (Fig. 5.12 D). The bar-chart shows a narrow distribution centered at 2-2.5 nm of diameter, with a maximum value of $4.5 \mathrm{~nm}$ measured. Interestingly, the mean value observed corresponds to the diameter of the mesoporous channels of the MCM-41 support. 

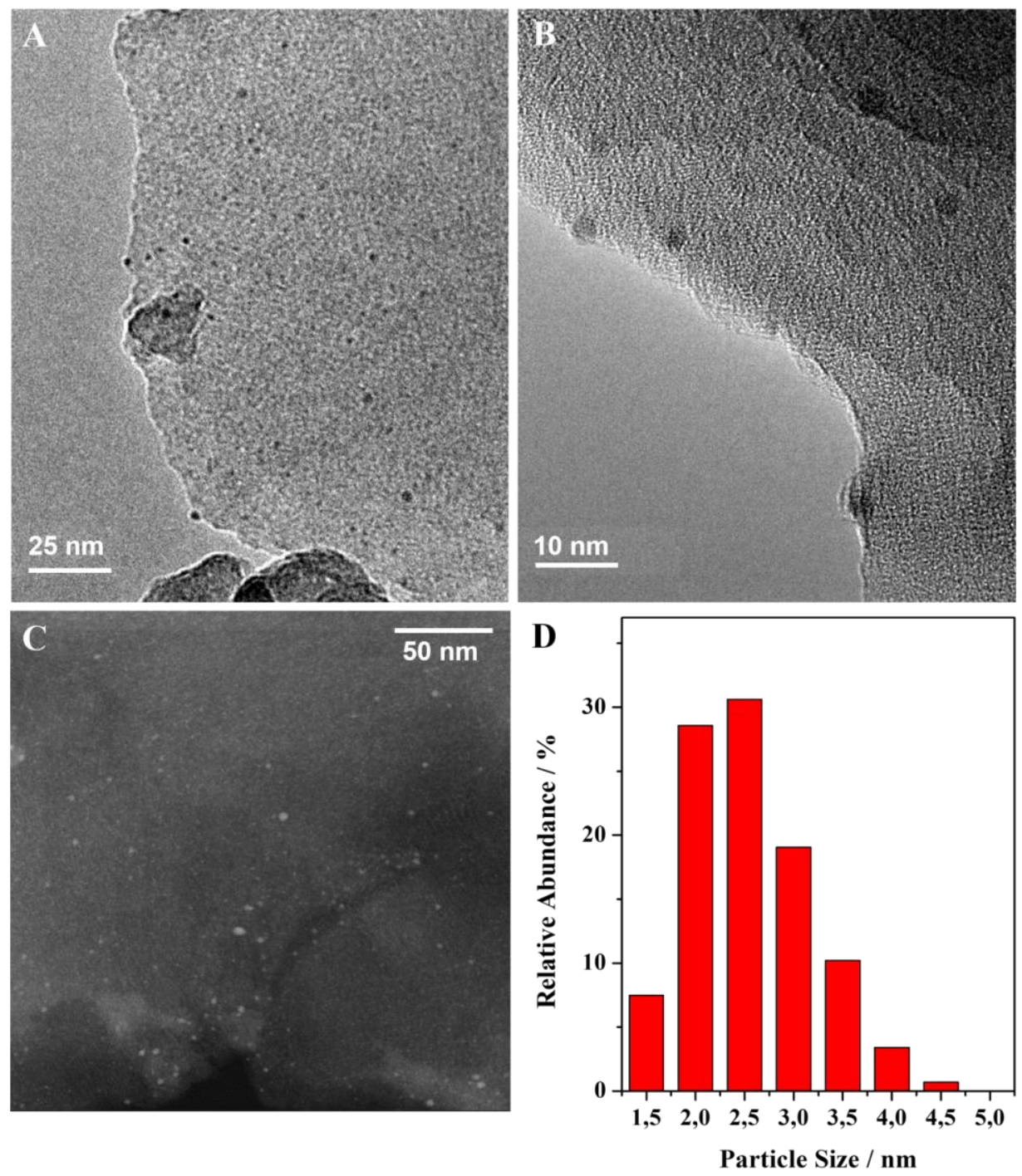

Figure 5.12: Transmission electron micrographs of catalyst 1-Pd recorded in bright-field (AB) and dark-field (C) modes. Particle size distribution calculated upon measuring the size of 147 particles over a total of 8 micrographs (D). 
The most likely interpretation of this finding is that nanoparticles forming within the channels can grow until reaching the size of the pore; the presence of the surface aminopropyl- pendant fragments stabilizes the particles in their position, hindering their migration throughout the channels by chemical and steric interactions.

$\mathrm{N}_{2}$ physisorption analysis at $77 \mathrm{~K}$ was recorded on the two hybrids, prior to and after Pd deposition, and compared with a pure silica MCM-41 support (Fig. 5.13). The three isotherms show a type-IV behavior, typical of mesoporous materials. Interestingly, the incorporation of aminopropyl- moieties and palladium NPs causes a shift of the inflection point (marked with a dotted line in the figure), in the region between 0,2 and $0,4 \mathrm{P} / \mathrm{P}_{0}$, which indicates the presence of smaller mesopores. Indeed, the pore size distribution, calculated using the BJH method on the desorption branch of the isotherm, shows the mean value of the pore size slightly down-shifting for 1 and 1-Pd catalysts. The pore volume values calculated with the $\mathrm{BJH}$ method also show a progressive occlusion of the channels (Table. 5.1). Coherently, the two-steps functionalization also causes a decrease in the specific surface area (calculated with the BET method and also reported in Table 5.1) from 1168 to 973 to $913 \mathrm{~m}^{2} / \mathrm{g}$.

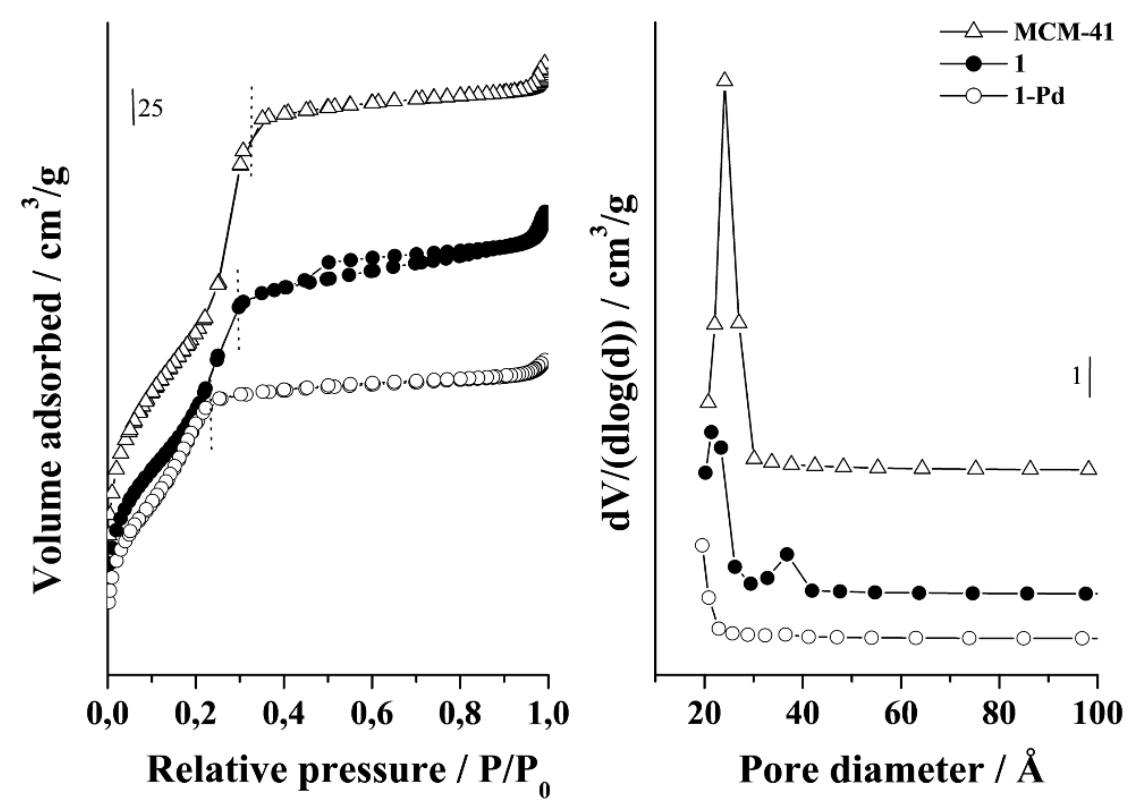

Figure 5.13: $\mathrm{N}_{2}$ physisorption isotherms at $77 \mathrm{~K}(\mathrm{~A})$ and $\mathrm{BJH}$ pore size distribution (B) of pure silica MCM-41, catalyst 1 and 1-Pd. 
Table 5.1: compositional and textural properties of the hybrids.

\begin{tabular}{lcccccc}
\hline Catalyst & ${ }^{\mathrm{a}} \mathrm{N} \%$ & ${ }^{\mathrm{a}} \mathrm{C} \%$ & ${ }^{\mathrm{b} P d} \%$ & $\begin{array}{c}\mathrm{SSA}_{\mathrm{BET}} \\
\left(\mathrm{m}^{2} / \mathrm{g}\right)\end{array}$ & $\begin{array}{c}\text { Mean pore } \\
\text { size } \\
(\AA)\end{array}$ & $\begin{array}{c}\text { Pore volume } \\
\left(\mathrm{cm}^{3} / \mathrm{g}\right)\end{array}$ \\
\hline 1-Pd & 1.3 & 8.8 & 0.8 & 913 & 20 & 0.13 \\
$\mathbf{1}$ & 1.1 & 3.3 & $/$ & 973 & 22 & 0.46 \\
MCM-41 & $/$ & $/$ & $/$ & 1168 & 24 & 0.74 \\
\hline
\end{tabular}

aelemental analysis; ${ }^{\mathrm{b}} \mathrm{ICP}-\mathrm{OES}$. Mean pore size and pore volume were calculated from the desoption branch.

ICP-OES analysis indicates a $0.8 \%$ wt. of palladium incorporated in the samples. Elemental analysis was also performed on the catalysts. An increase in the organic content is observed from 1 to 1-Pd catalysts, most likely related to triethylammonium acetate incorporated on the Pd nanoparticles. The integrity of the aminopropyl residues after the functionalization with Pd NPs was accessed by ${ }^{13} \mathrm{C}$ solid state CP/MAS NMR (Fig. 5.14).

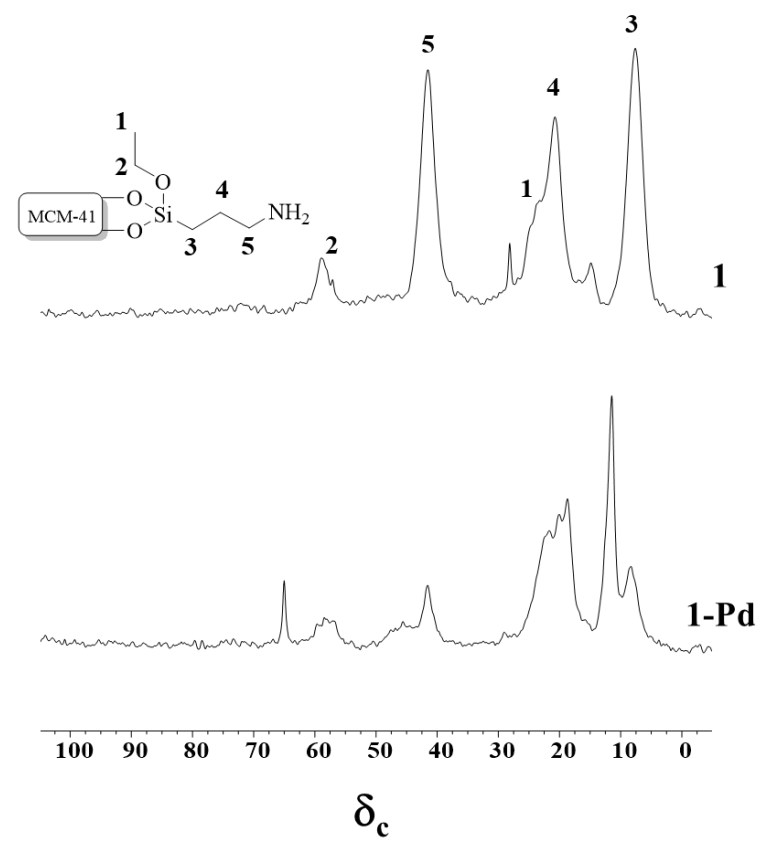

Figure 5.14: ${ }^{13} \mathrm{C}$ CP/MAS NMR spectra of hybrids 1 and 1-Pd. 
Signals related to the presence of aminopropyl moieties are observed in the spectra of catalysts 1-Pd, which confirms that the palladium nanoparticles deposition allows preserving the organic species. Additional signals are observed and ascribed to the presence of triethylammonium acetate, in line with the higher organic content observed by elemental analysis.

\subsubsection{Catalytic activity in tandem reaction}

Hybrid 1-Pd catalyst was tested in a tandem reaction consisting of the aldol condensation between furfural (FAL) and methyl isobutyl ketone (MIBK), followed by hydrogenation of the aldol intermediate (F-MIBK), resulting in three hydrogenated products: the desired 1-(furan-2-yl)-5-methylhexan-3-one (F-MIBK-ON), and the by-products 1-(furan-2-yl)5-methylhex-1-en-3-ol (F-MIBK-OL) and 1-(furan-2-yl)-5-methylhexan-3-ol (FMIBK-AN) (Fig. 5.15).

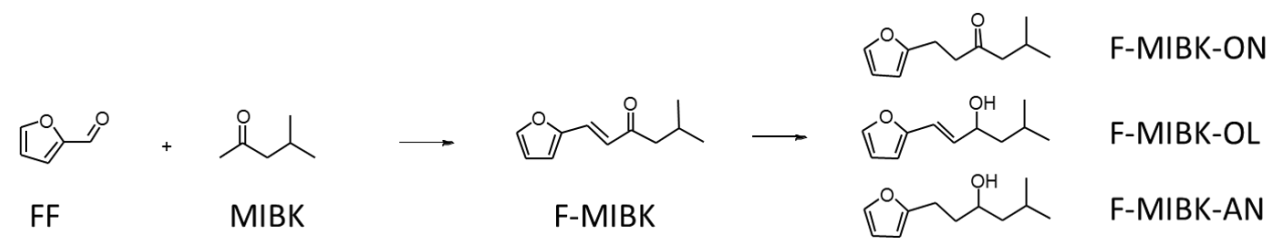

Figure 5.15: Schematic representation of the tandem reaction between furfural and methyl isobutyl ketone.

Several catalytic runs were performed in the attempt to elucidate the influence of different variables on conversion and selectivity. Results are reported in Table 5.2. Firstly, the reaction was carried out under "baseline conditions" (entry 1) and with a temperature of $80{ }^{\circ} \mathrm{C}$ for both steps. A $75 \%$ furfural conversion was observed, as well as a high selectivity toward F-MIBK-AN and a moderate yield of the desired F-MIBK-ON. By halving the amount of catalyst (entry 2) both the furfural conversion and selectivity decreased. Upon changing the FAL/MIBK ratio from $1 / 2$ to $1 / 4$ (entry 3 vs entry 2), the conversion of furfural increased; importantly, the selectivity seemed to be affected as well, with a similar yield of F-MIBK-AN and F-MIBK-OL at the end of the reaction. When the reaction was carried out in only one-step, adding $\mathrm{H}_{2}$ from the beginning, a high selectivity toward the most reduced product, i.e. F-MIBK-AN, was observed (entry 4).

After this initial screening, the temperature of both steps was raised from 80 to $100{ }^{\circ} \mathrm{C}$ (entry 5). No major changes were observed in the FAL conversion ( $80 \%$, vs $75 \%$ of entry 1). However, a drastic change in the selectivity was detected, with a $63 \%$ yield of the desired F-MIBK-ON product. Halving the $\mathrm{H}_{2}$ pressure introduced in the second step of the tandem reaction affected both conversion and selectivity in a negative fashion (entry 6). A catalytic run was performed in absence of solvent and with a FAL/MIBK ratio of 
1/6 (entry 7). An almost full conversion of furfural was observed; however, the reaction resulted similarly selective toward F-MIBK-ON and F-MIBK-AN. Lastly, a catalytic run at $100{ }^{\circ} \mathrm{C}$ and with the addition of 5 bar of $\mathrm{H}_{2}$ since the beginning of the reaction was performed, giving a furfural conversion of $94 \%$ and high selectivity toward the reduced F-MIBK-AN.

These preliminary results pave the way for a more thorough optimization of the catalytic condition that will be carried out in flow conditions using a fixed-bed reaction, in collaboration with the industrial partners of the MULTI2HYCAT European project.

Table 5.2: Catalytic activity of 1-Pd in the tandem reaction between FF and MIBK. ${ }^{a}$

\begin{tabular}{|c|c|c|c|c|c|c|}
\hline \multirow[b]{2}{*}{ Entry } & \multicolumn{2}{|c|}{ Reaction conditions } & \multirow{2}{*}{$\begin{array}{c}\text { FAL } \\
\text { conversion } \\
\%\end{array}$} & \multicolumn{3}{|c|}{ Yield $(\%)$} \\
\hline & Step 1 & Step & & $\begin{array}{c}\text { F-MIBK- } \\
\text { ON }\end{array}$ & $\begin{array}{c}\text { F-MIBK- } \\
\text { OL }\end{array}$ & $\begin{array}{c}\text { F-MIBK- } \\
\text { AN }\end{array}$ \\
\hline 1 & $80^{\circ} \mathrm{C} ; 24 \mathrm{~h}$ & $80^{\circ} \mathrm{C} ; 8 \mathrm{~h}$ & 75 & 21 & 10 & 44 \\
\hline $2^{\mathrm{c}}$ & $80^{\circ} \mathrm{C} ; 24 \mathrm{~h}$ & $80^{\circ} \mathrm{C} ; 24 \mathrm{~h}$ & 48 & 12 & 12 & 23 \\
\hline $3^{\mathrm{b}, \mathrm{c}}$ & $80^{\circ} \mathrm{C} ; 24 \mathrm{~h}$ & $80^{\circ} \mathrm{C} ; 24 \mathrm{~h}$ & 65 & 6 & 29 & 30 \\
\hline $4^{\mathrm{d}}$ & $80^{\circ} \mathrm{C} ; 24 \mathrm{~h}$ & $80^{\circ} \mathrm{C} ; 24 \mathrm{~h}$ & 57 & 1 & 18 & 38 \\
\hline 5 & $100^{\circ} \mathrm{C} ; 24 \mathrm{~h}$ & $100^{\circ} \mathrm{C} ; 8 \mathrm{~h}$ & 80 & 63 & 7 & 10 \\
\hline $6^{\mathrm{e}}$ & $100^{\circ} \mathrm{C} ; 24 \mathrm{~h}$ & $100^{\circ} \mathrm{C} ; 8 \mathrm{~h}$ & 61 & 33 & 10 & 18 \\
\hline $7^{\mathrm{f}}$ & $100^{\circ} \mathrm{C} ; 24 \mathrm{~h}$ & $100^{\circ} \mathrm{C} ; 8 \mathrm{~h}$ & 96 & 44 & 9 & 47 \\
\hline $8^{\mathrm{d}}$ & $100^{\circ} \mathrm{C} ; 24 \mathrm{~h}$ & $100^{\circ} \mathrm{C} ; 24 \mathrm{~h}$ & 94 & 3 & 15 & 82 \\
\hline
\end{tabular}

aReaction conditions: 1 mol FAL, FAL/MIBK (mol:mol)=1/2 mol, $0.5 \mathrm{~g}$ toluene, 5 bar $\mathrm{H}_{2}, 10$ mol\% of catalyst (amine); ${ }^{\mathrm{b}} \mathrm{FAL} / \mathrm{MIBK}(\mathrm{mol}: \mathrm{mol})=1 / 4 ;{ }^{\mathrm{c}} 5 \mathrm{~mol} \%$ catalyst $4 ;{ }^{\mathrm{d}} 5$ bar $\mathrm{H}_{2}$ added since the beginning of the reaction, ${ }^{\mathrm{e}} 2.5 \mathrm{bar} \mathrm{H}_{2},{ }^{\mathrm{f}} \mathrm{FAL} / \mathrm{MIBK}$ (mol:mol)= 1:6 and no solvent. 


\subsubsection{Bi-functional catalysts featuring palladium complexes}

\subsubsection{Pd-BisNHC@ $\mathrm{NH}_{2}-\mathrm{SiO}_{2}$}

A bi-functional catalyst featuring propylamino pendant fragments and Pd-BisNHC complex molecules is here described. The material was obtained following the reaction pathway depicted in Fig. 5.16, which involved the sequential grafting of two silyl-derivatives, each baring a catalytically active site.

Firstly, a silyl derivative of 1-methylimidazol was prepared through reaction with 3-iodopropyl trimethoxysilane (See Chapter 4 for details on the synthesis). Then, a palladium complex coordinated by two silyl-functionalized $\mathrm{N}$-heterocyclic carbene ligands was prepared (Pd-BisNHC).

$\mathbf{A}$

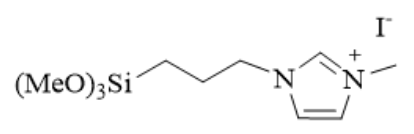

1) $\mathrm{Ag}_{2} \mathrm{O}, \mathrm{CH}_{2} \mathrm{Cl}_{2}, 40^{\circ} \mathrm{C}$

2) $\mathrm{PdCl}_{2}\left(\mathrm{CH}_{3} \mathrm{CN}\right)_{2}, \mathrm{RT}$

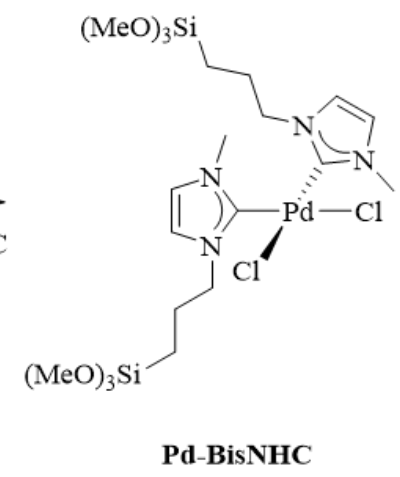

B
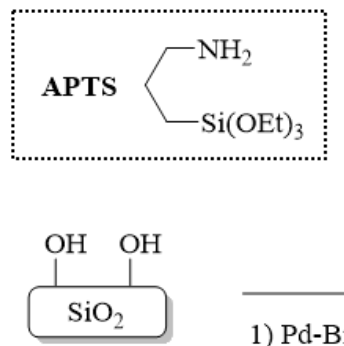

1) $\mathrm{Pd}-\mathrm{BisNHC}, \mathrm{CHCl}_{3}$, $12 \mathrm{~h}, 70^{\circ} \mathrm{C}$

2) APTS, $24 \mathrm{~h}, 70^{\circ} \mathrm{C}$

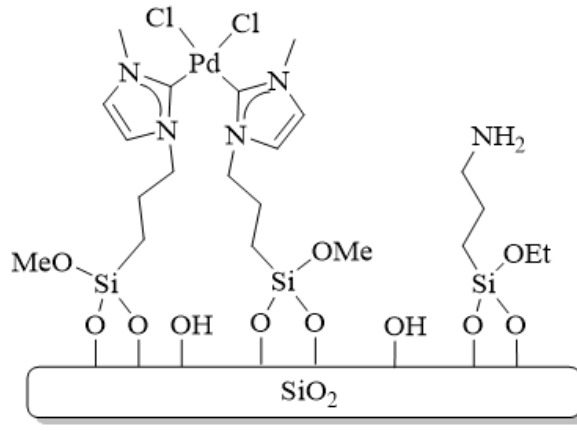

Pd-BisNHC@NH $2-\mathrm{SiO}_{2}$

Figure 5.16: Reaction pathways for the synthesis of silyl-derivative Pd-BisNHC (A) and of hybrid catalyst Pd-BisNHC@ $\mathrm{NH}_{2}-\mathrm{SiO}_{2}(\mathrm{~B})$. 
This was achieved by transmetallation from the Ag-NHC complex. The transmetalation of $\mathrm{Ag}-\mathrm{NHC}$ complexes is a widely used approach in the synthesis of metal-NHC complexes. Ag-NHC complexes can easily be prepared in good yields by the reaction of imidazolium salts and basic silver compounds (e.g., $\mathrm{Ag}_{2} \mathrm{O}, \mathrm{AgOAc}$ ) under mild conditions. Additionally, the convenient handling of Ag-NHC complexes provides an easy way to overcome difficulties associated with other methods, such as the handling of strong bases, harsh reaction conditions and complicated work-ups. ${ }^{59}$ Ultimately, hybrid catalyst Pd-BisNHC@ $\mathrm{NH}_{2}-\mathrm{SiO}_{2}$ was prepared through one-pot sequential grafting of Pd-BisNHC (12 h) and aminopropyl triethoxysilane (APTS, $24 \mathrm{~h}$ ).

Pd-BisNHC complex was characterized by electrospray ionization time-of-fly mass spectrometry (ESI-TOF MS). The $\mathrm{m} / \mathrm{z}$ signals, compared with those of the theoretical spectrum, confirm the presence of the compound (Fig. 5.17).
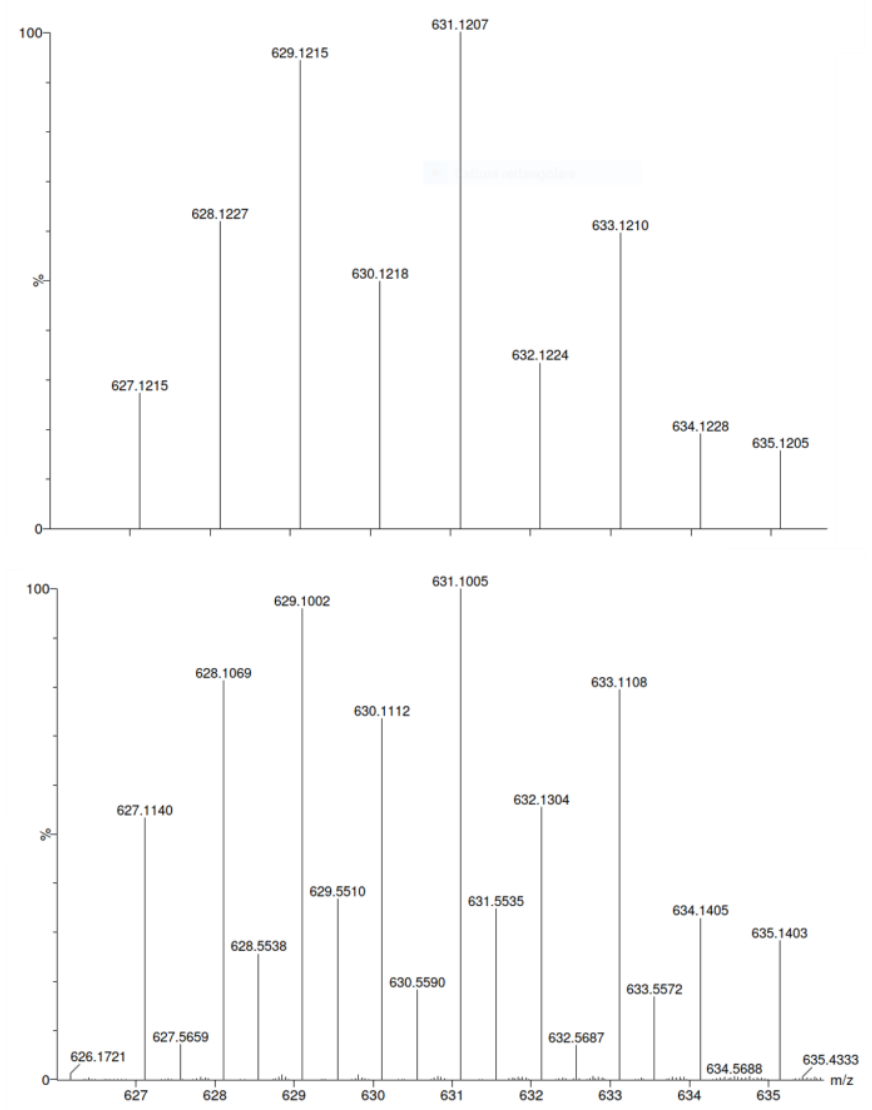

Figure 5.17: ESI-TOF MS spectrum of Pd-BisNHC compound. 
Hybrid catalyst Pd-BisNHC@ $\mathrm{NH}_{2}-\mathrm{SiO}_{2}$ showed a palladium content, measured by ICPOES, of $1,5 \%$ wt. ${ }^{13} \mathrm{C} \mathrm{CP} / \mathrm{MAS}$ NMR spectrum was recorded on the material (Fig. 5.18). The spectrum shows signal that are coherent with the desired structure. In the range between 0 and $60 \mathrm{ppm}$ signals related to the aliphatic carbon atoms of the propyl linkers are visible, as well as the signal of the $\mathrm{N}-\mathrm{CH}_{3}$ present on the imidazolium-derived ring. At higher chemical shifts, a composite signal centered at about $120 \mathrm{ppm}$ is visible and ascribed to the olephinic carbon atoms of the imidazolium-derived ring and to the carbene carbon atom, bounded to the palladium.
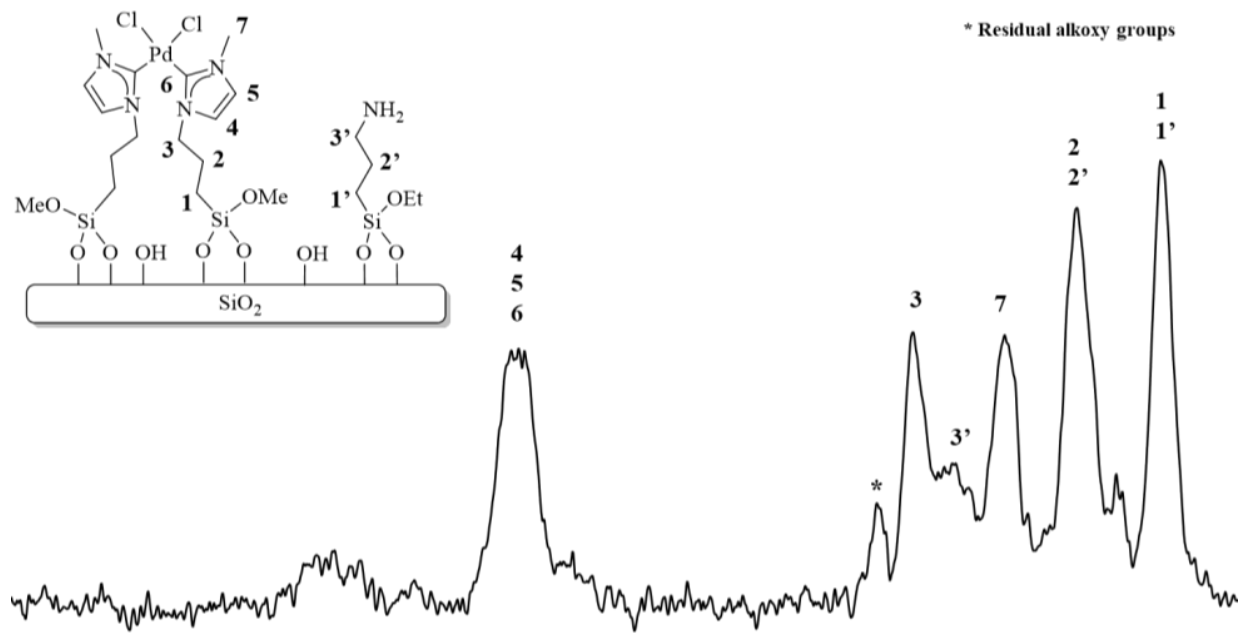

$\begin{array}{lllllllllllll}200 & 180 & 160 & 140 & 120 & 100 & 80 & 60 & 40 & 20 & 0 \\ & & & & & & & & & & & & \end{array}$

Figure 5.18: ${ }^{13} \mathrm{C}$ CP MAS NMR spectrum of hybrid Pd-BisNHC@ $\mathrm{NH}_{2}-\mathrm{SiO}_{2}$. 


\subsubsection{Pd-BAM-BINAP-MCM-41}

The synthesis of a bi-functional catalyst featuring primary amines and a palladium complex is here reported. This catalyst represents a different concept for the synthesis of multi-functional hybrid catalysts, compared to those described above. In the formers, the two functionalities came from two separate chemical building blocks, which were incorporated into a solid framework in one or two separate synthetic steps. In contrast, the hybrid described below was prepared starting for one platform molecule, featuring two different catalytic sites, i.e. base and metal. The catalyst was prepared from the precursor 5,5'-dimethylamino-2,2'-bis(diphenylphosphino)-1,1'-binaphthyl (BAM-BINAP, Fig. 5.19), provided by CAGE chemicals, an industrial partner of the MULTI2HYCAT consortium.

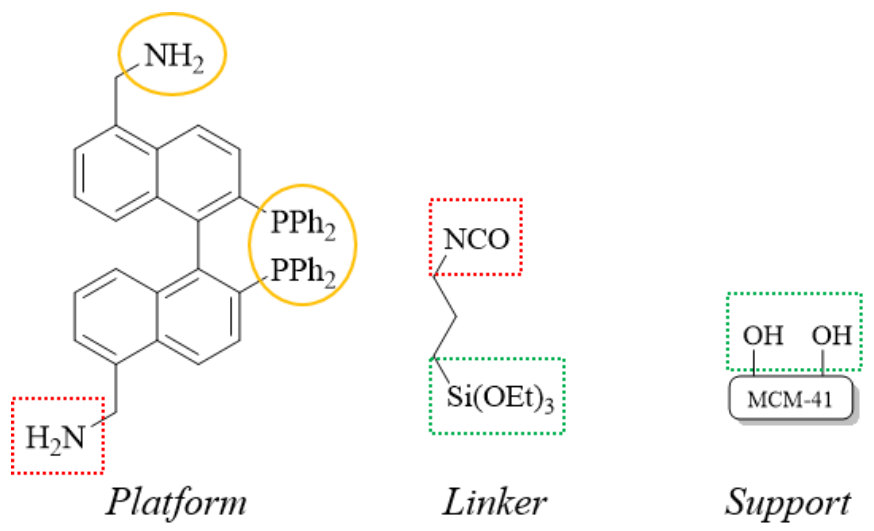

Figure 5.19: 5,5'-dimethylamino-2,2'-bis(diphenylphosphino)-1,1'-binaphthyl (BAMBINAP), provided by CAGE chemicals (platform). 3-isocyanatopropyltriethoxysilane (linker). MCM-41 (support).

The platform molecule featured two phosphine groups, meant to coordinate a metal atom, and two primary amine groups. Of those, one was meant to react and get bounded to the surface of a solid support through a linker (3-isocyanatopropyltriethoxysilane, Fig. 5.19), while the other was meant to be preserved and act as a base catalyst in a given catalytic process. However, the identical chemical nature of the two amine groups made impossible to carry out the selective reaction of only one of them with the isocyanate group of the linker, in homogeneous phase. To avoid this problem, an alternative strategy was implemented (Fig. 5.20). Firstly, the linker was firstly grafted onto MCM-41. In parallel, Pd-BAM-BINAP complex was synthesized. Lastly, a tethering reaction between the anchored isocyanatopropyl- groups and one of the amine groups of the complex was performed to achieve Pd-BAM-BINAP-MCM-41 material. 

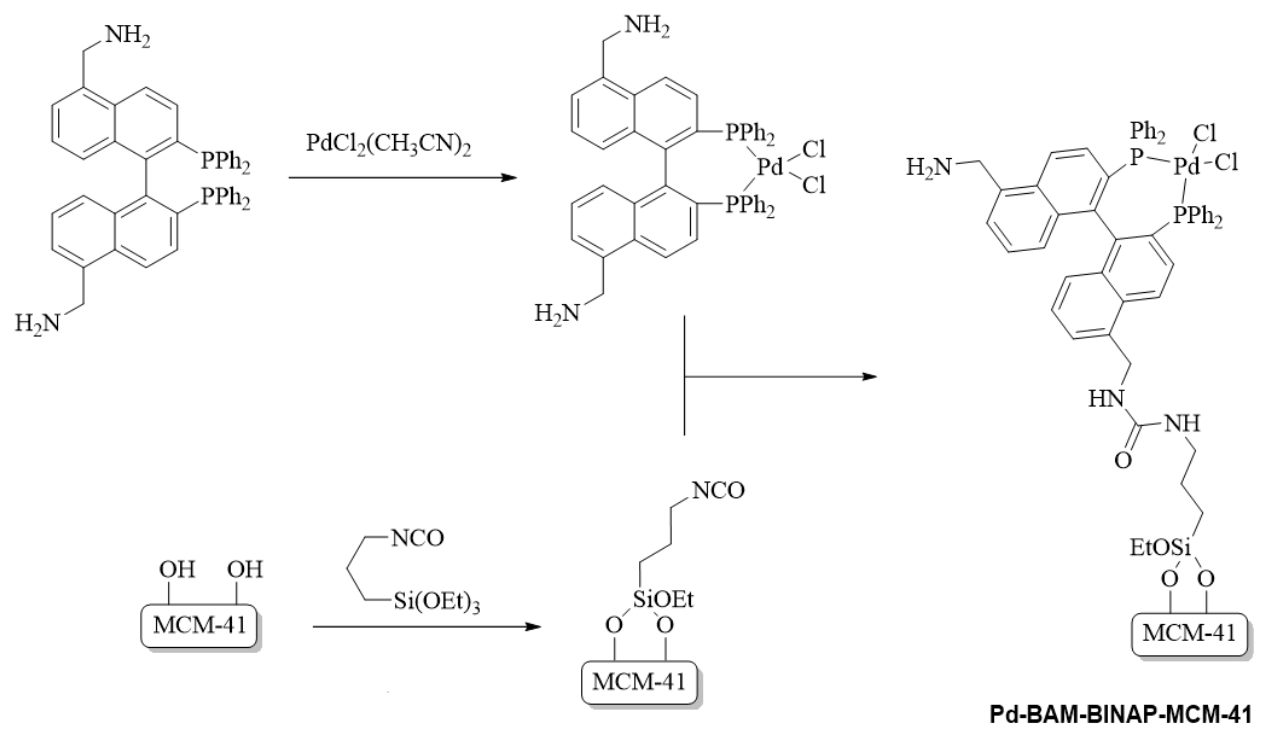

Figure 5.20: Schematic representation of the synthesis pathway to synthesize Pd-BAMBINAP-MCM-41.

The underlying assumption was that, given the low amount of linker incorporated onto the silica support, the isocyanate surface density would be low enough so that once one of the amine reacted with an isocyanate species, there wouldn't be another one in the close proximity to react with the other amine group. The rotational rigidity of the unsaturated platform molecule is also beneficial to this aim, as a platform backbone constituted by freely rotating saturated bonds, would increase the chances of bringing the second amine group close to a second isocyanate located on the surface of the support. $\mathrm{A}{ }^{13} \mathrm{C}$ CP/MAS NMR spectrum was recorded on Pd-BAM-BINAP-MCM-41 material (Fig. 5.21). In the region between 0 and $60 \mathrm{ppm}$, signals related to the aliphatic carbon atoms - from the propyl linker and from the methylene atoms linking the amines to the BINAP structure - are visible. In the $115-140$ ppm range, an intense composite signal due to the aromatic carbon atoms of the BINAP complex is observed. Lastly, a signal ascribed to the carbonyl carbon of the urea group is observed (marked with a dot in the figure) which confirms the good outcome of the tethering reaction. Further characterization is being carried out on the hybrid. This hybrid appears particularly interesting as BINAP-based catalysts prepared using enantiomerically pure ligands showed promising results for asymmetric catalysis in multiple studies. 


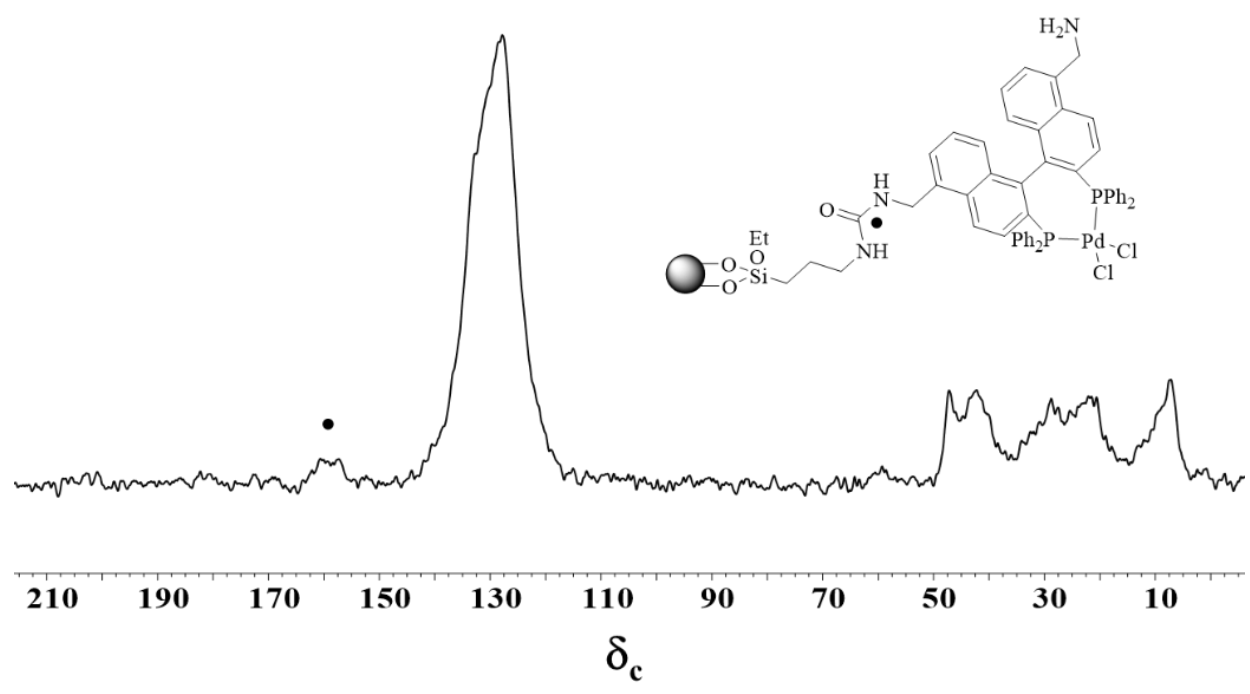

Figure 5.21: ${ }^{13} \mathrm{C}$ CP/MAS NMR spectrum of Pd-BAM-BINAP-MCM-41 material.

\subsubsection{Catalytic activity of the bifunctional Pd-Base catalysts}

To highlight the catalytic potential of the two bi-functional Pd-BisNHC@ $\mathrm{NH}_{2}-\mathrm{SiO}_{2}$ and Pd-BAM-BINAP-MCM-41 materials, the results of a catalytic test in a tandem reaction are reported below. Specifically, the process involves the Suzuki coupling between benzeneboronic acid and 4-bromobenzaldehyde followed by the aldol condensation between the product and acetone (Fig. 5.22). Due to confidentiality concerns, the conditions of the reaction are omitted.

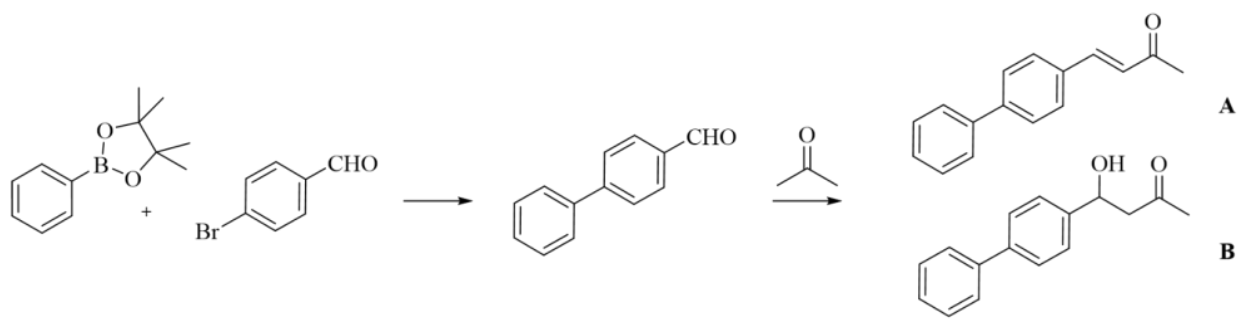

Figure 5.22: Schematic representation of the tandem reaction involving the Suzuki coupling between benzeneboronic acid and 4-bromobenzaldehyde followed by the aldol condensation of the product with acetone. 
Both hybrids show similar catalytic performances (Table. 5.3) with full conversion for the Suzuki step and a conversion of about $45 \%$ for the aldol step of the reaction. Interestingly, catalyst Pd-BisNHC@ $\mathrm{NH}_{2}-\mathrm{SiO}_{2}$ was more selective toward product A, while catalyst Pd-BAM-BINAP-MCM-41 showed higher selectivity toward product $\mathrm{B}$. This appears particularly notable as the functional group carrying out the reaction is the same, i.e. primary amine. Further catalytic testing and a deep characterization of the two hybrids might provide a rationale for this findings and are currently being carried out.

Table 5.3: Catalytic results in the tandem reaction.

\begin{tabular}{lcc}
\hline Catalyst & Conversion Suzuki step & Conversion aldol step \\
\hline Pd-BisNHC@ NH2-SiO & $100 \%$ & $43 \%(12 \% \mathrm{~A}, 31 \% \mathrm{~B})$ \\
Pd-BAM-BINAP-MCM-41 & $100 \%$ & $47 \%(27 \% \mathrm{~A}, 20 \% \mathrm{~B})$ \\
\hline
\end{tabular}




\subsection{Conclusions}

This Chapter focused on the synthesis of multi-functional hybrid catalysts in which base and metal sites were present. Different kind of catalysts, obtained with different synthetic approaches were described, in the attempt of giving on overview of some of the different possibilities available in the multi-step synthesis of multi-functional hybrids. This was done by outlining on-going studies that are being conducted by the partners of the MULTI2HYCAT consortium.

- Firstly, a catalyst featuring aminopropyl residues and palladium nanoparticles was prepared (named 1-Pd). Pure silica MCM-41 was chosen as support. Aminopropyl triethoxysilane was grafted onto the support and then, in a second step, palladium nanoparticles were formed in situ and deposited onto the amino-functionalized mesoporous silica. The pendant aminopropyl moieties were set to play two roles: (i) acting as base site in a catalytic process and (ii) stabilizing the newly formed palladium nanoparticles within the mesoporous channels by steric hindering and chemical interaction through the nitrogen lone electron pair.

- The catalyst was characterized using multiple techniques. X-ray diffractograms were recorded prior to and after palladium nanoparticles deposition, to ensure the structural integrity of the materials. Transmission electron micrographs were recorded on the bi-functional catalyst. Bright-field and dark-field images clearly showed the presence of nanoparticles with a fairly narrow dimensional distribution and without detecting the existence of metallic aggregates. This was quantified by measuring the size of 148 nanoparticles (in 6 images), resulting in a distribution centered at 2-2.5 $\mathrm{nm}$ of diameter, with a measured maximum measured value of $4.5 \mathrm{~nm}$. The average size of the particles appears to be the same as the internal pore channels of the MCM41 support. The most plausible interpretation of this finding is that nanoparticles forming within the channels, grow until reaching the pores dimension and are then stabilized by the presence of the aminopropyl moieties, that hinders their migration throughout the pores. $\mathrm{N}_{2}$ physisorption isotherms were recorded onto the two hybrids and compared with one recorded on the bare support, MCM-41. Isotherms and their analyses (carried out using BET and BJH methods) indicated a progressive reduction of surface area, pore volume and mean pore diameter. This last feature could be appreciated also by observing the isotherms, in which the inflexion point was shifted at lower relative pressures upon adding the aminopropyl fragments firstly, and then the palladium nanoparticles. ${ }^{13} \mathrm{C}$ CP/MAS NMR spectra were recorded on the two hybrids to confirm the preservation of the organic moieties during the synthetic steps.

- Hybrid 1-Pd catalyst was tested in a tandem reaction consisting of the aldol condensation between furfural and methyl isobutyl ketone, followed by hydrogenation of the aldol intermediate, resulting in three hydrogenated products: the desired 1-(furan-2-yl)-5-methylhexan-3-one, and the by-products 1-(furan-2-yl)-5-methylhex-1- 
en-3-ol and 1-(furan-2-yl)-5-methylhexan-3-ol. The influence of multiple variables was explored, such as the ratio between the two reactants, the amount of catalyst, $\mathrm{H}_{2}$ pressure, the presence of $\mathrm{H}_{2}$ during the first step of the reaction (aldol condensation) and temperature of the reactor. These insightful results pave the way for a more thorough optimization of the catalytic conditions that will be carried out in flow regime using a fixed-bed reactor.

- In the last Section of the Chapter, the synthesis of two hybrids featuring amine groups and a palladium complex are reported. The two catalyst represent two different approaches that are possible when two organic catalytic sites are to be introduced in a single solid material. In the first one, named Pd-BisNHC@ $@ \mathrm{NH}_{2}-\mathrm{SiO}_{2}$, the palladium complex was firstly prepared, by using a silylated ligand (derived from 1-methylimidazole). Specifically, each palladium atom was coordinated by two molecules of the silyl-derivative. The complex was labelled PdBisNHC. The active sites were incorporated onto commercial silica Ultrasil, by sequential grafting of aminopropyl triethoxysilane and of silyl-derivative PdBisNHC. The second one, on the other hand, was prepared from a molecular platform derived from BINAP, supplied by a partner of the MULTI2HYCAT consortium. The platform featured two amines groups, one of which was meant to serve as anchoring point to the support, while the other was meant to be left untouched and act as base site in a catalytic process. To achieve this, the catalyst was tethered to the surface of a MCM-41 support that was previously functionalized with low amount of surface isocyanatepropyl moieties. The potential of the two catalysts was showed in a tandem reaction involving a Suzuki coupling between benzeneboronic acid and 4-bromobenzaldehyde, followed by aldol condensation of the product with acetone.

The preliminary studies reported in this Chapter constitute important proof-of-concept of the potential that these multi-functional catalysts exhibit. The high catalytic activity of hybrid 1-Pd will be exploited in flow regime, with the scope of reaching a stady-state, possibly limiting or avoiding furfural polymerization within the mesopore channels. Results reported on the hybrids featuring base sites and palladium complex, on the other hand, open up to the possibility of using chiral ligands, which might allow to carry out asymmetric catalysis. The same approach could also be used to support complexes featuring other transition metals. These materials could find application in processes within the pharmaceutical industry and, more in general, could introduce new versatile strategies to approach the synthesis of multi-functional catalysts. All these aspects are currently being explored by the MULTI2HYCAT consortium. 


\subsection{References}

(1) Somorjal, G. A.; Carrazza, J. Ind. Eng. Chem. Fundam. 1986, 25, 63-69.

(2) Liu, L.; Corma, A. Chem. Rev. 2018, 118, 4981-5079.

(3) Grushin, V. V.; Alper, H. Chem. Rev. 1994, 94, 1047-1062.

(4) Polshettiwar, V.; Baruwati, B.; Varma, R. S. Green Chem. 2009, 11, 127-13.

(5) Polshettiwar, V.; Varma, R. S. Org. Biomol. Chem. 2009, 7, 37-40.

(6) Polshettiwar, V.; Len, C.; Fihri, A. Coord. Chem. Rev. 2009, 253, 2599-2626.

(7) Iwasawa, T.; Tokunaga, M.; Obora, Y.; Tsuji, Y. J. Am. Chem. Soc. 2004, 126, 65546555 .

(8) Petrosko, S. H.; Johnson, R.; White, H.; Mirkin, C. A. J. Am. Chem. Soc. 2016, 138, 7443-7445.

(9) Liu, C.; Cao, C.; Liu, J.; Wang, X.; Zhu, Y.; Song, W. J. Mater. Chem. A 2017, 5, 17464-17469.

(10) Lestini, E.; Blackman, L. D.; Zammit, C. M.; Chen, T.; Williams, R. J.; Inam, M.; Couturaud, B.; O’Reilly, R. K. Polym. Chem. 2018, 9, 820-823.

(11) Guerra, J.; Herrero, M. A. Nanoscale 2010, 2, 1390-1400.

(12) Gomez-Gualdron, D. A.; Dix, S. T.; Getman, R. B.; Snurr, R. Q. Phys. Chem. Chem. Phys. 2015, 17, 27596-27608.

(13) Gao, J.; Wu, S.; Tan, F.; Tian, H.; Liu, J.; Lu, G. Q. M. Prog. Nat. Sci. Mater. Int. 2018, 28, 242-245.

(14) Díaz-Sánchez, M.; Díaz-García, D.; Prashar, S.; Gómez-Ruiz, S. Environ. Chem. Lett. 2019, 17, 1585-1602.

(15) Barau, A.; Budarin, V.; Caragheorgheopol, A.; Luque, R.; MacQuarrie, D. J.; Prelle, A.; Teodorescu, V. S.; Zaharescu, M. Catal. Letters 2008, 124, 204-214.

(16) Li, P.; Liu, H.; Yu, Y.; Cao, C. Y.; Song, W. G. Chem. - An Asian J. 2013, 8, 24592465.

(17) Schmidt-Winkel, P.; Lukens, W. W.; Yang, P.; Margolese, D. I.; Lettow, J. S.; Ying, J. Y.; Stucky, G. D. Chem. Mater. 2000, 12, 686-696.

(18) Johnston, E. V.; Verho, O.; Kärkäs, M. D.; Shakeri, M.; Tai, C. W.; Palmgren, P.; Eriksson, K.; Oscarsson, S.; Bäckvall, J. E. Chem. - A Eur. J. 2012, 18, 12202-12206.

(19) Budroni, G.; Corma, A.; García, H.; Primo, A. J. Catal. 2007, 251, 345-353.

(20) Ma, Z.; Yang, H.; Qin, Y.; Hao, Y.; Li, G. J. Mol. Catal. A Chem. 2010, 331, 78-85.

(21) Kim, T. W.; Ryoo, R.; Kruk, M.; Gierszal, K. P.; Jaroniec, M.; Kamiya, S.; Terasaki, O. J. Phys. Chem. B 2004, 108, 11480-11489. 
(22) Yang, H.; Han, X.; Li, G.; Wang, Y. Green Chem. 2009, 11, 1184-1193.

(23) Biscoe, M. R.; Barder, T. E.; Buchwald, S. L. Angew. Chemie - Int. Ed. 2007, 46, 7232-7235.

(24) Del Pozo, C.; Corma, A.; Iglesias, M.; Sánchez, F. Green Chem. 2011, 13, 24712481.

(25) Bourissou, D.; Guerret, O.; Gabbaï, F. P.; Bertrand, G. Chem. Rev. 2000, 100, 3991.

(26) Hirai, K.; Itoh, T.; Tomioka, H. Chem. Rev. 2009, 109, 3275-3332.

(27) Fèvre, M.; Pinaud, J.; Gnanou, Y.; Vignolle, J.; Taton, D. Chem. Soc. Rev. 2013, 42, 2142-2172.

(28) Enders, D.; Niemeier, O.; Henseler, A. Chem. Rev. 2007, 107, 5606-5655.

(29) Singh, R.; Kissling, R. M.; Letellier, M. A.; Nolan, S. P. J. Org. Chem. 2004, 69, 209-212.

(30) Marion, N.; Díez-González, S.; Nolan, S. P. Angew. Chemie - Int. Ed. 2007, 46, 2988-3000.

(31) Fortman, G. C.; Nolan, S. P. Chem. Soc. Rev. 2011, 40, 5151-5169.

(32) Doddi, A.; Peters, M.; Tamm, M. Chem. Rev. 2019, 119, 6994-7112.

(33) Niknam, E.; Panahi, F.; Khalafi-Nezhad, A. Appl. Organomet. Chem. 2020, 34, 115.

(34) Mondal, M.; Joji, J.; Choudhury, J. Chem. Commun. 2017, 53, 3185-3188.

(35) Polshettiwar, V.; Varma, R. S. Tetrahedron 2008, 64, 4637-4643.

(36) Noyori, R.; Nobel Lecture 2001 Adv. Synth. Catal. 2003, 345, 15-32.

(37) Yang, P.; Zheng, C.; Nie, Y. H.; You, S. L. Chem. Sci. 2020, 11, 6830-6835.

(38) Uno, H.; Punna, N.; Tokunaga, E.; Shiro, M.; Shibata, N. Angew. Chemie - Int. Ed. 2020, 59, 8187-8194.

(39) Ma, B. De; Du, S. H.; Wang, Y.; Ou, X. M.; Huang, M. Z.; Wang, L. X.; Wang, X. G. Tetrahedron Asymmetry 2017, 28, 47-53.

(40) Li, J.; Smith, D.; Krishnananthan, S.; Mathur, A. Org. Process Res. Dev. 2020, 24, 454-458..

(41) Zhang, L.; Huang, X.; Zhen, S.; Zhao, J.; Li, H.; Yuan, B.; Yang, G. Org. Biomol. Chem. 2017, 15, 6306-6309.

(42) Xu, Z.; Yu, X.; Sang, X.; Wang, D. Green Chem. 2018, 20, 2571-2577.

(43) Zhang, Q.; Wang, H.; Hu, W.; Xu, X.; Xu, Z.; Chen, C.; Wang, D. J. Chem. Technol. Biotechnol. 2020, 95, 2027-2033. 
(44) Reimann, S.; Grunwaldt, J. D.; Mallat, T.; Baiker, A. Chem. - A Eur. J. 2010, 16, 9658-9668.

(45) Climent, M. J.; Corma, A.; Iborra, S. Green Chem. 2014, 16, 516-547.

(46) Wang, H.; Yang, B.; Zhang, Q.; Zhu, W. Renew. Sustain. Energy Rev. 2020, 120, 109612.

(47) Deng, Q.; Xu, J.; Han, P.; Pan, L.; Wang, L.; Zhang, X.; Zou, J. J. Fuel Process. Technol. 2016, 148, 361-366.

(48) Xing, R.; Subrahmanyam, A. V.; Olcay, H.; Qi, W.; van Walsum, G. P.; Pendse, H.; Huber, G. W. Green Chem. 2010, 12, 1933-1946.

(49) Yang, J.; Li, N.; Li, G.; Wang, W.; Wang, A.; Wang, X.; Cong, Y.; Zhang, T. ChemSusChem 2013, 6, 1149-1152.

(50) Chang, H.; Motagamwala, A. H.; Huber, G. W.; Dumesic, J. A. Green Chem. 2019, 21, 5532-5540.

(51) Jiang, S.; Ramdani, W.; Muller, E.; Ma, C.; Pera-Titus, M.; Jerôme, F.; De Oliveira Vigiera, K. ChemSusChem 2020, 13, 1699-1704.

(52) Jiang, S.; Ma, C.; Muller, E.; Pera-Titus, M.; Jérôme, F.; De Oliveira Vigier, K. ACS Catal. 2019, 9, 8893-8902.

(53) Liang, G.; Wang, A.; Zhao, X.; Lei, N.; Zhang, T. Green Chem. 2016, 18, 3430 3438 .

(54) Sádaba, I.; Ojeda, M.; Mariscal, R.; Fierro, J. L. G.; Granados, M. L. Appl. Catal. B Environ. 2011, 101, 638-648.

(55) Shen, W.; Tompsett, G. A.; Hammond, K. D.; Xing, R.; Dogan, F.; Grey, C. P.; Conner, W. C.; Auerbach, S. M.; Huber, G. W. Appl. Catal. A Gen. 2011, 392, $57-$ 68.

(56) Kikhtyanin, O.; Ganjkhanlou, Y.; Kubička, D.; Bulánek, R.; Čejka, J. Appl. Catal. A Gen. 2018, 549, 8-18.

(57) Li, M.; Xu, X.; Gong, Y.; Wei, Z.; Hou, Z.; Li, H.; Wang, Y. Green Chem. 2014, 16, 4371-4377.

(58) Moteki, T.; Koga, Y.; Ogura, M. J. Catal. 2019, 378, 131-139.

(59) Zhong, R.; Lindhorst, A. C.; Groche, F. J.; Kühn, F. E. Chem. Rev. 2017, 117, 1970 2058. 

Chapter 6

\section{General Conclusions}



Below, general conclusions of this Doctoral Thesis are reported.

- Several hybrid mono- and multi-functional catalysts have been prepared through various synthetic approaches, characterized and tested, in collaboration with academic and industrial partners of the MULTI2HYCAT project.

- Mono-functional hybrid catalysts featuring aryl-sulfonic acid moieties were prepared. In one of the hybrids ( $\mathrm{HSO}_{3}-\mathrm{NOS}-\mathrm{F}$ ), the aromatic ring featured a fluorine substituent in position 2, 3, 5, 6, while in the other hybrid ( $\left.\mathrm{HSO}_{3}-\mathrm{NOS}-\mathrm{H}\right)$, those position featured a hydrogen atom. This was done to study the effect of the substituents of the aromatic ring on the catalytic activity of silica-supported sulfonic acids.

- The two acid hybrids were characterized, using an array of techniques. The hybrids showed textural properties typical of mesoporous materials with a pore size distribution centered at $35 \AA$ and a specific surface area between $700-800 \mathrm{~m}^{2} / \mathrm{g}$. Both hybrids were shown to be stable up to $200-250{ }^{\circ} \mathrm{C}$. FTIR and ${ }^{13} \mathrm{C}$ solid state CP/MAS NMR spectroscopies allowed to observe signals related to the presence of key functional groups (e.g. sulfonic groups, urea groups, aromatic ring), confirming the presence of the desired structures in both materials, beyond doubts. ${ }^{1} \mathrm{H}$ solid state MAS NMR allowed to study the proton speciation within the catalysts, showing signals consistent with aliphatic and aromatic protons from the organic moieties, as well as the presence of silanols, both isolated and interacting with each other through $\mathrm{H}$ bond.

- For the first time, probe molecules were adsorbed onto the silica-based hybrids and their interactions were studied by spectroscopic techniques. The probe molecules implemented were carbon monoxide (FTIR), ammonia (FTIR, NMR) and trimethylphosphine oxide (NMR). These studies allowed obtaining insightful information on the interaction occurring between pendant organic moieties and support's surfaces, as well as information on the acidic properties of the hybrids.

- The acid hybrid catalysts were tested in the acetal formation reaction between ethylene glycol and benzaldehyde. Both catalysts showed remarkable activity and reusability. The fluorinated catalyst showed a better preservation of the catalytic activity over multiple cycles, compared to the non-fluorinated one. The latter was also tested in other acetalization reactions, highlighting the potential of these hybrid acid catalysts.

- A hydrophobic version of the organic-inorganic catalysts was prepared. Surface silanols groups were reacted with hexamethyl disilazane, through grafting methods. The catalysts were characterized showing reduced textural properties and spectroscopic features that confirmed the incorporation of trimethyl-silyl species onto the surface of the hybrids. Both hybrid catalysts showed good catalytic performances in the acetal formation reaction between benzaldehyde and ethylene glycol, although not as good as the hydrophilic hybrids. 
- Six hybrid catalysts featuring base functional groups were prepared by grafting silylderivatives onto MCM-41 support. These silyl-derivatives, featuring base active sites and a highly reactive terminal trialkoxysilyl group, were either bought from commercial sources or synthesized starting from commercially available precursors. Three of the hybrids featured primary or secondary amines as base sites (non-ionic catalysts), while three others featured ammonium hydroxide species derived from $\mathrm{N}$-heterocycles (ionic catalysts). Mildly acidic silanol groups present on the surface of silica supports are known to co-catalyze reactions in synergy with pendant organocatalysts featuring base active sites. A passivated version of this type of hybrid catalys was also prepared by capping the surface silanol with hexamethyl disilazane (HMDS), in order to directly evaluate the effect of surface silanols on the catalytic activity of the materials.

- The base catalysts were thoroughly characterized by means of a multi-technique approach. Structural and textural properties typical of mesoporous solids derived from MCM-41 support were observed. Elemental analysis indicated that roughly the same amounts of active site were incorporated in each catalyst. Thermogravimetric analyses provided useful information on the thermal stability of the hybrid catalysts and specifically of the pendant organic moieties. FTIR, ${ }^{13} \mathrm{C}$ and ${ }^{29} \mathrm{Si}$ NMR spectroscopies confirmed the integrity of the organic species after the grafting, confirming that the target hybrid base catalysts were achieved.

- The hybrid base catalysts were tested in Knovenagel condensation reactions of benzaldehyde and substrates featuring different $\mathrm{pKa}$, i.e. ethyl cyanoacetate $(\mathrm{pKa} \approx 9)$, ethyl acetoacetate $(\mathrm{pKa} \approx 11)$ and diethyl malonate $(\mathrm{pKa} \approx 13)$. Non-ionic catalysts showed superior catalytic activity, compared to ionic catalysts. Specifically, the most performing hybrid featured aminopropyl moieties as active sites. The hydrophobic version of this catalyst performed much worse than its hydrophilic counterpart, highlighting the key role played by surface silanol in cooperatively catalyze the reaction. Recycle tests were carried out, indicating stable catalytic activity over three catalytic cycles. An imine mechanism was proposed for this reaction and alternative reaction pathways were also discussed.

- The hybrid base catalysts were also tested in the Michael addition of ethyl 2-oxocyclopentane 2-carboxylate (EtOCC) and methyl vinyl ketone (MVK). In contrast with what was observed for the Knoevenagel condensation reaction, ionic catalysts featured much higher activity than non-ionic catalysts. An enolate mechanism was proposed for this reaction. Differences in activity among ionic catalysts was explained considering the differences between $\mathrm{N}$-heterocycles and how they affected their ability in stabilizing a positive charge. Hydrophobic 1-HMDS performed worse than its hydrophilic counterpart, for this reaction as well.

- The base catalysts were finally tested in the reaction between furfural and methyl isobutyl ketone. The reaction was selected upon the need of the industrial partners 
of the MULTI2HYCAT project and represents the first step of a tandem reaction, featuring as second step the hydrogenation of the newly formed C-C double bond. The hybrid catalyst featuring aminopropyl moieties showed the highest catalytic activity with $77 \%$ yield of the desired product after $24 \mathrm{~h}$. The other five catalysts featured a yield after $24 \mathrm{~h}$ below $20 \%$. An imine mechanism was proposed for this reaction.

- A versatile strategy for synthesizing silica-supported P1 phosphazenium hydroxide moieties was described. The synthesis process involved the synthesis of a silyl-derivative featuring P1 functional groups, a grafting step and an ion exchange with a tetramethylammonium hydroxide solution. Structural and textural properties confirmed the preservation of the M41S structure throughout the process; ${ }^{31} \mathrm{P},{ }^{13} \mathrm{C}$ and ${ }^{29} \mathrm{Si}$ solid state MAS NMR showed signals consistent with the presence of the target organic moieties. This work was intended to be a proof of concept, as the silyl-derivative featuring the phosphazene group is relatively stable and can be used for the future design of mono- or multi-functional effective and active catalysts.

- Building upon the results obtained for MCM-41-supported base catalysts, a multifunctional catalyst featuring aminopropyl residues and palladium nanoparticles was prepared. Firstly, aminopropyl-functionalized MCM-41 was synthesized and, in a subsequent step, palladium nanoparticles were formed in situ and deposited onto it. The pendant aminopropyl moieties of this hybrid catalyst were set to play two roles: (i) acting as base site in a catalytic process and (ii) stabilizing the newly formed palladium nanoparticles within the mesoporous channels by steric hindering and chemical interaction through the nitrogen lone electron pair.

- The multi-functional hybrid catalyst was characterized using multiple techniques. Transmission electron micrographs were recorded on the multi-functional catalyst, indicating the presence of nanoparticles with a narrow dimensional distribution, centered at 2-2.5 nm, without detecting the existence of metallic aggregates. The average size of the particles appears to be the same as the internal pore channels of the MCM-41 support, which suggests their preferential location within the pore channels. This was also confirmed by $\mathrm{N}_{2}$ physisorption analysis of the hybrid which indicated a progressive reduction of surface area, pore volume and mean pore diameter, upon functionalization with aminopropyl fragments and palladium nanoparticles. ${ }^{13} \mathrm{C} \mathrm{CP} / \mathrm{MAS}$ NMR spectra were recorded to confirm the preservation of the organic moieties during the synthetic steps.

- The multi-functional catalyst featuring aminopropyl moieties and palladium nanoparticles was tested in a tandem reaction consisting of the aldol condensation between furfural and methyl isobutyl ketone, followed by hydrogenation of the aldol intermediate, resulting in three hydrogenated products. The influence of several variables was explored, such as the temperature of the reactor, the ratio between the two reactants, the amount of catalyst, $\mathrm{H}_{2}$ pressure and the presence of $\mathrm{H}_{2}$ during the first 
step of the reaction (aldol condensation). These results pave the way for a more thorough optimization of the catalytic conditions that will be carried out in flow conditions using a fixed-bed reaction.

- The synthesis of two hybrids featuring amine groups and a palladium complex was also described. Pd-BisNHC@ $\mathrm{NH}_{2}-\mathrm{SiO}_{2}$ was obtained from a palladium complex, previously prepared using a silylated ligand (derived from 1-methylimidazole). The silylated complex was named PdBisNHC. The active sites were supported onto commercial silica Ultrasil, by sequential grafting of aminopropyl triethoxysilane and of silyl-derivative PdBisNHC.

- Another catalyst was prepared starting from a molecular platform derived from BINAP that was supplied by a partner of the MULTI2HYCAT consortium. The platform featured two amines groups one of which was meant to serve as anchoring point to the support, while the other was meant to be left untouched and act as base site in a catalytic process. To achieve this, a strategy was developed involving, firstly, the synthesis of a MCM-41-based hybrid functionalized with isocyanatepropyl moieties and then a tethering reaction between the pendant isocyanate groups and one of the amine groups of the platform molecule. The catalyst was named PdBAM-BINAP-MCM-41.

- The potential of multi-functional hybrid catalysts Pd-BisNHC@ $\mathrm{NH}_{2}-\mathrm{SiO}_{2}$ and PdBAM-BINAP-MCM-41 was showed in a tandem reaction involving a Suzuki coupling between benzeneboronic acid and 4-bromobenzaldehyde, followed by aldol condensation of the product with acetone. 


Appendix 1

\section{Characterization Techniques}



Below, the characterization techniques implemented in this Doctoral Thesis are described.

\section{X-Ray powder diffraction (XRD)}

It is a characterization technique that allows obtaining information on the crystalline structure of a material. Crystalline frameworks can coherently diffract X-ray photons. Each specific family of parallel crystalline planes scatters photons at a specific angle, according to Bragg's law:

$$
n \lambda=2 d_{(h k l)} \sin \theta
$$

Where $n$ is a whole number, $\lambda$ is the wavelength of the beam-out, $d$ is the distance between planes and $\theta$ is the angle between the beam-in and the crystalline planes. The photons are diffracted at an angle $\theta$, respect to the family of crystalline planes. Therefore, the sample is irradiated with X-ray photons of a specific wavelength $\lambda$, and the intensity of diffracted photons is collected as a function of the $2 \theta$ angle. The pattern generated is typical of a given crystalline structure.

XRD measurements were carried out using a Philips X'PERT diffractometer equipped with a proportional detector and a secondary graphite monochromator. Data were collected stepwise in the $2^{\circ} \leq 2 \theta \leq 20^{\circ}$ angular region: $0.02^{\circ} 2 \theta$ steps, $20 \mathrm{~s} /$ step accumulation time, beam-in was produced from $\mathrm{Cu} \mathrm{K \alpha}(\lambda=1.54178 \AA) .{ }^{1}$

\section{Thermogravimetric analysis}

The technique allows to measure weight losses occurring at a sample during a heating ramp in a certain temperature interval (TGA). The differential analysis of the thermograms (DTA) can provide information on the nature of the process, i.e. if it is endo- or exothermic. This technique is crucial in the study of hybrid catalysts, as it allows to gather knowledge on the thermal stability of the organic fragments. Moreover, it can indirectly confirm the presence of the desired linking bond, by comparison with other studies reported in the scientific literature.

Thermogravimetric analyses (TGA/DTA) of materials reported in Chapter 2 were performed under argon flow $\left(100 \mathrm{~mL} \mathrm{~min}^{-1}\right)$ with a SETSYS Evolution TGA-DTA/DSC thermobalance. Thermogravimetric analyses (TGA/DTA) reported in Chapter 3 were conducted in air stream with a Mettler Toledo TGA/SDTA $851 E$ analyzer. The heating ramp was set from 40 to $800 / 1000{ }^{\circ} \mathrm{C}$ at $5{ }^{\circ} \mathrm{C} \mathrm{min}^{-1}$.

\section{Elemental analysis}

The organic content in the hybrid catalysts was measured by elemental analysis using a Carlo Erba 1106 analyzer. Typically, sample are treated at high temperature in presence of oxygen, which favors the combustion of the organic fragments and consequentially induces the release of combustion products (gases). The amounts of gases are quantified 
using a thermal conductivity detector giving signals that are proportional to the quantities of each element in the composition of the sample analyzed.

\section{$\mathbf{N}_{2}$ physisorption analysis at $77 \mathrm{~K}$}

Nitrogen adsorption is a widely used techniques to evaluate the textural properties of solid materials. Initially the sample is pre-treated in a glass vessel to removed physisorbed moieties (e.g. water). Then, dosages of $\mathrm{N}_{2}$ are adsorbed onto the sample in the $\mathrm{p} / \mathrm{p}_{0}$ range between 0 and 1 . Specifically, for each point of the isotherm plot, the instrument attempts to slightly increase the pressure within the glass vessel, by dosing $\mathrm{N}_{2}$ until reaching a stable pressure value. The amount of gas needed to reach such stable value will be different for each point of the isotherm and for each sample, as a function of its textural properties.

Preliminary insights can be drawn from the isotherms alone. However, it is common to analyze the isotherms with various methods to gain more precise information about surface area, pore size and pore volume of the materials. Below those implemented in this Doctoral Thesis are briefly described.

Brauner Emmet Teller (BET): The theory is an extension of the Langmuir theory, which deal with monolayer molecular adsorption. The multilayer adsorption contemplated by the BET method lies upon the following hypotheses: (i) gas molecules physically adsorb on a solid in layers infinitely; (ii) gas molecules only interact with adjacent layers and the Langmuir theory can be applied to each layer; (iii) the enthalpy of adsorption for the first layer is constant and greater than the second; (iv) the enthalpy of adsorption for the second (and higher) layers is the same as the enthalpy of liquefaction. The equation that derives is the following:

$$
\frac{1}{v\left[\left(\frac{p_{0}}{p}\right)-1\right]}=\frac{c-1}{v_{m} c}\left(\frac{p}{p_{0}}\right)+\frac{1}{v_{m} c}
$$

where $p$ and $p_{0}$ are the equilibrium and the saturation pressure of adsorbate at the temperature of adsorption, $v$ is the adsorbed gas quantity, $v_{m}$ is the monolayer adsorbed gas quantity and $c$ is the BET constant

$$
c=\exp \left(\frac{E_{1}-E_{L}}{R T}\right)
$$

where $E_{l}$ is the heat of adsorption for the first layer, and $E_{L}$ is that for the second and higher layers (equal to the heat of liquefaction). The graphic representation of the adsorption data allows to identify the volume of gas that is needed to complete the monolayer and, accordingly, to estimate the specific surface area in $\mathrm{m}^{2} / \mathrm{g}$ through the following equation:

$$
S S A_{B E T}=\frac{v_{m} N_{A} A_{m}}{M}
$$


Where $A_{m}$ is the area occupied by a $\mathrm{N}_{2}$ molecule, $N_{A}$ is Avogadro's constant and $M$ is the volume occupied by a molecule of $\mathrm{N}_{2}$ under ideal conditions.

Barrett, Joyner, Helenda (BJH): It is a modified version of the Kelvin equation. Usually implemented on the desorption branch of the isotherm, it correlates the volume of gas adsorbed onto the material at a certain pressure with the pore volume due to pores of a specific size, ultimately allowing to estimate a pore size distribution and a porous volume.

Non-local density functional theory (NLDFT): this method uses a classical fluid density functional theory to construct the adsorption isotherms in ideal pore geometries (e.g., $\mathrm{N}_{2}$ adsorption in the cylindrical-pore model at $77 \mathrm{~K}$ ). The pore size distribution can be obtained by solving an adsorption integral equation. This task is carried out by using a software and several variables need to be set before proceeding, such as nature of the adsorbant/adsorbate and the shape of the pores.

It should be highlighted that BET, BJH and NLDFT methods are useful tools that provide useful information but are, nevertheless, mathematical analyses of the experimental data (i.e. isotherm plot). They lie on assumptions and can underestimate/overestimate the textural properties of the samples. ${ }^{2,3}$

Isotherms were measured at $77 \mathrm{~K}$ with a Micromeritics ASAP 2010 volumetric adsorption analyzer. Prior to the measurement, the sample was outgassed for 12 hours at $80^{\circ} \mathrm{C}$. The BET specific surface area was calculated from the nitrogen adsorption data in the relative pressure range from 0.04 to 0.2 .

\section{Compositional analysis}

The chemical composition of the materials was determined by inductively coupled plasma atomic emission spectroscopy (ICP-OES). This technique relies on the excitation of the atoms that are present in a solution. The decay of the atom to its fundamental state cause an emission that is characteristic for each atom and the concentration of each signal will be proportional to the concentration of that element in the solution. This feature allows for quantitative analysis of the samples, carried out by linear regression from a calibration curve. Prior to the analysis, each sample was dissolved in a solution containing $\mathrm{HF}$ and $\mathrm{HNO}_{3}$. The analyses were performed using a VARIAN 715-ES instrument.

\section{Electrospray ionization time-of-fly mass spectrometry (ESI-TOF MS)}

Time-of-flight mass spectrometry (TOF MS) is a mass spectrometry method in which the mass/charge ratio of an ion $(\mathrm{m} / \mathrm{z})$ is determined via a time-of-flight measurement. For that, ions are accelerated by an electric field of known magnitude. This acceleration results in all ions featuring the same charge having the same kinetic energy. Consequentially, the velocity $v$ of each ion depends on the mass-to-charge ratio (as $E_{k}=1 / 2 m v^{2}$ ). Heavier ions feature lower speed, while lighter ions reach higher speed (provided that they have the same charge). The time that it takes for each ion to reach a detector at a 
known distance is measured and is dependent on the velocity of the ion, and therefore on its $\mathrm{m} / \mathrm{z}$. From this ratio and known experimental parameters, one can identify the ion. ESI-TOF MS measurements were performed using a Acquity XevoQToF spectrometer.

\section{Fourier-transform infrared spectroscopy (FTIR)}

The technique based upon the adsorption of infrared photons of different wavelength by a sample. The adsorption of infrared photons of specific wavelength activates vibrational modes of specific bonds, if such modes are IR-active, i.e. if the vibration causes a change in the dipolar momentum. The IR spectrum of a sample, plotting the intensity of adsorption as a function of the wavelength (or wave number) can indicate the presence of certain functional groups. Signals related to stretching modes will usually be found at higher wave numbers, as such modes require more energy to be activated. Conversely, signals related to bending modes will be found at lower wave numbers. The technique is especially useful for the analysis of surfaces and to study the acid-base properties of materials.

The FTIR spectra reported in Chapter 2 were collected from self-supporting pellets under vacuum conditions (residual pressure $<10^{-5} \mathrm{mbar}$ ) using a Bruker Equinox 55 spectrometer equipped with a pyroelectric detector (DTGS type) with a resolution of $4 \mathrm{~cm}^{-1}$. Probe molecules were adsorbed on the hybrid materials (previously outgassed at $150{ }^{\circ} \mathrm{C}$ ) by using a vacuum line, connected to a flask containing the probe molecule in gas phase.

Spectra reported in Chapter 3 were collected using a Nicolet 710 FTIR spectrometer. Samples were outgassed at $150{ }^{\circ} \mathrm{C}$ before the analysis.

\section{Nuclear magnetic resonance spectroscopy (NMR)}

This powerful spectroscopic technique can provide information on the chemical surrounding of the atoms that compose the material. It is based on the interactions between a magnetic field and the spin angular momentum of a given nuclei. Typically, the sample is placed in a magnetic field and the NMR signal is produced by excitation of the nuclei sample with radio waves into nuclear magnetic resonance, which is detected with sensitive radio receivers. The intramolecular magnetic field around an atom in a molecule changes the resonance frequency, giving access to details of the electronic structure of a molecule and its individual functional groups. The different electron density surrounding each nucleus will cause different shielding of the magnetic field which will induce differences in the resonance frequencies. Such differences are quantified in terms of chemical shift $(\delta)$. NMR spectroscopy is the ultimate method to identify monomolecular organic compounds.

Several interactions can occur, influencing the spectral profile. J-coupling, for example, arises from the interaction of different spin states through the chemical bonds of a molecule and results in the splitting of NMR signals. These features can provide important information on the sample and, together with the chemical shift, can allow to understand 
the fine-structure of the sample. Another important interaction is the dipolar coupling. In the liquid phase, given the random orientation of the molecules in a solution, this interaction is averaged to zero. However, it is present when spectra are recorded on solid samples, with detrimental effect on the output of the analysis. To avoid this problem, in a solid state spectrometer, the sample is rotated at 54 ${ }^{\circ} 44^{\prime}$ (magic angle spinning, MAS), respect the magnetic field. In doing so, dipolar couplings are averaged to zero.

Spectra reported in Chapter 2 were measured on a Bruker Advance III 500 spectrometer and a wide bore 11.7 Tesla magnet with operational frequencies for ${ }^{1} \mathrm{H},{ }^{13} \mathrm{C},{ }^{31} \mathrm{P}$ and ${ }^{29} \mathrm{Si}$ of 500.13, 125.77, 202.47 and $99.35 \mathrm{MHz}$, respectively. A $4 \mathrm{~mm}$ triple resonance probe with MAS was employed for all the experiments. The samples were packed on a zirconia rotor and spun at a MAS rate between 10 and $15 \mathrm{kHz}$. The magnitude of radio frequency (RF) fields was 100 and $42 \mathrm{kHz}$ for ${ }^{1} \mathrm{H}$ and ${ }^{29} \mathrm{Si}$, respectively. The relaxation delay between accumulations was 2 and $2.5 \mathrm{~s}$. For ${ }^{13} \mathrm{C}$ and ${ }^{29} \mathrm{Si}$ cross polarization (CP) MAS experiments, RF fields of 55 and $28 \mathrm{kHz}$ were used for initial proton excitation and decoupling, respectively. During the CP period the ${ }^{1} \mathrm{H}$ RF field was ramped using 100 increments, whereas the ${ }^{13} \mathrm{C} /{ }^{29} \mathrm{Si}$ RF fields were maintained at a constant level. During the acquisition, the protons are decoupled from the carbon atoms/silicon atoms by using a two-pulse phase-modulated (TPPM) decoupling scheme. A moderate ramped RF field of $62 \mathrm{kHz}$ was used for spin locking, while the carbon/silicon RF field was matched to obtain the optimal signal. A rotor-synchronized spin-echo sequence $(\pi / 2-\tau-\pi-\tau-$ acquisition) was also applied to record the ${ }^{1} \mathrm{H}$ NMR spectra with a delay time $\tau$ of $2 \mathrm{~ms}$. The delay time was chosen as an optimized compromise between the signal decay owing to relaxation and the resolution gain owing to longer delay times. ${ }^{31}$ P MAS NMR spectra were recorded using high power proton decoupling and the RF field for the 90-degree pulse was $83 \mathrm{kHz}$. All chemical shifts are reported using the $\delta$ scale and are externally referenced to tetramethylsilane (TMS) at $0 \mathrm{ppm}$ for ${ }^{1} \mathrm{H},{ }^{13} \mathrm{C}$ and ${ }^{29} \mathrm{Si}$. Ammonium dihydrogen phosphate $(1 \mathrm{ppm})$ was used as a standard for the ${ }^{31} \mathrm{P}$ nucleus. Prior to the adsorption of TMPO probe molecules, the catalysts were packed onto a $4 \mathrm{~mm}$ zirconia rotor and outgassed at $180^{\circ} \mathrm{C}$ for 90 minutes. After that, TMPO dissolved in anhydrous dichloromethane was added to the rotor and rapidly closed with a cap and left for adsorption for one hour. Later, the rotor was opened and attached back to the high vacuum line for the complete removal of dichloromethane at room temperature. Subsequently, the rotor was closed with a zirconia cap and heated to $160{ }^{\circ} \mathrm{C}$ for 30 minutes followed by submission to NMR spectral recording.

Solid state MAS NMR spectra reported in Chapter 3 were recorded at room temperature in a Bruker AV-400 spectrometer. The single pulse ${ }^{29} \mathrm{Si}$ spectra were recorded at 79.5 $\mathrm{MHz}$ with a $7 \mathrm{~mm}$ Bruker BL-7 probe, using pulses of $3.5 \mu$ s corresponding to a flip angle of $3 / 4 \pi$ radians and a recycle delay of $240 \mathrm{~s} .{ }^{1} \mathrm{H}$ to ${ }^{13} \mathrm{C}$ cross polarization (CP) spectra were acquired by using a $90^{\circ}$ pulse for ${ }^{1} \mathrm{H}$ of $5 \mu \mathrm{s}$, a contact time of $5 \mathrm{~ms}$, and a recycle of $3 \mathrm{~ms} .{ }^{13} \mathrm{C}$ spectra were recorded with a $7 \mathrm{~mm}$ Bruker BL-7 probe and at a sample spinning rate of $5 \mathrm{kHz} .{ }^{29} \mathrm{Si}$ and ${ }^{13} \mathrm{C}$ were referred to tetramethylsilane and adamantine, respectively. 
Liquid state ${ }^{13} \mathrm{C}$ NMR spectra reported throughout the Doctoral Thesis were recorded at $75 \mathrm{MHz}$ using a Bruker AMX300 instrument. Chemical shifts were reported in ppm and are referenced to the appropriate residual solvent peak.

\section{Transmission electron microscopy (TEM)}

It is a microscopy technique in which a beam of electrons is transmitted through a specimen to form an image. The electrons composing the beam-in can pass through the samples or be diffracted as a function of (i) the width of the portion of the sample that is traversing and (ii) the type of atom that they encounter along the way. Among the many use of this technique, it allows to obtain information on the size of the crystallites, on the presence of mesopores (if ordered) and on the presence of phases of different chemical composition (guest) within the material (host). The latter is possible if the atom number of the element composing the guest phase greatly differs from that of the host phase. The prime example of this implementation of the technique is the detection of metal nanoparticles supported onto silica or alumina.

Transmission electron micrographs were acquired with a JEOL JEM2100 F electron microscope operating at $200 \mathrm{keV}$. The samples were treated through the dispersion of the powders onto carbon copper grids. 
Appendix 1

\section{References}

(1) Jenkis, R; Snyder, R. Introduction to X-Ray Powder Diffractometry, 2012, Wiley.

(2) Kupgan, G.; Liyana-Arachchi, T. P.; Colina, C. M. Langmuir 2017, 33, 11138 11145 .

(3) Lowell, S.; Shields, J. E.; Thomas, M. A.. Characterization of Porous Solids and Powders: Surface Area, Pore Size and Density, 2006, Springer Science \& Business Media.

(4) Brunauer, S.; Emmett, P. H.; Teller, E., JACS 1938, 60, 309-319. 

Appendix 2

\section{List of Figures and Tables}





\section{List of Figures}

Figure 1.1: STEREOVIEW OF THE ACTIVE SITE OF GLUCOSE OXIDASE WITH THE MODELLED SUBSTRATE $\beta$-DGLUCOSE (ADAPTED FROM REF. 4). B: THE STRUCTURE OF T-VALEROLACTONE WITHIN ZEOLITE PORES AS OBTAINED USING IN SITU X-RAY DIFFRACTION DATA (ADAPTED FROM REF. 5). C: SINGLE PT ATOMS BOUNDED TO N- RICH OR S- RICH REGIONS IN DOPED CARBONACEOUS SUPPORTS (ADAPTED FROM REF. 10)......

FIGURE 1.2: SCHEME OF THE MAIN CHEMICAL ROUTES FOR THE SYNTHESIS OF ORGANIC-INORGANIC HYBRIDS. ADAPTED FROM REF. 11

FIGURE 1.3 A: SCHEMATIC REPRESENTATION OF THE POROUS STRUCTURE OF A PHENYL MODIFIED SILICA MATRIX DOPED WITH RHODAMINE 6G MOLECULES (ADAPTED FROM REF. 13). B: STRUCTURE OF PVPSILICA HYBRID MATERIAL (ADAPTED FROM REF. 14).

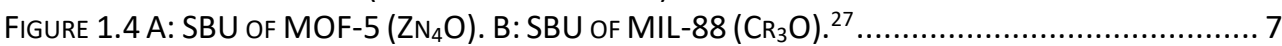

FIGURE 1.5: VARIOUS CARBOXYLIC ACID LINKERS USED FOR MOF SYNTHESIS AND A COMMON NITROGEN LINKER GROUP BIPY. ${ }^{29}$

FIGURE 1.6: DIFFERENT ACTIVE SITES AVAILABLE IN MOFS RESPONSIBLE FOR CATALYSIS. ${ }^{48} \ldots \ldots \ldots \ldots \ldots \ldots . . . \ldots$

FiguRE 1.7: STRUCTURES OF MESOPOROUS M41S MATERIALS: MCM-41 (A), MCM-48 (B) AND MCM$50(\mathrm{C}){ }^{49}$

FIGURE 1.8: GRAFTING OF A GENERIC SILYL-DERIVATIVE RSI(OR') ${ }_{3}$ ONTO A MESOPOROUS SILICA PORE WALLS. ${ }^{49}$

FIGURE 1.9: CO-CONDENSATION BETWEEN TETRAETHYL ORTOSILICATE (TEOS) AND A GENERIC SILYLDERIVATIVE COMPOUND, RSI $\left(\mathrm{OR}^{\prime}\right)_{3}$, FOLLOWED BY CALCINATION/EXTRACTION TO ACHIEVE HYBRID ORGANIC-INORGANIC MESOPOROUS SILICA. ${ }^{49}$.

Figure 1.10 A: GENERAL SYNTHETIC PATHWAY TO PMOS. ${ }^{49}$ B: REPRESENTATIVE BRIDGED SILSESQUIOXANES. ${ }^{52}$

FIGURE 1.11: PROTONATION/DEPROTONATION OF ORTHOSILICIC ACID........................................ 17

FIGURE 1.12: SCHEMATIC REPRESENTATION OF HYDROLYSIS/CONDENSATION REACTIONS OF TETRAMETHYL ORTOSILICATE (TMOS) UNDER ACID AND BASE CATALYSIS.

FIGURE 1.13: BALL AND STICK REPRESENTATION OF TETRAMETHYL ORTOSILICATE (TMOS), TETRAETHYL ORTOSILICATE (TEOS) AND TETRAPROPYL ORTOSILICATE (TPOS).

FIGURE 1.14: FLUORIDE-CATALYZED HYDROLYSIS (A) AND CONDENSATION (B) OF TETRAMETHYL ORTOSILICATE. ADAPTED FROM REF. 62

FIGURE 1.15: SCHEMATIC REPRESENTATION OF THE POSSIBLE INTERACTIONS BETWEEN SILICA PRECURSORS AND SURFACTANT MOLECULES. ADAPTED FROM REF. 49

FIGURE 1.16 A: BALL AND STICK REPRESENTATIONS OF TWO OF THE MOST COMMON HPA VARIANTS. P (BLACK), Mo (DARK GREY), O (LIGHT GREY). AdAPTED FROM REF. 66 B: MOdEL REACTION FOR SYNTHESIS OF $N, N^{\prime}$-(3-NITROOPHENYLMETHYLENE)DIBENZAMIDE. ${ }^{71}$

FIGURE 1.17: SCHEMATIC REPRESENTATION OF SOME OF THE DIFFERENT CHEMICAL MAKEUPS OF SULFONIC ACID GROUPS. ADAPTED FROM REF. 75

FIGURE 1.18 A: SCHEMATIC REPRESENTATION OF THE GRAFTED AMINES REPORTED IN REF. ${ }^{77}$ B: SYNTHESIS OF GUANIDINE-BASED SILYL-DERIVATIVE. ${ }^{80} \mathrm{C}$ : IMIDAZOLIUM-BASED SILYL-DERIVATIVES. ${ }^{82}$ D: SILICA- 
SUPPORTED PYRROLIDINE-BASED CHIRAL IONIC LIQUID. ${ }^{84} \mathrm{E}: 1,8-B$-BS(DIMETHYLAMINO)NAPHTHALENE (DMAN)-BASED SILYL-DERIVATIVE. ${ }^{83} \mathrm{~F}$ : SILICA-FUNCTIONALIZED PYRROLIDINE-BASED CHIRAL HYBRID CATALYST. ${ }^{85}$

FIGURE 1.19: SCHEMATIC REPRESENTATION OF THE PREPARATION OF MCM-41 SUPPORTED PD COMPLEX. ${ }^{87}$

FIGURE 1.20: SCHEMATIC REPRESENTATION OF THE SYNTHESIS OF SILICA-SUPPORTED CHIRAL RU COMPLEXES. ADAPTED FROM REF. 88

FIGURE 1.21 A: SCHEMATIC REPRESENTATION OF THE SYNTHESIS OF PMOS CONTAINING VANADYL SCHIFF BASE COMPLEXES. ADAPTED FROM REF. 89. B: SCHEMATIC REPRESENTATION OF THE SYNTHESIS OF GOLD NPS IMBEDDED INTO SILICA. ${ }^{90}$

FIGURE 1.22 A: ONE-POT SYNTHESIS OF BI-FUNCTIONAL MESOPOROUS HYBRIDS. B: DMAN GRAFTING ON MESOPOROUS SILICAS CONTAINING SULFONIC ACIDS. ADAPTED FROM REF. 94

FIGURE 1.23: SCHEMATIC REPRESENTATION OF THE SYNTHESIS OF BI-FUNCTIONAL HYBRID CATALYST BARING AMINO FUNCTIONALITIES AND PD-DIAMINO COMPLEXES. INSET: SONOGASHIRA-HENRY TANDEM REACTION USED FOR TESTING THE ACTIVITY OF THE HYBRID CATALYST. ADAPTED FROM REF. $95 \ldots . . . . .37$

FIGURE 1.24 A: SYNTHESIS OF THE BI-FUNCTIONAL HYBRID CATALYST. B: SCHEMATIC REPRESENTATION OF A GENERAL DYNAMIC KINETIC RESOLUTION (DKR) OF A PRIMARY AMINE. C: DKR OF AN AMINE WITH A BI-FUNCTIONAL BIOMIMETIC CATALYST IN WHICH PD NANOPARTICLES AND A LIPASE (CALB) ARE COIMMOBILIZED IN THE SAME PORE OF MCF. ${ }^{96}$

FIGURE 1.25 A: SYNTHETIC PROCEDURE IMPLEMENTED FOR THE SYNTHESIS OF THE PILLARED HYBRID ZEOLITIC MATERIALS FROM MWW PRECURSORS. B: LAYERED HYBRID MATERIAL OBTAINED BY PILLARING WITH BTEB SILSESQUIOXANE MOLECULES (MWW-BTEB). ${ }^{103}$

FIGURE 3.1: SCHEMATIC REPRESENTATION OF THE STRUCTURE OF NAFION POLYMERS ........................ 57

FIGURE 3.2 A: FUNCTIONALIZATION OF CMK-3 WITH FUMING $\mathrm{H}_{2} \mathrm{SO}_{4} .{ }^{28} \mathrm{~B}$ : SULFONATION OF CMK-5 WITH BENZENE PARA-DIAZONIUM SULFONIC ACID. ${ }^{29} \mathrm{C}$ : SYNTHESIS OF SIC-DERIVED SO ${ }_{3} \mathrm{H}$-FUNCTIONALIZED MESOPOROUS CARBON BY HARD-TEMPLATING APPROACH. ${ }^{26}$ D: SYNTHESIS OF $\mathrm{SO}_{3} \mathrm{H}$-FUNCTIONALIZED MESOPOROUS CARBON BY SOFT-TEMPLATING APPROACH. ${ }^{27}$

FIGURE 3.3 A: SYNTHESIS OF $\mathrm{SO}_{3} \mathrm{H}$-FUNCTIONALIZED ORDERED MESOPOROUS POLYMER THROUGH POLY(DIVINYLBENZENE)(POLY-DVB) BY USING TRIMETHOXY SILANE (TMS)-COATED KIT-6 AS TEMPLATING AGENT. TEM IMAGES OF TMS-COATED KIT-6 (B) AND POLY-DVB MATERIALS(C). AIBN=AZOBISISOBUTYRONITRILE. ${ }^{34}$

FIGURE 3.4: LIST OF SOME OF THE PRECURSORS IMPLEMENTED IN THE SYNTHESIS OF SILICA SUPPORTED SULFONIC ACIDS. SAP: SULFONIC ACID PRECURSORS; SAMS: SULFONIC ACID BASED MESOSTRUCTURES; PTS: PHENYLTRIALKOXYSILANE; SALS: SULFONIC ACID LIGANDS; MCS: MESOPOROUS CARBONS. ADAPTED FROM REF. 37.

FIGURE 3.5: SYNTHESIS OF DOUBLE-SULFONATED PHENYLENE-BRIDGED PMO-SO ${ }_{3} \mathrm{H} .{ }^{63} \ldots \ldots \ldots \ldots \ldots \ldots .64$

FIGURE 3.6: COMPARING THE CATALYTIC ACTIVITIES OF FOUR DIFFERENT TYPES OF SULFONATED MESOPOROUS MATERIALS. ADAPTED FROM REF. 65 66

FIGURE 3.7: SYNTHESIS OF A BI-FUNCTIONALIZED PMO FOR NITROALDOL CATALYTIC REACTIONS. ${ }^{66} \ldots \ldots . . .67$

FIGURE 3.8: SCHEMATIC REPRESENTATION OF THE SYNTHESIS OF THE SILICA-SUPPORTED SULFONIC ACIDS $\mathrm{HSO}_{3}-\mathrm{NOS}-\mathrm{H}(\mathrm{A})$ AND $\mathrm{HSO}_{3}-\mathrm{NOS}-\mathrm{F}(\mathrm{B})$

FIGURE 3.9: ${ }^{1} \mathrm{H}(\mathrm{A})$ AND ${ }^{13} \mathrm{C}(\mathrm{B})$ LIQUID NMR SPECTRA OF COMPOUND 2 
FigURE 3.10: TGA AND DTA CURVES OF $\mathrm{HSO}_{3}-\mathrm{NOS}-\mathrm{H}$ (A AND A') AND HSO $\mathrm{HSO}_{3}-\mathrm{NOS}-\mathrm{F}$ (B AND B') ....... 76

FIGURE 3.11 A: $\mathrm{N}_{2}$ ADSORPTION/DESORPTION ISOTHERMS AT $77 \mathrm{~K}$ OF HSO $\mathrm{H}_{3}-\mathrm{NOS}-\mathrm{H}, \mathrm{HSO}_{3}-\mathrm{NOS}-\mathrm{F}$ HYBRID MATERIALS AND NON-ORDERED SILICA (NOS, REFERENCE MATERIAL). B: PORE-SIZE DISTRIBUTION OF THE MATERIALS IN THE MESOPORE RANGE.

FIGURE 3.12: FTIR SPECTRA OF NON-ORDERED SILICA (NOS, CURVE A), $\mathrm{HSO}_{3}$-NOS-F (CURVE B) AND $\mathrm{HSO}_{3}$-NOS-H (CURVE C). SAMPLES OUTGASSED AT $453 \mathrm{~K}$ BEFORE RECORDING THE SPECTRA. ....... 79

FIGURE 3.13 A: SCHEMATIC REPRESENTATION OF Q AND T SILICON SITES DETECTED IN ${ }^{29}$ SI NMR. B: ASSIGNATION OF ${ }^{13} \mathrm{C}$ NMR SIGNALS OF THE HYBRIDS. C: ${ }^{29} \mathrm{SI} \mathrm{CP} / \mathrm{MAS}$ NMR SPECTRA OF THE

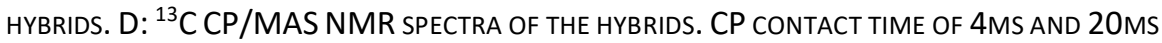
WERE USED FOR SAMPLES $\mathrm{HSO}_{3}$-NOS-H (RED CURVES) AND HSO $\mathrm{H}_{3}$-NOS-F (BLACK CURVES), RESPECTIVELY. SIGNALS RELATED TO UNCONDENSED ALKOXY GROUPS ARE MARKED WITH *.

FIGURE 3.14: ${ }^{1} \mathrm{H}$ MAS (LEFT PANEL) AND ${ }^{1} \mathrm{H}$ SPIN-ECHO (RIGHT PANEL) NMR SPECTRA THE HYBRID CATALYSTS. ABOVE THE SPECTA ASSIGNATION OF THE ${ }^{1} \mathrm{H}$ SIGNALS IS REPORTED. AN ECHO DELAY (TAU) OF 2 MS WAS USED IN THE SPIN-ECHO EXPERIMENT. INSET SHOWS THE ZOOMED SPECTRA.

FIGURE 3.15: FTIR DIFFERENCE SPECTRA IN THE OH (LEFT PANEL) AND CO (RIGHT PANEL) STRETCHING REGION OF CO ADSORPTION AT $80 \mathrm{~K}^{\circ} \mathrm{HSO}_{3}$-NOS-H AND HSO $\mathrm{H}_{3}$-NOS-F HYBRID MATERIALS. ... 83

FIGURE 3.16: FTIR SPECTRA OF $\mathrm{NH}_{3}$ ADSORPTION AT $298 \mathrm{~K}$ ON NOS (A), $\mathrm{HSO}_{3}-\mathrm{NOS}-\mathrm{H}$ (B), $\mathrm{HSO}_{3}$-NOS$\mathrm{F}(\mathrm{C})$ HYBRID MATERIALS. RED CURVES: HYBRIDS BEFORE TO NH $\mathrm{NH}_{3}$ ADSORPTION. BLACK CURVES: 30 MBAR $\mathrm{NH}_{3}$. BLUE CURVES: $\mathrm{NH}_{3}$ OUTGASSING AT RT. B AND C INSETS: FTIR DIFFERENCE SPECTRA. .. 85

FIGURE 3.17: ${ }^{1} \mathrm{H}$ SPIN-ECHO NMR SPECTRA OF THE HYBRID CATALYSTS, BEFORE (BLACK) AND AFTER (RED) AMMONIA ADSORPTION.

FIGURE 3.18: SCHEMATIC REPRESENTATION OF THE INTERACTION BETWEEN THE SILICA SURFACE AND $\mathrm{NH}_{2}$-PR GROUPS $^{87}(A)$, SULFONIC ACID GROUPS IN $\mathrm{HSO}_{3}$-NOS-H MATERIAL IN PRESENCE OF CO (B) AND AMMONIA (C)

FIGURE 3.19 A: ${ }^{31}$ P MAS NMR SPECTRA OF THE HYBRID CATALYSTS RECORDED AT ROOM TEMPERATURE. * REFERS TO SPINNING SIDEBANDS. B: ACID STRENGTH VERSUS ${ }^{31} \mathrm{P}$ MAS NMR CHEMICAL SHIFTS OF VARIOUS SUPER ACIDS CATALYSTS REPORTED IN THE LITERATURE (REF. 93).

FIGURE 3.20 A: SCHEMATIC REPRESENTATION OF THE ACETAL FORMATION REACTION BETWEEN BENZALDEHYDE AND ETHYLENE GLYCOL. B: YIELDS OBTAINED USING CATALYSTS $\mathrm{HSO}_{3}-\mathrm{NOS}-\mathrm{H}$ AND $\mathrm{HSO}_{3}$-NOS-F.

FIGURE 3.21: SCHEMATIC REPRESENTATION OF THE SYNTHESIS OF HYDROPHOBIC SILICA-SUPPORTED SULFONIC ACID CATALYSTS.

FIGURE 3.22 A: SCHEMATIC REPRESENTATION OF THE ACETAL FORMATION REACTION BETWEEN BENZALDEHYDE AND ETHYLENE GLYCOL. B: YIELDS OBTAINED FOR ACETALIZATION REACTIONS USING $\mathrm{HSO}_{3}-\mathrm{CH}_{3} \mathrm{NOS}-\mathrm{H}$ AND HSO $3-\mathrm{CH}_{3} \mathrm{NOS}-\mathrm{F}$ CATALYSTS.

FIGURE 3.23 A: $\mathrm{N}_{2}$ ADSORPTION/DESORPTION ISOTHERMS AT $77 \mathrm{~K}$ OF HYBRIDS HSO ${ }_{3}-\mathrm{NOS}-\mathrm{H}, \mathrm{HSO}_{3}$ NOS-F, $\mathrm{HSO}_{3}-\mathrm{CH}_{3} \mathrm{NOS}-\mathrm{H}$ AND HSO $3-\mathrm{CH}_{3} \mathrm{NOS}-\mathrm{F}$. B: PORE-SIZE DISTRIBUTION OF THE MATERIALS IN THE MESOPORE RANGE

FIGURE 3.24: ${ }^{29} \mathrm{SI}$ CP/MAS NMR SPECTRA OF THE HYDROPHOBIC HYBRID MATERIALS.

FIGURE 3.25: ${ }^{1} \mathrm{H}$ SPIN-ECHO NMR (A) AND ${ }^{13} \mathrm{C}$ CPMAS NMR (B) SPECTRA OF THE HYDROPHOBIC HYBRID MATERIALS. 


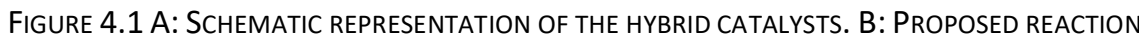
MECHANISMS FOR THE ACID-BASE CATALYZED 4-NITROBENZALDEHYDE WITH ACETONE (REPORTED IN THE INSET). ADAPTED FROM REF. 14.

FIGURE 4.2: HYBRID BI-FUNCTIONAL CATALYSTS TESTED IN THE REACTION OF 4-NITROBENZALDEHYDE WITH ACETONE. ADAPTED FROM REF. 17.

FIGURE 4.3 A: SILICA-SUPPORTED ALKYLAMINES USED AS ADSORBENT AND AS HETEROGENEOUS CATALYSTS. B: AMINE EFFICIENCIES FOR $\mathrm{CO}_{2}$ ADSORPTION (400 PPM IN HE). C: SCHEMATIC REPRESENTATION OF

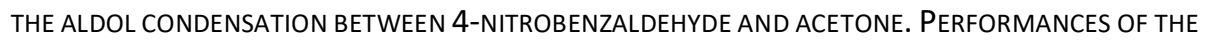
NON-PASSIVATED (D) AND PASSIVATED (E) HYBRIDS IN THE REACTION BETWEEN OF 4NITROBENZALDEHYDE AND ACETONE. AdAPTED FROM REF. 12.

FIGURE 4.4 A: SCHEMATIC REPRESENTATION OF THE CURVATURE EFFECTS ON CONTROLLING THE DISTANCE BETWEEN AMINE GROUPS AND SURFACE SILANOLS. B: INITIAL TURNOVER FREQUENCY OF 4NITROBENZALDEHYDE IN THE ALDOL CONDENSATION WITH ACETONE AT $50^{\circ} \mathrm{C}$. ADAPTED FROM REF. 16.

FIGURE 4.5: ROADMAP OF THE SYNTHESIS OF THE MULTI-FUNCTIONAL HYBRID CATALYST. 117

FigURE 4.6 A: SCHEME OF 3-TRIETHOXYSILYLPROPYLAMINE (APTES, I) AND N-(2-AMINOETHYL)-3(TRIMETHOXYSILYL)PROPYLAMINE (II); SYNTHESIS OF SILYL-DERIVATIVES III, IV, V AND VI. B: SYNTHETIC STEPS IMPLEMENTED IN THE SYNTHESIS OF SILICA-SUPPORTED BASE CATAYSTS 1-6.।): MCM-41, DRY TOLUENE, $1 \mathrm{H}, \mathrm{RT}$; ॥): COMPOUND I, II OR III, 24 H, REFLUX; II) MCM-41, SILYLATED BASE PRECURSOR IV, V OR VI, DRY $\mathrm{CH}_{3} \mathrm{CN}, 1 \mathrm{H}, \mathrm{RT}$, IV) TEA, REFLUX, $24 \mathrm{H}$; V) TMAOH, $\mathrm{CH}_{3} \mathrm{OH}, 30$ MIN.

FIGURE 4.7: ${ }^{1} \mathrm{H}$ NMR SPECTRA OF SILYL DERIVATIVES III, IV, V, AND VI......

FIGURE 4.8: POWDER XRD PATTERNS OF THE MCM-41 SUPPORT AND THE SILICA-SUPPORTED BASE CATALYSTS 1-6.

FIGURE 4.9: TGA AND DTA CURVES OF THE HYBRID BASE CATALYSTS 1-6.

FIGURE $4.10:{ }^{13} \mathrm{C}$ CP/MAS NMR SPECTRA OF THE HYBRID BASE CATALYSTS $1-6$ AND ${ }^{13} \mathrm{C}$ LIQUID NMR SPECTRA OF SILYL-DERIVATIVES I-VI. *SOLVENT RESIDUAL PEAK.

FIGURE 4.11: ${ }^{29}$ SI BD/MAS NMR SPECTRA OF HYBRID BASE CATALYSTS 1-6 AND ${ }^{29}$ SI LIQUID NMR SPECTRA OF SILYL-DERIVATIVES I, II, V AND VI.

FIGURE 4.12: FTIR SPECTRA OF HYBRID MATERIALS 1-6 AFTER OUTGASSING AT $120^{\circ} \mathrm{C} . \ldots \ldots \ldots \ldots \ldots . . . . . . . . .130$

FIGURE 4.13 A: $\mathrm{N}_{2}$ ADSORPTION ISOTHERMS OF THE HYBRID CATALYSTS 1-6. B: BJH PORE SIZE DISTRIBUTION OF CATALYSTS 1-6.

FIGURE 4.14: SCHEMATIC REPRESENTATION OF THE KNOEVENAGEL REACTION OF BENZALDEHYDE AND ETHYL CYANOACETATE, ETHYL ACETOACETATE AND DIETHYL MALONATE.

FiguRE 4.15: CATALYTIC ACTIVITY OF THE HYBRID BASE CATALYSTS (10\% MOL) IN THE KNOEVENAGEL CONDENSATION BETWEEN BENZALDEHYDE AND ETHYL CYANOACETATE.

FiguRE 4.16: CATALYTIC ACTIVITY OF THE HYBRID BASE CATALYSTS 1-3 (10 MOL \% CATALYST LOADING) IN THE KNOEVENAGEL CONDENSATION OF BENZALDEHYDE WITH ETHYL ACETOACETATE (A) AND DIETHYL MALONATE (B).

FIGURE 4.17: PROPOSED CATALYTIC CYCLE OF THE KNOEVENAGEL REACTION OF BENZALDEHYDE AND ETHYL CYANOACETATE AND ADDITIONAL REACTIONS THAT CAN OCCUR. 
FigURE 4.18: CATALYTIC PERFORMANCES OF THE HYBRID CATALYSTS IN THE KNOEVENAGEL CONDENSATION OF BENZALDEHYDE WITH ETHYL CYANOACETATE (2 MOL \% CATALYST LOADING).

FIGURE 4.19: RECYCLING TESTS OF KNOEVENAGEL CONDENSATION BETWEEN BENZALDEHYDE AND ETHYL CYANOACETATE ( 2 MOL \% CATALYST LOADING). REACTION TIME: $4 \mathrm{H}$.

FIGURE 4.20: XRD PATTERNS OF HYBRID BASE MATERIALS BEFORE (SOLID LINE) AND AFTER (DASH LINE) FOUR

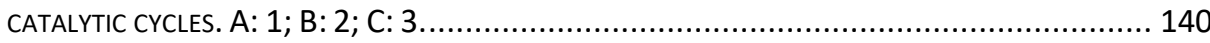

FigURE 4.21: MiCHAEL ADDITION BETWEEN ETHYL 2-OXOCYCLOPENTANE 2-CARBOXYLATE AND METHYL VINYL KETONE.

Figure 4.22: Proposed enolate CATAlytic CyCle of Michael addition betWeEn ethyl 2OXOCYCLOPENTANE 2-CARBOXYLATE (ETOCC) AND METHYL VINYL KETONE (MVK)

FIGURE 4.23: SCHEMATIC REPRESENTATION OF THE ALDOL CONDENSATION BETWEEN FURFURAL (FAL) AND METHYL ISOBUTYL KETONE (MIBK) TO GIVE PRODUCT E.

FIGURE 4.24: PROPOSED MECHANISM FOR THE ALDOL CONDENSATION BETWEEN FURFURAL AND METHYL ISOBUTYL KETONE.

FIGURE 4.25: SCHEMATIC REPRESENTATION OF SOME OF THE MOST COMMONLY USED PHOSPHAZENE BASES. ADAPTED FROM REF. 46. 146

FIGURE 4.26 A: SCHEMATIC REPRESENTATION OF THE SYNTHESIS OF P1-MCM-41. B: ${ }^{13}$ C LIQUID NMR SPECTRUM OF SILYL-DERIVATIVE 3P. C: ${ }^{31}$ P LIQUID NMR SPECTRUM OF 3P IN TOLUENE-D6 (A) AND $\mathrm{D}_{2} \mathrm{O}(\mathrm{B})$

FIGURE 4.27 A: XRD PATTERN OF P1-MCM-41. B: N2 PHYSISORPTION ISOTHERM (77 K) RECORDED ON P1-MCM-41. INSET: BJH PORE SIZE DISTRIBUTION OF P1-MCM-41. C: ${ }^{31} \mathrm{P}$ SOLID STATE MAS NMR SPECTRUM OF P1-MCM-41. D: ${ }^{29}$ SI SOLID STATE CP/MAS NMR SPECTRUM OF P1-MCM41. E: ${ }^{13} \mathrm{C}$ SOLID STATE CP/MAS NMR SPECTRUM OF P1-MCM-41.

FIGURE 5.1: GEOMETRIC AND ELECTRONIC STRUCTURES OF SINGLE ATOMS, CLUSTERS, AND NANOPARTICLES. ${ }^{2}$

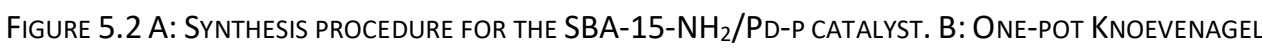
CONDENSATION/HYDROGENATION MULTI-STEP CASCADE REACTION SEQUENCE BETWEEN BeNZALDEHYDE AND ETHYL CYANOACETATE. C: TEM IMAge OF THE SBA-15-NH $\mathrm{N}_{2} /$ Pd-P CATALYST AFTER FIVE CATALYTIC CYCLES. ADAPTED FROM REF. 16.

Figure 5.3 A: TEM IMAgeS OF MCF-SUPPORTED PD NPS. ${ }^{18}$ B: SCHEMATIC REPRESENTATION OF THE SYNTHESIS OF SILICA-SUPPORTED PD NPS. ${ }^{19} \mathrm{C}$ : SCHEMATIC REPRESENTATION OF THE SYNTHESIS OF PD/SBA-16. ${ }^{20}$

FIGURE 5.4: Singlet (A) AND TRIPLET (B) CARBENES. STABILIZATION OF THE SINGLET GROUND STATE BY INTERACTION OF THE P $\pi$ ORBITAL WITH A $\pi$-ELECTRON DONATING X GROUP (C). AdAPTED FROM REF. 27.

FIGURE 5.5: NHCS STRUCTURE AND MESOMERIC STABILIZATION OF THE SINGLET GROUND STATE. ADAPTED FROM REF. 27. 166

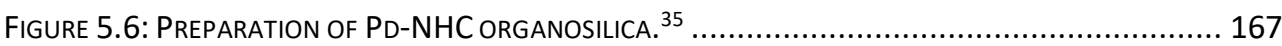

FIGURE 5.7: STRUCTURE OF 2,2'-BIS(DIPHENYLPHOSPHINO)-1,1'-BINAPHTHYL (BINAP). .................. 168 FIGURE 5.8: PROCESSING OPTIONS TO CONVERT BIOMASS INTO VALUABLE FUELS AND FUEL ADDITIVES. ADAPTED FROM REF. 45. 
FIGURE 5.9 A: SCHEMATIC REPRESENTATION OF THE SYNTHETIC ROUTE TO OBTAIN JET-FUEL-RANGE BRANCHED ALKANES FROM FURFURAL. AdAPTED FROM REF. 49. B: SCHEMATIC REPRESENTATION OF THE SYNTHESIS OF 1-(FURAN-2-YL)-4-METHYLPENTAN-2-AMINE FROM FURFURAL. ADAPTED FROM REF. 51.

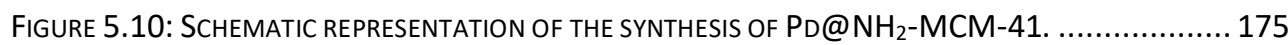

FIGURE 5.11: X-RAY POWDER DIFFRACTION PATTERNS OF CATALYSTS 1 AND 1-PD............................ 176

FIGURE 5.12: TRANSMISSION ELECTRON MICROGRAPHS OF CATALYST 1-PD RECORDED IN BRIGHT-FIELD (A-

B) AND DARK-FIELD (C) MODES. PARTICLE SIZE DISTRIBUTION CALCULATED UPON MEASURING THE SIZE OF 147 PARTICLES OVER A TOTAL OF 8 MICROGRAPHS (D)

FIGURE 5.13: N2 PHYSISORPTION ISOTHERMS AT $77 \mathrm{~K}$ (A) AND BJH PORE SIZE DISTRIBUTION (B) OF PURE SILICA MCM-41, CATALYST 1 AND 1-PD.

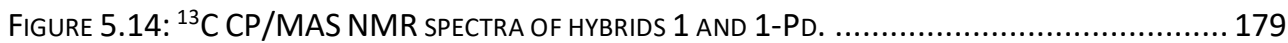

FIGURE 5.15: SCHEMATIC REPRESENTATION OF THE TANDEM REACTION BETWEEN FURFURAL AND METHYL ISOBUTYL KETONE.

FigURE 5.16: REACTION PATHWAYS FOR THE SYNTHESIS OF SILYL-DERIVATIVE PD-BISNHC (A) AND OF

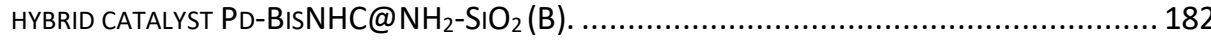

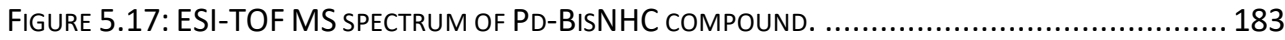

FIGURE 5.18: ${ }^{13} \mathrm{C}$ CP MAS NMR SPECTRUM OF HYBRID PD-BISNHC@ $\mathrm{NH}_{2}-\mathrm{SIO}_{2} \ldots \ldots \ldots \ldots \ldots \ldots \ldots . . . . . . . . . . . . .184$

FIGURE 5.19: 5,5'-DIMETHYLAMINO-2,2'-BIS(DIPHENYLPHOSPHINO)-1,1'-BINAPHTHYL (BAM-BINAP), PROVIDED BY CAGE CHEMICALS (PLATFORM). 3-ISOCYANATOPROPYLTRIETHOXYSILANE (LINKER). MCM-41 (SUPPORT)

FIGURE 5.20: SCHEMATIC REPRESENTATION OF THE SYNTHESIS PATHWAY TO SYNTHESIZE PD-BAM-BINAPMCM-41.

FIGURE 5.21: ${ }^{13}$ C CP MAS NMR SPECTRUM OF PD-BAM-BINAP-MCM-41 MATERIAL. 187

FIGURE 5.22: SCHEMATIC REPRESENTATION OF THE TANDEM REACTION INVOLVING THE SUZUKI COUPLING BETWEEN BENZENEBORONIC ACID AND 4-BROMOBENZALDEHYDE FOLLOWED BY THE ALDOL CONDENSATION OF THE PRODUCT WITH ACETONE. 


\section{List of Tables}

TABLE 3.1: EVALUATION OF THE SCOPE OF THE ANCHORED CATALYST, $\mathrm{HSO}_{3}-\mathrm{NOS}-\mathrm{H}$, FOR ACETALIZATION AND TRANSACETALIZATION REACTIONS.

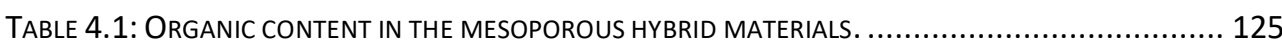

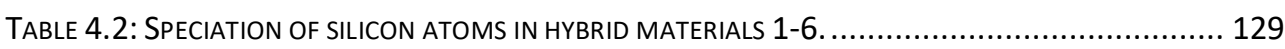

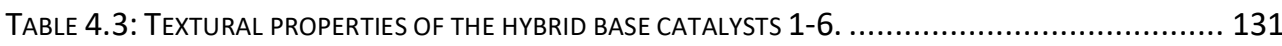

TABLE 4.4: KNOEVENAGEL CONDENSATION BETWEEN BENZALDEHYDE AND ETHYL CYANOACETATE (A), ETHYL

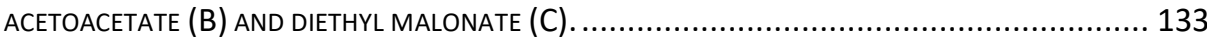

TABLE 4.5: KNOEVENAGEL CONDENSATION OF BENZALDEHYDE AND ETHYL CYANOACETATE. ................. 138

TABLE 4.6: ORGANIC CONTENTS OF HYBRIDS 1-3 BEFORE AND AFTER 4 CATALYTIC CYCLES. .................. 140

TABLE 4.7: LEACHING TESTS OF THE HYBRIDS 1-3 (10\% MOL) IN THE KNOEVENAGEL CONDENSATION OF BENZALDEHYDE WITH ETHYL CYANOACETATE.

TABLE 4.8: MichAEL ADDITION BETWEEN ETHYL 2-OXOCYCLOPENTANE 2-CARBOXYLATE AND METHYL VINYL

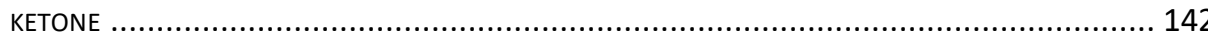

TABLE 4.9: CATALYTIC ACTIVITY OF THE HYBRIDS IN THE CONDENSATION OF FURFURAL AND MIBK ........ 144

TABLE 5.1: COMPOSITIONAL AND TEXTURAL PROPERTIES OF THE HYBRIDS.................................... 179

TABLE 5.2: CATALYTIC ACTIVITY OF 1-PD IN THE TANDEM REACTION BetWEen FF AND MIBK. A ............ 181

TABLE 5.3: CATALYTIC RESULTS IN THE TANDEM REACTION. .................................................. 188 

Scientific Contribution 



\section{$\underline{\text { Peer-reviewed articles }}$}

1. Erigoni, A.; Newland, S. H.; Paul, G.; Marchese, L.; Raja, R.; Gianotti, E.; Creating Accessible Active Sites in Hierarchical MFI Zeolites for Low-Temperature Acid Catalysis, ChemCatChem 2016, 8, $3161-3169$.

2. Erigoni, A.; Paul, G.; Meazza, M.; Hernández-Soto, M. C.; Miletto, I.; Rios, R.; Segarra, C.; Marchese, L.; Raja, R.; Rey, F.; Gianotti, E.; Díaz, U.; Acid properties of organosiliceous hybrid materials based on pendant (fluoro)aryl-sulfonic groups through a spectroscopic study with probe molecules, Catal. Sci. Technol. 2019, 9, 6308-6317.

3. Erigoni, A.; Hernández-Soto, M. C.; Rey, F.; Segarra, C.; Díaz, U.; Highly active hybrid mesoporous silica-supported base organocatalysts for $\mathrm{C}-\mathrm{C}$ bond formation, Catal. Today 2020, 345, 227-236.

4. Erigoni, A.; Díaz U.; Porous silica-based organic-inorganic hybrid catalysts: a review, submitted. 


\section{Contributions at conferences and congresses}

Title: Hybrid Super-Acid Solid Catalysts for Anisole Friedel-Crafts Acylation.

Authors: A. Erigoni, U. Díaz, M. C. Hernández-Soto, C. Segarra, F. Rey.

Conference: NanoInnovation2017 (Rome IT, 2017).

Title: Organosiliceous Hybrid Base Catalysts - Synthesis and Reactivity.

Authors: A. Erigoni, U. Díaz, C. Segarra, M. Hernández, F. Rey.

Conference: EFCATS school (Liblice CZ, 2018).

Title: Hybrid Acid Catalysts: Synthesis and Characterization.

Authors: A. Erigoni, C. Segarra, I. Miletto, G. Paul, F. Rey, E. Gianotti, L. Marchese, U. Diaz.

Conference: 6th International Conference on Multifunctional, Hybrid and Nanomaterials (Sitges ES, 2018).

Title: Hybrid Mesoporous Silica-Supported Base Organocatalysts for C-C Bond Formation.

Authors: A. Erigoni, M.C. Hernández-Soto, F. Rey, C. Segarra, U. Diaz.

Conference: XVI Simposio de Jóvenes Investigadores de la Real Sociedad Española de Química (Valencia, 2019).

Title: Hybrid Acid Catalysts: Synthesis and Spectroscopic Characterization.

Authors: A. Erigoni, I. Miletto, G. Paul, F. Rey, E. Gianotti, L. Marchese, U. Diaz.

Conference: AIZ 2019 congress joint with the 8th CIS conference (Amantea IT, 2019). 


\section{Permanence at international research institutes}

Eastern Piedmont University (Alessandria, IT)

2018 (3 weeks)

Characterization of hybrid catalysts featuring supported aryl-sulfonic acid groups through multiple spectroscopic techniques. Adsorption of various probe molecules followed by NMR and FTIR spectroscopies.

Supervisor: Prof. Enrica Gianotti

Massachusetts Institute of Technology (Cambridge MA, USA)

2018-2019 (6 months)

Hydrothermal continuous flow synthesis of zeolites and zeotypes using a custom designed flow reactor

Supervisor: Prof. Yuriy Roman-Leshkov 